\title{
Hydroxyl-apatite coated hip implants
}

Citation for published version (APA):

Geesink, R. G. T. (1988). Hydroxyl-apatite coated hip implants. [Doctoral Thesis, Maastricht University]. Rijksuniversiteit Limburg. https://doi.org/10.26481/dis.19880603rg

Document status and date:

Published: 01/01/1988

DOI:

10.26481/dis.19880603rg

Document Version:

Publisher's PDF, also known as Version of record

\section{Please check the document version of this publication:}

- A submitted manuscript is the version of the article upon submission and before peer-review. There can be important differences between the submitted version and the official published version of record.

People interested in the research are advised to contact the author for the final version of the publication, or visit the DOI to the publisher's website.

- The final author version and the galley proof are versions of the publication after peer review.

- The final published version features the final layout of the paper including the volume, issue and page numbers.

Link to publication

\footnotetext{
General rights rights.

- You may freely distribute the URL identifying the publication in the public portal. please follow below link for the End User Agreement:

www.umlib.nl/taverne-license

Take down policy

If you believe that this document breaches copyright please contact us at:

repository@maastrichtuniversity.nl

providing details and we will investigate your claim.
}

Copyright and moral rights for the publications made accessible in the public portal are retained by the authors and/or other copyright owners and it is a condition of accessing publications that users recognise and abide by the legal requirements associated with these

- Users may download and print one copy of any publication from the public portal for the purpose of private study or research.

- You may not further distribute the material or use it for any profit-making activity or commercial gain

If the publication is distributed under the terms of Article $25 \mathrm{fa}$ of the Dutch Copyright Act, indicated by the "Taverne" license above, 


\section{Hydroxyl-Apatite Coated Hip Implants}

\section{Proefschrift}

ter verkrijging van de graad van doctor aan de Rijksuniversiteit Limburg te Maastricht, op gezag van de Rector Magnificus, Prof. Dr. F.I.M. Bonke, volgens het besluit van het College van Dekanen, in het openbaar te verdedigen op vrijdag, 3 juni 1988 om 16.00 uur

door

Rudolph Gerbrand Theodoor Geesink geboren te Arnhem 
Promotores

prof. dr. A.J. van der Linden

prof. dr. K. de Groot, Amsterdam

Beoordelingscommissie prof. dr. G. Kootstra

prof. dr. ir. R. Huiskes, Nijmegen

prof. dr. H.K.L. Nielsen, Groningen

prof. dr. F. Bosman

prof. dr. S. van der Linden 
opgedragen aan allen die mij dierbaar zijn 
Part of this work was awarded the

"1987 Sir John Charnley Award"

Hip Society, San Francisco, January 21th 1987.

Part of this work was published in the following journals:

Geesink RGT, Groot K de, CPAT Klein: Bone bonding to apatite coated implants.

Journal of Bone and Joint Surgery 70B: 17-22, 1988.

Geesink RGT, Groot K de, CPAT Klein: Chemical implant fixation using hydroxyl-apatite coatings. Clinical Orthopaedics and Related Research 225: 147-170, 1987.

Groot K de, Gecsink RGT, CPAT Klein, Serekian P: Plasma-sprayed coatings of hydroxyl-apatite. Journal of Biomedical Materials Research, 21: 1375-1381, 1987. 


\section{Table of contents}

Introduction

Principles of cementless implant fixation (10)

Implant materials (13)

Characteristics of bone and calcium-phosphate ceramics

Characteristics of bone (17)

Calcium-phosphate ceramics

Plasma-spraying and hydroxyl-apatite coatings

Technique of plasma-spraying (27)

Physico-chemical analysis of hydroxyl-apatite coatings

Methods of testing (33)

Physical testing results (36)

Discussion (37)

Cadaver implantation and retrieval of hydroxyl-apatite coated implants 39

Plug implant study

Materials and methods (43)

Results (45)

Discussion

Canine hydroxyl-apatite-coated total hip replacement

Materials and methods (60)

Results (67)

Radiographic evaluation of prostheses. (67)

Scintigraphic evaluation of prostheses (76)

Mechanical evaluation of prostheses. (78)

Histological evaluation of prostheses. (80)

Histology of non-coated titanium prostheses (81)

Histology of hydroxyl-apatite coated prostheses

Discussion (98) 
Infection in canine hydroxyl-apatite coated total hip arthroplasty

Human total hip replacement using hydroxyl-apatite coatings

Implant materials (110)

Prosthesis design (110)

Surgical factors (111)

Clinical use of apatite coated hip prostheses (113)

Grading of clinical results (117)

Clinical results (121)

Radiological findings (124)

Technetium and indium scanning (126)

Discussion (132)

General conclusions and summary

Samenvatting

Resume

145

Literature references

149

Clinical data forms

161

Acknowledgements 


\section{Chapter one}

\section{Introduction}

Total hip replacement is the treatment of choice for end stage destructive hip disease. This operation is performed frequently and its rate is increasing. In the Netherlands over 12.000 total hip replacements are performed each year. Estimations are that world-wide over 2.5 million people live with artificial hip joints. Important indications for this operation include advanced stages of osteo-arthrosis, reumatoid arthritis, avascular necrosis of the femoral head and traumatic affections of the hip joint.

Artificial joint replacement serves the dual purpose of both restoration of natural joint motion and the transfer of joint forces to the bone. The essential problem of artificial joint replacement is at the interface of the inert implant to the living bone. At the same time, the prosthesis should perform well as long as possible and withstand loading forces of up to four times body-weight during daily activities (Crowninshield 1978). Implant design and the method of implant fixation to the bone, in conjunction with the biological response of the patient, are probably the single most critical determining factors for both initiation and propagation of implant-bone interface failure (Huiskes 1986).

Current methods of implant fixation rely on mechanical fixation either with or without the use of acrylic bone cement. Acrylic bone cement (polymethyl-methacrylate) provides a convenience in filling the gap between prosthesis and surrounding bone. The interdigitation between bone cement and trabecular bone is responsible for the mechanical fixation. Bone cement is no glue, there are no bonding properties between bone cement and bone.

Cemented total hip replacement has become one of the most successful orthopaedic procedures today, in that it rather consistently results in relief of pain and disability. Short term results of these cemented artificial hip replacements usually are excellent. However, long term results are obscured by an ever increasing rate of implant loosening. Ten year loosening rates after total hip replacement vary widely, depending upon the definition of what effectively constitutes implant loosening (Brand ea. 1986). Revision rates alone do not represent all loose implants, many older patients are not reoperated for medical or other reasons, although they may have serious complaints. Radiological signs of a loose implant-bone interface are not necessarily accompanied by relevant clinical signs, nor are they absolute indications for revision surgery. Revision rates after ten year follow-up of cemented total hip replacement (Charnley type) are reported ranging between 1.6 percent (Wroblewski 1986, Charnley $1983 \& 1973$ ) and 46 percent (Almby ea. 1982). The radiological incidence of implant loosening is reported to be between 7 percent (Salvati ea. 1981) and 93 percent (Chandler ea. 1981) of cases after a ten year follow-up period of the same (Charnley) prosthesis. Comparable rates of implant loosening are reported for related types of cemented hip implants (van Rens 1986). Especially in the younger age groups the incidence of implant loosening after cemented total hip replacement is high (Cornell ea. 1986, Halley ea. 1986, Collis 1984, Dorr ea. 1983, Chandler ea. 1981). 
The loosening of cemented hip implants can be ascribed to various factors:

- Mechanical degradation of the acrylic bone cement with time (Gates ea. 1984, Krause ea. 1982, Lange 1979, Halawa ea. 1978).

- Impairment of mechanical strength by entrapment of air, blood and laminations in bone cement (Gruen ea. 1976).

- Adverse tissue response initiated by the thermal-chemical side-effects of the acrylic cement (Eriksson 1984, Mjoberg ea. 1984, Goldring ea. 1983, Pedersen ea. 1983, Linder 1977, Reckling 1977, Linder 1976, Feith 1975).

- Compromise of host tissue immunological response by persistent monomer leakage from the acrylic cement (Gristina ea. 1985 \& 1976, Boyd 1984, Endler ea. 1982, Panush ea. 1978, Petty 1978, Linder 1976, Green 1975).

Many of the earlier results of cemented total hip replacement were obtained using implants and cementing techniques that are no longer considered optimum by current standards. Increased knowledge from the field of orthopaedic biomechanics (Huiskes ea. $1986 \& 1985 \& 1983 \& 1979$, Oh 1983, Crowninshield ea. 1980) has led to improvements in implant design and cementing technique including the use of bone lavage, vacuummixing or centrifuging during preparation of bone cement and femoral plugging with pressurization during injection of bone-cement. These improvements have reduced the rates of aseptic implant loosening considerably, thereby improving longterm clinical results (Mattingly ea. 1985, Harris ea. $1984 \&$ 1982). Nevertheless, the earlier problems associated with cemented implant fixation have aroused a lot of interest into methods of implant fixation avoiding the use of bone cement. Although some clinical results are very encouraging (Engh ea. 1987), we must still realize that the elimination of bone cement does not eliminate the problems of initial implant fixation. The principles involved with cementless implant fixation will be dealt with in the next paragraphs.

\section{Principles of cementless implant fixation}

Cementless fixation of implants has been realized using the principle of "osseointegration." Through the use of chemical non-reactive (called bioinert) materials, such as titanium, a close approximation between implant and bone can be realized (Barth ea. 1985, Branemark ea. 1977). The fixation of such implants must rely on principles of mechanical retention because the implant material lacks any adherence to bone.

Mechanical interlock with the surrounding bone is possible by bony ingrowth into macroporous or micro-porous structures on the implant surface. Adequate pore-size, mechanical stability at the implant-bone interface and intimate implant-bone contact are absolute prerequisites for bony ingrowth (Cook ea. 1985, Ronningen ea. 1985 \& 1984, Anderson 1984, Pilliar ea. 1983, Gitelis ea. 1982, Bobyn ea. 1980, Ducheyne ea. 1978, Cameron 1973).

Geometrical design of the implant is responsible for achieving initial mechanical stability. Because trabecular bone is not able to withstand high loading forces, the prosthesis must rest on the stronger cortical bone. In practice, this means that the prosthetic geometry 
should have a close conformity to the internal anatomy of the proximal femur (Crowninshield ea. 1980). Otherwise high stress concentrations in the bone are likely to occur, with threat of implant loosening. At the same time, the difference in stiffness between implant material and bone may not be too great. Under normal loading conditions of an implant, the distribution of stresses between implant and bone is primarily determined by their relative stiffness. The stiffer of the two materials will take the major part of the stress by preventing deformation of the other component. Implant stiffness is a combined effect of prosthetic cross-sectional geometry and modulus of elasticity of the metal used. An equivalent stiffness between prosthesis and bone does not prevent micromotion under bending forces in a curved structure like a proximal femur.

Micro-motion at the prosthesis-bone interface causes localized bone resorption, counteracting bony ingrowth (Perren 1983). Too high an implant stiffness will take the stress away from the bone by preventing any bone deformation. This phenomenon, called "stress-shielding", causes bone resorption of a more diffuse nature. The optimal anatomic design of prosthetic geometry and the use of titanium with its lower modulus of elasticity, as compared to chrome-cobalt alloys, can provide the possibility of satisfying results. Bony ingrowth in porous-metal coatings is certainly possible and experimental studies report values of implant-bone fixation as high as 17 to $25 \mathrm{MPa}^{1}$ in shear stress (Cook ea. 1985, Anderson 1984, Pilliar ea. 1983, Bobyn ea. 1980).

Although early clinical results of porous metal coated hip implants are promising (Engh ea. 1987, Lord ea. 1983), there remain some practical and theoretical limitations with these kinds of implants. The first concern regards clinical performance. In the first two years after implantation of a cementless hip implant, there is a high incidence of midthigh-pain. Percentages as high as 15-40 percent are known. Although these complaints often disappear between the first and second year, functional recovery of patients in this period is inadequate. The cause of these complaints is not precisely known, but appears to be the micro-motion between implant and bone. Micromotion can be caused by instability of the implant through geometrical mismatch between implant and bone, causing toggle of the stem tip or rotational movements of the implant. Another cause of "mid-thigh pain" is probably grossly abnormal stress-transfer from implant to proximal femur. Both a loose distal stem with movements around the stem tip and rigid distal implant fixation with excessive load transfer near the stem tip give rise to concentration of bone stresses in a small area around the stem tip. This causes local hyper-reactive bone proliferation and thereby possibly mid-thigh pain. Additionally, in the case of rigid distal implant fixation, the proximal femur becomes permanently unloaded and thereby is prone to stressshielding osteoporosis.

The second issue of concern is one of more theoretical consideration. Because there is no adherence of the implant metal to the bone, the actual implant fixation is limited to the area available for the surface pores. Increasing surface porosity might increase bone bonding, but at the same time reduces the fatigue limits of the porous metal (Manley ea. 1987). In practice these phenomena put a limit at a surface porosity of about 30 percent.

$1 \mathrm{Mpa}=1$ megapascal $=9,87 \mathrm{~kg} / \mathrm{cm}^{2}=144 \mathrm{psi}$ 
Theoretically, this limits the highest obtainable interface strength to 30 percent of the strength of cortical bone. The shear strength of cortical bone is reported to be between 60 and $80 \mathrm{MPa}$ (Carter ea. 1978). Therefore a maximum interface shear strength of about 20 $\mathrm{MPa}$ is realistic for porous metal coated devices and conforms to the results, previously referenced from the literature. An interface strength of this magnitude can theoretically provide adequate implant performance. There are however other reasons for concern. Bony ingrowth in surface pores is certainly possible under the favorable conditions of early post-operative hyper-vascularity of the implant bed. The bone within the porous metallic structure is however effectively shielded from stress, this in addition to any possible stressshielding caused by mismatch in modulus of elasticity between metal and bone. Bone resorption by disuse atrophy is thus conceivable and has indeed been predicted for certain conditions from finite-element-method calculations (Huiskes ea. 1987) and observed under experimental (Pilliar ea. 1979) and clinical (Engh ea. 1987) conditions.

Furthermore, the longterm biological effects of increased metal-ion release by large internal porous metal surfaces remain unclear (Ryu ea. 1987, Brown ea. 1985, Gaechter ea. 1977) and "bead disease" is a frequent problem. This radiologically visible loosening of beads from the metal substrate indicates failure of implant fixation (Buchert ea. 1986, Rosenqvist ea. 1986, Cook ea. 1984, Yue ea. 1984) and points to the weak fatigue properties of many porous metal coatings. There is in general, growing evidence that biological implant fixation using porous-metal coatings does not meet the original expectations for bony ingrowth, that the surgical technique is much more critical as compared to cemented hip implant surgery and that there still are many unresolved questions as to the biocompatibility of the materials in use (Haddad ea. 1987, Volz ea. 1986). Additionally, human cadaveric retrievals of clinically well-performing implants have generally failed to meet the expectations for bony ingrowth into these porous metal coatings (Bobyn ea. 1984). In practice, many of these types of implants prove to be fixed in fibrous tissue. Although some authors (Walker ea. 1984) suggest that such a fibrous tissue layer can be effective in distributing interface stresses towards the surrounding bone, we must realize that the mechanical loading limits for fibrous tissue are so low, that they cannot be expected to bear any significant interface stresses in any physiological way. The thickness of fibrous tissue membranes around cementless implants appears progressive with loading and movement of the implant (Hedley ea. 1982), thereby increasing (micro-)motion of the implant in a vicious circle. Therefore, "pressfit" implantation using a soft fibrous tissue interface is prone to failure.

In conclusion, many materials currently in use for both cemented and non-cemented artificial hip replacements have some inherent flaws, caused by their limited biocompatibility characteristics. The need for improved biological implant materials is obvious. 


\section{Implant materials}

For optimal performance, materials in use for artificial implants should meet the following requirements:

Mechanical strength

Biocompatibility

Biostability

There is no accepted standard for classification of implant materials. Implant materials, or biomaterials, can be classified either according to their chemical composition or with respect to their biological response in the body. Currently available implant materials can be classified according to their chemical composition in the following groups:

Chemical classification of biomaterials

Metals

Polymers

Ceramics

Composites

\section{Metals}

Metals, in particular stainless steel, chrome-cobalt- and titanium-alloys, have a long clinical history of application in orthopaedic implants (Semlitsch 1983) and need no special discussion.

\section{Polymers}

Of the polymers, poly-ethylene, either in HDPE (high density poly-ethylene) or UHMWPE (ultra high molecular weight poly-ethylene), is frequently used for orthopaedic implants, mainly for moving parts in contact with metal to improve the friction characteristics.

Examples are acetabular cups or tibial components for artificial knee implants. There is some concern regarding the longterm wear rates of poly-ethylene and consequently the biological effects of poly-ethylene wear particles. Abrasion of poly-ethylene particles causes some granulomatous and inflammatory response in the local tissues and could thereby contribute to some degree to implant loosening, especially when the wear particles can reach the cement-bone interface (Charnley 1983 \& 1975, Austin 1982, Rose ea. 1980 \& 1979, Crugnola ea. 1977). Use of smaller size femoral heads (Charnley's "low friction arthroplasty of the hip" 1983) or use of ceramic femoral heads in total hip implants reduces friction, and thereby wear rates. It is essentially a materials problem and it is expected that improvements in quality of poly-ethylene by further increase of its molecular weight will decrease its wear rates. 


\section{Ceramics}

Ceramic materials are relatively new to orthopaedics and chemically, they are a diverse group. They have in common, that they are being manufactured at very high temperatures (usually over $1000^{\circ} \mathrm{C}$ ). In practice, this means that ceramic materials are relatively stable and inert in the body. We can distinguish the group of metal-oxide ceramics, of which only alumina-oxide ceramic $\left(\mathrm{Al}_{2} \mathrm{O}_{3}\right)$ has gained some clinical use as an implant material (Mittelmeier 1981). Secondly, there is the group of carbon-fiber ceramics in its various forms (Christel ea. 1987). Applications are still experimental, although some of its physical characteristics are appealing. A third important group of ceramics consists of those containing calcium-phosphate. Many representants of this group show bio-active behaviour. They will be dealt with in the next chapter.

\section{Composites}

Composite materials are composed of two or more materials into a new material with enhanced characteristics. The body itself can already be looked to as a source of creative ideas for the design of composites.

Both bone, cartilage, tendon, collagen and other connective tissues have composite structures on many hierarchical levels. Also, in industrial technology composite materials find wide-spread application. The structure and complexity may vary depending on the tissue or material involved, but the basic principle is to combine two or more components so as to program desirable and favorable properties, while at the same time avoiding some of the less desirable characteristics of the individual components. Optimal coherence between the individual components is of crucial importance for their proper functioning. Composite materials offer a rational solution to many biomaterials problems (Katz 1984).

Another classification for biomaterials, which is gaining more and more acceptance (Osborn 1979), is one with respect to the biological response, evoked by the materials in the body. This classifies implant materials in the following groups:

\section{Biological classification of biomaterials}

Bio-tolerant

Bio-inert

Bio-active

\section{Bio-tolerant materials}

Biotolerant materials are accepted by the body to a certain degree, but are encapsulated by a thinner or thicker fibrous tissue membrane. This fibroblastic response is evoked, because these materials are not quite inert to the body, leaking irritative chemical agents to the body surroundings. Examples of bio-tolerant materials are acrylic bone-cement with its persistent leakage of monomer (Boyd 1984, Endler ea. 1982, Petty 1978, Gristina ea. 1976, Linder 1976, Green 1975) and the stainless-steel and chrome-cobalt alloys with their leakage of metal-ions (Buchert ea. 1986, Michel 1984, Kummer ea. 1983, Michel 1980, Galante 1972). Although bone cement is sometimes observed in direct contact with bone at certain areas of its bony interface (Draenert ea. 1978), the occurrence of this phenomenon is too infrequent too alter its biological classification. 


\section{Bio-inert materials}

When the surface of an implant material is chemically completely neutral, without any leakage of foreign material, the material can be classified as bio-inert. Under favorable conditions of mechanical rest bone can grow up to the implant surface with no intervening fibrous tissue layer. An example, with frequent application in orthopaedic surgery, is titanium that has a surface-layer of titanium-oxide. This effectively seals the metal from the bone and gives titanium the surface characteristics of a ceramic material (Anderson 1984, Kummer 1983, Semlitsch 1983), alumina-oxide ceramic is another example. All metal-oxide and carbon-fiber ceramics are classified as bio-inert, this means that they can be very well accepted in close proximity to the bone. Nevertheless, by lack of any adherence to the bone, they must still rely on mechanical retention techniques for purposes of skeletal anchoring.

\section{Bio-active materials}

Implant materials are called bio-active when they are capable of bonding with the surrounding bone. This is caused by the presence of free calcium and phosphate compounds at their surfaces, in contact with the bone. Examples of bio-active implant materials include bioglass, tricalcium-phosphate and hydroxyl-apatite. Bioglass is a composition of silicon-oxide ("glass") with various additions of calcium-oxide $(\mathrm{CaO})$ and phosphorus-pentoxide $\left(\mathrm{P}_{2} \mathrm{O}_{5}\right)$. Under favorable conditions, a surface layer of hydroxylapatite on the bioglass develops after implantation. It is this thin hydroxyl-apatite surface layer, that is responsible for the bone-bonding properties of bioglass. The formation of this hydroxyl-apatite layer on bioglass, and therefore its biological performance, are too variable and too dependant upon local tissue properties, to be reliable for implant fixation (Ducheyne 1984 ea. \& 1979, Scharbach 1983, Fuchs ea. 1981, Gross ea. 1981, Sela 1981, Ogino ea. 1980, Griss ea. $1979 \& 1978$ \& 1977, Hench ea. 1977).

Of the group of bio-active calcium-phosphate ceramics, hydroxyl-apatite has currently the best prospects to serve as a permanent implant material. Tricalcium-phosphate is resorbable and therefore not suitable for use as a permanent implant material. In order to understand the physico-chemical behaviour of hydroxyl-apatite and its inter-relation with bone, some data regarding bone and calcium-phosphate ceramics will be provided in the next chapter. 



\section{Chapter two}

\section{Characteristics of bone and calcium-phosphate ceramics}

To understand the biocompatibility and clinical applications of hydroxyl-apatite the following aspects of bone and calcium-phosphate ceramics need special attention:

- Physico-chemical composition

- Microstructure

- Mechanical properties, including fatigue behaviour

- Biocompatibility

- Biodegradation characteristics

- Clinical experience

\section{Characteristics of bone}

Physico-chemical characteristics of bone.

Bone is a mineralized tissue with a complex composition.

It consists of a fibrous organic matrix which is permeated by inorganic salts. The organic component is mainly collagen (90-95\%), further glycosaminoglycans ( $1 \%)$ and other proteins $(5 \%)$ (Carter ea. 1978). The collagen fibers are arranged in a parallel fashion, each fiber being a specific aggregation of collagen macro-molecules. During bone formation mineral crystals are deposited parallel to the longitudinal axes of the collagen fibers. The mineral component of the bone is constituted by an incompletely understood calciumdeficient hydroxyl-apatite crystal. X-ray diffraction analysis of bone reveals hydroxylapatite as the only apparent component. However, other bone minerals are probably so finely dispersed, they appear amorphous to X-ray diffraction analysis (Driessens 1980). With more refined methods of analysis (Driessens 1980) the probable phase composition of the mineral in bone is as follows:

15 percent magnesium-whitlockite with the approximate formula $\mathrm{Ca} 9 \mathrm{Mg}\left(\mathrm{HPO}_{4}\right)\left(\mathrm{PO}_{4}\right)_{6}$

25 percent sodium and carbonate containing apatite with the approximate formula $\mathrm{Ca}_{8.5} \mathrm{Na}_{1.5}\left[\left(\mathrm{PO}_{4}\right)_{4.5}\left(\mathrm{CO}_{3}\right)_{1.5}\right] \mathrm{CO}_{3}$

60 percent carbonated calcium-phosphate with a structure similar to that of octocalcium-phosphate and the approximate formula $\mathrm{Ca}_{8}\left(\mathrm{PO}_{4}\right)_{4}\left(\mathrm{CO}_{3}\right)(\mathrm{OH})_{2}$.

The predominant constituents of these bone minerals are calcium, phosphate and hydroxyl groups with the simplified formula of

$$
\text { hydroxyl-apatite: } \mathrm{Ca}_{10}\left(\mathrm{PO}_{4}\right)_{6}(\mathrm{OH})_{2} \text {. }
$$

Because of the incomplete crystallization of hydroxyl-apatite its calcium ions are easily exchangeable for others like sodium, magnesium, fluoride or foreign ionized components.

During its maturation, bone becomes increasingly mineralized and this seems to be 
associated with collagen intermolecular cross-linking (Lees ea. 1977). The resulting structure, being a composite, is much stronger and stiffer than its individual constituents. The collagen fibers provide the tensile strength of the bone, whereas the hydroxyl-apatite provides the compressive strength. This unique composition gives bone its specific physical properties. The static physical properties of bone are summarized below for cortical (Carter ea. 1978, Saha 1977, Evans 1973, Currey 1970) and trabecular bone (Albright 1977, Wainwright 1976, Evans 1973, Currey 1970). The figures are rather variable and strongly depend on the direction of measurement in the bone and its degree of mineralization, especially in the case of trabecular bone.

Table I

Bone, physical properties

\begin{tabular}{llrl}
\hline & $\begin{array}{l}\text { cortical bone of } \\
\text { longitudinal } \\
\mathrm{MPa}\end{array}$ & $\begin{array}{l}\text { femur } \\
\text { tangential } \\
\mathrm{MPa}\end{array}$ & $\begin{array}{l}\text { trabecular bone } \\
\mathrm{MPa}\end{array}$ \\
\hline compressive strength & $179-209$ & 150 & $4-70$ \\
shear strength & $43-50$ & - & $0.5-5$ \\
tensile strength & $129-140$ & 13 & - \\
modulus of elasticity & $16-19.000$ & 10.000 & - \\
\hline
\end{tabular}

Bone has an extensive vascular supply to sustain its high metabolic and remodelling activity. This remodelling of bone is not only a way of repairing minor and major damage but also a mechanism to adapt to alterations in stress distribution.

Remodelling phenomena depend on the direction and the amount of stress induced in the bone according to "Wolfs law" of bone architecture (Huiskes ea. 1987 \& 1984, Rubin ea. 1984, Frost 1983, Wolff 1892).

\section{Calcium-phosphate ceramics}

\section{Chemistry of calcium-phosphate ceramics}

Calcium-phosphate ceramics have a complex chemistry. From a practical point of view, the single two most important representatives of this group are hydroxyl-apatite and tricalcium-phosphate (or B-whitlockite). Their physico-chemical and biological characteristics have been described extensively in the literature (Brill ea. 1987, Blitterswijk ea. 1986, Holmes ea. 1986, Patka 1985, Shimazaki ea. 1985, de Groot 1984 \& 1983 \& 1981 \& 1980, Krajewski ea. 1984, Uchida ea. 1984, Klein 1984 \& 1983, Flatley ea. 1983, Driessen 1982, Jarcho 1981 \& 1977, Christoffersen 1981, Winter ea. 1981, de With 1981, Denissen 1980, Harms 1979, Peelen 1978, Rejda 1977, Young 1975, Kay ea. 1964). The essentials are summarized below.

\section{Preparation of calcium-phosphate ceramics}

The preparation of calcium-phosphate ceramics starts with calcium-phosphate powders. An example of how to prepare a ceramic block from such a powder is as follows. Hydroxylapatite powders with the appropriate chemical formula $\mathrm{Ca}_{10}\left(\mathrm{PO}_{4}\right)_{6}(\mathrm{OH})_{2}$ and a $\mathrm{Ca} / \mathrm{P}$ ratio of $10 / 6$ are available commercially. The powder is compressed in a stainless steel die with 
a pressure of up to $80 \mathrm{MPa}$. Stearic acid is added as a lubricant to the die. After compression molding and drying, the powder compact is gradually heated and subsequently sintered at temperatures up to $1250^{\circ} \mathrm{C}$. With apatite starting powders, no phase changes occur in the crystal up to temperatures of $1300^{\circ} \mathrm{C}$. Higher temperatures do result in phase transitions of the hydroxyl-apatite crystal with loss of specific hydroxylapatite characteristics.

The starting powders of other compositions, e.x. tricalcium-phosphate with a formula of $\mathrm{Ca}_{3}\left(\mathrm{PO}_{4}\right)_{2}$ and a $\mathrm{Ca} / \mathrm{P}$ ratio of $3 / 2$, result in ceramics with a different structure. Although starting powders with the composition of $\mathrm{Ca}_{3}\left(\mathrm{PO}_{4}\right)_{2}$ have the same crystal lattice structure as hydroxyl-apatite, they do not have enough calcium ions to fill all apatite lattice sites. The crystal shows many vacancies and during sintering these lattices undergo phase changes at temperatures above $800{ }^{\circ} \mathrm{C}, \mathrm{B}$-whitlockite or tricalcium-phosphate being the end result.

\section{Porosities of calcium-phosphate ceramics}

When the starting powder is mixed with hydrogen-peroxide solution ( 0.3 to $1 \%)$ a macroporous ceramic is obtained with pore diameters between 100-300 microns, allowing bony ingrowth. Otherwise, the resultant ceramic will have a dense structure with only a small percentage (1-5\%) of micropores that have a diameter of $1-5$ microns.

\section{Physico-chemical analysis of calcium-phosphate ceramics}

The starting powders and the sintered materials can be analyzed for their mineral composition and crystallographic structure by X-ray diffraction analysis and infrared light spectroscopy. Each crystal structure has a specific diffraction pattern (Fig. 1). Through the use of these techniques, differentiation between hydroxyl-apatite and tricalciumphosphate ceramics is possible. X-ray diffraction analysis is the more accurate and is therefore most widely used, also in this study.
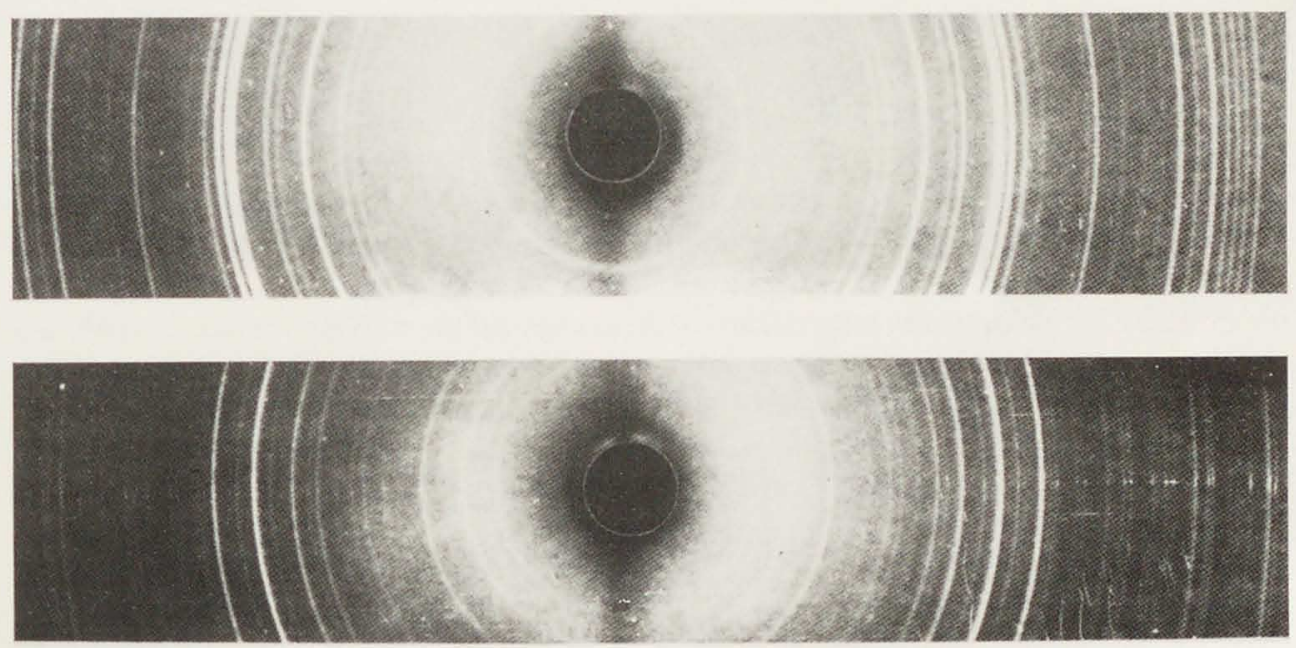

Fig. 1

$\mathrm{X}$-ray diffraction patterns of hydroxyl-apatite (upper) and tricalcium-phosphate (lower). 


\section{Mechanical properties of calcium-phosphate ceramics}

Dense calcium-phosphate ceramics of both hydroxyl-apatite and tricalcium-phosphate lattice structure have a compressive strength up to $500 \mathrm{MPa}$ (de Groot 1983). This corresponds favorably with the compressive strength of cortical bone, reported to be between 179-209 $\mathrm{MPa}$ (Albright 1977), and even with the strongest biological material i.e. dental enamel that has a compressive strength of $500 \mathrm{MPa}$.

Tensile strength of $100 \%$ dense hydroxyl-apatite is approximately $200 \mathrm{MPa}$. Macropores and micropores cause a considerable weakening of its mechanical characteristics. Even a small reduction in density from $100 \%$ to $95 \%$ reduces the tensile strength to approximately $50 \%$. Porous ceramics are therefore not very well suited for implants subject to heavy mechanical loading.

Unfortunately, some biomechanical properties of sintered hydroxyl-apatite are very poor. Although the static mechanical properties are very good, the resistance to fatigue failure is very low. This is a characteristic common to most ceramics in general and bioactive ceramic materials in particular. Physiological tensile loading of sintered apatite implants will cause fatigue fracture within a few months (Seidelmann ea. 1982, de Groot 1981, Ritter 1974). This makes sintered hydroxyl-apatite implants unsuitable for load-bearing applications.

\section{Biocompatibility of calcium-phosphate ceramics}

There is well documented proof in the literature that sintered hydroxyl-apatite can form very tight bonds with living bone (Cook ea. 1986, Tracy ea. 1986, Klein 1984, Jarcho 1981 $\& 1977$, Denissen 1980). Only fixation, but no exact fit is required between implant and bone, because new bone grows into the spaces and fills them within a few weeks. The bond between implant and bone is usually so strong that the implant cannot be removed without fracturing the surrounding bone. This holds true for both unloaded and loaded implants, provided gross initial micromotion is prevented.

At the sub-microscopical level the bonding between hydroxyl-apatite and bone is that of direct apposition of bone on the implant surface (Tracy ea. 1986, Jarcho 1981, Denissen 1980). In the first few months there is an amorphous transition zone between hydroxylapatite implant and bone with a thickness between 100-300 nm (nanometer). This layer consists of amorphous vital bone without any fibrillar or cellular elements. With time this layer becomes thinner until after three months an ultimate thickness of 40 nanometer is reached. This is in the order of magnitude of the crystal size of hydroxyl-apatite (60 nanometer). The bone micro-crystals are arranged against the implant surface in a palisade like fashion, resembling the natural bonding between two bone fragments (Jarcho 1981). Bonding of hydroxyl-apatite does not only occur with bone, but also with epithelial and connective tissue. This makes them also suitable for percutaneous or permucosal implants (de Putter 1984 \& 1983). Biocompatibility characteristics of tricalcium-phosphate are similar to hydroxyl-apatite, although bone bonding seems to proceed somewhat more rapidly to hydroxyl-apatite than to tricalcium-phosphate (Klein 1983).

\section{Biostability of calcium-phosphate ceramics}

Since bone is continually subject to remodelling, which includes bone formation by osteoblasts and bone resorption by osteoclasts, one might expect the same phenomena to 
occur with calcium-phosphate ceramics. In general however, this is not the case. Biodegradation of calcium-phosphate ceramics occurs normally in two steps. At first, individual particles loosen from the implant surface by physico-chemical processes of dissolution. Only after this step can ingestion and intra-cellular dissolution of the ceramic particles occur. This second step is a cell-dependant metabolic process. Based on these kinetics one can make some general remarks:

The rate of chemical dissolution of ceramic particles is dependent upon the available surface. Increasing the porosity of the materials will increase resorption. Dense materials will have a very small available surface for physico-chemical resorption. For this reason they have low resorption rates.

The rate of dissolution is dependent upon the material of which the implant is made. Tricalcium-phosphate has a much higher resolution rate than hydroxyl-apatite.

The overall rate of degradation is dependant upon the first step of physico-chemical dissolution. When this step is inhibited cell-mediated degradation cannot occur.

When we compare the available implant surfaces for dissolution of a dense hydroxylapatite implant, which has a closed surface structure, with the multi-fragmented microcrystalline deposition of hydroxyl-apatite in natural bone with its crystal size of 5 by 20 by 60 nanometer, the differences in surface area are immense. This explains why dense hydroxyl-apatite of good chemical purity has a negligible degradation rate in vivo. Small impurities, crystallographic defects and vacancies or other materiological imperfections like micro-pores can however strongly affect degradation characteristics.

Experimental studies confirm that dense hydroxyl-apatite does not undergo any significant biodegradation once fixation to the bone is obtained (Holmes ea. 1986, Renooij ea. 1985, Hoogendoorn ea. 1984, Davidge 1984, Klein 1984 \& 1983, Jarcho 1981 \& 1977). In the first few months some 10-15 microns of an hydroxyl-apatite surface may dissolve in the process of acquiring bony union (van Blitterswijk ea. 1986). This is probably caused by the fact, that the surface layer is composed of less dense sintered particles and may contain some surface irregularities. This outer surface layer is therefore more susceptible to physico-chemical dissolution. Once fixation of dense implants to bone is obtained no further degradation can be observed. In the absence of bone bonding, hydroxyl-apatite shows very slow continued degradation at a rate of approximately 15 microns surfacethickness loss a year. Hydroxyl-apatite is chemically stable at pH-levels of higher than five. Also under clinical conditions hydroxyl-apatite seems to be stable (Patka 1985). Tricalcium-phosphate is resorbable at physiological pH-levels and therefore cannot be used for permanent implants.

\section{Clinical compatibility of calcium-phosphate ceramics}

In the clinical setting, hydroxyl-apatite has been in use since the late seventies as a bone grafting material in dental surgery (de Putter 1984 \& 1983, Denissen 1982, Tuinzing 1981), orthopaedic surgery (Patka 1985, Osborn 1985, Magerl ea. 1983, Ponssen 1983), maxillo-facial surgery (Roth ea. 1984, Kent ea: 1982), ear-nose-throat surgery (Grote ea. 1981), and plastic or other reconstructive surgery (Feenstra ea. 1983). Its excellent biological properties have been confirmed and no unfavorable biological reactions have been reported. 


\section{Conclusions}

The conclusion, drawn from the literature, is that sintered hydroxyl-apatite offers attractive biological properties of bone bonding with good longterm stability in vivo. The major drawback of hydroxyl-apatite is its poor fatigue strength. This precludes its use in tensile load-bearing applications like artificial hip or knee joints. Improvements in industrial technology can now overcome mechanical drawbacks of bio-active ceramics. Composites with a biocompatible surface of hydroxyl-apatite and an inner structure of metal for obtaining sufficient mechanical strength are required, if load-bearing parts of the skeleton are to be replaced. In dental surgery, prestressed artificial dental implants have become a clinical success (de Putter 1983). The implants are of simple cylindrical form, that can be prestressed by a central screw (van der Zel 1981). For more complex designs like artificial hip joints, this method is not suitable.

Implant coating (Bunshah 1981) seems to be an attractive alternative in obtaining a composite material that combines the inner strength of a metal with the biological surface characteristics of hydroxyl-apatite. The coating is manufactured using a plasma-spraying technique to be described in the next chapter. Due to refinements in technology, hydroxyl-apatite coatings on titanium metal substrates can be prepared of good chemical purity and mechanical strength. Hence, a completely new orthopaedic implant material is available.

For optimal performance in load-bearing applications, hydroxyl-apatite coatings should meet certain requirements:

- mechanical strength

- biocompatibility

- biostability

Metals in use for coating substrates, particularly titanium, have a long clinical history of implantation and need no special attention. The new development is the combination of the characteristics of hydroxyl-apatite and titanium. To be proved is, that this new composite material has better physical and biological characteristics than its individual components and that there are no significant adverse effects.

The following questions regarding hydroxyl-apatite coatings must therefore be discussed:

1 - Are the physical characteristics, including fatigue behavior, of hydroxyl-apatite coatings good enough for load-bearing orthopaedic applications?

2 - Is the biological behaviour of hydroxyl-apatite coated implants the same as that of sintered hydroxyl-apatite implants?

3 - Are there any adverse effects of the coating procedure on either hydroxyl-apatite, metal substrate or bone? 
Because there are no literature data available concerning the biological behaviour of hydroxyl-apatite coatings, several studies were performed to explore its usefulness in orthopaedic surgery.

The subject of this study is to evaluate the possible application of hydroxyl-apatite coatings in orthopaedic surgery, especially in artificial hip implants. This will be detailed in the following sections:

- preparation of hydroxyl-apatite coatings

- physico-chemical characteristics of hydroxyl-apatite coatings

- biological and mechanical interface characteristics with bone in a canine plug implant study

- evaluation of hydroxyl-apatite coatings under load-bearing in canine total hip replacement

- considerations for human total hip replacement using hydroxyl-apatite coatings

- early experience with human hydroxyl-apatite coated total hip replacement

- concluding remarks and summaries 



\section{Chapter three}

\section{Plasma-spraying and hydroxyl-apatite coatings}

Hydroxyl-apatite coatings must be strong and durable to be useful in hard tissue replacement under load-bearing conditions. Their static physical strength must be high enough to withstand the prevailing implant loading conditions and they must survive to longterm fatigue failure. The criteria is relevant for the hydroxyl-apatite coating and the bond strength of the coating to the substrate metal.

Because it is impossible to measure the actual implant-bone interface stress, we must either resort to indirect data relating to stresses in bone surrounding implants or calculate the actual implant-bone interface stress using finite-element techniques. Using strain gauge techniques at the outer surface of the proximal femoral bone, bone stresses up to 16.7 MPa have been reported with cemented types of hip prostheses (McBeath 1980). Because these stresses are measured at some distance from the implant, they have no direct correlation with the actual implant-bone interface stresses.

Calculations for the actual implant-bone interface stresses can be made with the use of finite-element-method (FEM) techniques. Results of these FEM calculations strongly depend on the assumptions for bone characteristics, choices for implant materials, implant geometry and method of implant fixation. Using general materials and geometrical characteristics for bone and implants, some general requirements can be set for hydroxyl-apatite coatings to be effective for implant fixation. Once more specific data are available regarding the mechanical and biological behaviour of hydroxyl-apatite coatings, the FEM technique can be more refined for specific applications.

As an initial guideline, finite-element data are available concerning interface stresses around specific types of non-cemented hip components (Huiskes 1987). Average stress peaks up to $9.6 \mathrm{MPa}$, with worst case peaks of up to $24 \mathrm{MPa}$, are calculated for the subchondral bone layer close to the first and last screw threads of acetabular screw rings (Huiskes 1987). On the femoral side, implant-bone interface stresses strongly depend on the rigidity of the femoral stem design. Using more flexible (elastic) stem designs, highest interface stresses occur around the proximal stem section with calculated values of up to 4 MPa under average loading conditions. In the case of more rigid femoral stem designs, the higher interface stresses, up to $3.2 \mathrm{MPa}$, occur at the distal stem interface section (Huiskes 1986). The interface stress values for non-cemented hip implants are generally higher as compared to those calculated for cemented implants under comparable loading conditions. Interface stresses of up to $1.4 \mathrm{MPa}$ are calculated for cemented implants, the localization of maximal stresses being in general the distal stem interface area, depending on geometrical design of the implant (Huiskes 1986). Because the values for interface stresses, calculated for current non-cemented implants tend to be lower in cases where there is rigid bonding between metal implant and bone (Huiskes 1987), these values could be used as preliminary assumptions for the maximum interface stresses, to be expected around hydroxyl-apatite coated implants.

Because there is thusfar no actual literature data on hydroxyl-apatite/titanium implantbone interface characteristics, the actual requirements for mechanical strength of 
hydroxyl-apatite coatings are still unknown. Once more specific data are available on actual interface bond strength values of hydroxyl-apatite coatings, finite-element calculations can be made for specific applications and choices of implant geometry, proving or disproving the concept of biological fixation by hydroxyl-apatite coatings. Requirements for fatigue characteristics are even more difficult to define. An individual, walking five kilometers a day experiences approximately one million cycles of hip joint loading a year. Ten million cycles, simulating ten years of hip joint loading, is an accepted standard for fatigue testing of implants (Ducheyne ea. 1986, Cook ea. 1984, Yue ea. 1984, Moore 1948).

The most important factors to determine the physical strength of hydroxyl-apatite coatings are:

\author{
thickness and density of coating \\ substrate metal \\ technique of plasma-spraying \\ chemical purity of hydroxyl-apatite powder
}

\title{
Thickness of hydroxyl-apatite coating
}

The initial thickness of a hydroxyl-apatite coating is a compromise between a number of limiting conditions. As mentioned earlier, in the first few months, some 10-15 microns of an apatite surface may dissolve in the process of acquiring bony union. This determines the absolute lower limit of coating thickness. Also, the thinner the coating the better its mechanical properties. When the coating becomes thicker, its mechanical characteristics gradually resemble more those of sintered apatite. Thick coatings are brittle and can easily chip off the substrate, as determined with some preliminary tests. This is comparable to the brittleness of thick glass, as compared to the flexibility of thin glass-fibers. A thick coating may reintroduce fatigue problems under tensile loading. From a practical point of view, a coating thickness between 30-80 microns appears a reasonable compromise. Further experiments in this study are carried out with hydroxyl-apatite coatings of $50 \pm$ 20 microns thickness. A coating thickness of 50 microns allows some degradation to occur during the initial phase of bony ingrowth, while probably still preserving a coating of sufficient thickness to be effective for implant fixation. The minimum required coating thickness for hydroxyl-apatite coatings under biological conditions is probably not too critical, as long as structural integrity of the coating is preserved at its outer surface. Bone bonding to hydroxyl-apatite coatings is a surface phenomenon, and therefore not very dependant on ingrowth to any required minimum depth. The ultimate optimum value for hydroxyl-apatite coating thickness still has to be settled after future experiments.

\section{Density of hydroxyl-apatite coating}

Completely dense coatings of hydroxyl-apatite have the highest mechanical strength.

Inducing micro- or macro-porosity drastically reduces both static and fatigue strength. A reduction in density of as small as $100 \%$ to $95 \%$ will reduce the mechanical strength of the coating to less than $50 \%$ of its original value. Also, porous calcium-phosphate ceramics show increased biodegradation rates. Both arguments are in favor of dense coatings. Moreover, porous structures are not really necessary for bone bonding to occur. The bone bonding to hydroxyl-apatite implants is a surface phenomenon and not dependent on a 
three-dimensional mechanical interlock with the implant, as is the case with porous-metal coatings.

\section{Metal substrates for coating}

Titanium and chrome-cobalt alloys are frequently used for orthopaedic implants.

Although both metals are theoretically suitable for apatite-coating, titanium is preferred for several reasons. At the elevated temperatures, used during plasma-spraying, titanium is chemically more reactive than chrome-cobalt. For this reason it is thought, the titaniumhydroxyl-apatite interface is stronger than the chrome-cobalt-hydroxyl-apatite interface. Another reason in preference for titanium is its more favorable modulus of elasticity. The modulus of elasticity for titanium (110.000 MPa) (Semlitsch 1983) is closer to that of bone (16-19.000 MPa) (Albright 1977) and less than half that of chrome-cobalt alloys (230.000 $\mathrm{MPa}$ ). Although the geometrical design of the implant is probably more important in the prevention of "stress-shielding", the modulus of elasticity of the implant material is still a significant factor. Both arguments provide enough justification to further investigate hydroxyl-apatite coatings on titanium substrates.

\section{Technique of plasma-spraying}

\section{Metal substrate}

The metal substrates investigated conformed to ASTM ${ }^{2}$ standards F-67 and F-136 titanium alloys. F-136 is a titanium alloy designated as Ti-6Al-4V ELI, indicating it contains 6 percent aluminum, 4 percent vanadium, balance titanium, for increased strength. In the presence of oxygen, titanium is immediately covered with a thin (10 nanometer) layer of titanium-oxide $\left(\mathrm{TiO}_{2}\right)$, giving titanium implants the surface characteristics of a bioinert metal-oxide ceramic. Both titanium alloys have widespread application in orthopaedic implants. Literature data (Lacout ea. 1984) show that titanium and its oxides may react with hydroxyl-apatite at elevated temperatures. This can occur in an oxidizing as well as in a non-oxidizing atmosphere. Table II shows the two possible chemical reactions that may lead to the desired chemical bonding between hydroxylapatite and titanium.

\section{Table II}

Reactions of titanium-oxide with hydroxyl-apatite

$$
\begin{aligned}
& 1 \quad(\mathrm{n}+1) \mathrm{Ca}_{10}\left(\mathrm{PO}_{4}\right)_{6}(\mathrm{OH})_{2}+\mathrm{n} \mathrm{TiO}_{2} \stackrel{\left(1000^{\circ} \mathrm{C}\right)}{\longrightarrow} \\
& \mathrm{n} \mathrm{CaTiO}_{3}+(\mathrm{n}-1) \mathrm{Ca}_{10}\left(\mathrm{PO}_{4}\right)_{6}(\mathrm{OH})_{2}+3 \mathrm{n} \mathrm{Ca}_{3}\left(\mathrm{PO}_{4}\right)_{2}+\mathrm{n} \mathrm{H}_{2} \mathrm{O}
\end{aligned}
$$
$2 \quad 2 \mathrm{TiO}_{2}$
$\left(850^{\circ} \mathrm{C}\right)$
$\left(\mathrm{Ti}^{4+}\right)\left(\mathrm{TiO}_{4}{ }^{4-}\right)$ incorporated in apatite lattice

\footnotetext{
${ }^{2}$ American Society for Testing and Matcrials
} 
To improve the bonding between titanium and hydroxyl-apatite, some kind of surface pretreatment is necessary. This consists of bead-blasting with alumina-oxide $\left(\mathrm{Al}_{2} \mathrm{O}_{3}\right)$ particles until a surface micro-roughness of $3-5$ microns is established. Subsequently, the metal surface is cleaned by both chemical and ultrasonic techniques. For improved coating bond it is necessary to preheat the implant surface with the plasma-flame in order to remove any unwanted organic deposits.

\section{Hydroxyl-apatite coating powder}

The powder particles used in the coating process are prepared in our laboratories. The starting powder is available commercially. Particle size of the raw material is too small for plasma-spraying. It has the correct chemical and crystallographic structure with a $\mathrm{Ca} / \mathrm{P}$ ratio of 1.67 (10/6), like hydroxyl-apatite of living bone. The $\mathrm{Ca} / \mathrm{P}$ ratio of the starting powder is an important criteria to determine its chemical purity and differentiates it from tricalcium-phosphate (TCP), which has a $\mathrm{Ca} / \mathrm{P}$ ratio of 1.5 . The use of starting powders with a $\mathrm{Ca} / \mathrm{P}$ ratio different from $10 / 6$ will result in ceramics of non hydroxyl-apatite structure. The crystal structure can be assessed by X-ray crystal-diffraction analysis and infrared spectrometry, both before and after plasma-spraying. To increase the particle size of the hydroxyl-apatite coating powder, it is first sintered to a dense hydroxyl-apatite ceramic using the technique described in the previous chapter. Its quality and chemical purity are then assessed by X-ray diffraction techniques. Subsequently, the dense solid ceramic is milled into fine particles until at least 90 percent of the particles is smaller than 50 microns. This is usually enough to prevent the blocking of the feeding pipes to the plasma-flame.

\section{Plasma-spraying technique}

The principle of plasma-spraying is known from industrial technology (Bunshah 1981). A DC-electric arc of high energy (400 Amperes at 100 Volts) is struck between two electrodes, while a stream of mixed gases passes through (Fig. 2). Both an argon-hydrogen mixture or nitrogen are suitable, depending on the required characteristics of the plasmaflame. Argon-hydrogen mixtures produce stronger coatings, but are more difficult to handle with large objects because of the small flame area. Additionally, argon-hydrogen mixtures are more expensive and dangerous because of the presence of the flammable

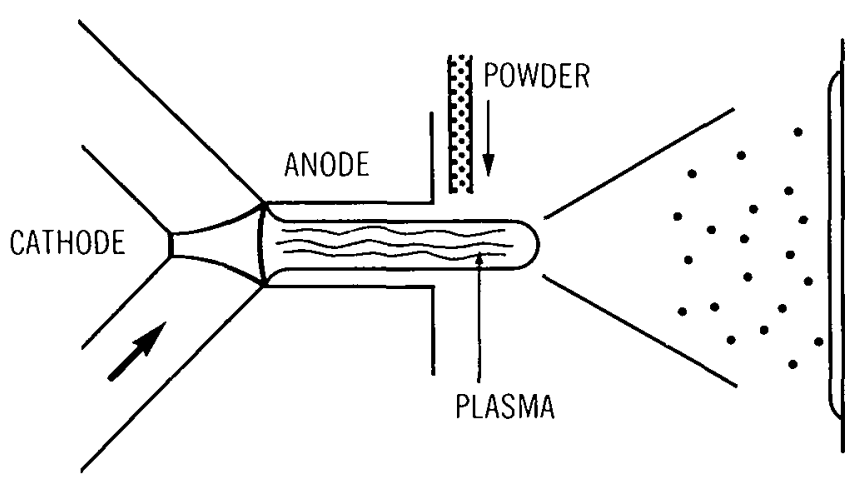

Fig. 2

Schematic representation of plasma-spraying procedure. 
hydrogen. The passage of the gas through the electric arc results in a ionized gas, called a plasma, of very high temperature, up to $30.000{ }^{\circ} \mathrm{C}$. Due to the high expansion following this temperature increase, the gas obtains a high speed, approaching the speed of sound. Hydroxyl-apatite powder particles of the appropriate size are then suspended in a separate carrier gas stream and fed into the plasma flame. A special powder port $\left(\right.$ Metco $\left.^{\circledR}\right)$ allows oblique injection of the hydroxyl-apatite particles at the far end of the plasma-flame, thereby lowering the risk of the particles being burned or evaporated. Due to the very high temperature the still relatively large hydroxyl-apatite particles explode into much smaller particles (1-5 microns) and start to melt at their surfaces. Because of the very high speed of the gas, the hydroxyl-apatite particles will stay in the plasma-flame for only a few milliseconds. If the individual hydroxyl-apatite particles remain too long in the very hot plasma-flame, they will evaporate or the crystal structure will undergo irreversible phase changes with loss of the specific hydroxyl-apatite characteristics. The critical temperature for this crystal phase transition is approximately $1300^{\circ} \mathrm{C}$. For optimal chemical bonding between hydroxyl-apatite and titanium the particles must arrive at the target surface just slightly molten at their surfaces. If on the other hand the temperature of the particles is too

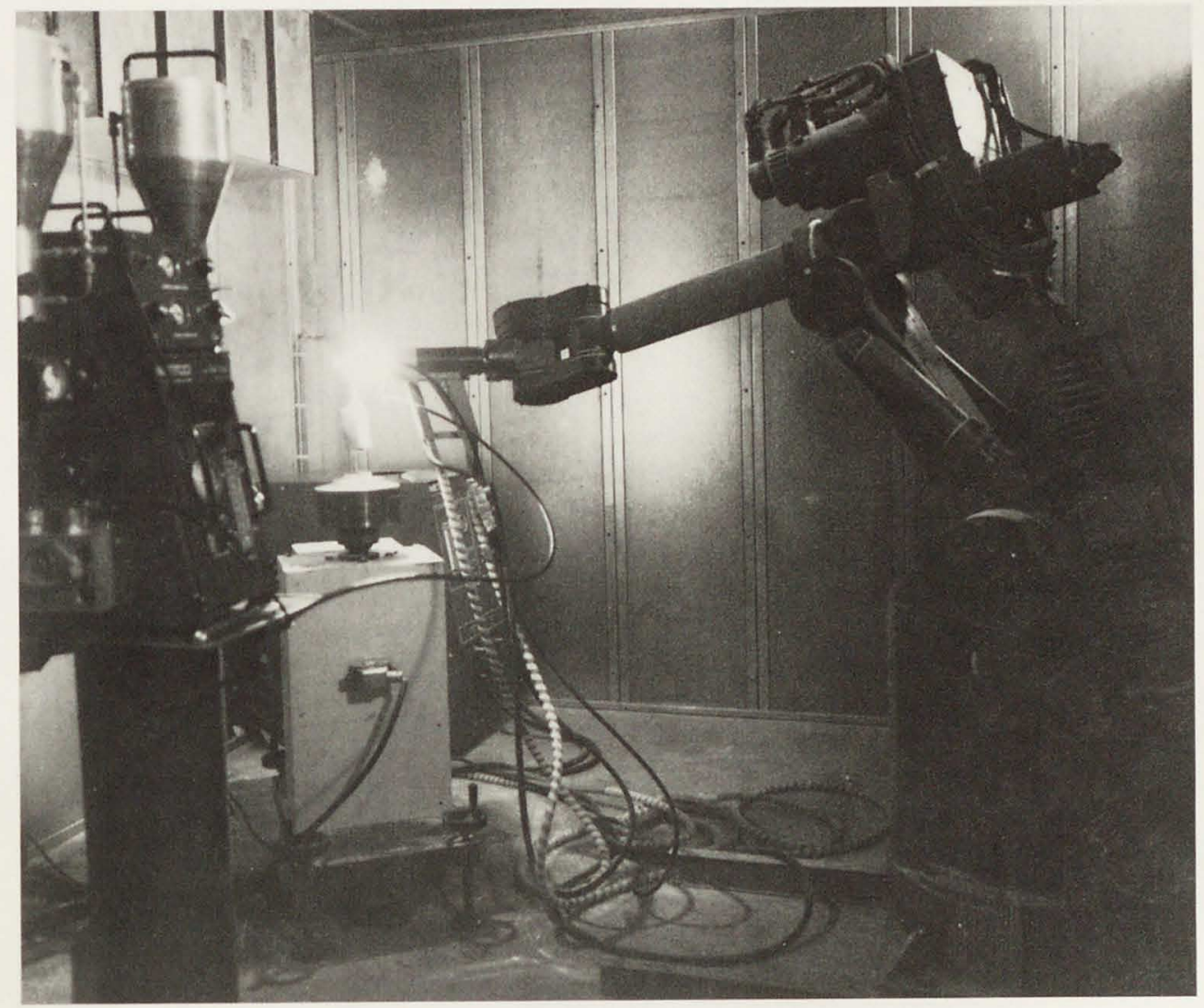

Fig. 3

Experimental set-up of equipment in use for plasma-spraying. 
low, while arriving at the target surface, sintering of the particles onto the metal surface will not occur. The resultant coating will then consist of only mechanically compacted particles without coherence and with excessive porosity. Although the visual appearance of such a coating, very white, may look attractive, the substrate bonding strength of such physical-deposition hydroxyl-apatite coatings is much lower as compared to hightemperature chemically sintered hydroxyl-apatite coatings. Dense coatings are gray and slightly transparent: dense apatite single crystals are translucent like glass.

Because of the very narrow temperature margins during the plasma-spraying procedure it is usually necessary to install a micro-computer with robot control of the plasma-flame nozzle to assure uniformity in quality and thickness of the resultant hydroxyl-apatite coating (Fig. 3). The coating is applied in several layers, each with a thickness of 5-10 microns. Surface irregularity is thereby in the order of magnitude of 5-10 microns. Each new layer will close the surface pores and irregularities of the lower layer. The resultant coating (Fig. 4) has a thickness of approximately 50 microns, of which the lower 30-35 microns are completely dense without micropores or other defects. The upper 10-15 microns surface layer may contain some microporosity (Fig. 5). This possibly plays a role in the slight decrease in coating thickness observed in the first months after implantation, as well as the differences in static strength of the hydroxyl-apatite coating under laboratory or biological conditions, as will be discussed later.

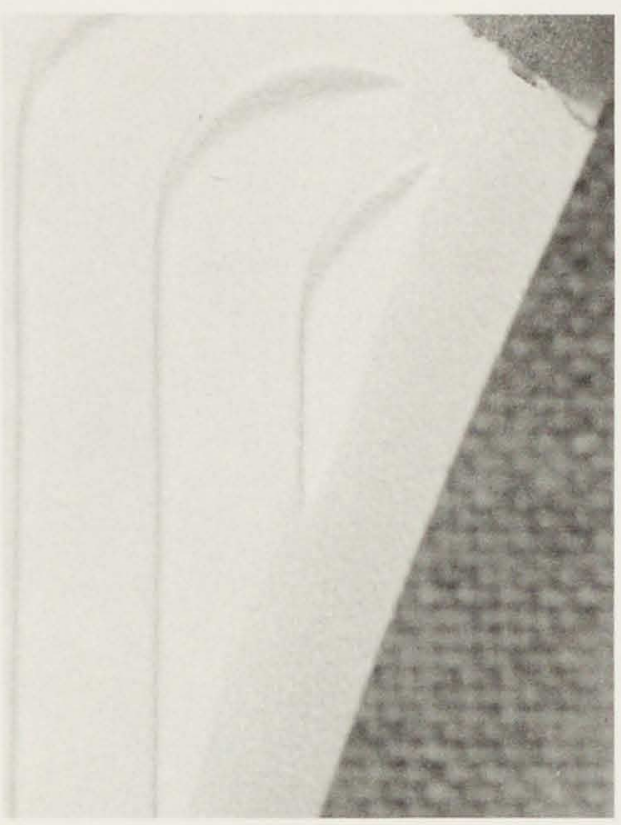

Fig. 4

Surface structure of hydroxyl-apatite coated implant.

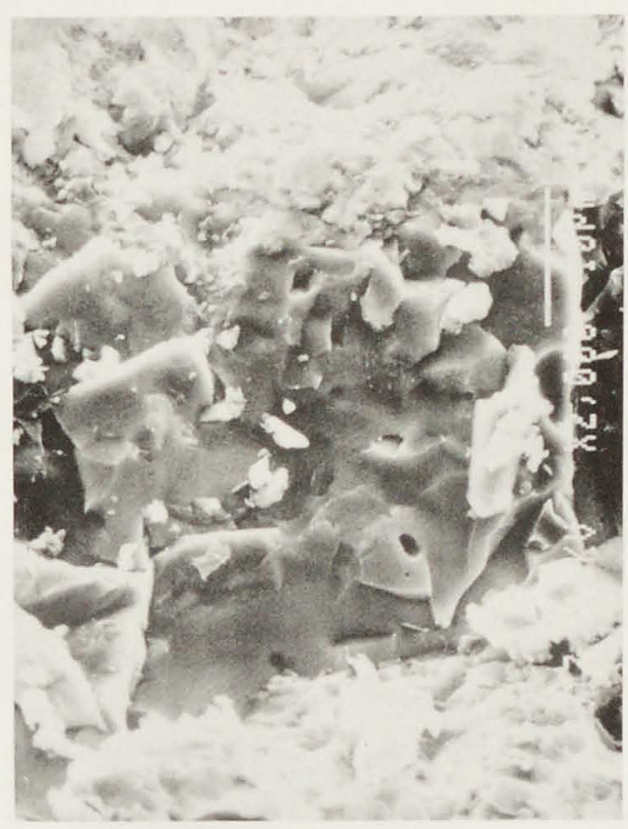

Fig. 5

Structure of surface and deeper layers of hydroxylapatite coating. (Scanning-electron photograph $\mathrm{x} 400)$. 


\section{Special handling problems of implants}

For implants to be coated partially, there is the additional problem of masking the areas to be protected from coating. Current femoral stems have a coating on the proximal half of the stem area, while the acetabular component has no coating on the central dome area. Consequently, the neck and distal stem area of the femoral component and the central dome area of the acetabular component have to be protected from both bead-blasting and plasma-spraying by adequate masks.

Masking during bead-blasting is accomplished with the aid of cold-molded resin masks with windows for the blasting areas. Masking during plasma-spraying is accomplished using windowed titanium masks of comparable design. Furthermore, the areas of the implant to be protected from coating have to be cooled to temperatures below $200^{\circ} \mathrm{C}$. Bare titanium surfaces exhibit an irregular blue discoloration if exposed to temperatures in excess of $200^{\circ} \mathrm{C}$. Although temperatures up to $980^{\circ} \mathrm{C}$ do not alter the metallurgical characteristics of titanium, irregular discolorations of implants, harmless as they are, renders them commercially unacceptable. 



\section{Chapter four}

\section{Physico-chemical analysis of hydroxyl-apatite coatings}

Several test samples including plates, plugs and hip implants were coated with hydroxylapatite using the plasma-spraying technique, described earlier. This chapter describes the procedures and results for testing of the hydroxyl-apatite coating. Both chemical and physical parameters of the hydroxyl-apatite coating and its titanium substrate are described. More specifically, the following parameters regarding hydroxyl-apatite coatings have been determined:

chemical characteristics of hydroxyl-apatite coatings

- chemical purity

physical characteristics of hydroxyl-apatite coatings

- thickness

- surface irregularity

- static bond strength in shear loading

- static bond strength in tensile loading

- fatigue strength

- effects on strength of sterilizing techniques

\section{Methods of testing}

\section{Chemical analysis}

Chemical purity of the hydroxyl-apatite coating material can be assessed by both X-ray diffraction technique and infrared light spectroscopy. $\mathrm{X}$-ray diffraction is the more accurate and therefore this technique was used for quality monitoring in this study. Some material was scratched off the surface of the coating with the aid of a diamond knife, crushed in an agate mortar and finely ground. The powder was put in a capillary and placed in the center of a Debye-Scherrer camera.

The diffraction spectrum was registered during a twenty hour exposition period to a cobalt lamp. The recorded lines were compared with the standards for tricalcium-phosphate and hydroxyl-apatite (X-ray ASTM standards 1973).

\section{Coating thickness and density}

The thickness of a hydroxyl-apatite coating can be assessed in a non-destructive way by an electronic measuring device, based on capacitive coupling between measuring electrode and target substrate. The maximum coating thickness is measured this way. The surface structure and density of the material can be inspected by means of light microscopy or scanning-electron microscopy.

\section{Static substrate bonding strength}

Static strength of the coating was determined in shear and tensile directions until failure. For uniformity of testing a standard test specimen geometry was designed (Fig. 6). For shear testing, each specimen consisted of a $12-\mathrm{mm}$. diameter test mandrel and was fabricated from the same titanium alloy as the coating test sample substrate. Each mandrel was centered in an annular machined steel housing with a slip-fit guide peg and hole. 

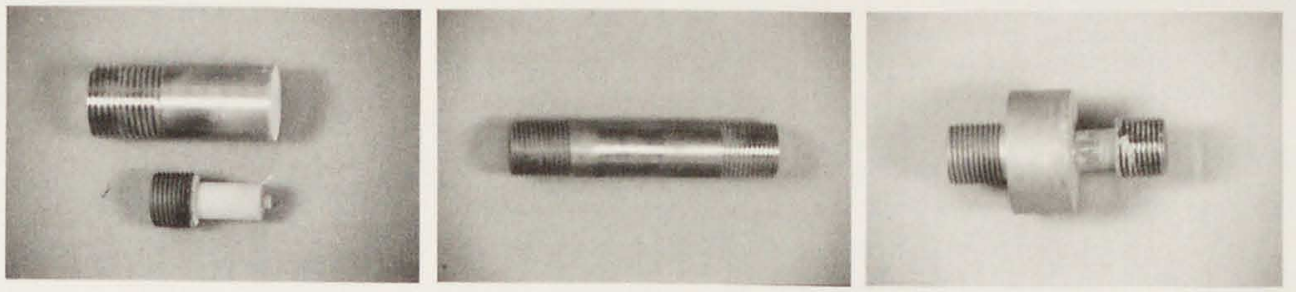

Fig. 6

Test samples for determination of tensile and shear strength of hydroxyl-apatite coating.

Fig. 7

Assembled test specimen for determination of tensile strength of hydroxyl-apatite coating.

Fig. 8

Assembled test specimen for determination of shear strength of hydroxyl-apatite coating.

Fig. 9

Installation of assembled test specimen for shear testing in test machine.

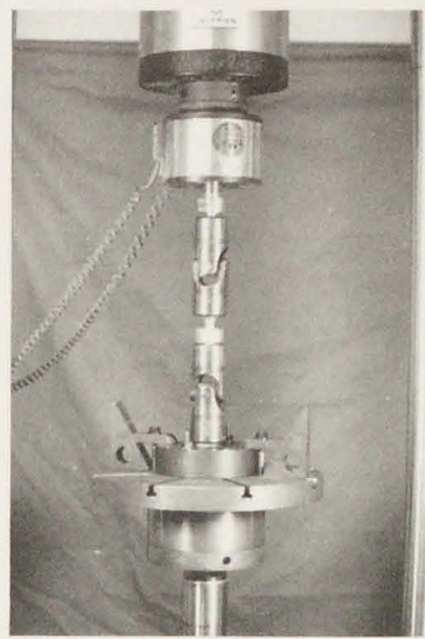

Around the circumference of the distal $1 \mathrm{~cm}$. of the test samples a hydroxyl-apatite coating was applied, using the techniques described earlier. For tensile testing a mandrel of comparable design was used, the difference being, that the coating was applied at its distal surface, instead of around its periphery. Thirty specimen were prepared. During assembly of the mandrel/housing pairs they were fixed together with the aid of an epoxyresin and mounted in an Instron ${ }^{\circledR}$ testing machine (Fig. 7, 8 and 9). The specimen were distracted with a crosshead speed of $1 \mathrm{~mm} . / \mathrm{min}$, recording the load displacement curves.

\section{Fatigue testing}

Fatigue tests were performed according to H.F. Moore (Moore 1948). The test samples consisted of circumferentially coated tubular structures, the ends of which were mounted under a variable angle of up to three degrees in the testing machine. By rotating the specimen, cyclic loading was obtained under fully reversed loading conditions. Fatigue testing of the coating was then performed according to Moore with tensile/tensile loading of $100-6000$ Newton in the fully reversed bending mode with cyclic loading during ten million cycles or until failure (Moore 1948). Additionally, functional fatigue studies were performed on fully hydroxyl-apatite coated femoral stems and non-coated controls. The purpose was to measure any deterioration in fatigue strength of the substrate metal during the course of the plasma-spraying procedure. Fully hydroxyl-apatite coated femoral stems, with non-coated control stems, were mounted in a three-point bending set-up (Fig. 10). The loading angle towards the implant neck was $62^{\circ}$ and the specimen were subjected 


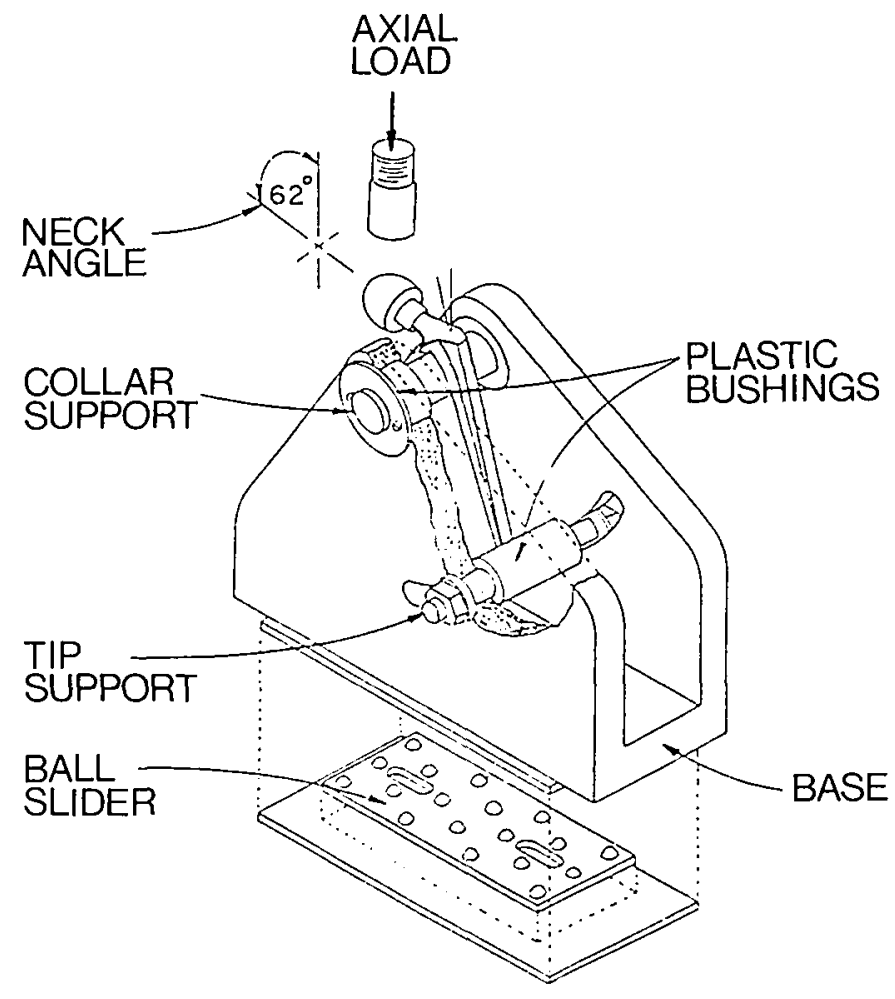

Fig. 10

Configuration for fatigue testing of hydroxyl-apatite coated femoral prostheses.

to cyclic loading up to 6000 Newton during ten million cycles according to Moore. Their mechanical strength was subsequently determined, providing data on mechanical strenght of femoral stems both with and without hydroxyl-apatite coating (Moore 1948).

\section{Sterilizing techniques}

Tests were repeated after sterilization of hydroxyl-apatite coated samples by:

- ethylene-oxide during 8 hours at $60^{\circ} \mathrm{C}$

- 2.5 megarad minimum gamma-irradiation

\section{Results}

\section{Chemical purity}

As determined by X-ray diffraction techniques, the hydroxyl-apatite coating consists for approximately 90 percent of pure hydroxyl-apatite material. The remainder are noncrystalline calcium-phosphate ceramics of non hydroxyl-apatite nature. 


\section{Physical testing results}

\section{Coating thickness and density}

The thickness of the coating can be controlled with an accuracy of \pm 20 microns. Implants with a coating thickness outside the $50 \pm 20$ microns range are discarded. Surface irregularity is in the order of magnitude of 5-10 microns. The upper 10-15 microns of a hydroxyl-apatite coating may contain some surface porosity, but the lower 30-40 microns are of a denser structure (compare fig. 5).

\section{Static bond strength}

Testing of the coating was done by shear and tensile loading. The results of the static mechanical testing are summarized in table III. Tensile loading resulted in (a) failure at approximately 60-70 MPa and (b) failure usually occurred at the interface epoxy-apatite or within the epoxy layer. Tensile shear strength measurements led to the following results: (a) shear failure at about 20-25 MPa and (b) failure occurrence mostly (more than 50\%) at the interface epoxy-apatite. Because failure generally occurred at the epoxy-apatite interface, the reported values represent minimum values.

\section{Fatigue characteristics}

Using the Moore specimens and the rotating beam fatigue tests with tensile-tensile loading of 100-6000 Newtons, there was no fatigue failure of the hydroxyl-apatite coating after ten million cycles of implant loading.

Table IV displays the results of the fatigue tests according to Moore on hydroxyl-apatite coated and control stems.

Table III

Minimum static mechanical strength of hydroxyl-apatite coatings.

Static tensile strength $\quad>60-70 \mathrm{MPa}$

Static shear strength $\quad>20-25 \mathrm{MPa}$

\section{Table IV}

Mechanical strength of hydroxyl-apatite coated and non-coated femoral stems.

\begin{tabular}{lll}
\hline $\begin{array}{l}\text { Alloy } \\
\text { (ASTM standard) }\end{array}$ & No of samples & $\begin{array}{l}\text { Mechanical strength } \\
\text { MPa } \pm \text { SD at } 10^{7} \text { cycles }\end{array}$ \\
\hline Titanium F-67 & 5 coated & $243 \pm 29.2$ \\
Titanium F-67 & 5 uncoated & $250 \pm 32.0$ \\
Titanium F-136 & 5 coated & $521 \pm 57.6$ \\
Titanium F-136 & 5 uncoated & $556 \pm 53.5$ \\
\hline
\end{tabular}

\section{Methods of sterilization}

With the investigated methods of sterilization, i.e. ethylene-oxide and gamma irradiation, there is no detectable difference in physical strength of the hydroxyl-apatite coating. 


\section{Discussion}

With mechanical testing a tensile bond strength of $65 \mathrm{MPa}$ and a shear strength of $25 \mathrm{MPa}$ was obtained for the hydroxyl-apatite coating. Although there is no precise information regarding the actually required bond strength of hydroxyl-apatite coatings, the recorded values appear good enough in comparison to bone strength to justify further experiments. Because failure during mechanical testing predominantly occurred at the epoxy-apatite interface, the recorded rates for mechanical strength represent minimum values.

Determination of coating strength should therefore be repeated under conditions resembling more those of clinical implantation of hydroxyl-apatite coatings. Nevertheless, the obtained rates for static bond strength and fatigue behaviour during mechanical testing appear sufficient to preclude the risk of loosening of the coating from the substrate metal. The major disadvantage of bulk hydroxyl-apatite ceramics, namely their susceptibility to fatigue fracture, is not applicable to 50 micron thickness coatings of hydroxyl-apatite on titanium substrates.

There is no measurable difference in mechanical strength values of coated and non-coated titanium stems. The results confirm that the coating process does not alter the metallurgical characteristics of the substrate metal with respect to fatigue behaviour. This is also theoretically conceivable. The critical temperature for beginning phase transitions and thereby loss of metallurgical characteristics for titanium alloys $\left(980^{\circ} \mathrm{C}\right)$ is higher than the actual temperatures reached at the implant surface during plasma-spraying.

Furthermore, there is no difference in mechanical data between specimens sterilized using either ethylene-oxide or gamma-irradiation. The investigated methods of sterilizing do not alter the properties of the hydroxyl-apatite coating. There is however some precaution with steam-autoclaving. Hydroxyl-apatite coatings are prepared at temperatures in excess of $1000^{\circ} \mathrm{C}$. This means that the autoclaving temperature of $144^{\circ} \mathrm{C}$ represents no problem in itself. The rate of temperature increase and decrease before and after sterilization can theoretically have some influence on coating bond strength, because of the differences in thermal expansion rates of titanium and hydroxyl-apatite. With rapid temperature changes there is theoretically some risk of the coating chipping off the metal, although this has not yet been observed in practice. In clinical practice, hydroxyl-apatite coated implants are sterilized using gamma-irradiation.

The conclusion of the physico-chemical testing is that hydroxyl-apatite coatings on titanium substrates have good chemical purity, strength and substrate bonding. Also the fatigue problem with sintered apatite implants is eliminated.

Results of our tests conform to other recent literature reports, although there are differences in observed coating bond strength values (Ducheyne ea. 1986, Kay ea. 1986, Lacefield 1986). This is explainable by the differences in plasma-spraying technique and its fine-tuning for optimum results. Some authors report the use of low temperature spraying techniques (Osborn 1987). This does not cause enough coherence between the individual particles of the coating. This again necessitates the use of much thicker coatings (200-500 microns) that have lower mechanical strength and inferior fatigue characteristics. 
From a materials point of view, the concept of hydroxyl-apatite coating using high temperature plasma-spraying techniques provides good justification for application with orthopaedic implants. 


\section{Chapter five}

\section{Cadaver implantation and retrieval of hydroxyl-apatite coated implants}

The previous chapter detailed the physico-chemical characteristics of hydroxyl-apatite coatings on titanium substrates from a laboratory point of view. This in itself provides enough justification for use of hydroxyl-apatite coatings in orthopaedic implants. In order to obtain clinical feedback from these laboratory data, two cadaver implantations were done. The object was to implant hydroxyl-apatite coated total hip prostheses and after subsequent retrieval inspect the implants for any damage to the coating.

\section{Methods}

Total hip implants of the Omnifit ${ }^{\circledR}$ system (Osteonics ${ }^{\circledR}$ ) were coated with hydroxylapatite. A surgical procedure, similar to a total hip replacement operation was performed on a fresh cadaver. Instrumentation for the acetabular screw-cup consisted of spherical reamers with $2 \mathrm{~mm}$. increments in size, while after final sizing the area for the screw thread was prepared with the aid of the appropriate conical reamer. A self-tapping hydroxylapatite coated screw ring was inserted to its full extent, while maximal torque was applied to the inserter.

Subsequently the screw-ring was screwed out and retrieved to the laboratory for analysis (Fig. 11). A corresponding procedure was performed on the femoral side. With the aid of conical intra-medullary reamers and corresponding calcar rasps, the proximal femur was prepared. After final sizing a slightly oversized Omnifit ${ }^{\circledR} \quad \# 10$ stem was inserted in the femoral shaft until flush with the resection level and subsequently extracted for inspection (Fig. 12). After retrieval the implants were ultra-sonically cleaned in alcohol and photographed.

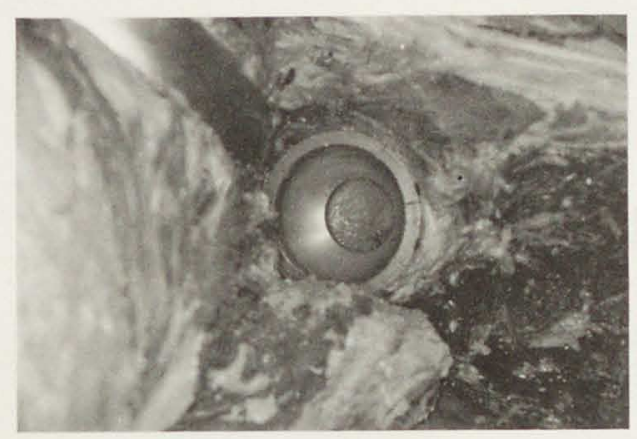

Fig. 11

Cadaver implantation of hydroxyl-apatite coated acetabular screw ring.

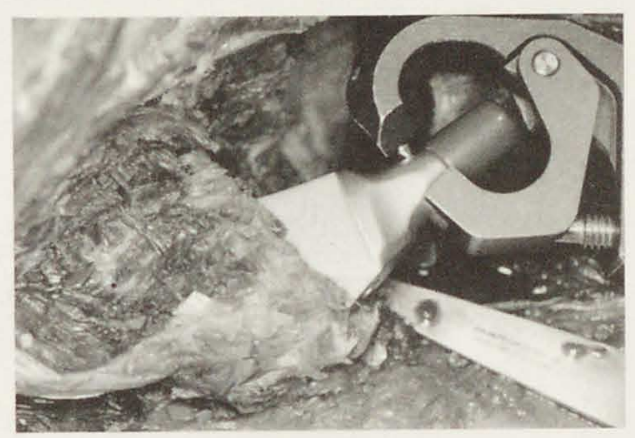

Fig. 12

Cadaver implantation of hydroxyl-apatite coated femoral stem, prior to full seating of prosthesis. 


\section{Results}

Gross inspection of the retrieved specimen did not show any significant damage to the coating on the femoral stem (Fig. 13).

There were only some superficial scratches on the medial proximal surface of the stem, but nowhere any visible more extensive loss of coating material. Also more refined microscopic examination of the coating surface did not provide additional information. Visual inspection of the acetabular screw-ring showed some full-thickness loss of coating at the starting points of the self-tapping screw-thread and at some places on the top of the screw-thread itself (Fig. 14). The areas outside the screw-threads and the inner aspects of the screw-thread itself were completely intact. Microscopical inspection confirmed these findings. The total area with loss of coating on the acetabular component was calculated as less than one percent.
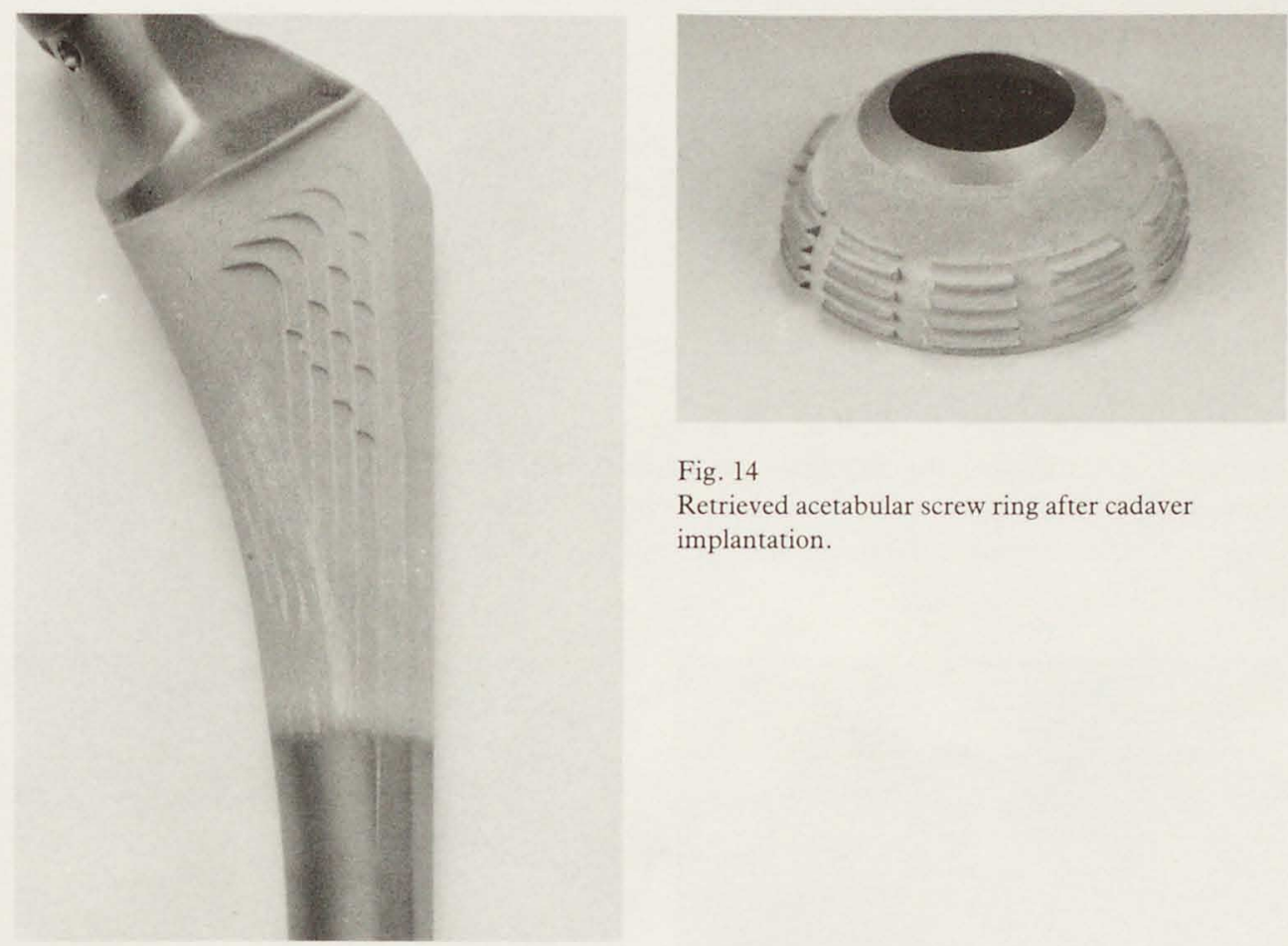

Fig. 14

Retrieved acetabular screw ring after cadaver implantation.

Fig. 13

Retrieved femoral stem after cadaver implantation. 


\section{Discussion}

In spite of the high forces that are required to insert a slightly oversized femoral stem into the medullary canal of the femur, no damage to the coating than some superficial scratches was observed. On the acetabular side there was some full-thickness loss of coating observed near the starting points of the screw-thread. Because the screw-thread is selftapping, very high friction forces can be expected in these areas during insertion, as well as on the top side of the screw-thread. The total area with damage to the coating was still less than one percent on the acetabular side and nil on the femoral side.

In conclusion, the overall rate of damage to the coating during insertion is very low and within acceptable limits. Moreover, there need be no fear of unfavorable biological effects of any loosened hydroxyl-apatite particles from the coating, should this occur to any significant extent.

The loose particles will be incorporated into the bone, just as has been described for sintered hydroxyl-apatite material.

The overall results of this cadaver retrieval study support our laboratory data regarding the physical strength of the hydroxyl-apatite coating, as prepared in our laboratories. The results of these implantations have been used to refine the configuration and tuning of the plasma-spray equipment. 



\section{Chapter six}

\section{Plug implant study}

The application of hydroxyl-apatite coatings for orthopaedic implants is quite new.

Therefore, little knowledge exists concerning its behaviour with bone. Although there are many analogies with sintered hydroxyl-apatite, some aspects might be new. The mechanical characteristics of hydroxyl-apatite coatings deserve special attention, since they differ markedly from sintered hydroxyl-apatite ceramics. The biological characteristics should, at least theoretically, not differ too much from sintered hydroxylapatite. Only the surface characteristics are relevant in this aspect because the hydroxylapatite coating has a dense structure. Nevertheless, it appears necessary to repeat some of the experiments that have been carried out with solid sintered hydroxyl-apatite implants with hydroxyl-apatite coated implants. Additionally, in vivo testing of hydroxyl-apatite coated implants appears more suitable for the determination of the ultimate mechanical strength of the coating, as discussed in chapter four.

This chapter describes the determination of elementary biological and mechanical interface characteristics of hydroxyl-apatite coatings to bone. A simple plug design serves this purpose. With implants of more complex design, like hip prostheses, it is much more difficult to quantify exactly the mechanical interface characteristics. Although the reliability of push-out tests for establishing interface-strength values is in itself questionable, its application can still provide evidence for the principles involved. The absolute values to be obtained are very dependant on experimental set-up, and should therefore not be taken too literally. The plugs in our study were implanted into dog femora. After follow-up, a mechanical, radiological and histological evaluation was performed.

\section{Materials and methods}

Cylindrical rods of standard Ti-6Al-4V titanium alloy were prepared, measuring 4.5 by 6 $\mathrm{mm}$. A hydroxyl-apatite coating of 50 micron thickness was applied using the plasmaspray technique, described earlier (Fig. 15). Results with non-coated titanium plug implants of same dimensions from a similar study were used as controls (Ronningen ea. 1984).

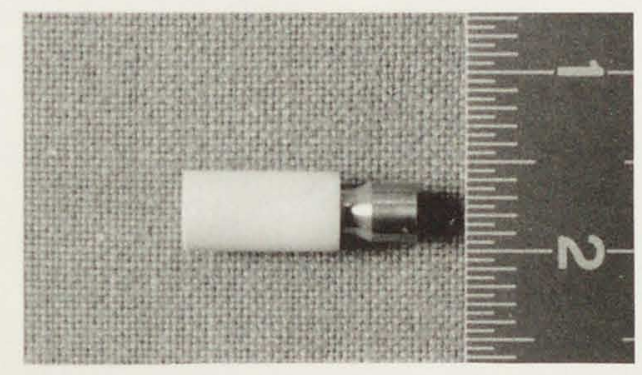

Fig. 15

Hydroxyl-apatite coated transcortical plug implant. 
Using sterile surgical techniques, the plugs were inserted into predrilled holes in the lateral cortex of adult canine (labrador) femora. To study exclusively the bone bonding properties of the material, the holes were slightly oversized $(4,7 \mathrm{~mm})$. This allowed the plugs to move in and out, but without undue laxity. The initial plug-bone interface strength therefore is zero. A total of 48 hydroxyl-apatite coated plugs were inserted into the femora of 8 dogs ( 3 plugs per femur). There were no surgical complications. After follow-up periods of 6 weeks, $3,6,12$ and 24 months the dogs were sacrificed using a lethal dose of thiopental. After explant radiography, the femora were used for histological examination and mechanical testing.

Each femur was sectioned in 3 pieces, each one with one plug. After removal of excess soft tissue the specimens were placed in formaldehyde to prevent biological degradation. Two of these plugs were used for mechanical testing. These were sectioned longitudinally, to gain access to the intramedullary part of the plug. The bone pieces were positioned in a testing jig to allow accurate lining of the loading axis of the test machine with the long axis of the plug (Fig. 16 and 17). The plugs were pushed out from the surrounding bone using an Instron ${ }^{\circledR}$ test machine with a crosshead speed of $1 \mathrm{~mm} / \mathrm{min}$. The maximum force to loosen the implant was calculated from the load-displacement curves.

Calculations for interface stress are very questionable and very dependant on experimental setup and assumptions for bony contact area. To allow some comparisons with literature data to be made, both the absolute values for push-out force as well as the relative interface shear strength values are provided in the tables. To calculate the shear strength of the

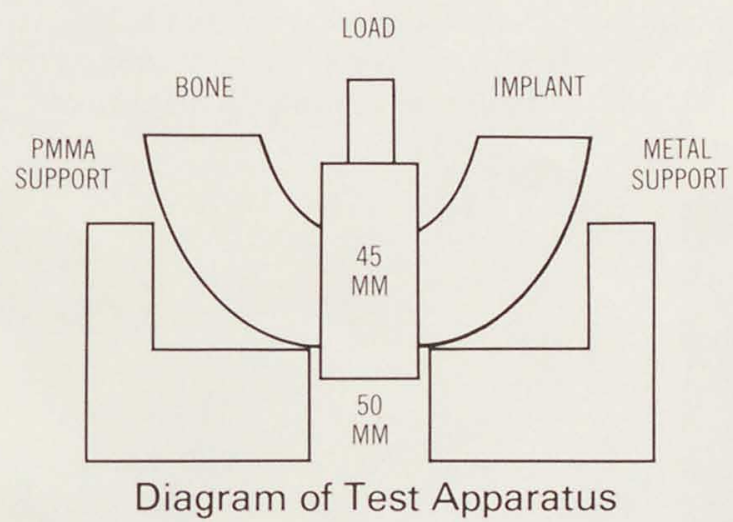

Fig. 16

Schematic configuration for push-out testing of transcortical plug implants.

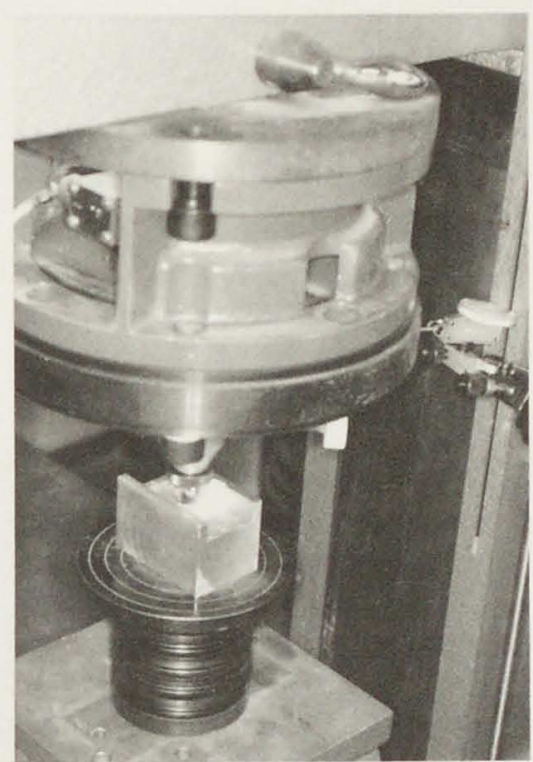

Fig. 17

Set-up for push-out testing of transcortical plug implants. 
interface, the push-out force was divided by the total plug/bone contact area, using the formula:

$$
\text { strength }=\frac{\text { push-out force }}{\text { contact area }}=\frac{\text { force (Newton) }}{\pi^{\star} 4.6^{\star} 1.75}
$$

The bone contact area was calculated using the formula Area $=\pi \star 4.6 \star 1.75$, where $4.6(\mathrm{~mm}$.) is the diameter of the plug implant with the coating and $1.75(\mathrm{~mm}$.) is the thickness of the femoral cortex around the plug implant. Because of the bone overgrowth on each plug and the questionable load-carrying capability of the overgrowth, a standardized cortical thickness of $1.75 \mathrm{~mm}$. was used in each shear strength calculation. At the two year follow-up interval four additional plugs were pushed-out of the bone immediately after sacrifice of the dogs, because of some concern about time delay or preparation artifacts (formalin) on mechanical strength of coating and bone.

One implant from each femur was selected at random and left intact for histological examination. After additional formalin fixation and alcohol dehydration the implants were embedded in polymethyl-methacrylate. Next, 50 micron thickness sections were prepared using a lowspeed diamond cutting wheel with cooling. From a few slices the metal was removed by manipulation under the microscope. Usually, this leaves the coating along the bone. From these slices 5 micron thickness sections were prepared that allow better cellular resolution than the 50 micron metal containing sections. Tissue staining was performed with basic fuchsine, alcian-blue, Masson-trichrome or haematoxyline-eosine. Transmission light and polarization microscopy were applied together with microradiography for analysis of the sections. One plug of a dog at the one year interval was reserved for scanning-electron microscopy. The bone was fixed with glutar-aldehyde and embedded in polymethyl-methacrylate, necessary for proper sectioning. In order to process these sections further, the polymethyl-methacrylate has again to be removed. This can be accomplished by alternating ultrasonic cleaning in 2-methoxy-aethylacetat and $90 \%$-alcohol (Brill ea. 1987). After applying a thin gold vapor-layer, analysis was carried out on a Philips ${ }^{\circledR}$ scanning unit.

Histology was reviewed with the following objectives:

character of coating-bone interface

response with cortical and trabecular bone

quality and quantity of new bone formation

response of bone-marrow (biocompatibility)

condition of hydroxyl-apatite coating

\section{Results}

\section{Visual inspection}

All coated plug implants appeared well incorporated into the bone (Fig. 18). There was invariably upgrowth of bone visible along the protruding part of the plug up to the external ending of the hydroxyl-apatite coating. The trabecular bone around the intramedullary part of a hydroxyl-apatite coated plug often displayed densification to bone of cortical nature (Fig. 19). There were no macroscopically loose implants at any follow-up period. 


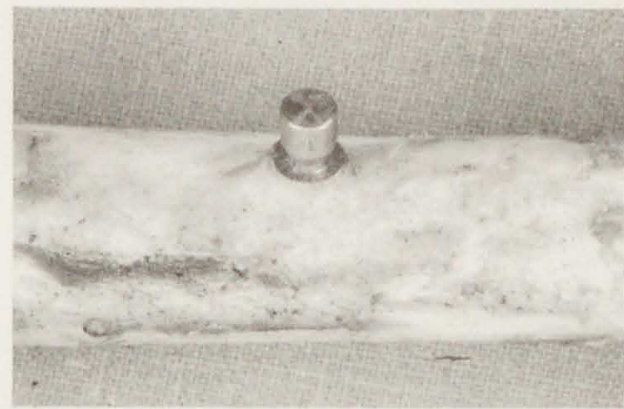

Fig. 18

Bony encapsulation of transcortical plug implant at three months, periosteal side.

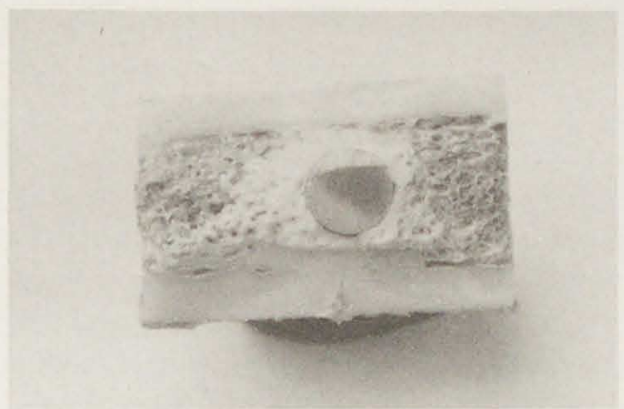

Fig. 19

Proliferation and densification of trabecular bone around hydroxyl-apatite coated transcortical plug implant in femur at three months, endosteal side.

\section{Explant radiography}

All coated implants displayed proliferation of bone along the protruding periosteal and endosteal part of the implant (Fig. 20). This was already visible at six weeks and became more prominent at longer follow-up periods (Fig. 21 and 22). Without protrusion of part of the hydroxyl-apatite coating from the bone there was no additional bone proliferation. The average increase in thickness of the bone at the periosteal as well as endosteal side of the plug was approximately $1.5-2 \mathrm{~mm}$. After three months follow-up, the whole endosteal as well as periosteal part of the plug was invariably completely covered with bone.

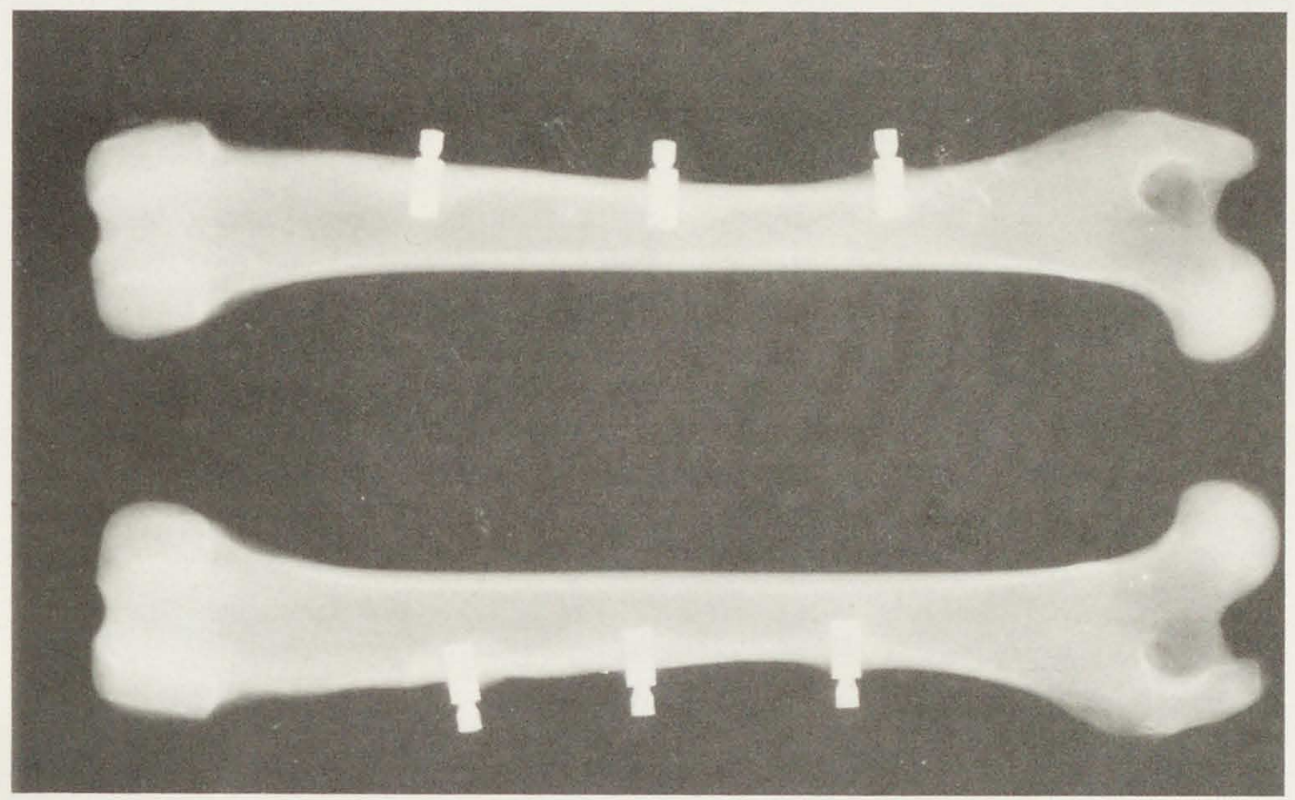

Fig. 20

Explant radiograph of femora with transcortical plug implants at six weeks. 


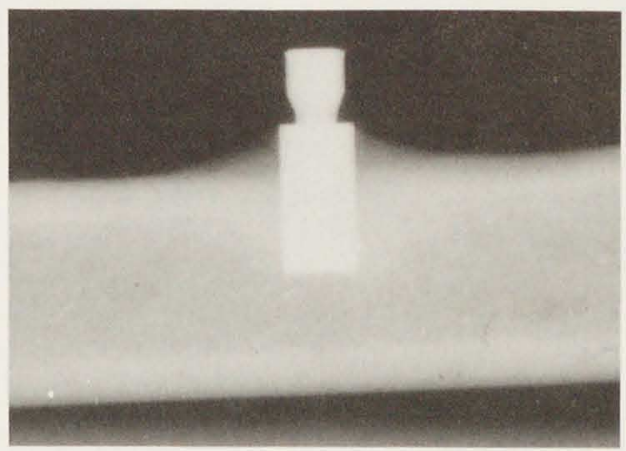

Fig. 21

Detail of explant radiograph of transcortical plug implant at six weeks follow-up.

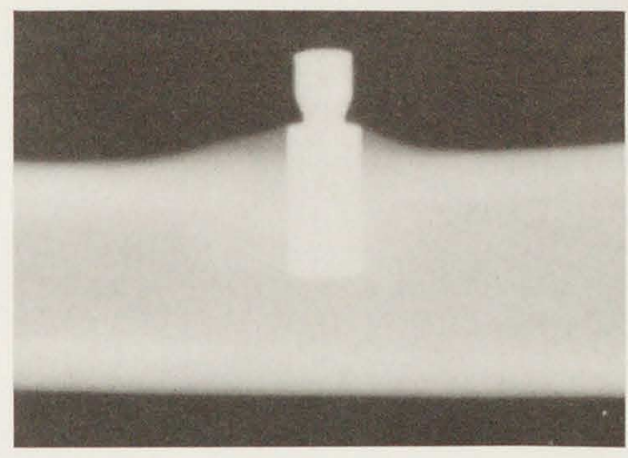

Fig. 22

Detail of explant radiograph of transcortical plug implant at six months follow-up.

\section{Mechanical testing}

The push-out data are summarized in table V. After 6 weeks there was already a mean interface shear strength of $48.5 \mathrm{MPa}$. A mean implant bone interface shear strength of 54 $\mathrm{MPa}$ was acquired after 3 months. Up to two years follow-up, the implant-bone interface shear strength remained at this level with plugs in conventionally prepared bone. Based on paired $\mathrm{T}$-test calculations, the differences in values obtained after three months and two years are not statistically significant at the $\mathrm{p}<0.05$ significance level, although the numbers are probably too small to provide hard proof of any differences.

The average of values obtained after the third month, was therefore $58 \mathrm{MPa}$. With fresh bone an average plug/bone shear strength value of $29.7 \mathrm{MPa}$ was obtained after two years follow-up. Values for plug-bone interface shear-strength, measured directly after sacrifice, proved thus to be approximately one half as compared to those obtained somewhat longer after sacrifice, where time delay and/or preparation artifacts can give distortion of results.

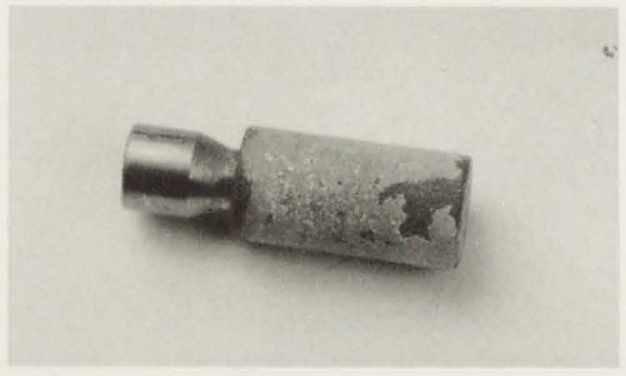

Fig. 23

Hydroxyl-apatite coated transcortical plug implant after push-out testing at three months. Failure predominantly within hydroxyl-apatite layer and to a lesser degree at coating-substrate interface.

\section{Failure mode}

The failure occurrence at the implant bone interface was predominantly within the hydroxyl-apatite coating itself (about $70 \%$ of surface area) and over the remainder of the surface area at the metal-coating interface (Fig. 23). After dislodging the implant, there was still some $60-70 \%$ of the maximum force necessary to further move the implants out of their holes. 
Table V

Average plug-bone interface strength with time $(\mathrm{MPa})$

\begin{tabular}{llll}
\hline $\begin{array}{l}\text { follow-up } \\
\text { weeks }\end{array}$ & $\begin{array}{l}\text { number of plugs } \\
\mathrm{nr}\end{array}$ & $\begin{array}{l}\text { force } \\
\text { Newton } \pm \mathrm{SD}\end{array}$ & $\begin{array}{l}\text { shear strength } \\
\text { MPa(megapascal) }\end{array}$ \\
\hline 6 & 8 & $1270 \pm 60$ & 49.5 \\
12 & 8 & $1360 \pm 70$ & 55.1 \\
25 & 8 & $1540 \pm 50$ & 62.1 \\
52 & 3 & $1440 \pm 40$ & 58.6 \\
104 & 4 & $1380 \pm 60$ & 56.3 \\
104 & 4 fresh & $720 \pm 70$ & 29.7 \\
\hline
\end{tabular}

\section{Histology}

Histological sections make clear that defects in the periosteum and bone around the implant fill with bone within 6 weeks (Fig. 24). Also, the periosteal tissue shows excellent adaptation to the hydroxyl-apatite coating (Fig. 25). Periosteal and endosteal bone proliferate along the protruding ends of the plugs in and out of the femur (Fig. 26 and 27). In the cortical area, bone is in very close contact with the implant, without any interposition of fibrous tissue. It shows remodelling with many active osteoblasts and osteoclasts along the lacunae. Not only mature osteocytes, but also numerous young osteoblasts and complete Haversian systems are seen in direct contact with the hydroxylapatite coating. Their osteoid is directly apposited on the coating (Fig. 28 and 29).

The transition area of hydroxyl-apatite coating to bone is of special interest. High magnification views $(1000 \mathrm{x})$ show some darkening of bone in this area with minute speckled particles in some places and smooth transitions under most circumstances (Fig. 30). In polarized light, the structure and orientation of the bone trabeculae is readily visible. Their endings on the hydroxyl-apatite coating have various angles to the implant surface, varying from perpendicular to almost parallel.

The thin sections, with the metal of the implant removed, show a similar picture. There are clusters of osteoblasts in close proximity to the coating, their osteoid spreading out towards the hydroxyl-apatite coating. The aspect of both nuclei and cytoplasma is completely normal. Also, the multinuclear osteoclasts exhibit no specific alterations from their normal appearance.

Initially, in the first six weeks, the architecture of the bone is lamellar and woven. With time the bone matures and becomes denser. Ultimately, after three to six months, the bone in contact with the hydroxyl-apatite coating is of dense cortical nature around the major part of its bony envelope.

In the intramedullary cavity, endosteal bone proliferates around the implant and manifests a normal trabecular structure. In this intra-medullary part of the femur boneresponse appears less prominent. 


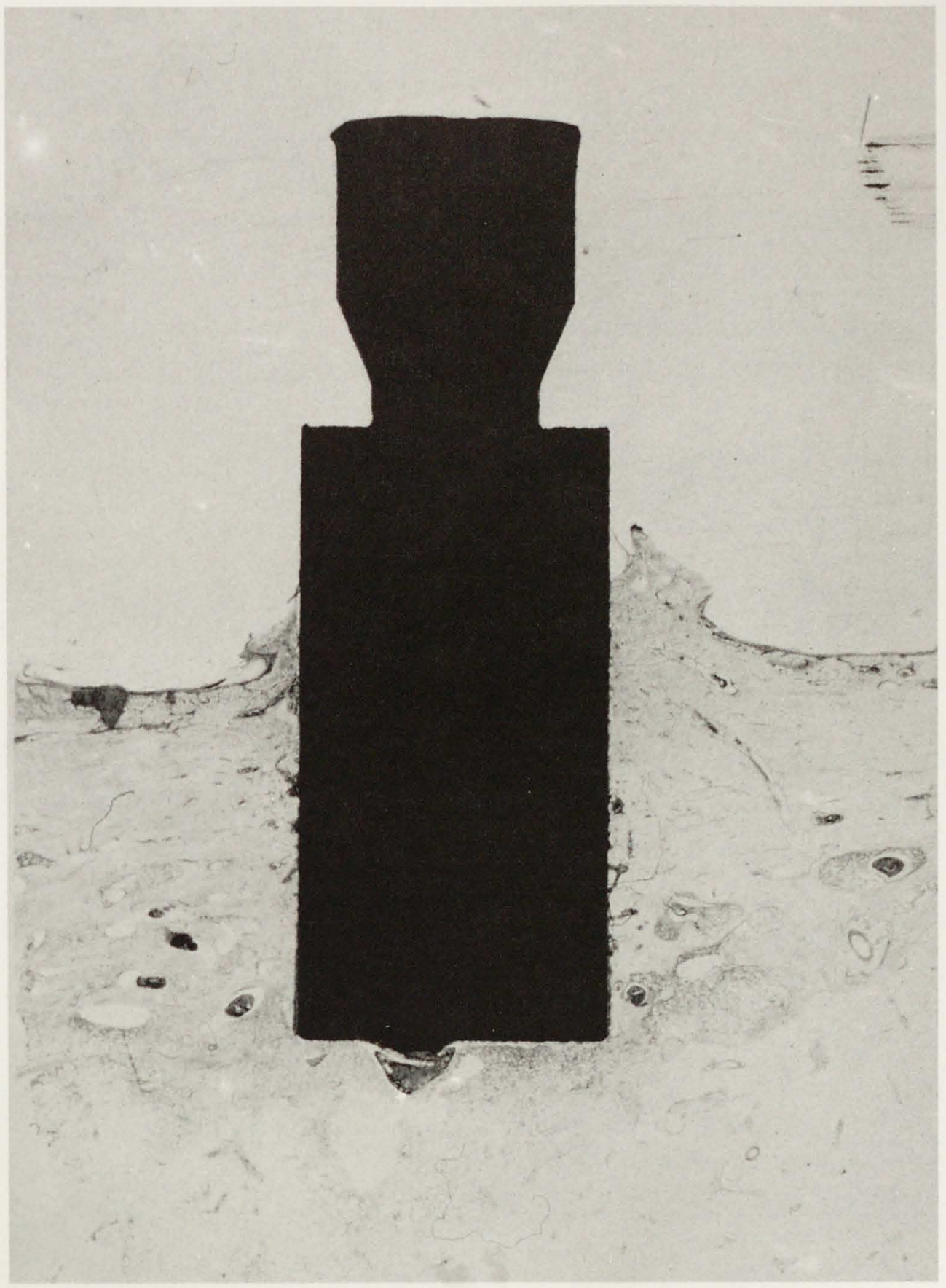

Fig. 24

Bone proliferation around hydroxyl-apatite coated plug. (basic fuchsine, six weeks, xl). 


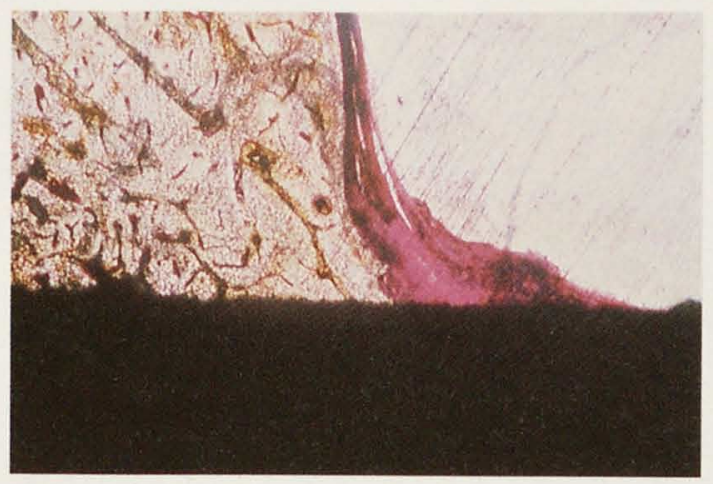

Fig. 25

Proliferation of peri-osteal tissue against hydroxyl-apatite coated plug. (basic fuchsine, six weeks, x5).

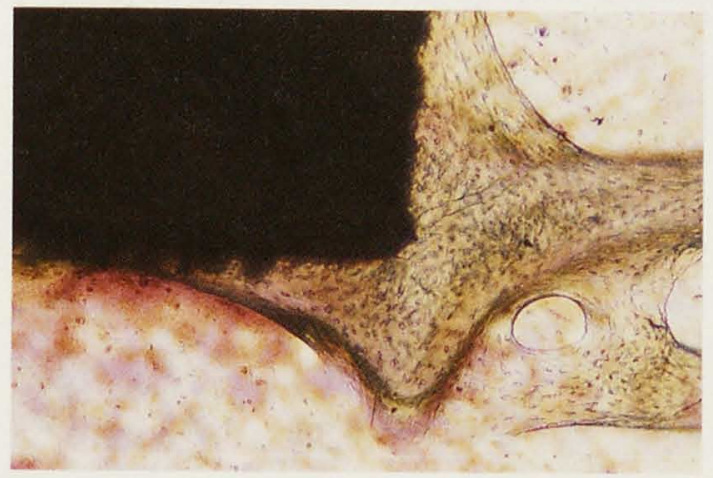

Fig. 26

Proliferation of endosteal bone against hydroxyl-apatite coating. (basic fuchsine, three months, $\mathrm{x} 5$ ).

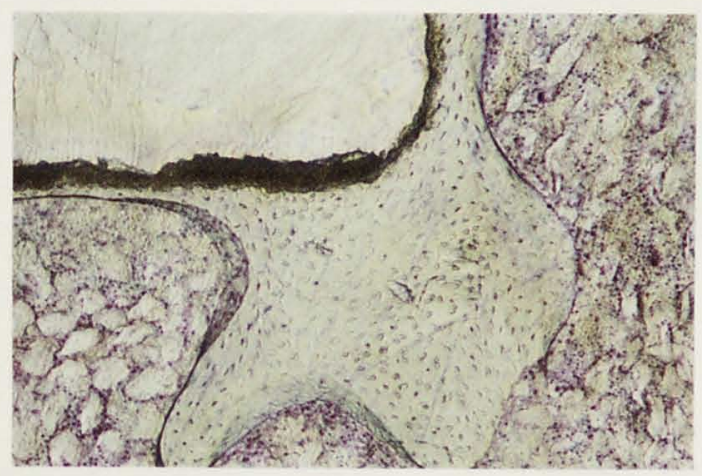

Fig. 27

Similar to previous, after removal of plug metal. The hydroxyl-apatite coating remains at the bone side of the interface. 


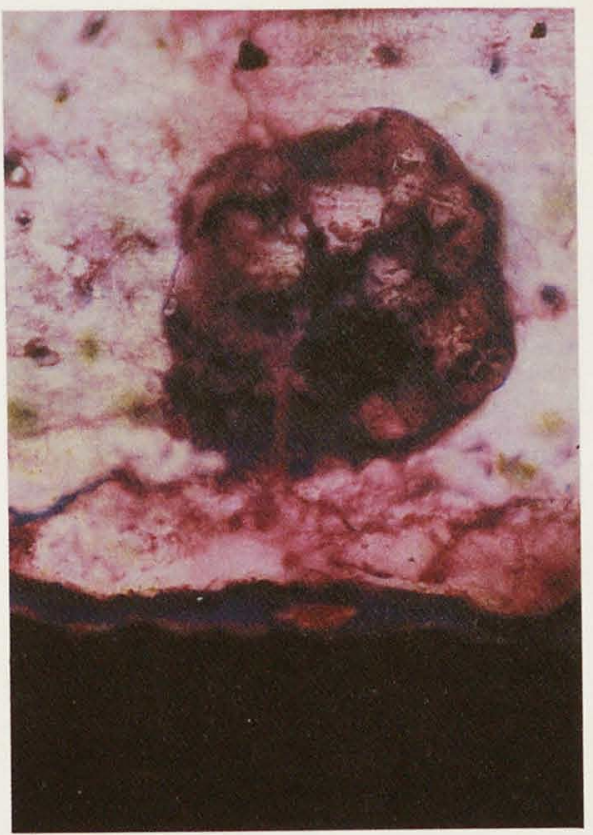

Fig. 28

Group of osteoblasts with deposition of osteoid material on hydroxyl-apatite coating. (basic fuchsine, three months, $\mathrm{x} 300$ ).

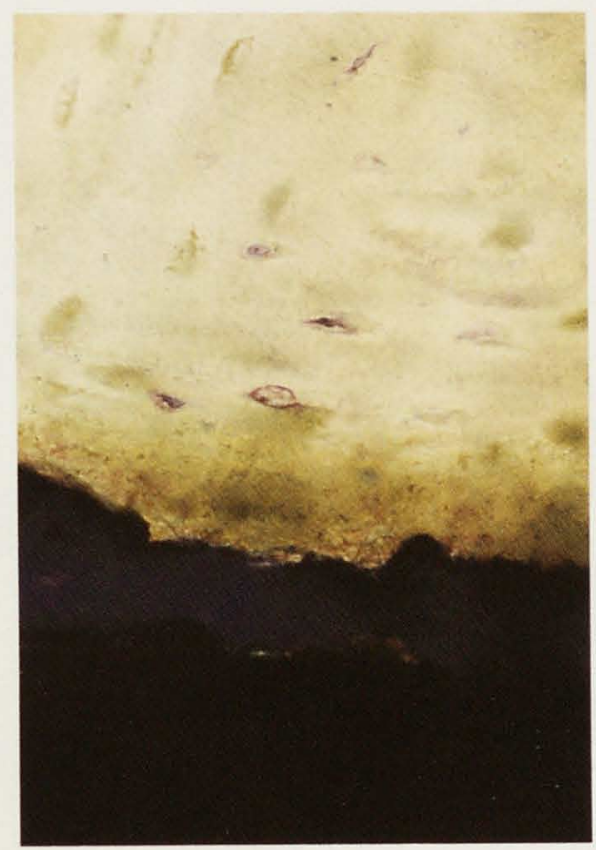

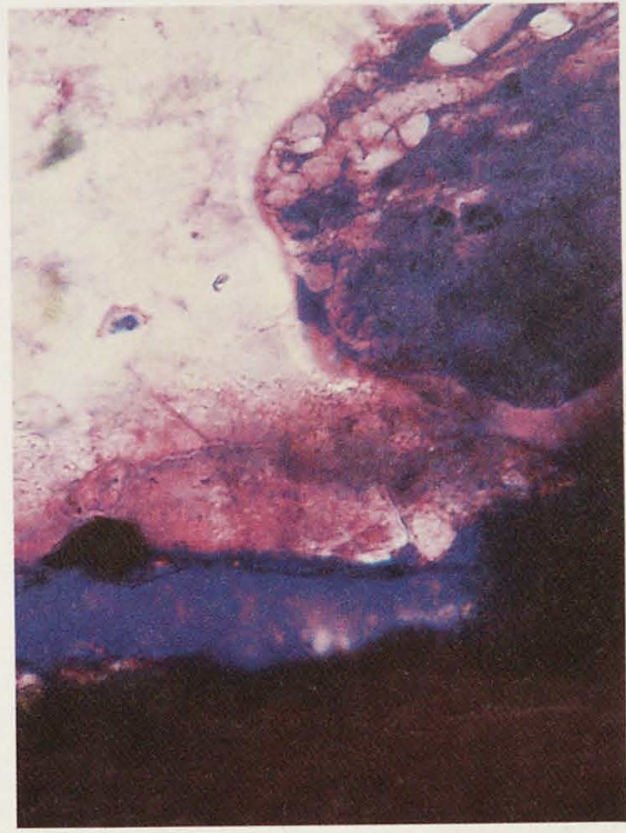

Fig. 29

Detail of osteoblasts, osteoid tissue and apatite (blue) coating-bone interface. (basic fuchsine, three months, $\mathrm{x} 600$ )

Fig. 30

Transition characteristics of bone towards hydroxylapatite coating. (basic fuchsine, three months, $\mathrm{x} 1000$ ). 


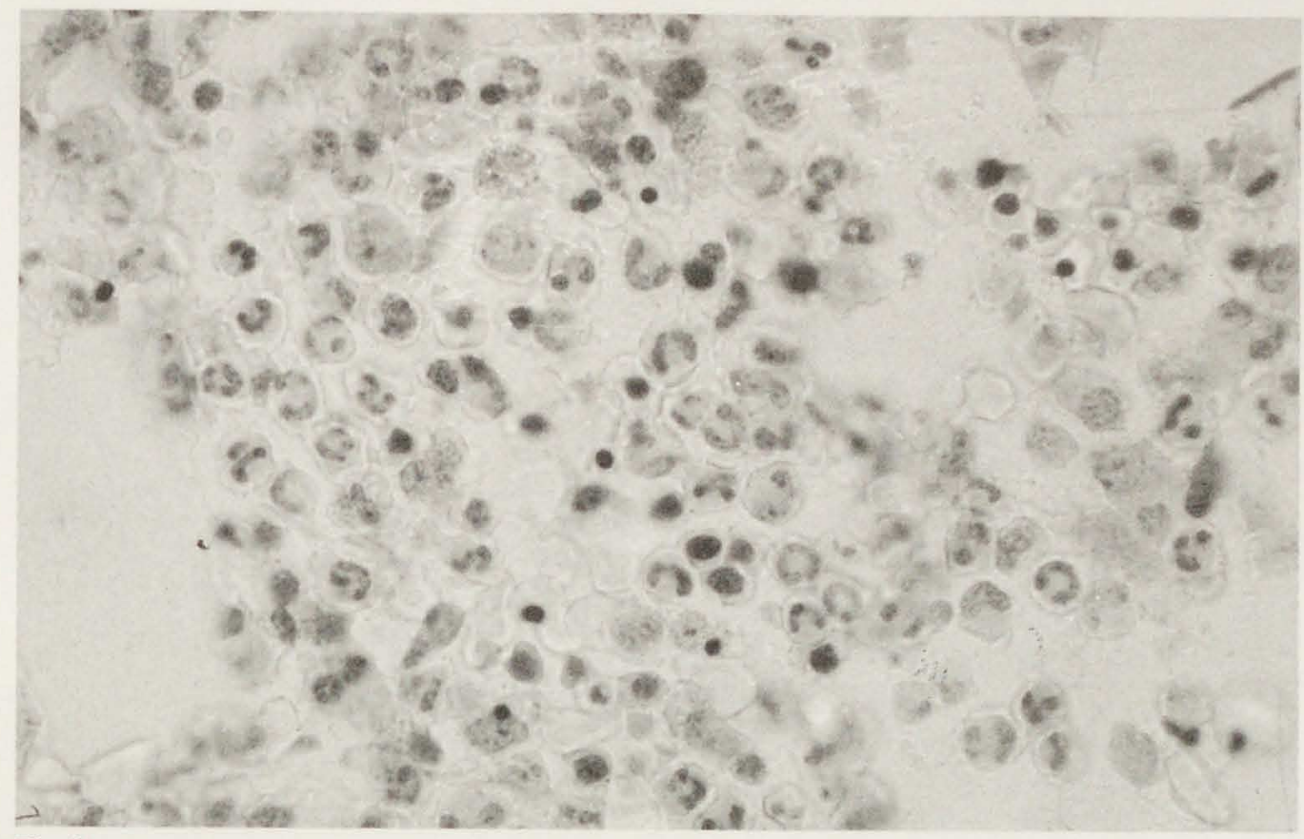

Fig. 31

Polymorpho-nuclear leucocytes in bone-marrow below hydroxyl-apatite coated plug implants. (HE, 6 weeks, $\mathrm{x} 600)$

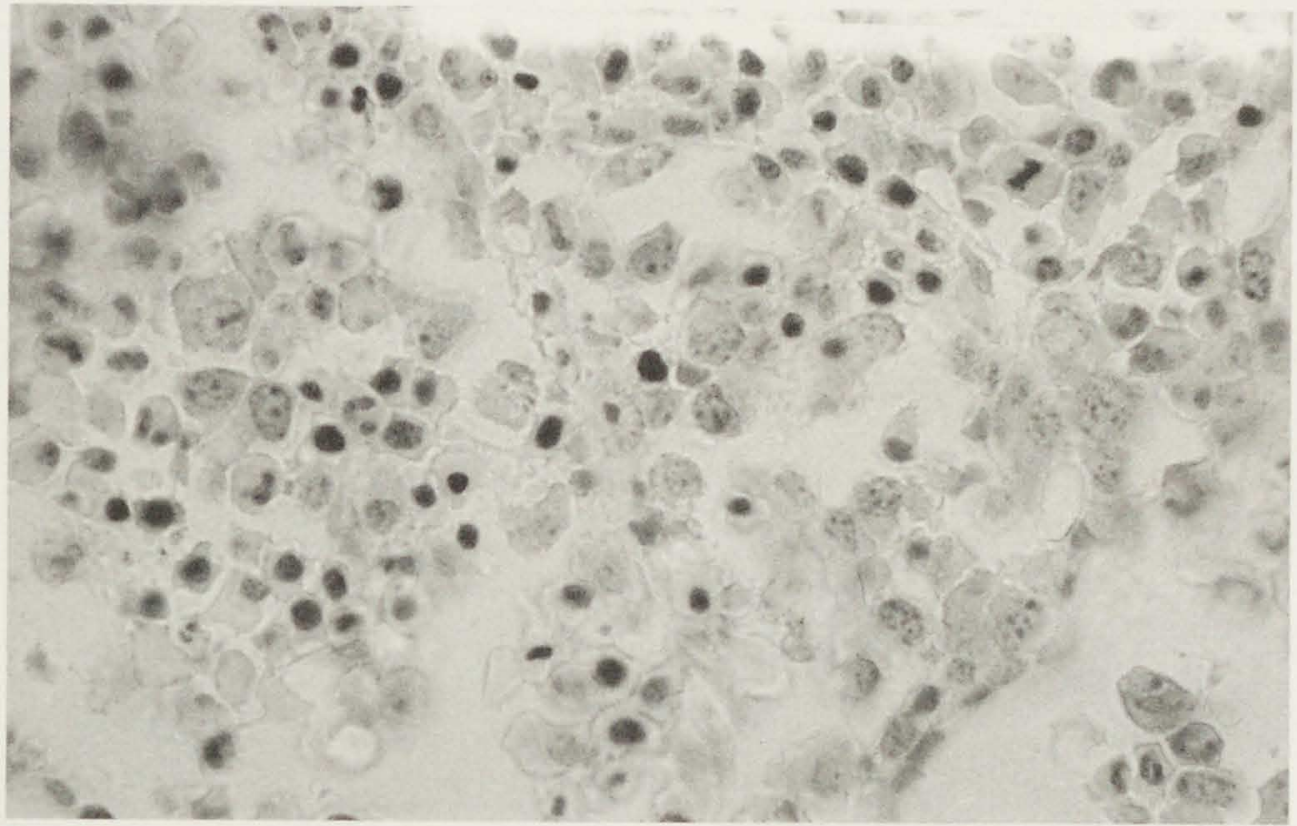

Fig. 32

Lymphocytes and plasma-cells in bone-marrow below hydroxyl-apatite coating (HE, 6 months, x600) 
Although dense bone spreads from the cortical area towards the endosteal protruding part of the plug, there is only a thin layer of bone at the most axi-central part of the plug. The increase in thickness of cortical bone, in contact with the plug, was on the average two millimeters at both endosteal and periosteal side of the implant.

In the first few months after implantation there is some inflammatory response of the bone-marrow. There are many polymorpho-nuclear leukocytes (Fig. 31). After three months follow-up this inflammatory response ceases and the bone marrow gets a more normal appearance. Mainly lymphocytes and plasma-cells are present, with scarcely some polymorpho-nuclear leukocytes (Fig. 32). These pictures are comparable with the normal histology of canine bone-marrow (Andersen 1970).

\section{Condition of hydroxyl-apatite coating}

In the microscopical sections after one and two years, the hydroxyl-apatite coating is still discernible as such. It has a smooth and dense appearance with a sharp transition from remaining coating to bone (Fig. 33). There is no fragmentation of ceramic particles and there are no persisting surface irregularities. The thickness of the coating can be determined with the aid of a micrometer. A remaining thickness of 10-30 microns can regularly be observed. The recorded values are however not very precise, because there is no guarantee of the measurements being made perpendicular the implant surface. Also, initial coating thickness was somewhat variable and not precisely known for each location. Some degradation of the hydroxyl-apatite coating is thus certainly possible, as will be discussed later on. There were however no areas with complete loss of hydroxyl-apatite coating on any plug.

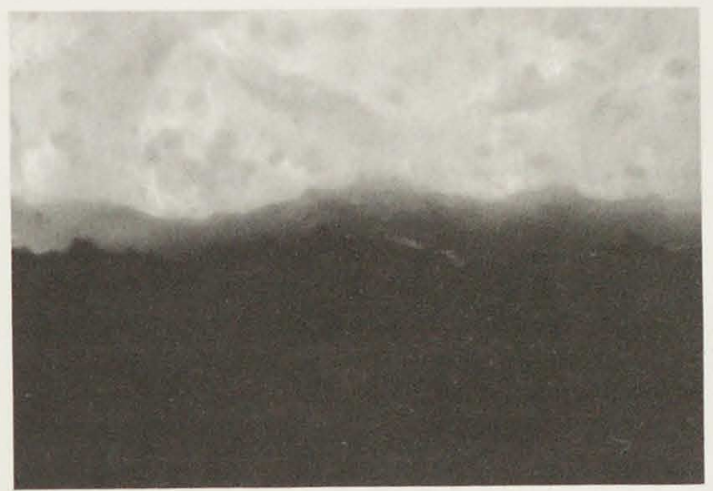

Fig. 33

Structure of hydroxyl-apatite coating after one year (polarization, $\mathrm{x} 200$ )

\section{Microradiography}

Microradiography of microscopical sections gives insight into the extent of mineralization of newly formed bone. In the early postoperative interval, six weeks, there is already abundant calcification in the transition area between implant with hydroxyl-apatite coating and bone (Fig. 34). The newly formed bone has a woven and lamellar structure. After three months and longer, the bone becomes much denser. It assumes the 
characteristics of dense cortical bone. There is an increase in radio-density of bone towards the implant with hydroxyl-apatite coating (Fig. 35). This means there is an increase in calcium content of bone in close contact with the coating. This area corresponds with the area of darkening of bone, as seen in the light-microscopical sections.

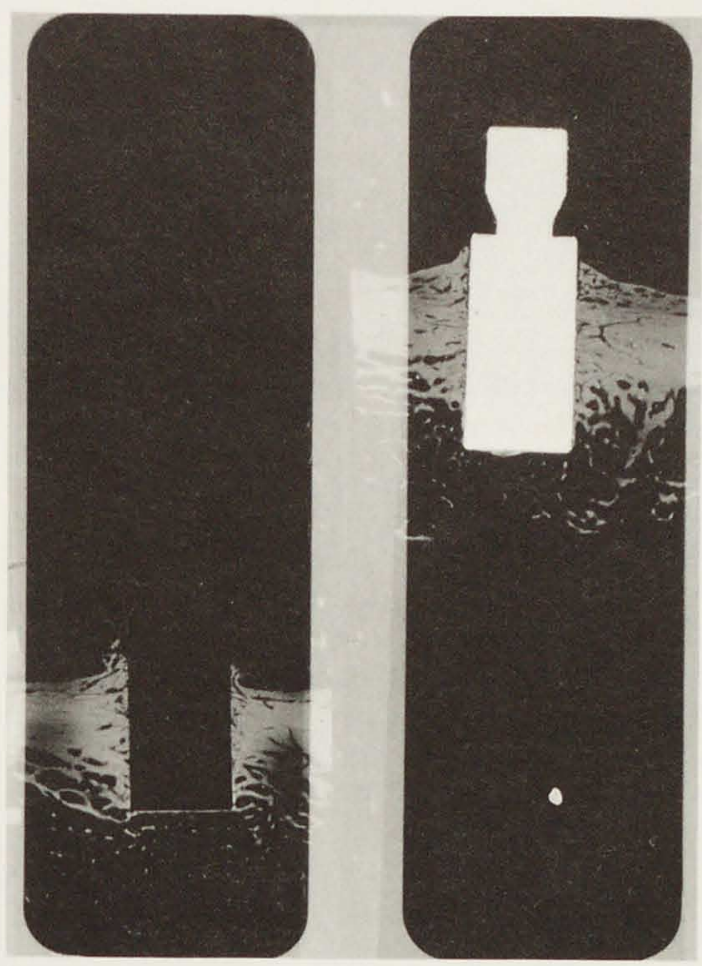

Fig. 34

Micro-radiograph of transcortical plug implant (6 weeks, $\mathrm{x} 1$ ) with and without plug metal.

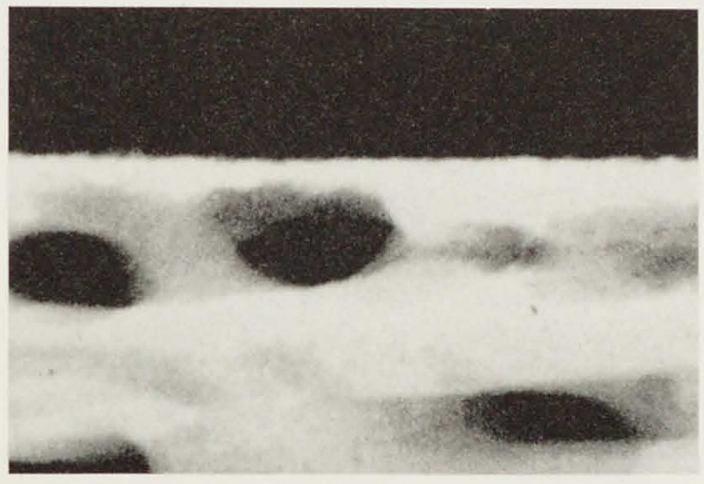

Fig. 35

Mineralization of new bone trabeculae at coating-bone interface. (micro-radiograph, 6 weeks, $\mathrm{x} 50$ ) 
Scanning-electron analysis of implant-bone interface

On a few sections electron probing was performed in order to establish the $\mathrm{Ca} / \mathrm{P}$ ratio of the bone in contact with the coating (Fig. 36). The obtained values indicate a $\mathrm{Ca} / \mathrm{P}$ ratio of near $10 / 6$. This means that the bone in contact with the hydroxyl-apatite coating has a normal composition and calcification.

\section{CA/P RATIO BONE APATITE COATING}

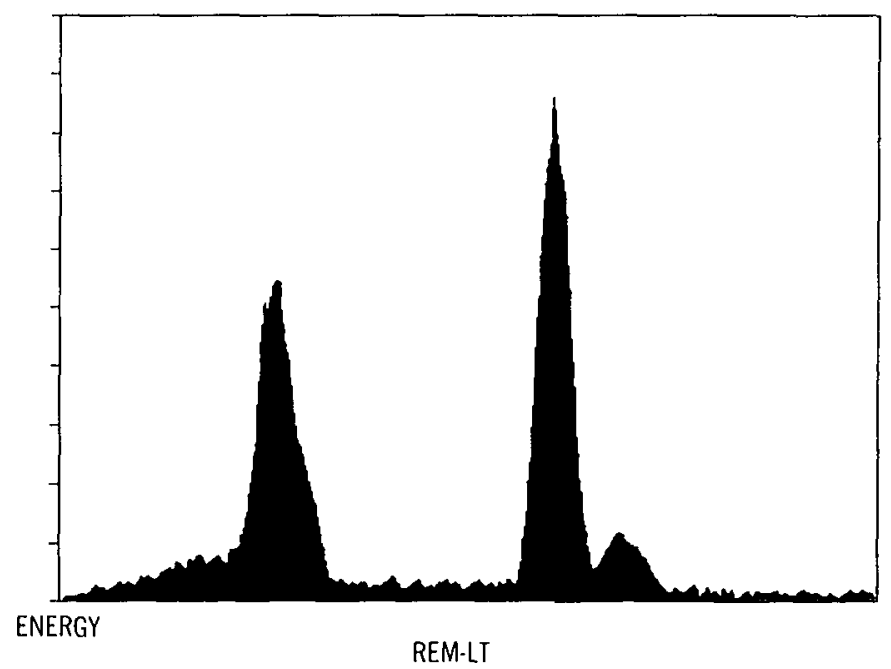

Fig. 36

Electron back-scatter analysis of bone composition at coating-bone interfacc. Ca/P ratio of $10 / 6$.

\section{Discussion}

The average plug-bone push-out force was 1440 Newton after the third month follow-up interval. Because the plugs were initially completely loose, with a push-out force of zero, the occurrence of plug-bone bonding to this extent is indeed significant. The mean plugbone interface shear strength with the first series of hydroxyl-apatite coated implants was $59 \mathrm{MPa}$. Based on paired T-test calculations, there proves to be no statistical difference at the $p<0.05$ significance level between values of shear strength after the three month or one and two year interval. Although the numbers indicate, there is good bone bonding starting early after surgery, they are probably too small, to provide hard proof of any statistical differences. On fresh bone, an average push-out force of $720 \mathrm{Newton}$ and a plugbone interface shear strength of 29.7 MPa was obtained after two years. Values for plugbone interface shear-strength, measured directly after sacrifice, proved thus to be approximately one half as compared to those obtained somewhat longer after sacrifice, where time delay and/or preparation artifacts of formalin can give distortion of results. Although the values obtained per dog showed rather small deviations in shear strength rates, the inter-animal variation was much higher. The figures probably indicate there is a rather consistent process of bone-bonding going on, the ultimate limit of this however 
being determined by the quality of the bone of the individual animal. Especially the short time interval in which a hydroxyl-apatite coating can achieve a strong bone bond and the fact that close apposition between bone and coating at surgery is not necessary to achieve good results are striking.

The obtained values for plug-bone interface shear strength in vivo from this study are higher than the static shear strength of the hydroxyl-apatite coating in vitro, as determined in chapter III. In fresh specimens the in vitro and in vivo strengths are comparable. Using the average cortical wall thickness of $1.75 \mathrm{~mm}$., fresh shear strength values are $30 \mathrm{MPa}$. This is the level of the in vitro results and probably represents the most realistic value. It is probable that the higher shear strength values obtained in the first series of hydroxylapatite coated plugs are caused by delay in measurements, due to some shrinkage of the bone around the plugs by formalin, thereby increasing their frictional resistance against push-out. Because failure mode, even under these circumstances, usually was within the hydroxyl-apatite coating layer, the recorded values still reflect the shear strength of the coating. There are however still some differences in failure mode which can be explained as follows: in vitro, the hydroxyl-apatite coated testing objects, are glued to the test housing with the aid of epoxy-resins. These glues only fix the surface layer of the hydroxylapatite coating. As the superficial layer of an hydroxyl-apatite coating is somewhat porous, the physical strength of this superficial 10-15 microns layer will be lower than its more deeper layers. Failure during this type of testing predominantly occurred at the epoxyapatite interface. With biological bonding of the hydroxyl-apatite coating in vivo this superficial 10-15 microns thick porous layer will be resorbed in the first few months and replaced with bone. Ultimately, only the deep dense part of the hydroxyl-apatite coating remains and becomes bonded to the bone. The physical strength of this layer is higher and its bonding power to bone proves higher than that to epoxy-resins. Shear tests under these conditions more regularly fail at the metal or within the hydroxyl-apatite material.

The histological results do not precisely indicate, how much resorption there has been with time. They just indicate that the coating is still there. The initial thickness of the hydroxyl-apatite coating was somewhat variable, especially in the early years of preparation of hydroxyl-apatite coatings. Furthermore, non-destructive pre-implantation measurements of coating thickness are rather inaccurate. Based on electronic measurement of the capacity between test-electrode and target-substrate, they measure the maximum coating thickness, over the "tops" of the microscopic "hills" of the coating. Any excessive local "peak" irregularities in coating thickness are more prone to dissolution. Additionally, microscopic sections need not be perpendicular to the substrate metal and may thereby cause distortions in recorded values and give false positive impressions of loss of coating thickness. Some degradation of the hydroxyl-apatite coating can therefore not be excluded, and has probably indeed occurred. It is known that in the first few months after implantation, during acquisition of bone-bonding, some superficial degradation of the hydroxyl-apatite coating occurs, because of the increased surface porosity of the outer layer of the coating. After two years follow-up, the remaining coating thickness appears more than adequate to insure implant fixation. Remaining coating thickness in itself is not very critical, because bonding to hydroxyl-apatite coatings is a surface phenomenon and not dependant upon any depth of ingrowth into the coating that 
has a closed surface and dense structure of its deep layer. Longer follow-up than current two years are necessary to establish the long term stability of hydroxyl-apatite coatings.

The mechanism of implant-bone bonding is thought to be chemical and biological. Evidence supporting the chemical bonding concept comes from the histological results. Numerous osteoblasts are in direct contact with the hydroxyl-apatite coating without any interposition of fibrous tissue. The osteoblasts deposit their osteoid directly on the coating. The bone quality in the coating-bone transition area is very good and there is an increase in calcium content of bone towards the coating.

Biocompatibility as measured in the bone-marrow is good. Initially there are many polymorpho-nuclear leukocytes. This is probably caused by the surgical trauma of implantation. With three months and longer follow-up, the bone-marrow reassumes its usual appearance, consisting of a mixed population of lymphocytes, plasma-cells and a few other cell lines.

In contrast to the coated implants, non-coated titanium implants do not show any bonebonding properties (Ronningen ea. 1984). The highest value obtained for interface shear strength was $0.6 \mathrm{MPa}$. The rate of improvement in bone bonding by hydroxyl-apatite coatings is thus almost fifty fold. Their figures indicate that the principle of "osseointegration" of implants is in fact no more than a synonym for bio-inertness of implant materials. The fixation of such implants to bone must, by lack of any adherence to bone, rely on principles of mechanical retention.

Comparison with literature data on cemented and non-cemented plug implants of comparable design is difficult and questionable because of differences in experimental setup and interpretation of results. Under laboratory conditions, the implant-bone interface shear strength for acrylic bone cement lies between 5,6 and 20,7 MPa, as reported in the literature (Lange 1979, Halawa ea. 1978). In vivo interface shear strengths range between 2,4 and 5,7 $\mathrm{MPa}$ (Krause ea. 1982). They strongly depend on the quality of preparation of the cement-bed and the possibilities for cement penetration in bone by pressurization.

Using porous-surfaced metallic implants, reported interface shear strengths range between 17 and $25 \mathrm{MPa}$ (Cook ea. 1985, Anderson 1984, Pilliar ea. 1983, Bobyn ea. 1980). The higher values were obtained with multi-layer coatings and/or surface porosities of up to $50 \%$. Both are factors, known to increase the susceptibility for fatigue failure of such porous metallic coatings (Manley ea. 1987). Theoretically, and from the literature, a limit of $20 \mathrm{MPa}$ for interface shear strength is realistic for porous surfaced metallic implants. Although the strength rates obtained with hydroxyl-apatite coatings are not much higher, as compared to porous-metal coatings, especially the speed with which a hydroxyl-apatite coating can achieve a strong bone bond and the fact that close apposition between bone and coating at surgery is not necessary to achieve these results is quite different from a porous-metal coating.

Using hydroxyl-apatite coatings some authors have obtained lower shear strength values (Manley ea. 1987, Kay ea. 1986). Results are very dependent upon experimental set-up. Kay's implants all failed outside the apatite-bone interface within the bone itself and hence 
refer to a different interface mode failure than with our experiments. Experiments were carried out with hydroxyl-apatite coated intra-medullary nails in experimental osteotomies. Because the mechanical strength of trabecular bone varies widely (approximately 4-70 MPa) and is invariably lower than that of cortical bone, this strongly affects the maximum obtainable interface strength. The quality of the bone is then the limiting factor and not the properties of the hydroxyl-apatite coating itself. Even then, it can not explain why failure in their experiments occurred in the bony area around the plug. If failure mode is within the bone, then the hydroxyl-apatite coating, including its bony interface, must be stronger then the surrounding bone. Their data are therefore in conflict with literature data on mechanical strength of cortical and trabecular bone and are probably on the conservative side.

In this study, we obtained a mean interface shear strength of approximately $30 \mathrm{MPa}$ by means of chemical bonding between implant and bone using hydroxyl-apatite coatings and bone. Although the absolute magnitude of the obtained plug-bone interface strength rates is subject to interpretation errors, the relative increase in plug-bone bonding from zero to the obtained high values is certainly significant. Especially the speed with which a hydroxyl-apatite coating can achieve a strong bone bond and the fact that close apposition between bone and coating at surgery is not necessary to achieve these results is striking. Although the results of this experimental study can not be fully extrapolated to the much more complex human situation, the fundamental principles involved in the concept of chemical bone-bonding are of high clinical relevance. The results indicate that with hydroxyl-apatite coated implants an implant-bone bonding can be achieved of high strength. Although this is well known for sintered hydroxyl-apatite implants, the elimination of fatigue failure by using hydroxyl-apatite coatings on metal substrates is a substantial improvement. The conclusion of this plug implant study is, that hydroxylapatite coatings have a similar biological response as compared to sintered hydroxylapatite implants. Their physical strength including fatigue strength is much better and their biocompatibility and biostability appear the same as those of sintered hydroxylapatite. 


\section{Chapter seven}

\section{Canine hydroxyl-apatite-coated total hip replacement}

Strong bonding properties between hydroxyl-apatite coated plug implants and bone have been established in the previous chapter. A shortcoming in the plug study was that the plugs were not mechanically loaded during the time of acquiring bony fixation. According to literature reports, there can be a difference in performance of the same biomaterial under loaded or unloaded conditions (Heck ea. 1986, Griss ea. 1978 \& 1977). Under mechanical loading, micro-motion inevitably occurs between implant and bone if there is no strong fixation between the two. The difference in the modulus of elasticity between implant material and bone as well as their differences in cross-sectional geometry are important causal factors for this micro-motion. Micro-motion at the implant-bone interface impairs the transition of capillaries across the interface and thereby development of bony fixation (Perren 1983). A fibrous tissue interface is then the end result under conditions of load-bearing, in stead of solid bony encapsulation under conditions of mechanical rest. This is especially true for bioinert implant materials. The mechanical loading limits for this fibrous "pseud-arthrosis" tissue are much lower than for cortical bone. Although some authors (Walker ea. 1984) suggest that such a fibrous tissue layer can be effective in distributing interface stresses towards the surrounding bone, we must realize that the mechanical loading limits for fibrous tissue are so low, that fibrous tissue cannot be expected to bear any significant interface stresses in any physiological way. Additionally, the thickness of fibrous tissue membranes around cementless implants appears progressive with loading and movement of the implant (Hedley ea. 1982), thereby increasing (micro)-motion of the implant in a vicious circle. For these reasons, "pressfit" implantation using a soft fibrous tissue interface is prone to failure.

To further investigate the mechanical and biological characteristics of hydroxyl-apatite coatings under mechanical loading a canine total hip replacement study was initiated. Results of hydroxyl-apatite coated hip prostheses were compared with otherwise similar non-coated controls, both having the same pressfit fixation in bone. Evaluation was made with the use of mechanical, radiological, scintigraphic and histological techniques. The objective of the study was to determine whether hydroxyl-apatite coated implants provide better biological as well as mechanical characteristics of implant fixation, as compared to non-coated bioinert titanium implants, under conditions of heavy joint-loading. If hydroxyl-apatite coated implants should prove to have effective bonding properties to bone even under conditions of early heavy weight-bearing, then human total hip replacement using hydroxyl-apatite coated implants becomes feasible. 


\section{Materials and methods}

This section on materials and methods is divided into the following paragraphs:

design characteristics of canine total hip prosthesis

surgical technique of canine total hip replacement

follow-up protocol of dogs

mechanical evaluation of implants

radiological evaluation of implants

scintigraphic evaluation of implants

histological evaluation of implants

\section{Canine total hip prosthesis}

The most current veterinary hip prostheses use acrylic cement for bone fixation, by design they are therefore less useful for cementless implantation using hydroxyl-apatite coatings (Olmstead ea. 1983 \& 1981). To optimize prosthetic parameters for chemical fixation to bone, we decided to develop our own canine total hip prosthesis.

The canine total hip prosthesis was designed with the following required characteristics:

- titanium substrate

- anatomic adaptation to proximal femur of dog

- size adapted to dogs of initially $30-35 \mathrm{~kg}$

- textured surface on one side of prosthesis

- hydroxyl-apatite coating on femoral stem

- cement fixation of acetabular component

- head diameter of $18 \mathrm{~mm}$.

- accurate instrumentation

Titanium is used for reasons discussed earlier (chapter I).

Additionally, the non-coated control implant has the characteristics of titanium, to provide it the best possible biological performance available in its class of bioinert materials. Although there might be differences in design criteria for implants intended for rigid bony fixation (hydroxyl-apatite) as compared to non-rigid "pressfit" fixation, the same implant geometry was chosen for both coated and non-coated implants. This was done in order to study exclusively the effect of the hydroxyl-apatite coating on bone bonding and bony integration.

To determine the geometry of the proximal femur 15 dog femora were radiographed and digitised. The typical hip of a dog has a much shorter femoral neck as compared to its human counterpart. Also, the acetabulum is often somewhat dysplastic. A custom series of implants was manufactured by Stryker ${ }^{\circledR}$ according to the data obtained (Fig. 37). One side of the prosthesis was textured to study any differences between smooth and stepped surfaces. Because of the small size of dog acetabula, a metal screw ring with polyethylene insert would result in too small a head diameter. Therefore, a conventionally cemented acetabular component was used with an internal diameter of $18 \mathrm{~mm}$. and an external diameter of $26 \mathrm{~mm}$. To limit the number of prosthetic sizes the dogs (german shepherds) 


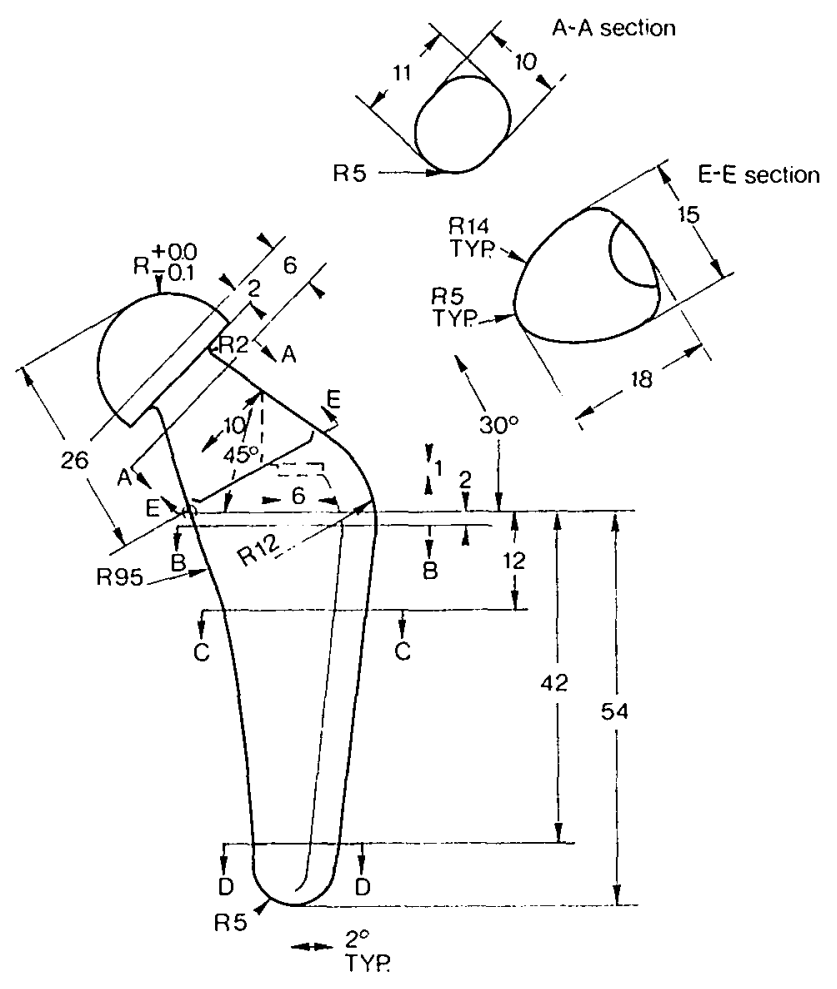

Fig. 37

Geometrical characteristics of canine total hip implant.

were selected in the weight-class of approximately $30 \mathrm{~kg}$. Preoperative radiography served proper sizing of the implant to the dog.

To maintain a reproducible technique of implantation, the instruments were also specifically designed for the purpose. They consist of conical intra-medullary reamers in three incremental sizes to effect the reaming of the axi-symmetric part of the implant (Fig. 38). The inclination angle of the conical reamers is three degrees. The corresponding calcar rasps effect the preparation of the asymmetric calcar part of the implant bed.

Fifteen prostheses were prepared with a hydroxyl-apatite coating, using the plasma-spray technique and coating characteristics described in the previous chapters (Fig. 39). An additional number of hydroxyl-apatite coated samples served the purposes of coating quality control. Another fifteen similar prostheses were left uncoated to serve as controls. 


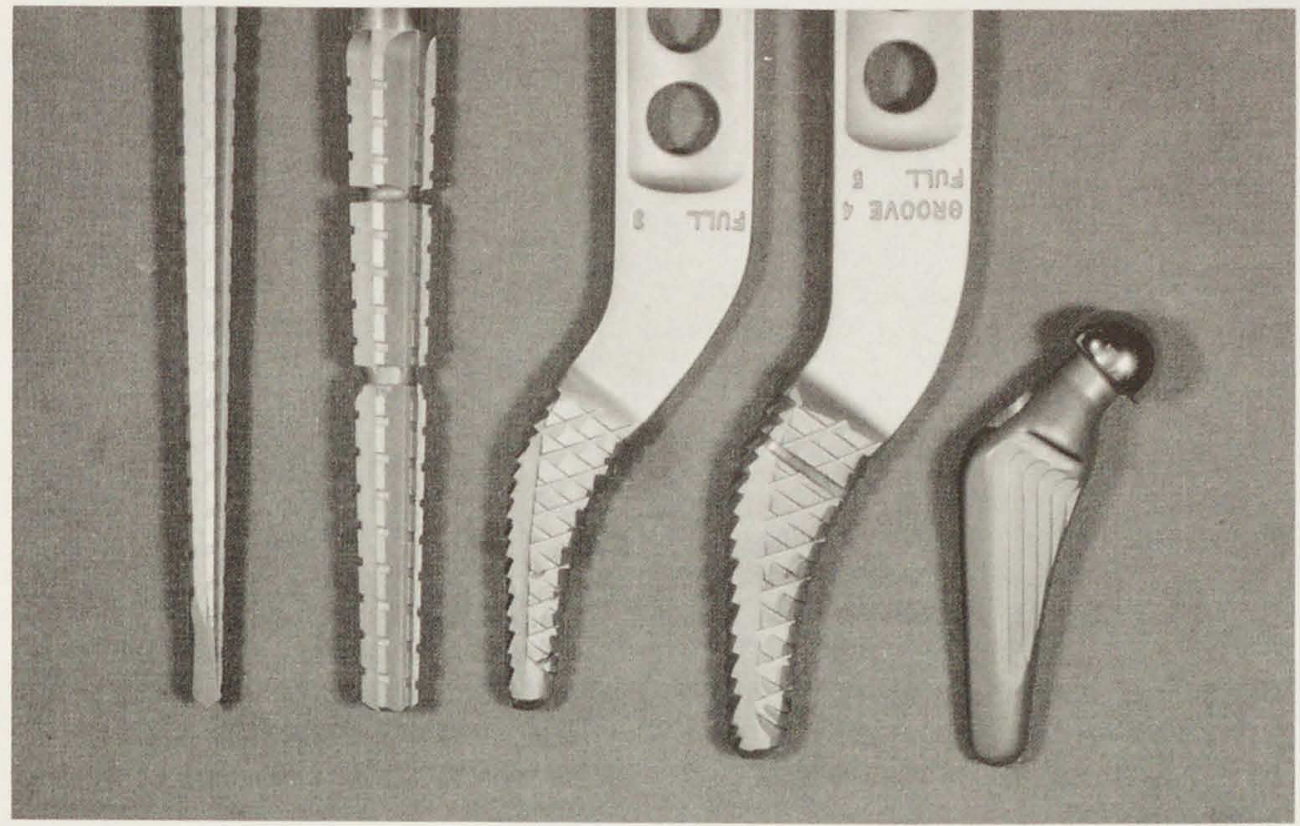

Fig. 38

Instrumentation and trial prosthesis for canine total hip replacement.

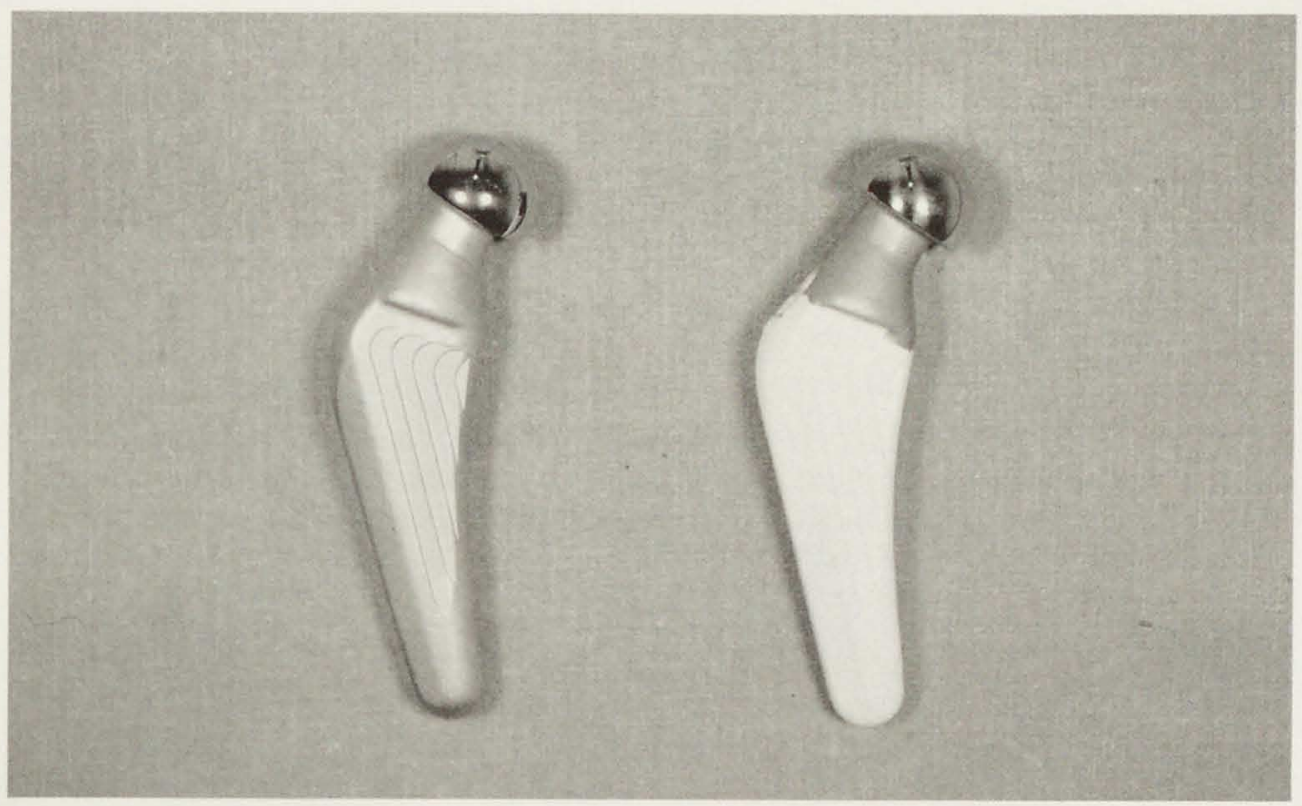

Fig. 39

Hydroxyl-apatite coated and non-coated canine total hip implant. 


\section{Technique of canine total hip replacement}

Fifteen animals were operated using the following techniques of anaesthesia:

- general anaesthesia

- intubation and controlled ventilation

- intravenous lines

- cardio-pulmonary monitoring

- induction of anaesthesia with penthotal ${ }^{\circledR}$

- maintenance of anaesthesia with fluothane ${ }^{\circledR}$

Antibiotic prophylaxis with cephradine $\left.{ }^{(}\right)$for 2 days (van den Boogaard ea. 1985). Fluorescence markers Tetracycline and Calcein according to alternating schedule.

\section{Protocol of surgical technique:}

The operative technique for canine total hip replacement conforms to current veterinary standards (Chen ea. 1983, Olmstead ea. 1983 \& 1981):

- side position

- free draping of operated leg

- postero-lateral approach

- H-shape capsular incision

- luxation of femoral head

- head/neck resection with aid of template

- preparation of proximal femur

- conical intramedullary reamers

- calcar rasps until correct trial fit

- preparation of acetabulum

- acetabular cartilage reaming

- 26 and/or $29 \mathrm{~mm}$ spherical reamers

- three retention holes for cement fixation

- trial reduction of both components

- bone cement fixation of acetabular component

- pressfit fixation of femoral component in femur

- reduction of hip prosthesis

- closure of synovial capsule

- wound closure

A hydroxyl-apatite coated prosthesis was implanted in the right hip and an otherwise similar non-coated prosthesis in the left hip (Fig. 40 and 41 ). The first hip was selected at random and the time interval between first and second operation was at least four weeks. A shorter time interval between first and second operation caused some postoperative dislocations of prostheses. 


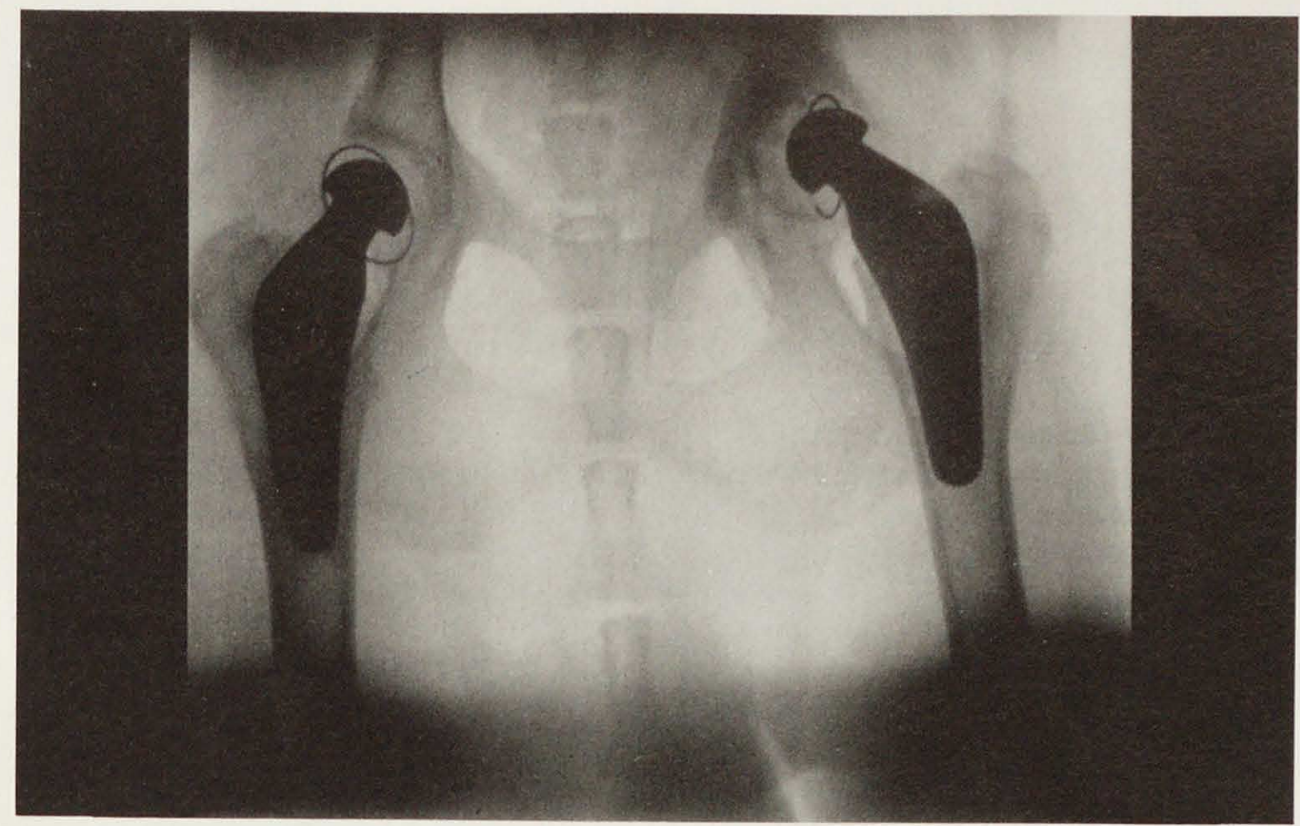

Fig. 40

AP radiograph of bilateral canine total hip replacement ( 1 year).

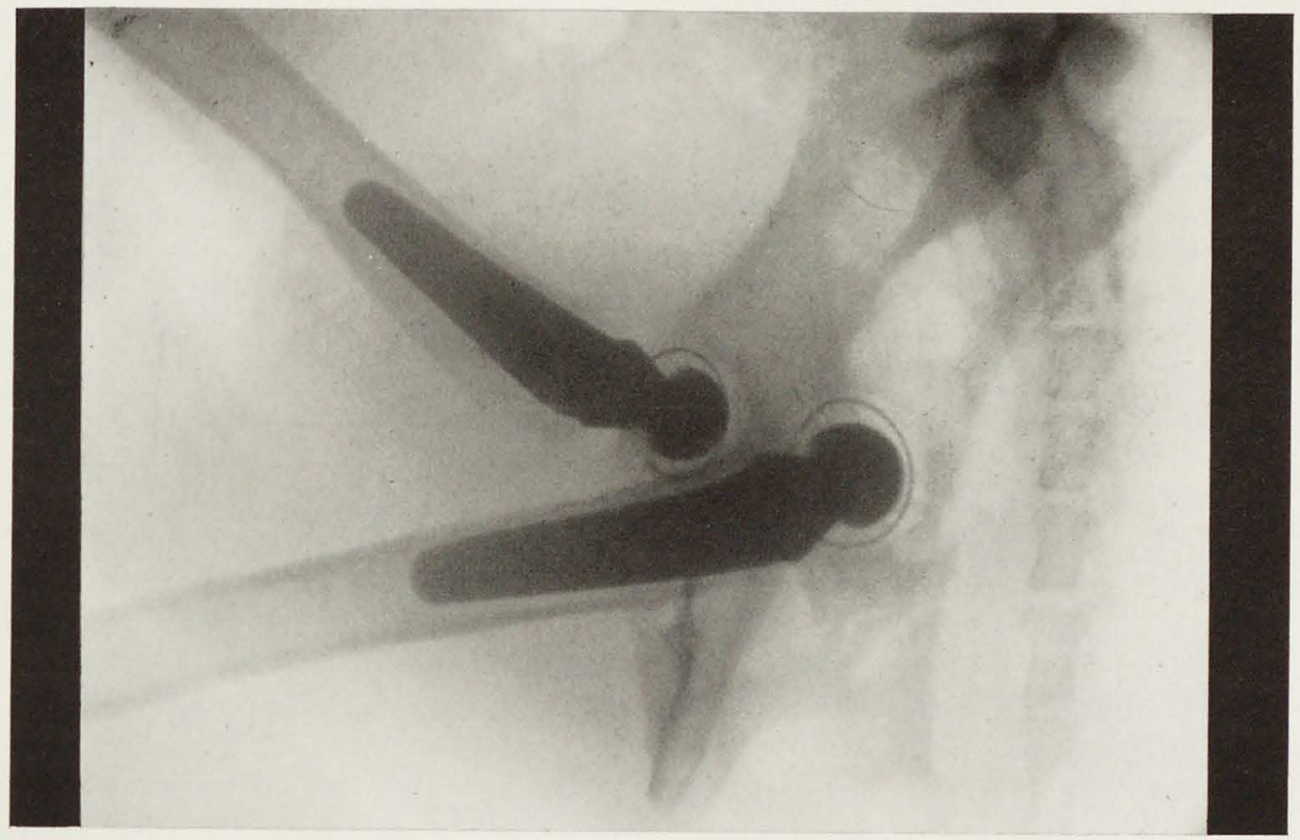

Fig. 41

Axial radiograph of bilateral canine total hip replacement ( 1 year). 


\section{Surgical complications}

Surgical complications occurred with four dogs. Two dogs had postoperative dislocations within the first few weeks that needed open reduction (Fig. 42). One of these acquired a deep infection, manifested by a fistula from the 12 th week. This dog (coated prosthesis) was excluded from the series, but will be discussed separately. One dog had several luxations within the first few weeks after implantation that were reduced by closed manipulation. Several dislocations related to an initial short time interval between first and second operation. After these incidents, the time interval between first and second operation was increased to at least four weeks.

One dog (non-coated prosthesis) sustained a spiral fracture of the proximal femur that needed cerclage wiring two days after hip replacement (Fig. 43). Further course of this dog was uneventful.

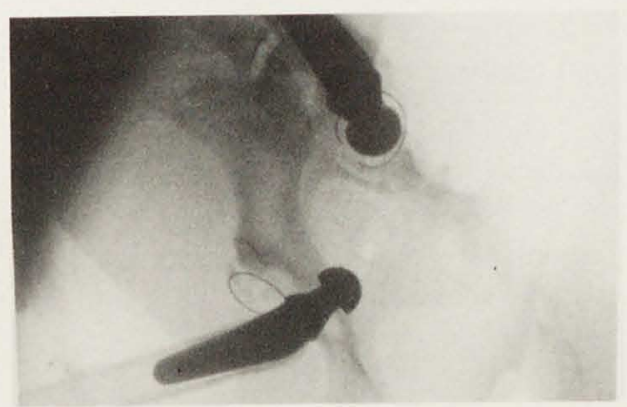

Fig. 42

Postoperative dislocation of canine total hip implant.

\section{Follow-up protocol}

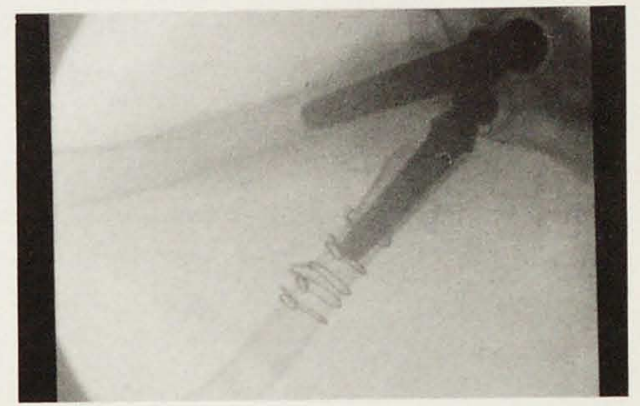

Fig. 43

Osteosynthesis for femoral fracture after canine total hip replacement.

The animals were left free to ambulate and usually resumed walking within a few days. Spontaneous protected weight-bearing lasted some two weeks. Starting the eight week after the last operation the animals were exercised in running and jumping. Body weight of the dogs generally increased from an average $30-35 \mathrm{~kg}$ to an average $50 \mathrm{~kg}$. The functional behaviour of the dogs was recorded according to the following criteria:

- walking on three legs

- partial weightbearing on operated leg

- full weightbearing on operated leg

- running

- jumping

\section{Sacrifice protocol of dogs:}

Two dogs each were sacrificed at intervals of:

-3 weeks

- 6 weeks

- 3 months

-6 months 
- 1 year

-2 years

- 5 years (future report)

Excluding the dogs with complications and preserving some animals for longterm followup ( 5 years), leaves ten dogs with eighteen implants for current evaluation.

Mechanical evaluation of implant-bone interface

With implants of complex design, like hip implants, it is very difficult to precisely quantify the mechanical characteristics of the implant-bone interface. The plug study served that purpose. Therefore, in this study we limited the mechanical evaluation of the implant fixation to clinical judgement. Moreover, forceful extractions of implants from the femur would have damaged the implant-bone interface, thereby impairing histological evaluation. We tried to move or extract the implants from the femur by manual force. One hydroxyl-apatite coated implant with six weeks follow-up was extracted on the test bench with hammer and chisel to observe any unexpected events.

\section{Radiographic evaluation of implants}

Pre-operative as well as post-operative X-ray pictures were obtained, for control of size and correct positioning of implants. At the time of sacrifice, explant radiographs were obtained on high resolution films. Both hydroxyl-apatite coated and control implant were exposed at the same time on the same film, so as to make good comparisons on bone quality feasible. The radiographs were evaluated with the following objectives:

radiographic aspect of implant-bone interface

radiolucent line formation

radiodense line formation

extent of new bone formation

extent of bone resorption

adverse radiographic effects of hydroxyl-apatite coating

\section{Scintigraphic evaluation of implants}

$99 \mathrm{~m}$-Technetium bone scans were obtained on the dogs with follow-up periods of six months and longer. A single dose of 1 millicurie $99 \mathrm{~m}$-technetium per $15 \mathrm{~kg}$ bodyweight was administered three hours prior to sacrifice. Using a gàmma-camera scintigraphic pictures were obtained in AP and axial directions of explants.

\section{Histological processing of implants}

After the predetermined intervals the dogs were sacrificed using a lethal dose of thiopental $^{\circledR}$. The aortic vessels were cannulated and the extremities perfused with a heparinized salt solution, continued with $5 \%$ formaldehyde solution. The hip joints were freed of excess soft tissue and the femoral bones exarticulated. Biopsies were taken from the prosthetic neo-capsule and bone-marrow as well as explant radiographs and scintigrams obtained.

Additional formalin fixation and alcohol dehydration of explanted bones. Microscopic sections of 10-50 micron thickness were prepared of the hydroxyl-apatite coated implants using a diamond saw with cooling. To facilitate the histological processing of the 
prostheses, the prosthesis-containing part of the femur was first divided into four segmental pieces with an industrial saw and the center of the implant metal was drilled out. Non-coated implants were easily extracted from the femur. Many of these femora were processed without the prosthesis to 5 micron thickness sections. Transmission light, polarization and fluorescence microscopy were used together with micro-radiography. From each implant sections were taken from proximal-, mid- and distal-stem area. Staining with Masson-trichrome, Alcian blue, hematoxylin-eosin (HE) or basic fuchsine.

\section{Microradiographic evaluation of implants}

Microscopic transverse sections of implants were micro-radiographed using ultra-high definition films to obtain data on the calcification sequences around coated and non-coated implants.

\section{Histological evaluation of implants}

Histology was reviewed with the following focus of interest:

general evolution of implant-bone bonding process

character of implant-bone interface (bone/fibrous tissue)

amount of new bone formation

amount of bone-resorption

density and structure of bone

potential for filling bone defects

differences in response with cortical or trabecular bone

condition of hydroxyl-apatite coating

response of bone-marrow (biocompatibility)

response of prosthetic neo-capsule (biocompatibility)

\section{Results}

\section{Functional behaviour.}

There were no obvious differences in functional recovery after operation between hips with coated or non-coated implants. Full weight-bearing took some two weeks to occur. Starting the eight week after the last operation the animals were exercised in running and jumping, although many of the dogs resumed these activities spontaneously at an earlier time (Fig. 44). Until the third month after surgery, there often was relative unloading of the affected limb with fast running and jumping. Three months after implantation animal behaviour was rather indistinct from normal with most of the dogs. One dog exhibited relative unloading of an uncoated implant, especially with fast running. His acetabular component proved to be grossly loose.

\section{Radiographic evaluation of prostheses}

\section{General comments}

Directly after surgery, there are many gaps and spaces around cementless implants. As measured on some preliminary cadaver implantations, the distance between bone and implant can be as much as $1.5-2 \mathrm{~mm}$. Even with accurate instruments, surgical preparation proves to be inexact and the total initial implant-bone contact area can be as 


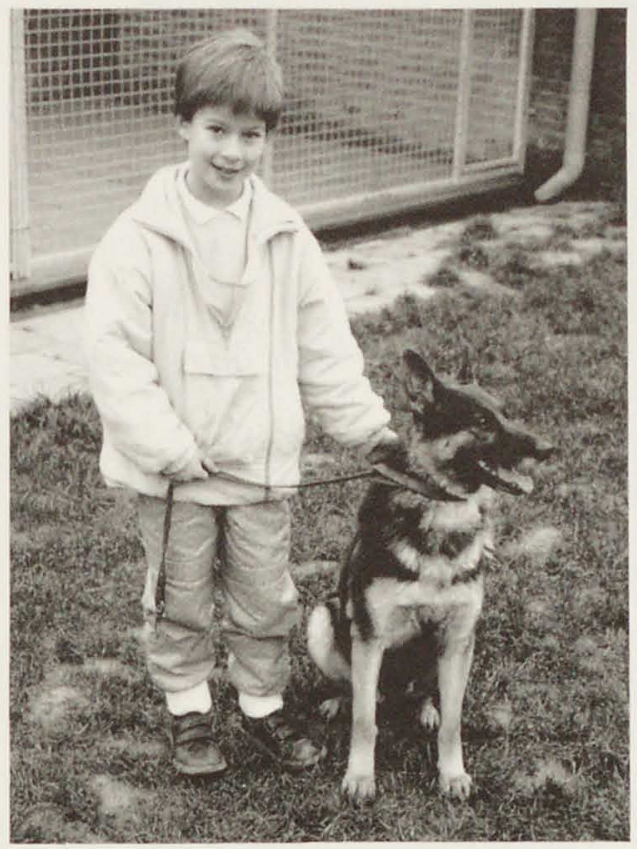

Fig. 44

Long term survivors.

small as ten percent and seldom exceeds twenty percent (Fig. 45). This is caused by the large variations in proximal femoral geometry, inaccuracy of intra-medullary reaming in clinical practice and the deformation of the femur during insertion of the much stiffer metallic implant.

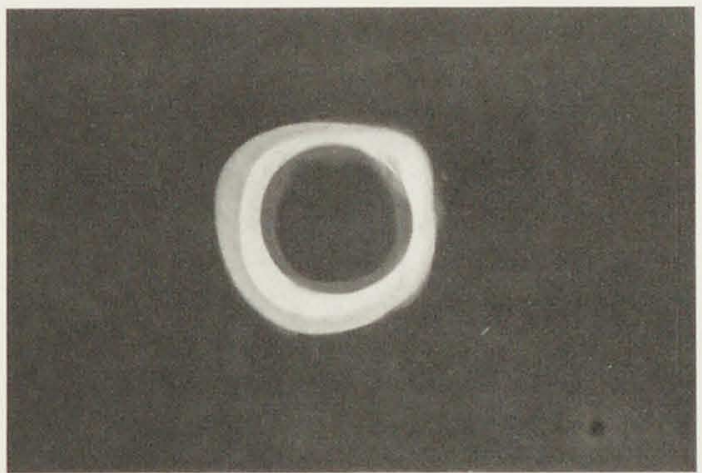

Fig. 45

Two point bone contact only in lateral trochanteric region. Empty space around implant circumference two weeks after implantation (pilot study). Observe periosteal thickening shortly after implantation. (Micro-radiograph of proximal stem area cross-section, $\mathrm{xl}$ ). 


\section{Radiographic evaluation of non-coated implants.}

The first three to six weeks after implantation of an uncoated hip implant, radiographic appearance is indistinct from a coated prosthesis. The bone defects caused by the intramedullary reaming during the time of implantation are clearly visible. From the third week on, until longest follow-up of two years there develops an increasing difference in bone quality between coated and non-coated prostheses (Fig. 46 and 47). From the sixth week on non-coated prostheses develop radio-lucent line formation. The initial thickness of these lines is about $0.2 \mathrm{~mm}$. around the entire femoral stem area. With time this line progresses still somewhat and after one to two years there is a radiolucent line of at least $0.5 \mathrm{~mm}$. visible between implant surface and surrounding bone around the entire femoral stem area. Peripheral to this radiolucent line there is an area of increased bone density, visible from the third to sixth month on (Fig. 48 and 49). The thickness of this radio-dense line around the entire femoral stem is approximately $1-1.5 \mathrm{~mm}$. Although the reaming effects, visible on the early explants, seemed to disappear after three months, there was in general no strong evidence of new bone formation. Slight calcar resorption was almost universally observed and general density of trabecular bone was significantly lower than that observed with hydroxyl-apatite coated implants. This difference became more evident with longer follow-up and was very marked after the two years follow-up period. In some instances there was evidence of console formation, which is bone formation distally of the femoral stem tip.

Changes in bone density are difficult to evaluate on plain radiographs. In general, a change of at least 30-50 percent in bone density is necessary to be visible on plain radiographs. Trabecular bone density of non-coated implants at longest follow-up appeared to be at best comparable to the preoperative level, but generally was somewhat lower. There was in general regular evidence of loss of cortical thickness as sign of stress-shielding osteoporosis in the proximal femur. Especially where there was bone formation distally around the implant, proximal loss of cortical thickness was observed (fig. 48).

\section{Radiographic evaluation of hydroxyl-apatite coated implants.}

During the first three weeks of follow-up, the radiographic appearance of hydroxyl-apatite coated implants is similar to that of the non-coated control implants. The bony outlines of the intra-medullary reaming procedure during surgical implantation are still visible after three weeks. With six weeks follow-up, these defects have almost universally disappeared (Fig. 50 and 51). Then, the implant-bone interface of hydroxyl-apatite coated implants has a smooth radiographic appearance. There are neither radiolucent nor radiodense lines visible around the hydroxyl-apatite coated implants. There is no visible transition zone from implant to bone. From the sixth month on, continuing until the two year follow-up period, there is an increasing difference in density and quality of trabecular bone in favor of the hydroxyl-apatite coated implants. Bone density becomes especially high around the proximal and distal femoral stem area (Fig. 52 and 53). This increase in trabecular bone density is inversely proportional to the distance from the implant. This means, bone density is highest at the coating-bone transition area. 


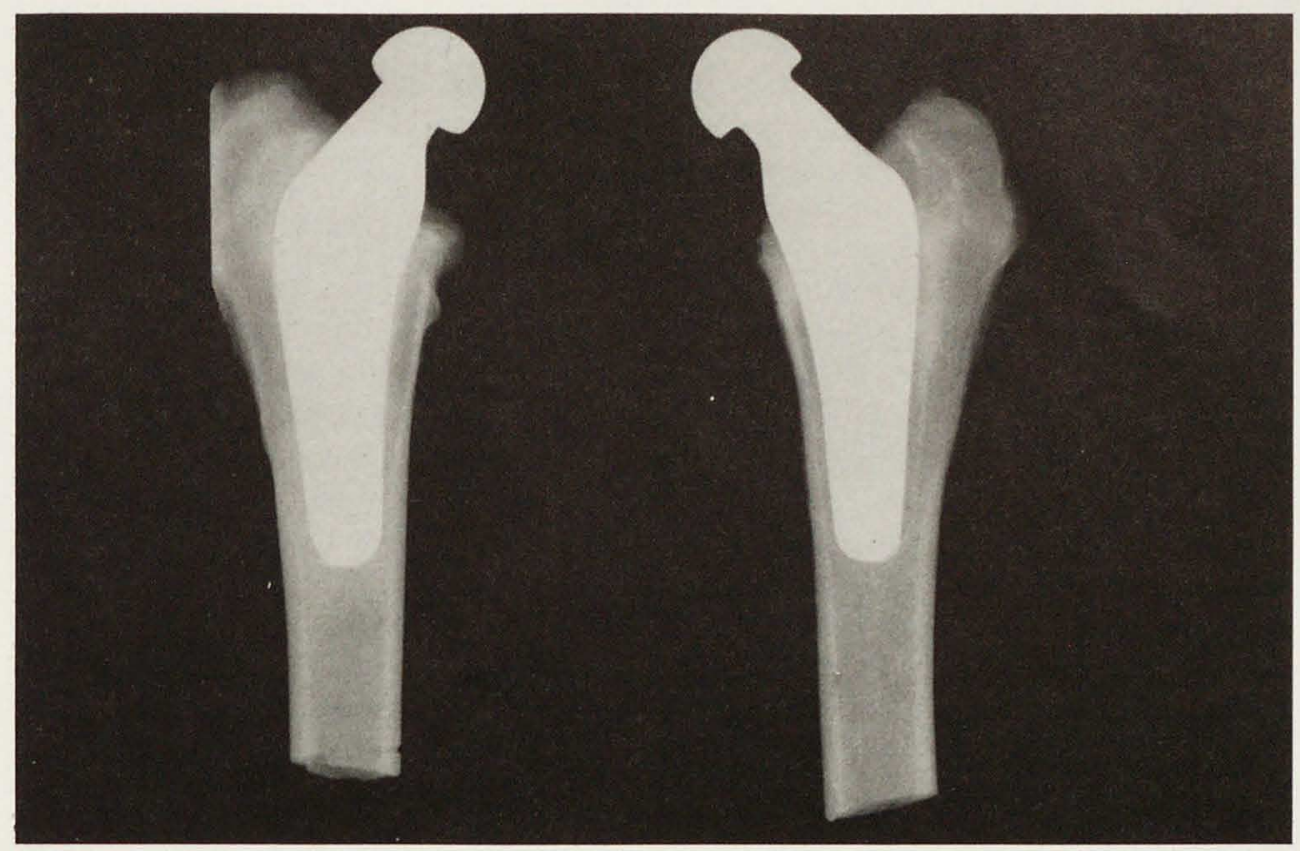

Fig. 46

Differences in radio-lucent line formation and bone density around coated (left) and non-coated (right) canine implants at one year follow-up. (AP view explant radiograph).

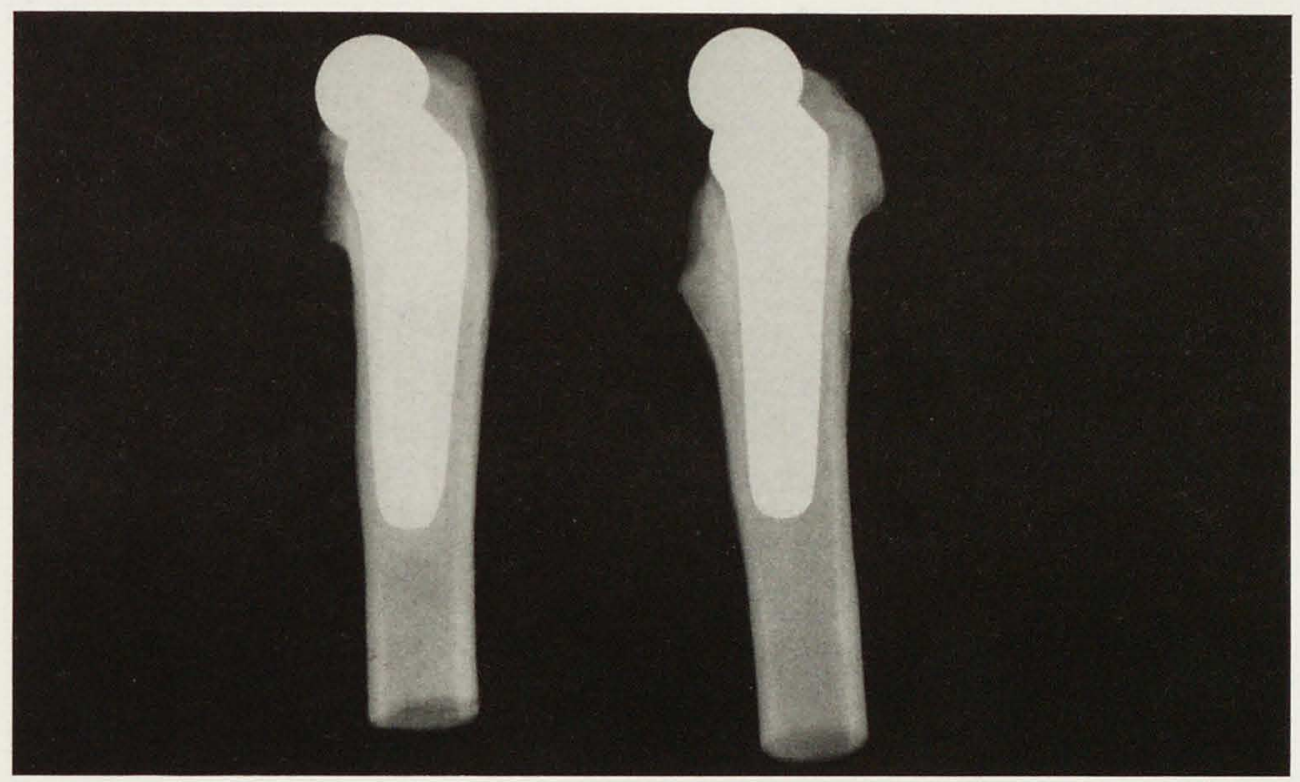

Fig. 47

Axial explant radiograph of canine hydroxyl-apatite coated (left) and non-coated (right) total hip prosthesis at one year follow-up. 


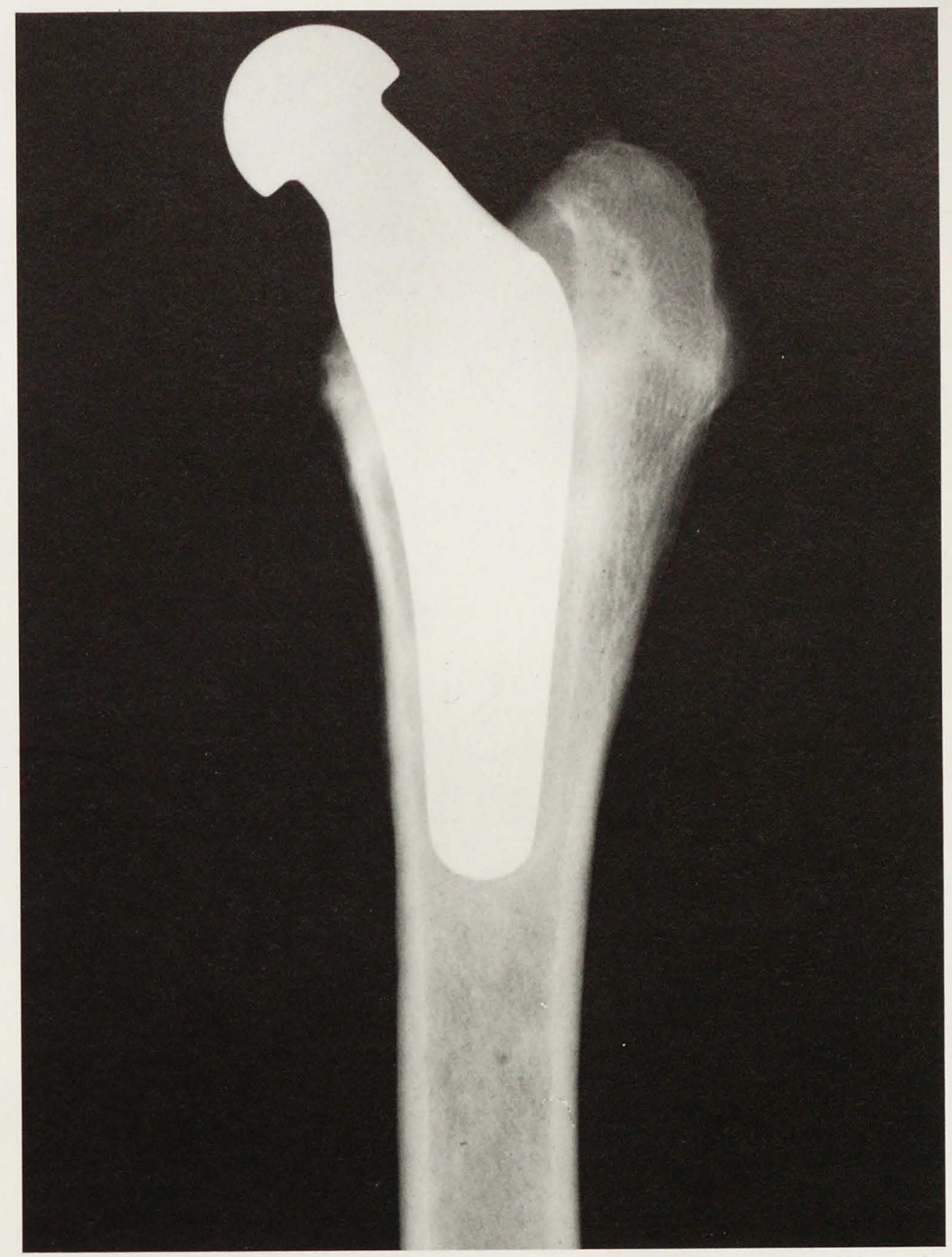

Fig. 48

Detail of radio-lucent lines and loss of trabecular structure around non-coated canine total hip prosthesis at one year follow-up. (AP explant radiograph). 


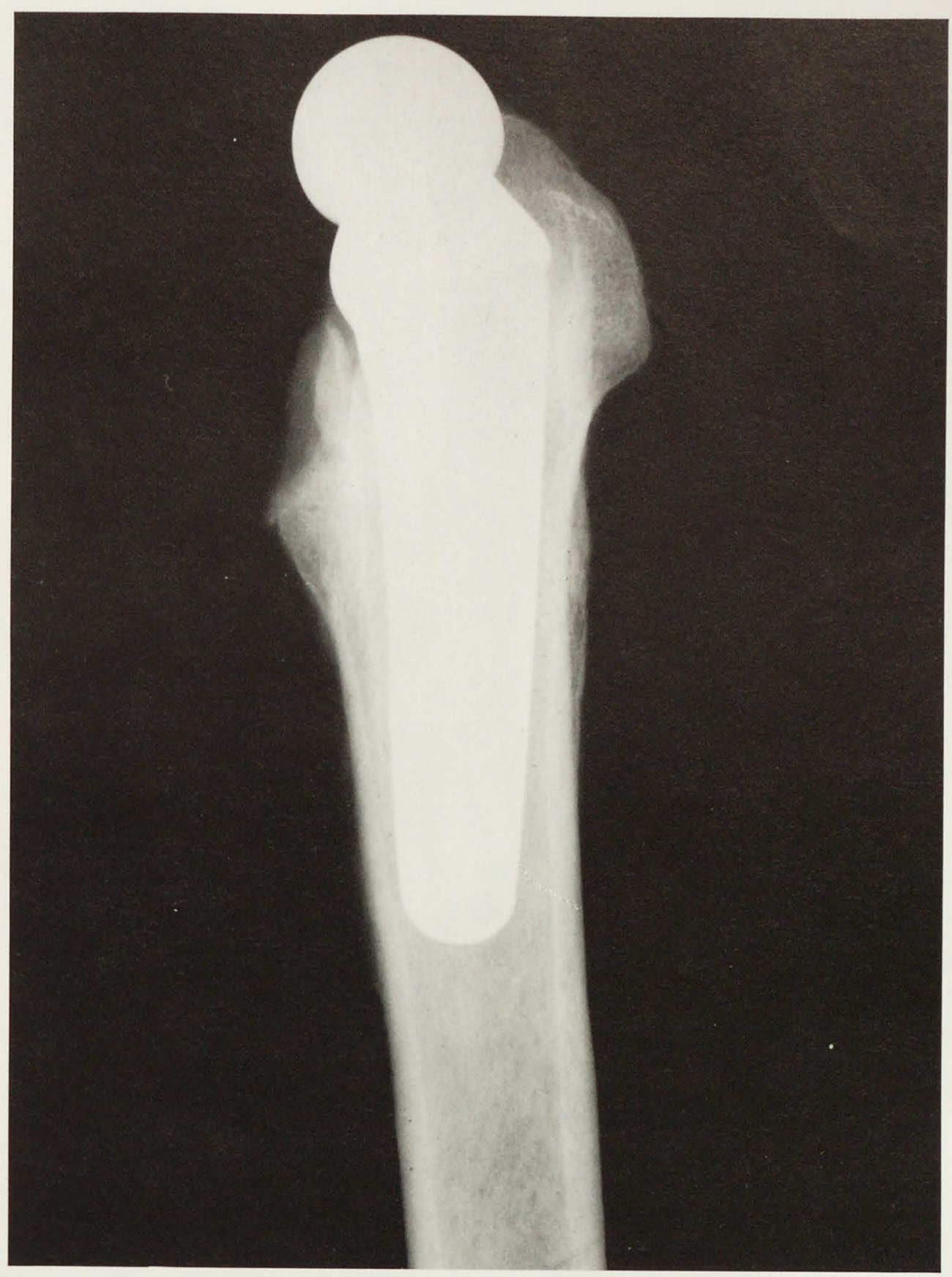

Fig. 49

Detail of radio-lucent lines and loss of trabecular structure around non-coated canine total hip prosthesis at one year follow-up. (axial explant radiograph). 


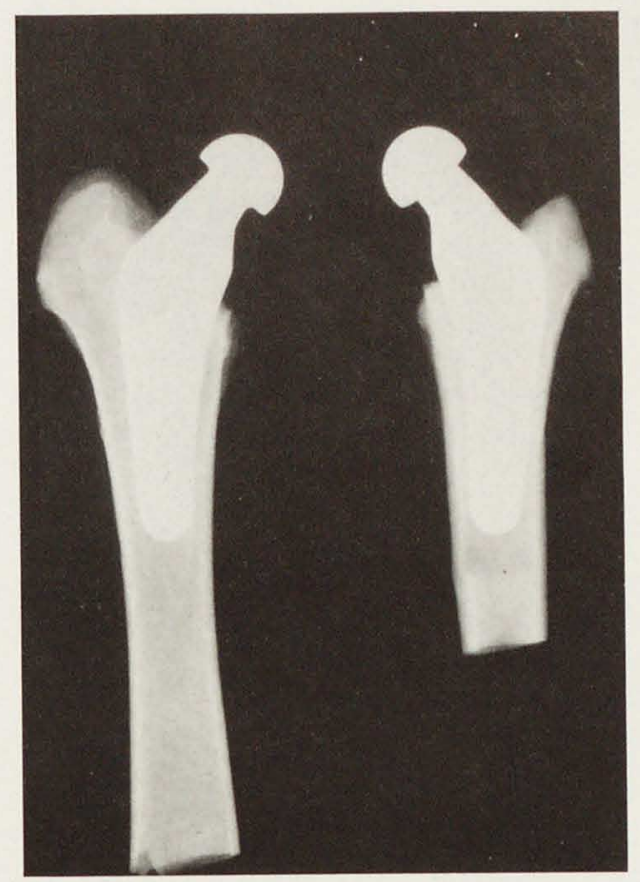

Fig. 50

Bony defects caused by reaming during surgical implantation three weeks after canine total hip replacement (right, non-coated implant) have almost disappeared at six weeks follow-up (left, hydroxylapatite coated implant, AP explant radiographs).

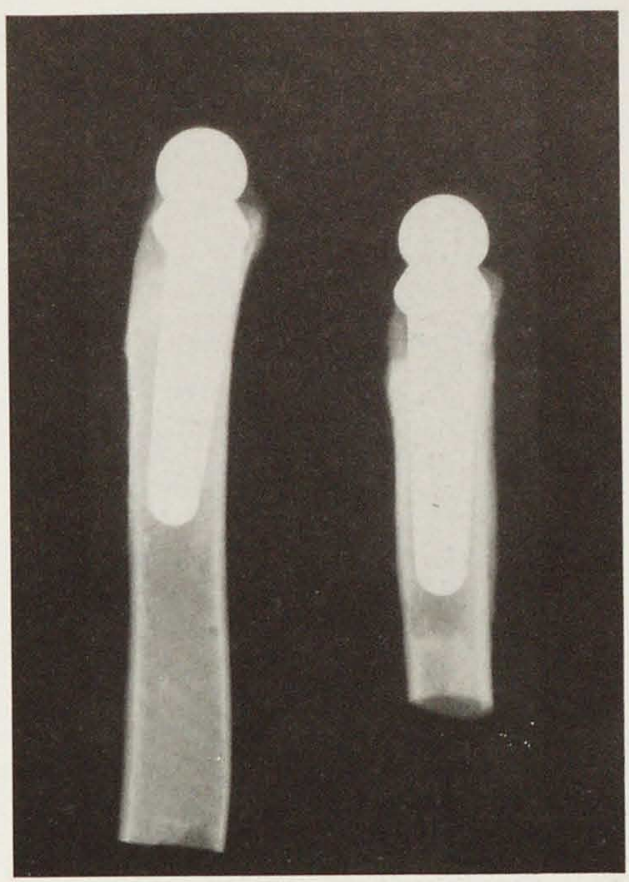

Fig. 51

Same as previous, axial explant radiographs. Hydroxyl-apatite coated implant left at six weeks, non-coated implant right at three weeks.

There are no signs of bone resorption in the direct vicinity of the implant-bone interface and trabecular bone quality is invariably good. Some implants however showed signs of loss of cortical thickness in the proximal femur, as sign of stress-shielding osteo-porosis. This phenomenon was hardly observed in the dogs with follow-up periods up to six months. Two dogs at the one year (fig. 52) and one dog at the two year interval exhibited mild loss of cortical thickness in the proximal femur, while one dog at the two year interval (fig. 54) showed more extensive loss of femoral cortex in the medial proximal femoral area. In the first mentioned three cases with mild stress-shielding effects, there was good trabecular bone densification in the proximal femur, while in the last case with more extreme stress-shielding osteoporosis, there was more extensive trabecular bone densification around the distal femoral stem and hardly any trabecular bone densification in the proximal area of the femur. The femoral cortex has almost disappeared in this area, while the remaining cortex shows spongialization of same around the implant. There were no other radiographic signs of adverse effects of the hydroxyl-apatite coating on the bone neither abnormal calcifications. 


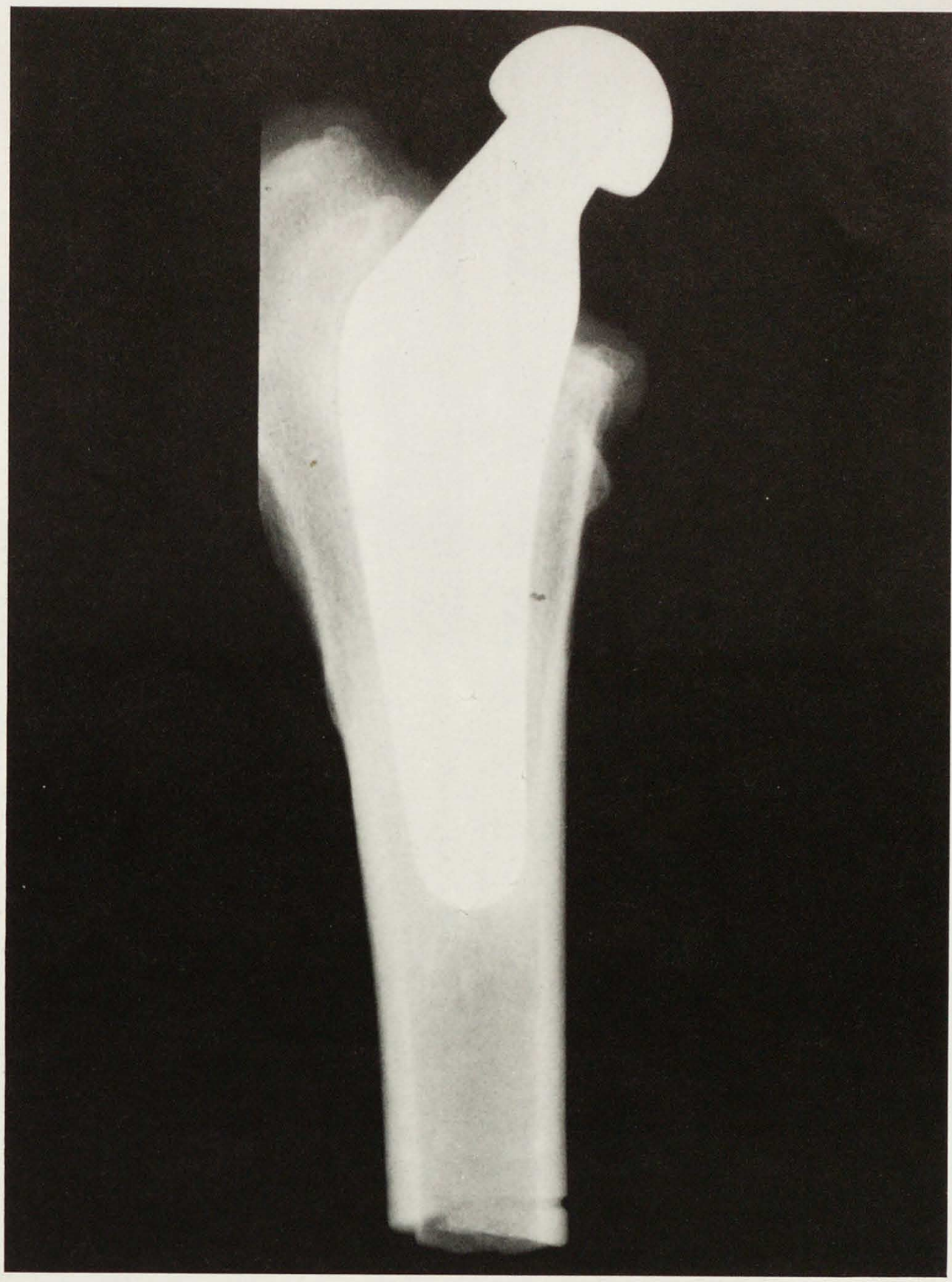

Fig. 52

Bone densification around hydroxyl-apatite coated implants is especially evident in proximal and distal stem area at one year follow-up. Moderate loss of cortical wall thickness in proximal femur (AP explant radiograph). 


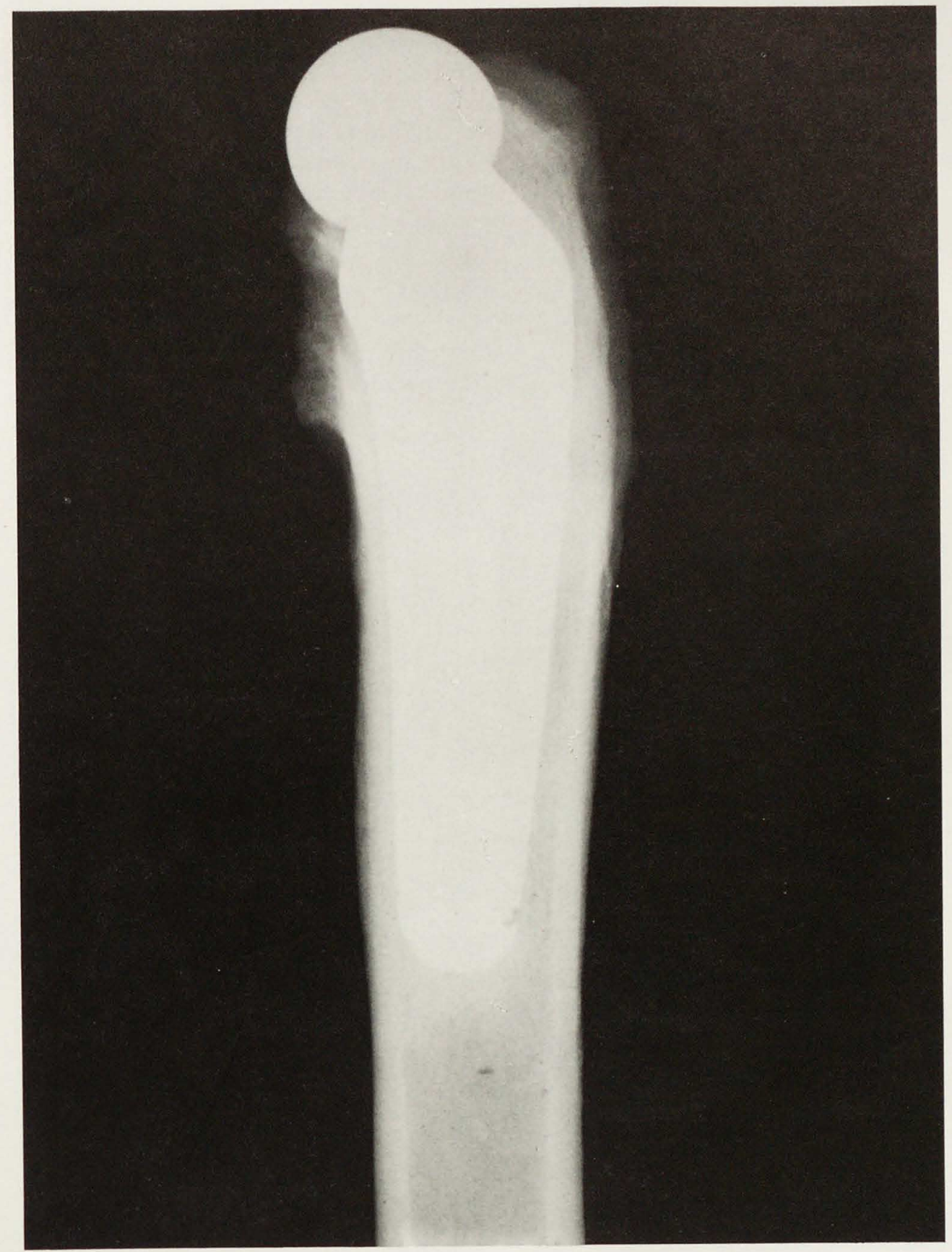

Fig. 53

Bone densification around hydroxyl-apatite coated implants is especially evident in proximal and distal stem area at one year follow-up. (axial explant radiograph). 


\section{Scintigraphic evaluation of prostheses}

\section{Scintigraphic evaluation of non-coated implants}

All 99m-technetium bone scans on non-coated implants with six month to two year followup show a characteristic three point hot spot with radio-nuclide accumulation (Fig. 54, 55, 56 and 57). These areas with increased $99 \mathrm{~m}$-technetium uptake include the medial and lateral aspects of the proximal femur as well as the area around the femoral stem tip. The areas in between of these hot spots do not exhibit as much uptake as compared to the corresponding areas in their hydroxyl-apatite coated counterparts.

\section{Scintigraphic evaluation of hydroxyl-apatite coated implants}

The scintigraphic pattern of hydroxyl-apatite coated implants after the sixth month of follow-up is characterized by a moderate uptake of $99 \mathrm{~m}$-technetium with an even distribution of activity over the entire proximal femur. There are no hot spots or other focal accumulations of the radio-nuclide, neither proximally nor distally in the femur. In

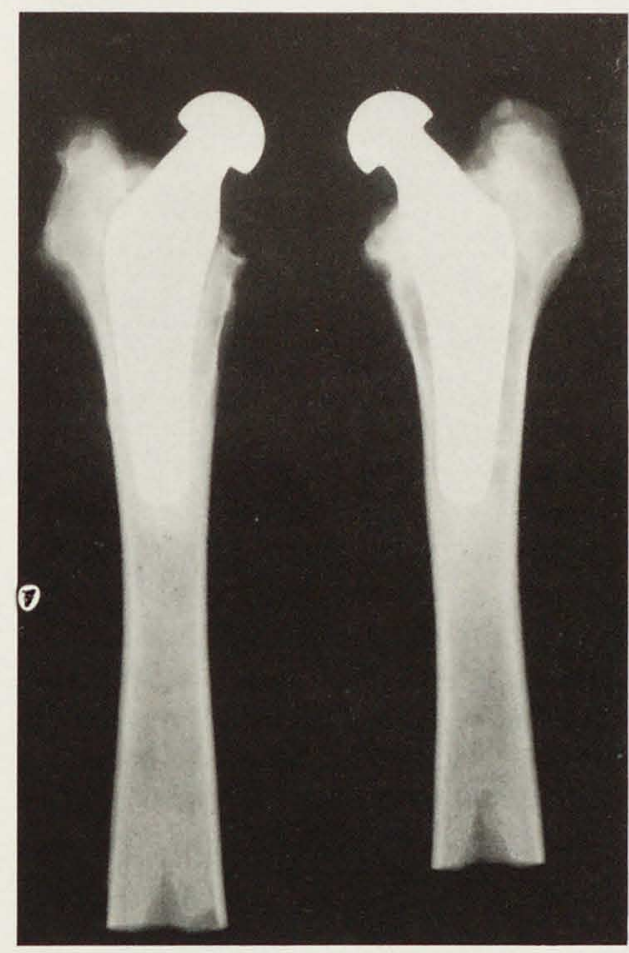

Fig. 54

AP explant radiograph of hydroxyl-apatite coated (left) and non-coated (right) implant, showing differences in bone density and trabecular structure, especially around the distal stem area at two years follow-up. Observe stress-shielding effects with thinning of proximal femoral cortex on left coated explant.

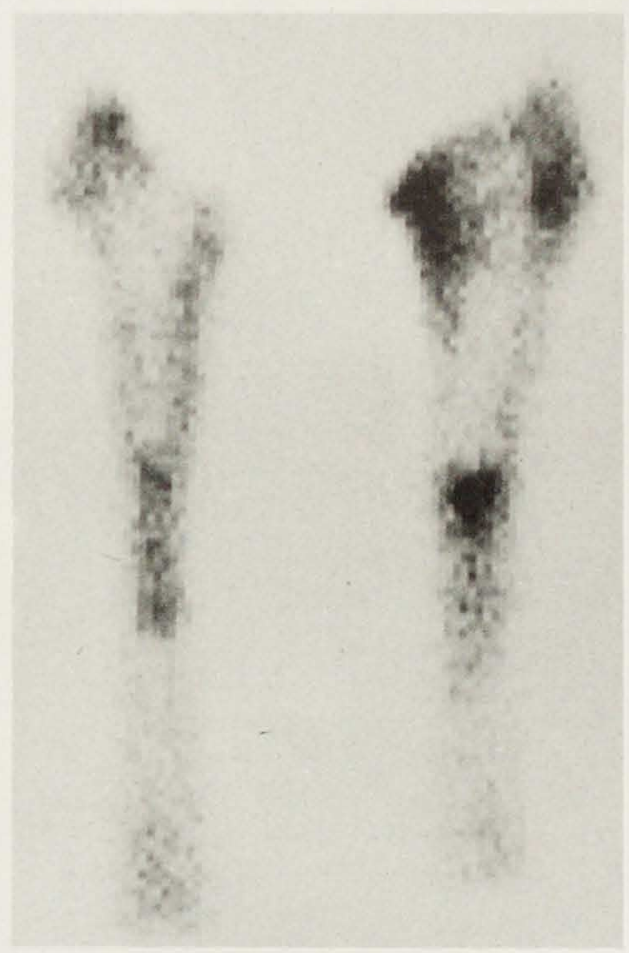

Fig. 55

AP view of $99 \mathrm{~m}$-bone scintigram of hydroxyl-apatite coated prosthesis (left) and non-coated prosthesis (right) at two years follow-up. Same implants as previous radiograph. Even distribution of technetium activity over bone mass around coated implant. Three point hot spot localization over noncoated implant. 


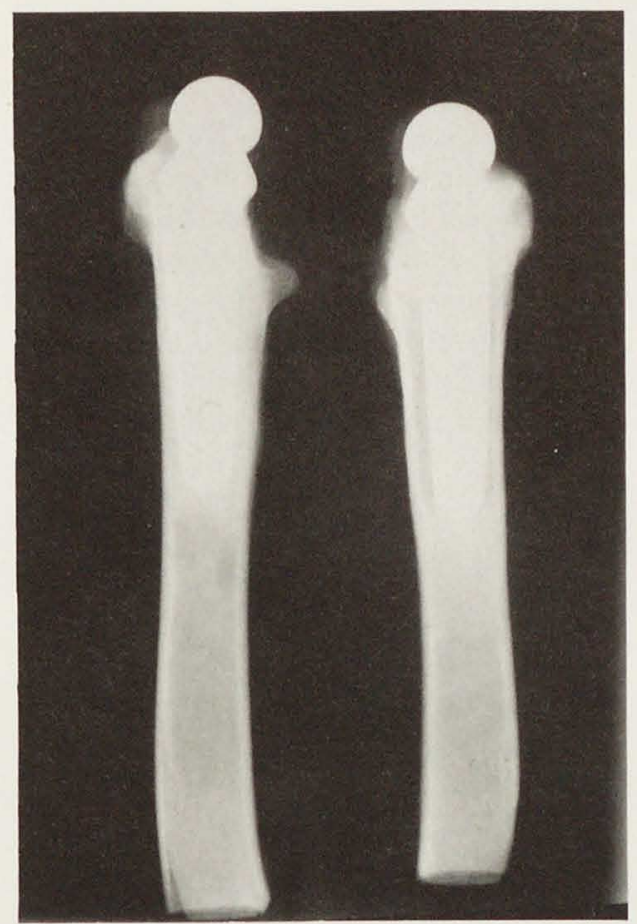

Fig. 56

Axial explant radiograph of hydroxyl-apatite coated (left) and non-coated (right) implant, showing differences in bone density and trabecular structure, especially around the distal stem area at two years follow-up.

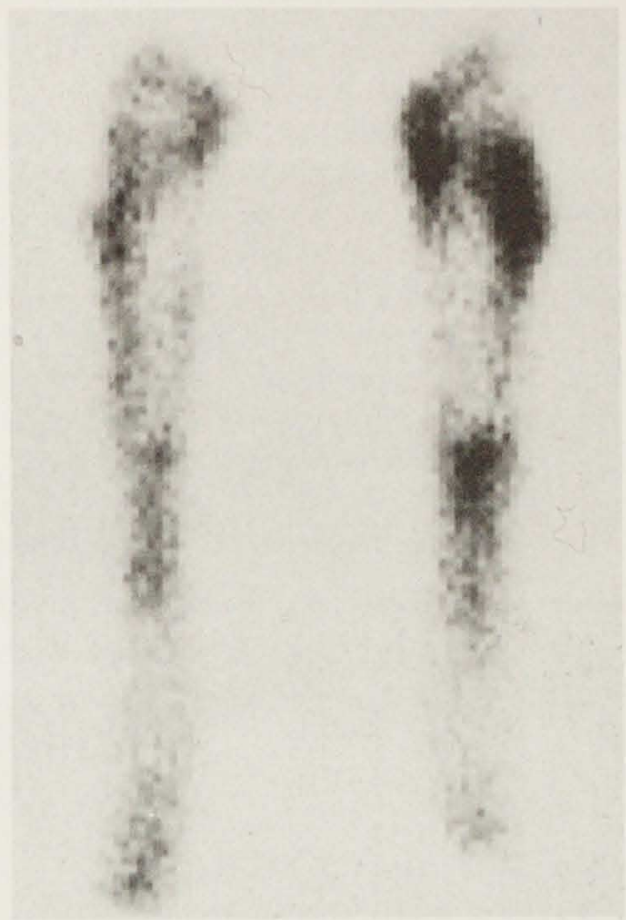

Fig. 57

Axial view of $99 \mathrm{~m}$-bone scintigram of hydroxylapatite coated prosthesis (left) and non-coated prosthesis (right) at two years follow-up. Same implants as previous radiograph. Even distribution of technetium activity over bone mass around coated implant. Three point hot spot localization over noncoated implant.

general 99m-technetium uptake was somewhat more elevated in areas of bone that exhibited endosteal condensation of bone. This means, $99 \mathrm{~m}$-technetium uptake has a somewhat more or less linear relationship to the actually present calcified bone mass. The figures represent radiological and scintigraphic comparisons between an hydroxyl-apatite coated implant after two years follow-up. There is endosteal condensation of bone in the area of the distal femoral stem around the fully coated stem. $99 \mathrm{~m}$-Technetium uptake is somewhat higher in this area, but nowhere is there any hot spot formation. Also, the axial views show the $99 \mathrm{~m}$-technetium uptake is where the bone mass is. Radio-nuclide uptake over the proximal femur has a very even distribution of uptake, which is generally somewhat higher as compared to the non-coated control implant in the same area outside of its hot spots. 


\section{Mechanical evaluation of prostheses.}

With implants of complex design, like hip prostheses, it is impossible to quantify exactly the mechanical fixation to bone. The plug implant study served that purpose.

Nevertheless, there was a striking difference in extraction force for removal of implants from the femur. Uncoated implants were easily extracted from the femur at any postoperative time. With hydroxyl-apatite coated implants, this became increasingly difficult after three weeks of implantation. Hammer and chisel were required to remove the implant and a fracture occurred with an attempt to remove a coated implant from the femur after six weeks (Fig. 58). Additionally, it shows the good bone quality around the coated hip stem, even after this relative short follow-up period. No other forceful attempts at removal of hydroxyl-apatite coated implants were undertaken, because it renders histological evaluation impossible.

\section{Micro-radiography of histological sections.}

Micro-radiographic examination of histological sections shows a difference in interface characteristics between hydroxyl-apatite coated and uncoated implants.

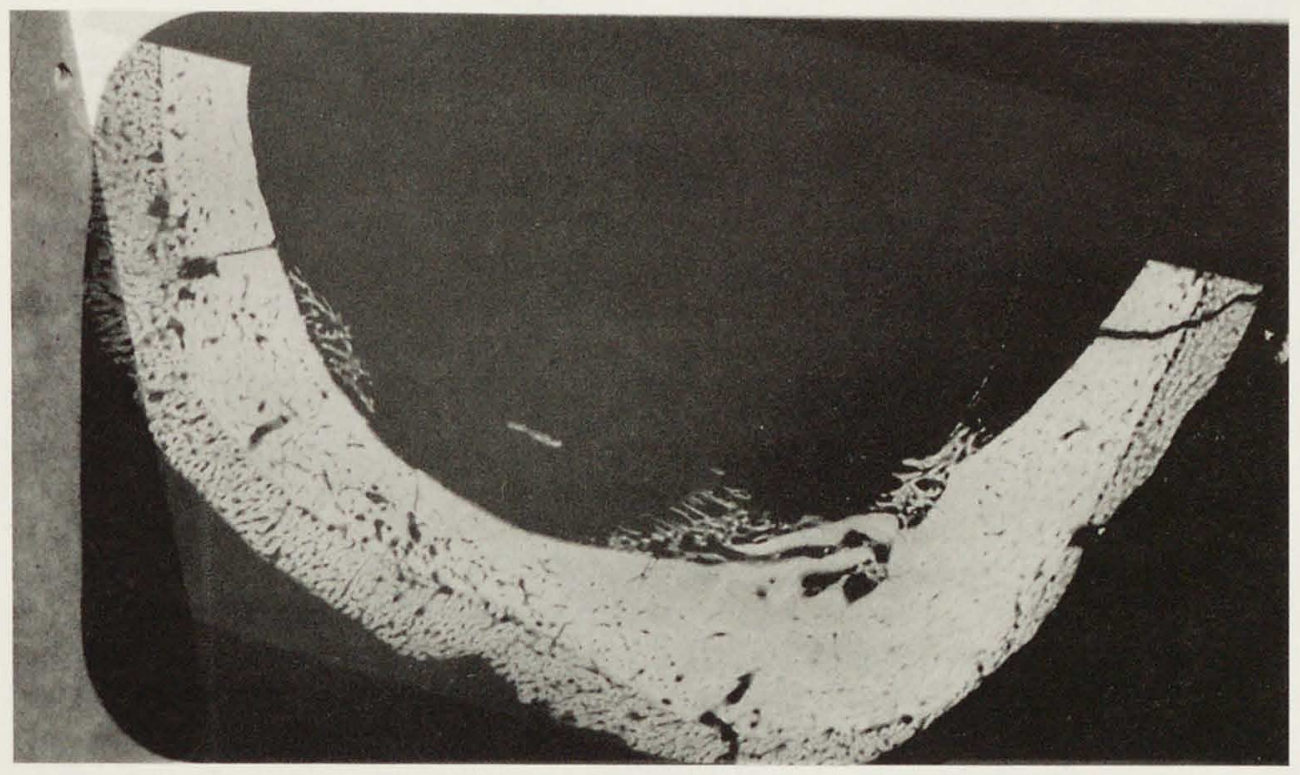

Fig. 58

Fissures, fractures and pull-out of bone after forceful extraction of hydroxyl-apatite coated prosthesis from femur at six weeks. (Micro-radiograph, x1).

\section{Micro-radiography of non-coated prostheses.}

Non-coated prostheses have an irregular ending of bone some distance away from the implant. With time there is an increasing loose area between implant and bone. After two years follow-up, the distance between implant and bone can be as much as $1-1.5 \mathrm{~mm}$. The orientation of the bone trabeculae is in general parallel to the implant surface. The bone trabeculae are much smaller as compared to the hydroxyl-apatite coated implants. Also 
their radio-density declines in the direction of the implant. There is no separate radiodense area around non-coated implants.

\section{Micro-radiography of hydroxyl-apatite coated prostheses}

With hydroxyl-apatite coated prostheses bone is present up to the implant surface. After three weeks follow-up there is already a thin lining of bone visible on the implant surface (Fig. 59 and 60). This is especially clear when the implant metal is removed from the microscopic sections. The bony architecture closely follows the configuration of the prosthesis. The ending towards the implant surface is therefore very flat. At the six week interval no slits and spaces are visible in the main weight-bearing part of medial calcar area. Also the ventral, dorsal and lateral parts of the prosthesis have close bone contact, although the bone is thinner there. The structure of the bone trabeculae is fully interconnective. The individual bone trabeculae are thicker as compared to the non-coated implants. There is an increase in radio-density of the bone towards the implant surface by an increase in calcium-content of the bone. With time the radio-density of the bone will further increase and after two years follow-up, the trabecular bone is very dense, almost cortical in character, around the major part of the entire stem area. As already mentioned with explant radiography, one hydroxyl-apatite coated implant showed more extreme loss of cortical thickness in the medial proximal femur, as sign of stress-shielding osteoporosis.

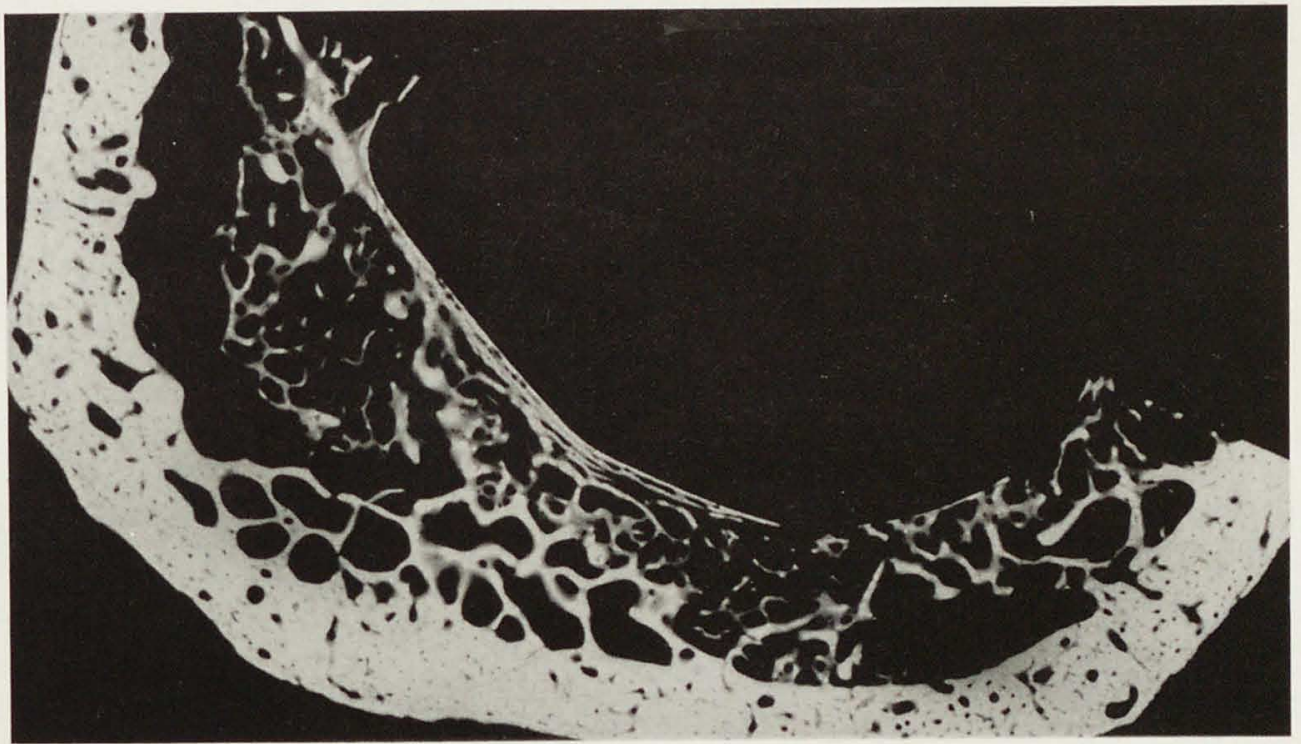

Fig. 59

Thin layer of bone and connecting trabeculae on hydroxyl-apatite coated implant surface at three weeks followup. (Micro-radiograph of proximal stem area cross-section, x1). 


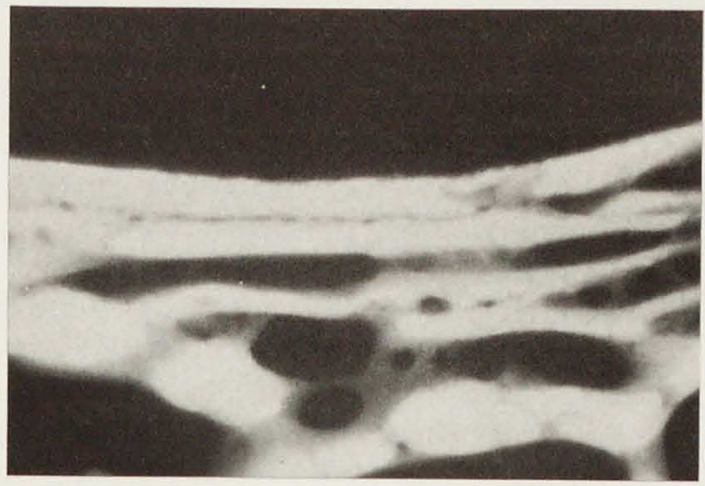

Fig. 60

Same as previous, magnification $\times 50$.

\section{Histological evaluation of prostheses.}

\section{General comments}

With implants of complex geometry there are always areas with bone gaps and spaces. Especially around the conical distal end of the stem slits and spaces are likely to occur, despite good instrumentation and surgical technique. This was reflected in the histological results. Early transverse sections through the proximal femur of both coated and noncoated implants, reveal that the initial mechanical stabilization of the prosthesis was achieved by three areas of point contact between implant and bone (compare fig. 45). This occurred generally at the antero-medial, postero-medial and postero-lateral corners of the prosthesis. Also, the calcar area shows rather close initial bone contact. When calculated, the initial surface contact area around the implant is at best 20 percent, but most of the time even less than that. Although both types of implants have the same initial pressfit fixation, there develops a striking difference in histology between coated and non-coated prostheses.

\section{Cemented acetabular cups}

Cemented cups were used as the acetabular component in canine total hip replacement. Although not specifically the purpose of the study, some of the cemented components showed radiological and histological signs of implant loosening. Three acetabular components (two of an uncoated and one of a hydroxyl-apatite coated implant) were clinically loose at the time of retrieval. Virtually all acetabular components showed histologically a fibrous-tissue interface with signs of bone resorption at the cement-bone interface. The thickness of the fibrous-tissue membrane was variable, ranging between a few cell-layers and 0.5 millimeter. There were regularly macrophages and other giant-cells seen in the interface area between cement and bone. These appeared to play an active roll in the process of bone resorption around the implant (Fig. 61). These findings are in agreement with those of other authors (Paul ea. 1987), who invariably found a fibrous tissue interface around cemented acetabular cups in experimental arthroplasties, even using modern cementing techniques. 


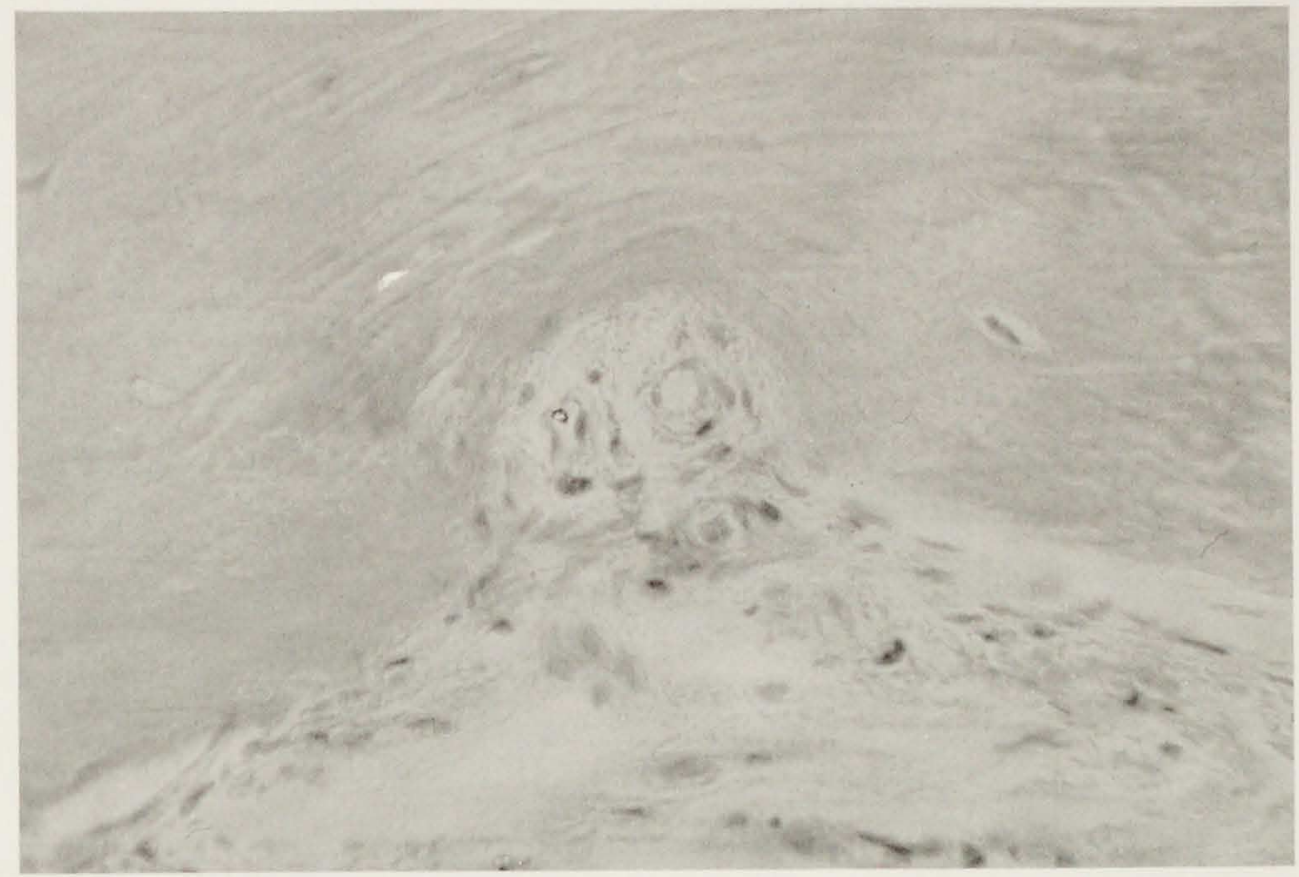

Fig. 61

Bone-implant interface of cemented canine acetabular cup. Fibrous tissue layer with multi-nulear giant-cells. (HE, six months, x200).

\section{Histology of non-coated titanium prostheses}

\section{general evolution of bone processes around uncoated implants}

The general evolution of the interface between uncoated titanium implants and bone is one towards fibrous-tissue encapsulation of the implant. There is predominant bone resorption at the implant-bone interface of non-coated implants and although this will be counter-acted by the remaining bone with increased bone formation, the balance between bone formation and bone resorption is still delicate after two years.

\section{implant-bone interface of non-coated implants}

Non-coated prostheses are surrounded by a fibrous tissue membrane with a thickness between 100 and 200 microns. This fibrous tissue is visible from the third week on and becomes more prominent with time. In addition there is an empty space between fibrous tissue and implant, indicating motion between the two (Fig. 62). This empty space has a thickness of up to $1.5 \mathrm{~mm}$. After two years the entire femoral stem area is encapsulated in a fibrous-tissue membrane. The end-thickness of this membrane is rather variable and between 100-500 microns in thickness. The bone trabeculae adjacent to the fibrous tissue membrane tend to have more parallel orientation towards the implant surface (Fig. 63). In the microscopical sections of non-coated titanium implant-bone interfaces there were no macrophages or related cells, apart from osteoclasts, that seemed to play a role in the process of bone resorption. 


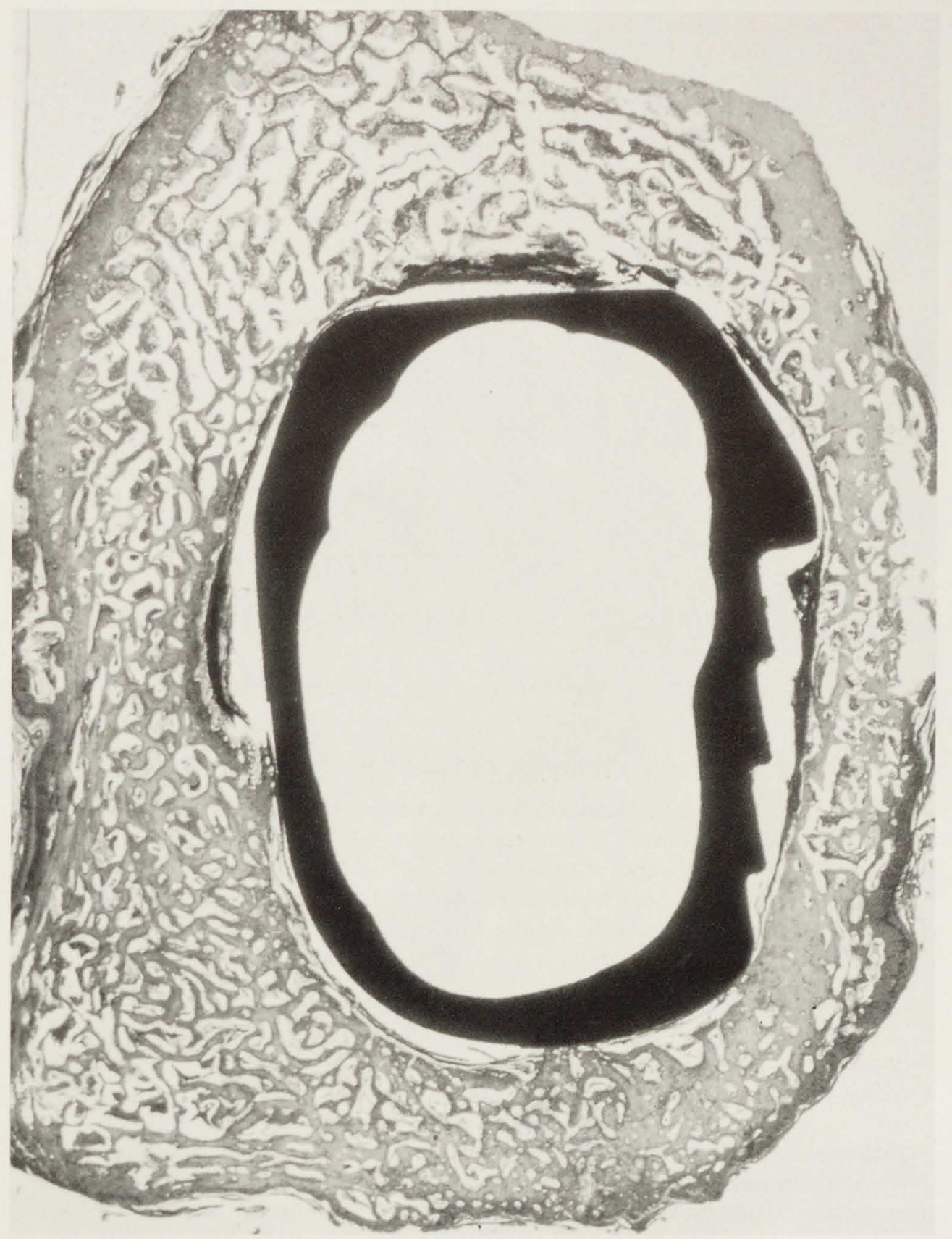

Fig. 62

Loose area and fibrous tissue around non-coated canine femoral component in proximal stem area. Observe stress-shielding effects: loss of cortical wall and spongialization of cortical bone with transition to almost trabecular bone at medial side of proximal femur. (Masson trichrome, one year, $\mathrm{x} 1$ ). 


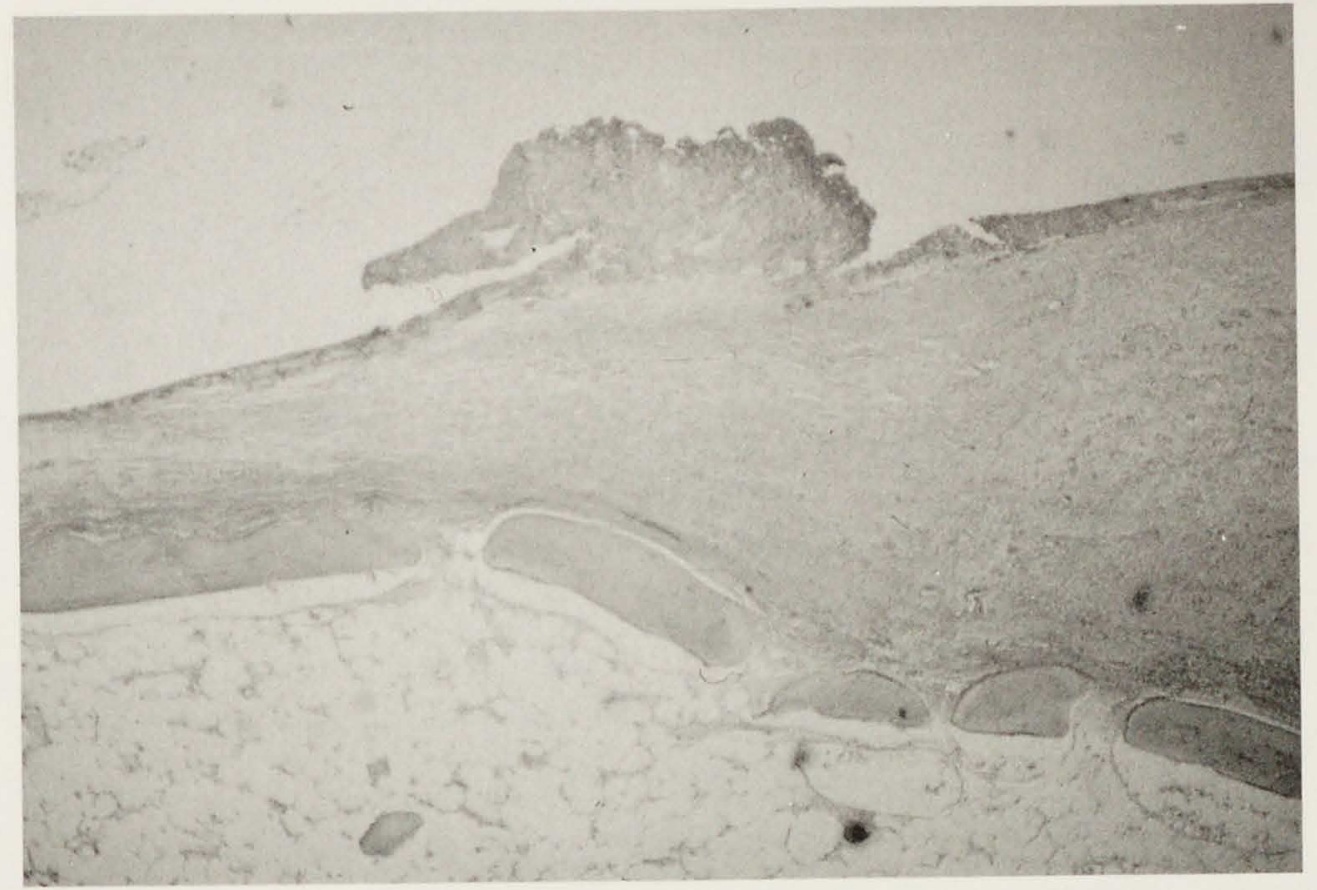

Fig. 63

Parallel orientation of bone trabeculae to implant surface and fibrous-tissue interface around proximal femoral stem of non-coated implant (Alcian-blue, six months, $\mathrm{x} 5$ ).

\section{new bone formation around non-coated implants}

From the histological sections there is few evidence of new bone formation. In the proximal and distal femoral stem area, there are initially bone defects caused by the intramedullary reaming during surgical implantation. These defects may become smaller with time at some distance from the implant. Close to the implant surface fibrous tissue will take the place of the bone. The only other evidence of new bone formation is the one in reaction to increased bone resorption. As a reaction on the prevailing bone-resorptive processes around non-coated implants, there is a reaction of the bone towards bone proliferation. This occurs directly below the resorption front at the bone/fibrous-tissue transition area and is visible from the third month on (Fig. 64 and 65). Although the histology shows a layer with increased bone proliferation, the net result is nil, because bone resorption keeps pace with this new bone proliferation.

\section{bone resorption around non-coated implants}

The first six to twelve weeks there is predominant bone resorption. The bone trabeculae have lost their osteoid zone at the side, facing the prosthesis. Remnants of bone trabeculae are visible throughout this area near the implant surface (Fig. 66). Fibrous tissue replaces the resorbed bone. Polarization microscopy still discloses remnants of calcified tissue within this area of fibrous tissue in this period (Fig. 67 and 68). Later on this phenomenon is absent. From the third month on, the implant-bone interface stabilizes to some extent. 


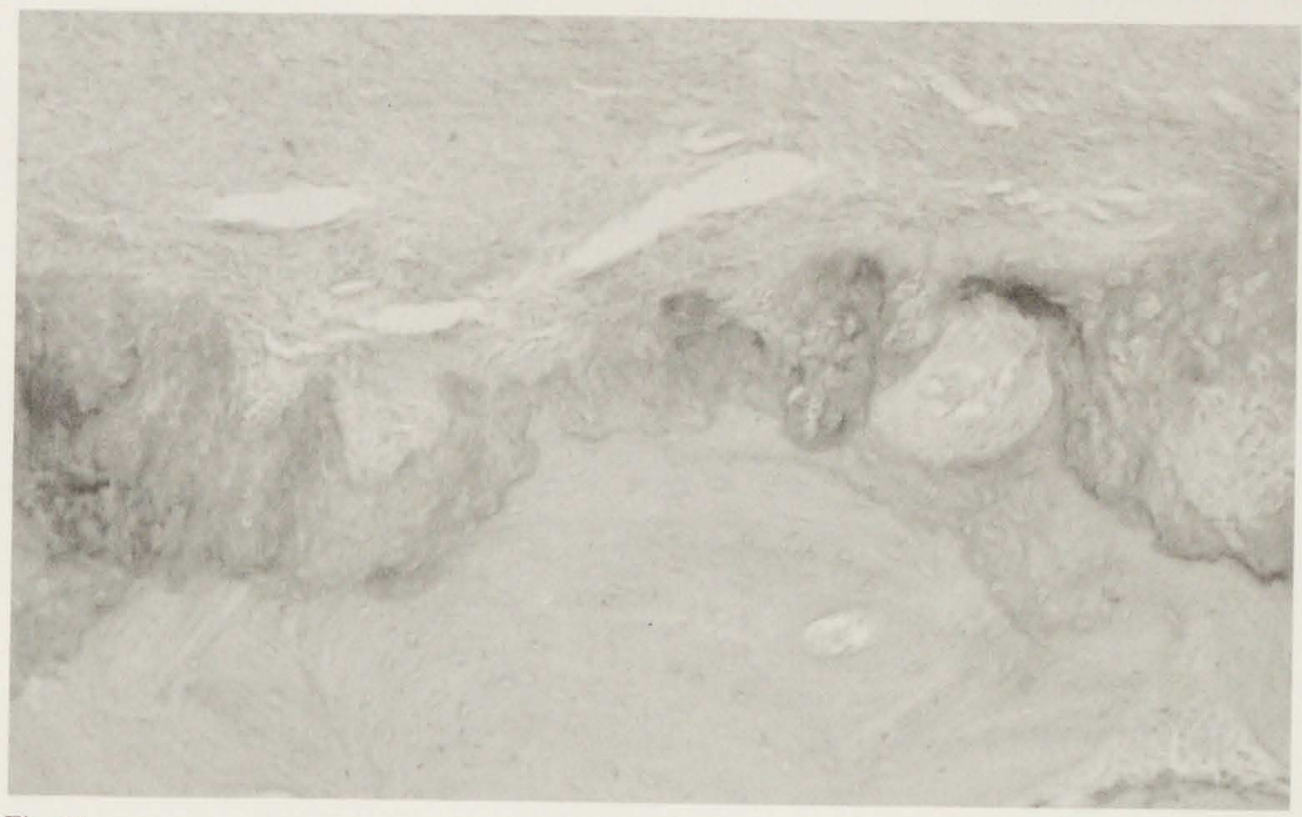

Fig. 64

Reactive proliferation of bone with osteoid layer below fibrous-tissue layer of non-coated canine total hip prosthesis. (Alcian-blue, six months, $\mathrm{x} 50$ ).

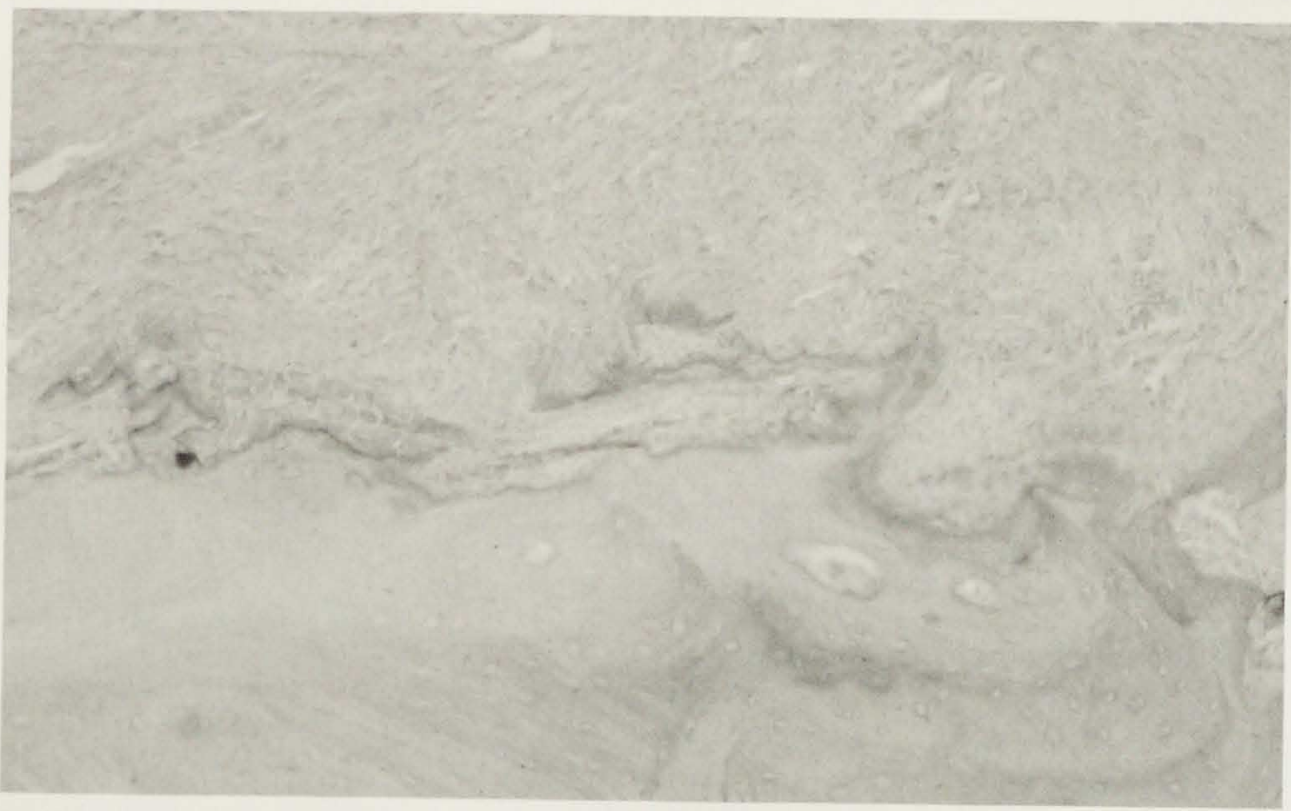

Fig. 65

Reactive proliferation of bone with thin osteoid layer below fibrous-tissue layer of non-coated canine total hip prosthesis. (Alcian-blue, two years $\times 50$ ). 
Then, there is an irregular osteoid zone, indicative of new bone formation, at the transition area between fibrous tissue and underlying bone. The net result after one or two years appears as if bone formation and bone resorption balance each other. The fibrous tissue layer remains about 100 to 500 microns thick, also in the proximal calcar area. Several implants, especially with the longer follow-up periods of one and two years exhibited signs of loss of cortical thickness or spongialization of cortical bone, as sign of stress-shielding osteo-porosis (fig. 54). These phenomena vary between slight loss of cortical thickness and slight cyst formation in the cortical bone to almost complete loss of cortical thickness with transition to more loose trabecular bone. The initially small cysts in the cortical bone enlarge and, with continued remodelling, there is a gradual transition to almost trabecular appearance of the bone (fig. 62). Localization of these changes was almost invariably, the medial proximal femoral cortex around the implant.

\section{general density and structure of bone around non-coated implants}

The bone trabeculae are rather thin as compared to those around hydroxyl-apatite coated implants. This is apparent from the third month on and becomes more evident after this time interval. In general, the remaining bone trabeculae tend to have more parallel orientation towards the implant surface (Fig. 63). There is no histologic parallel to the observed radiological phenomenon of radio-dense line formation at some distance around non-coated prostheses.

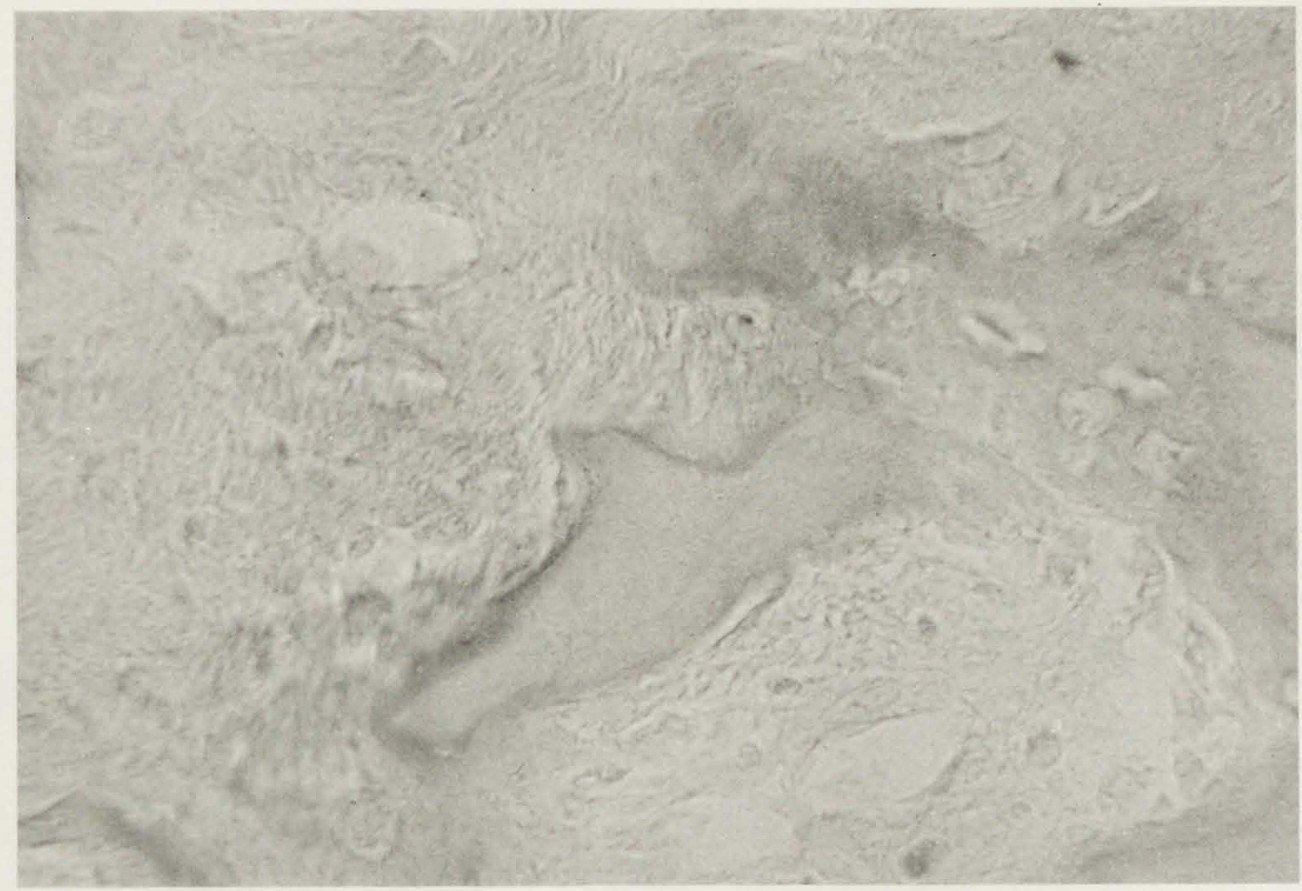

Fig. 66

Remnants of resorbing bone trabeculae within fibrous-tissue membrane of non-coated canine total hip implant. (Alcian-blue, six weeks, $\mathrm{x} 100$ ). 


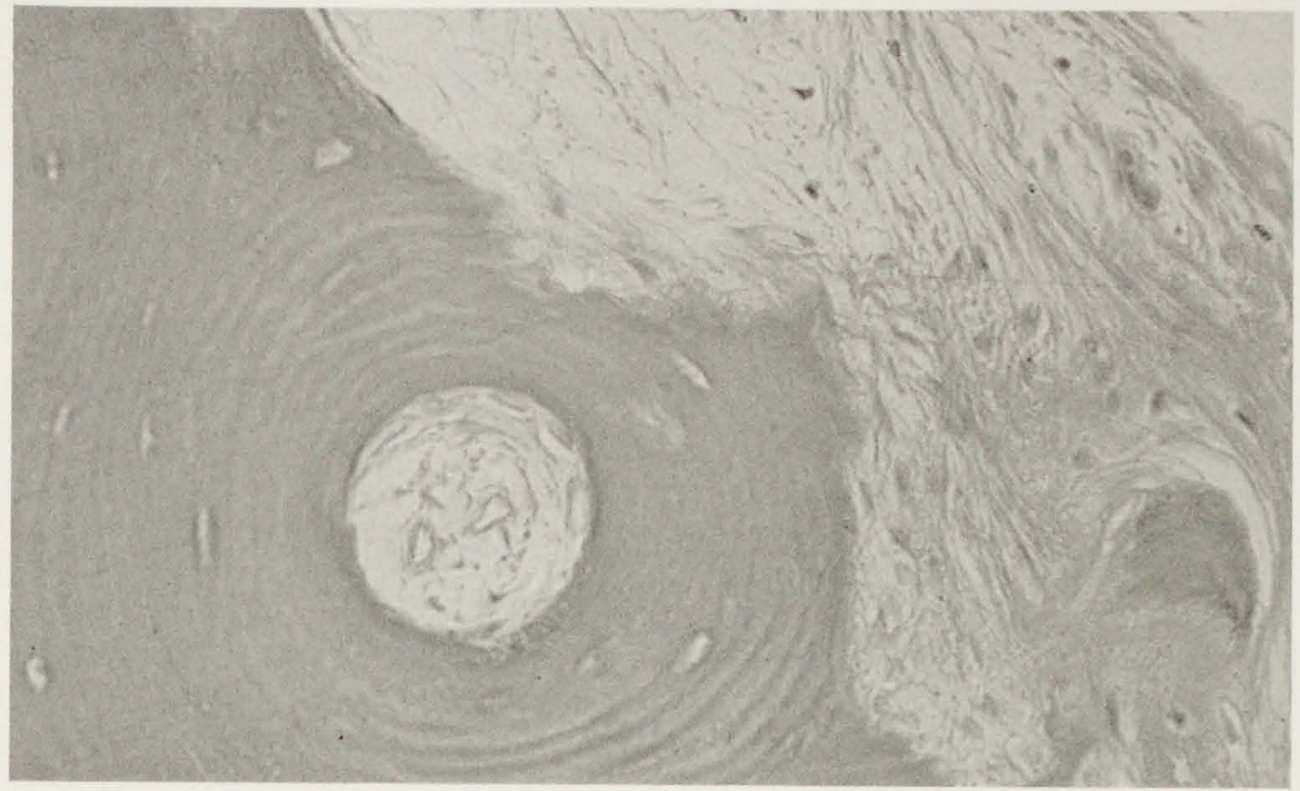

Fig. 67

Haversian system near non-coated femoral stem in process of resorption, apparent fibrous tissue in upper right corner (HE, six weeks, x200).

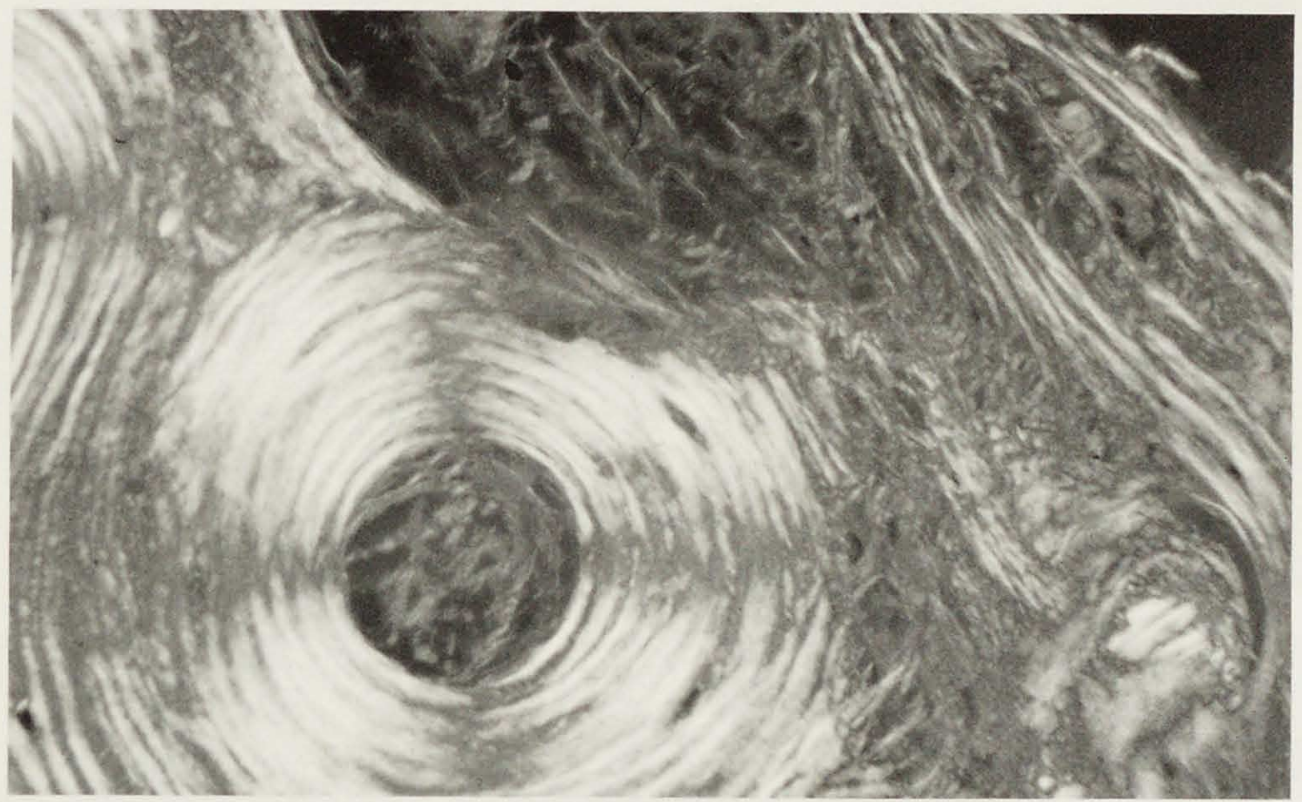

Fig. 68

Same as previous, haversian system near non-coated femoral stem in process of resorption, showing remnants of calcified material in fibrous tissue near implant-bone interface in right upper corner. (HE with polarization, six weeks, x200). 
potential of non-coated implants to fill bone defects

The initially present bony defects, slits and spaces around the implant are still there after follow-up of up to two years. Although there is some remodelling and filling of bony defects at distance of the non-coated implant, the bony defects in close proximity to the implant become integrated in the general process of fibrous encapsulation of non-coated implants.

\section{differences in response of non-coated titanium implants with cortical or trabecular bone}

Both cortical and trabecular bone tend to stay away of the non-coated implant. After two years follow-up cortical bone of the femoral shaft is often demineralized to some degree, although never excessive. The trabecular bone is thin and subject to resorption.

\section{response of bone-marrow cells close to non-coated implants}

Other parameters for implant acceptance include the histology of the bone-marrow around the implant, where still present. In the proximal femur the bone-marrow has largely been reamed away. The initial cell response after three to six weeks is inflammatory with predominant presence of polymorpho-nuclear leukocytes. After three to six months and later, the regenerate cells around the non-coated implant have a more normal appearance. Mainly lymphocytes and plasma-cells are present with scarcely a polymorphonuclear-leucocyte visible. The findings are comparable to those of the plug implant study.

response of prosthetic neo-capsule around non-coated implant

The prosthetic neo-capsule has the normal appearance of fibrous tissue. Initially, the superficial zone contains multiple cell-layers. After six months this lining layer has a thickness of only a few cells. The findings are rather similar to those of hydroxyl-apatite coated implants. There are no pathological inclusions, nor abnormal calcifications in the prosthetic neo-capsule.

\section{Histology of hydroxyl-apatite coated prostheses}

general evolution of implant-bone interface with hydroxyl-apatite coated implants General evolution of hydroxyl-apatite coated implant-bone interface is towards complete bony coverage of implants. Within three weeks hydroxyl-apatite coated implants develop a close contact with the surrounding bone. Many bony defects, slits and spaces have closed by the three week interval, especially in the proximal stem area. At six weeks postoperatively the coated implants have a ninety-five to near one hundred percent covering with bone (Fig. 69). Trabecular bone density around the implants increases with time until an almost cortical character of same.

character of implant-bone interface with hydroxyl-apatite coated prostheses The characteristic interface picture of a hydroxyl-apatite coated implant consists of a bone-plate against the implant of varying thickness (Fig. 70). This depends on time and loading conditions. After three weeks there is clear evidence of mineralized bone apposition against the hydroxyl-apatite coating. This bone plate is initially very thin, some 100-300 microns, but becomes rapidly thicker as time progresses. The ultimate interface consists for a near hundred percent of mature dense bone. High magnification views 
(1000x) confirm the close bonding between hydroxyl-apatite coating and bone without any interposition of fibrous tissue. The transition area of bone to coating shows apposition of osteoid material on the hydroxyl-apatite coating in the early follow-up period, (Fig. 71) followed later by maturation to bone and increased mineralization at the longest followups of one and two years (Fig. 72). These areas correspond to those of increased calcium content as seen in micro-radiographs. Close approximation of hydroxyl-apatite coatings is not only seen with mature osteocytes, but also with young osteoblasts (Fig. 73). Many haversian systems show direct endings on and bonding with the hydroxyl-apatite coating (Fig. 74).

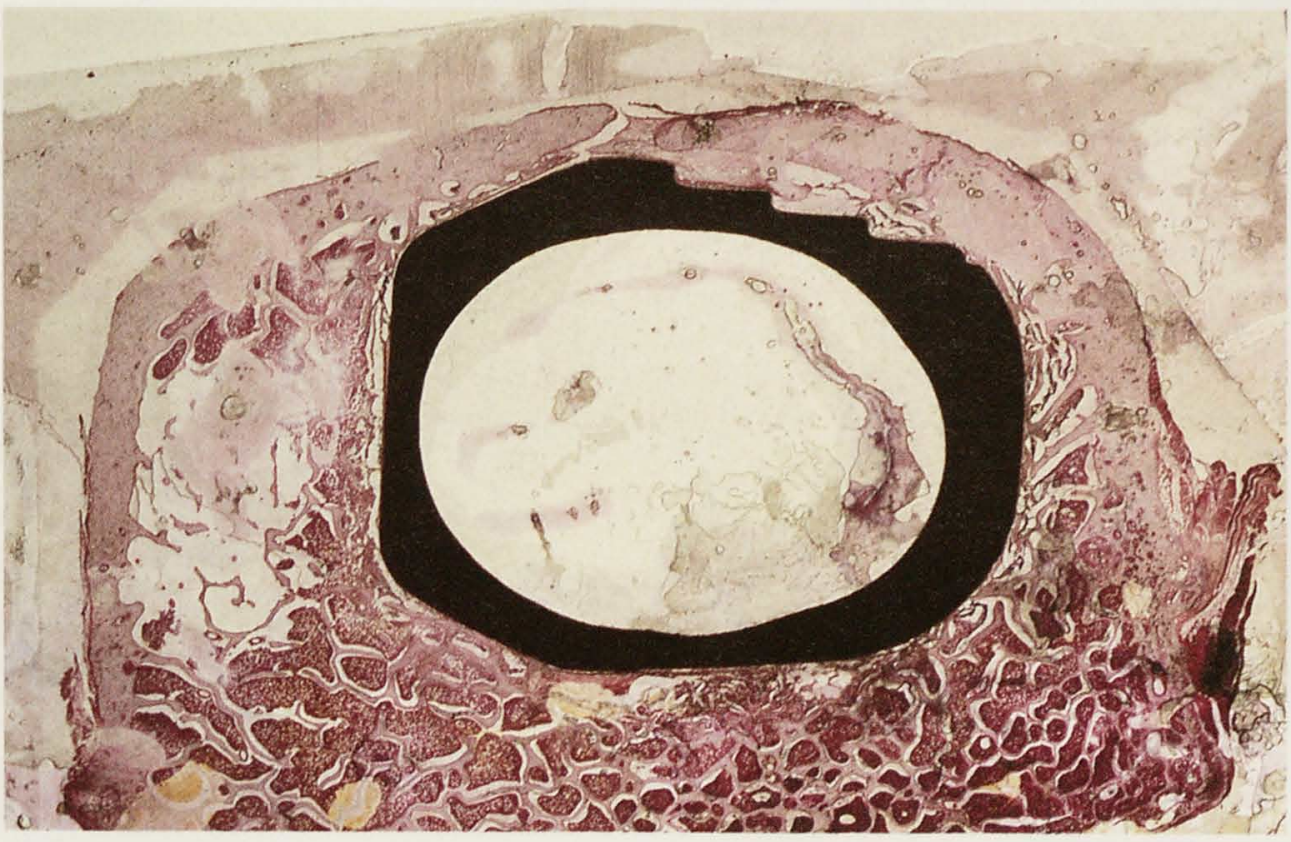

Fig. 69

Full bony envelope around hydroxyl-apatite coated femoral stem in proximal femur. (basic fuchsine, six weeks, $\mathrm{x} 1)$.

new bone formation around hydroxyl-apatite coated prostheses

Most bony defects, slits and spaces have closed by the three week interval in the proximal stem area (Fig. 75). At six weeks post-operatively, the coated implants are near one hundred percent covered with bone. Only around the femoral stem tip remains some fibrous tissue at the six week interval. The ossification in this area will take up to three months if bone is not too far away. Measurements of new bone formation can be made during the first three months after implantation, because old and new bone exhibit different staining properties; additionally, the use of fluorescent markers allows the identification of new bone. Defects up to a distance of $2 \mathrm{~mm}$. can bridge from neighboring bone. In the three and six week follow-up periods, the demarcation between young bone with a random orientation of bone trabeculae and the older mature bone is clearly visible, both in light-microscopy and fluorescence-lighting (Fig. 76). These pictures show that 


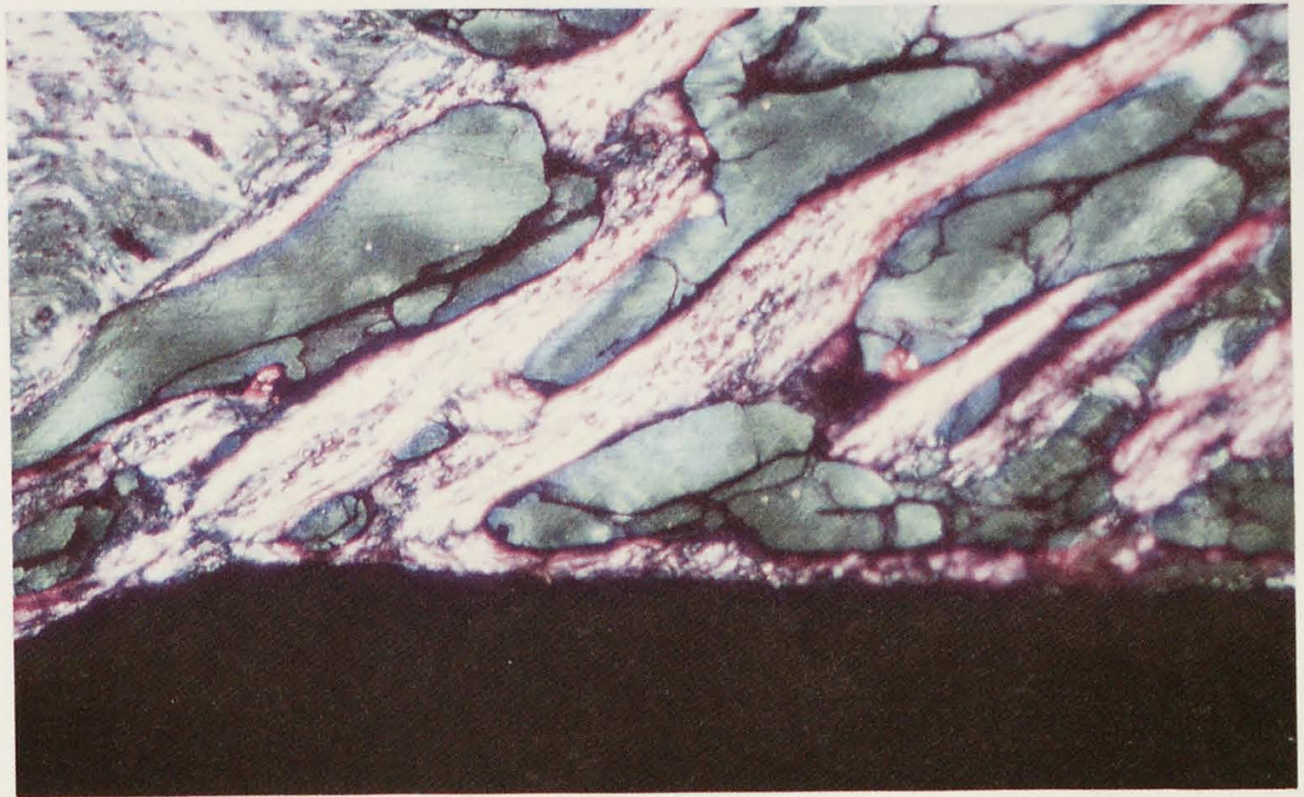

Fig. 70

Characteristic interface of hydroxyl-apatite coated femoral stem. Thin bone plate on implant surface with connecting trabeculae. Older pre-existent bone in upper left corner (Alcian-blue, three weeks, x100).

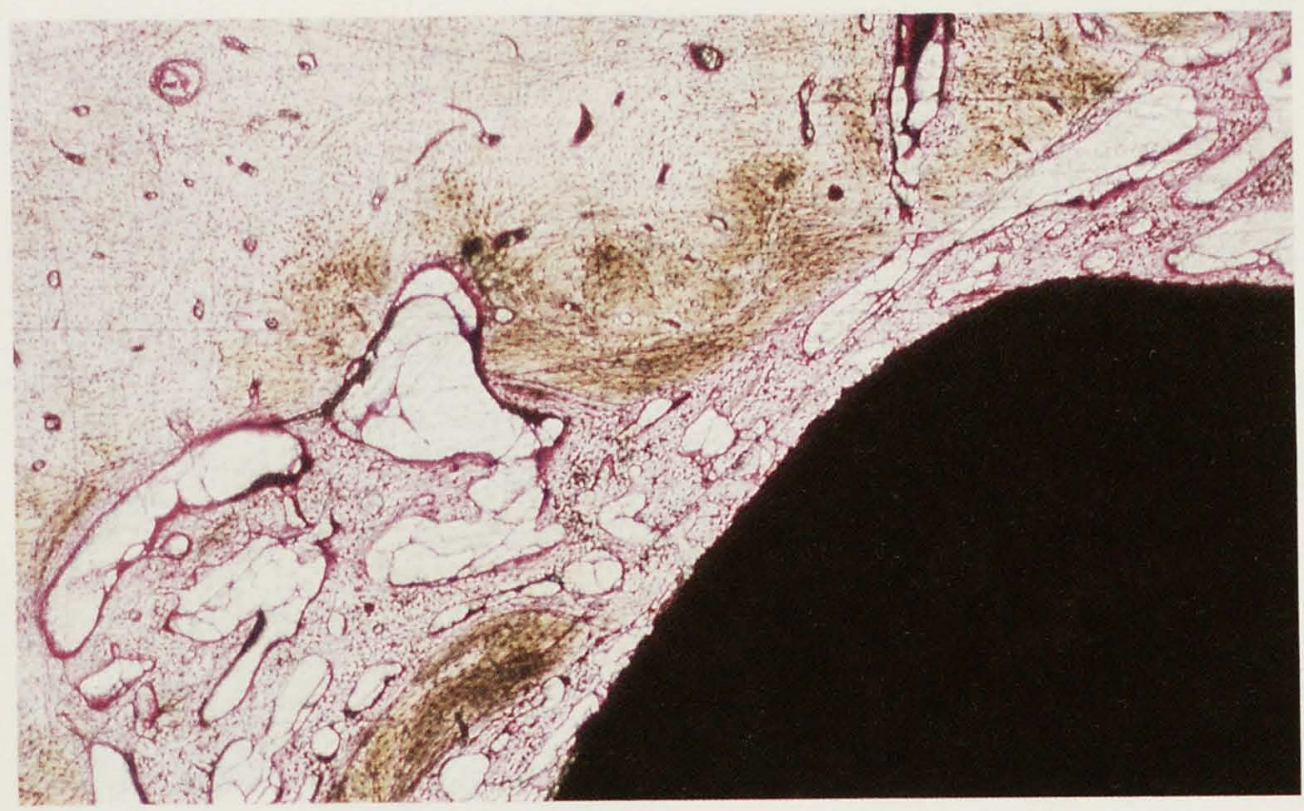

Fig. 71

Transition from old bone (brown) to newly formed bone (purple) in implant-bone interface of hydroxyl-apatite coated femoral stem (Alcian-blue, six weeks, x10). 


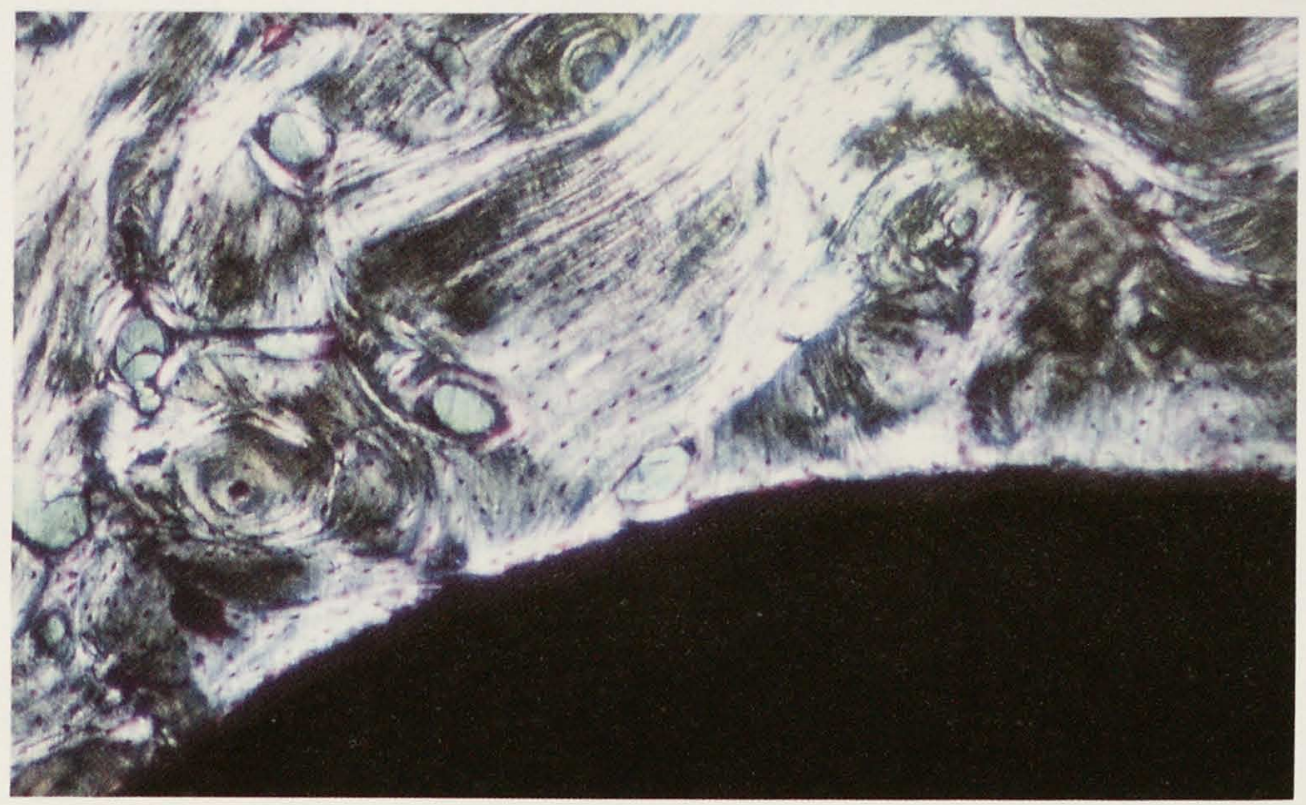

Fig. 72

Thick and dense bone structure around hydroxyl-apatite coated femoral stem at one year. (Alcian-blue, x10).

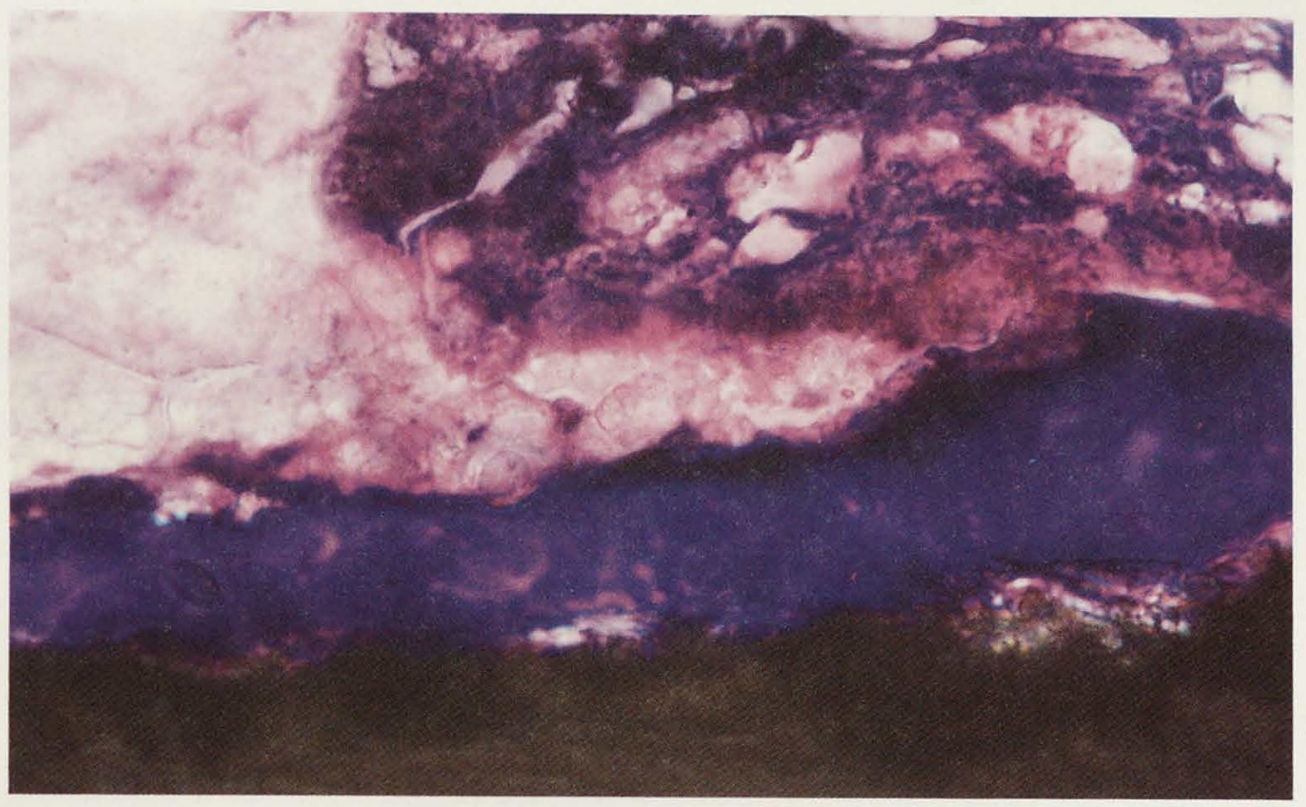

Fig. 73

Osteoid material deposition on hydroxyl-apatite coating (blue) by group of osteoblasts. (Alcian-blue, three months, $\mathrm{x} 500$ ). 
gap-filling and bone apposition around hydroxyl-apatite coated implants proceed from two fronts. The one is the usual healing phenomenon at the femoral side of the bone, were intra-medullary reaming has set the bone limit. Apposition of young bone on these older bone areas is seen without signs of bone necrosis or other intermediate adverse tissue effects. The other more unusual phenomenon is apposition of new bone on and gap-filling from the bone plate on the hydroxyl-apatite coating. Closure of any bony defects around coated implants therefore proceeds very fast. If there is no bone in close proximity to the coated implant, no new bone forms. At most there is a thin lining of bone apposition on the implant surface. This is probably derived from migrated osteocytes during the implantation stage of the prosthesis. The largest distance that can be bridged from neighboring areas with this process of gap-filling appears to be approximately 2-2.5 millimeters, as measured in the microscopical sections. This process is, amongst other factors, dependant upon the local geometry of the bone and the implant. Sharp gap angles with bony contact in the corners of the angle close easily, proceeding from this corner outward. More wide angled gap areas between implant and bone have a greater tendency to rounding of the bone in the corner area, the bone defect being filled to a lesser degree.

\section{bone-resorption around hydroxyl-apatite coated prostheses}

It is difficult to find any evidence of bone-resorptive processes in the direct vicinity of the implant-bone interface of hydroxyl-apatite coated prostheses. Nowhere is there any analogon visible to the bone-resorptive processes, seen around non-coated titanium implants at their implant-bone interface. There are however other phenomena, that play a role in bone resorption at the outer part of the femur around hydroxyl-apatite coated prostheses. Several implants showed more or less extensive loss of cortical thickness in the medial proximal femur, as sign of stress-shielding osteo-porosis. Two hydroxyl-apatite coated implants at the one year follow-up interval and one of the two at the two year followup showed mild loss of cortical thickness (fig. 46). One dog at the two year follow-up showed extensive loss of cortical thickness at the medial side of the proximal femur and spongialization of the cortical bone (fig. 54). These phenomena consist of increasing loss of cortical thickness and increasing cyst formation in the cortical bone. The initially small cysts in the cortical bone enlarge and, with continued remodelling, there is a gradual transition to almost trabecular appearance of the bone (fig. 79). At the two year follow-up interval; the one implant with extensive loss of cortical thickness in the proximal femur, did not show as much densification of trabecular bone in this area, as did the other implants at longer follow-up. There was however more extreme dense trabecular bone formation around the distal stem tip of the implant, thereby more unloading the proximal femur. At the distal level, there was only slight osteoporosis and spongialization of the femoral cortex.

Otherwise, there often is slight rounding of the resected edge of the femoral calcar, comparable in extent to the non-coated implants.

structure of bone with hydroxyl-apatite coated prostheses.

$I$ - medial proximal femoral area

Bone in the medial calcar area develops within three to six weeks a strong union with the implant surface. The bone trabeculae are rather thick and fully interconnected (Fig. 77). Near the implant surface they merge towards a thick bony endplate. The orientation of 


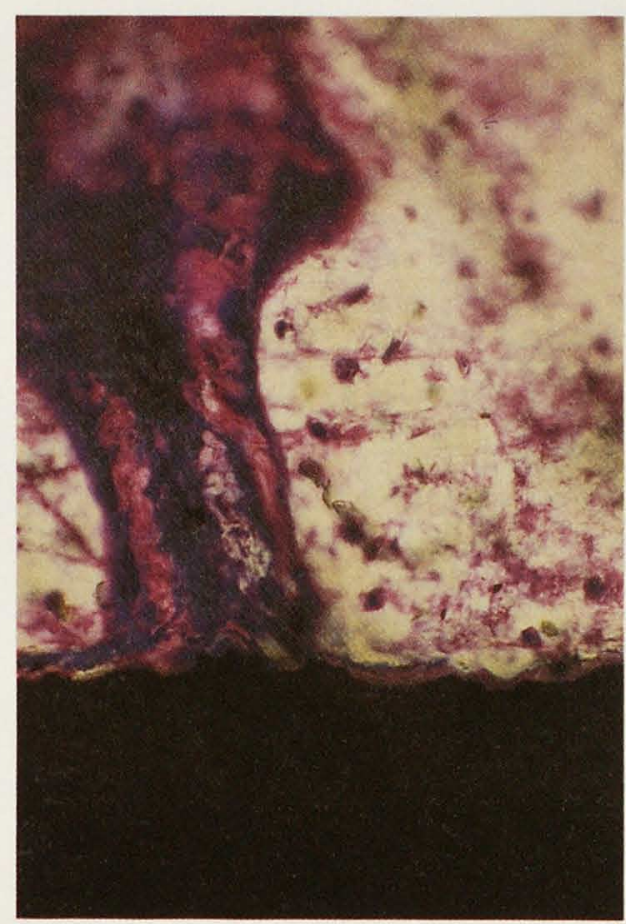

Fig. 74

Haversian system with ending on the hydroxylapatite coating (Alcian-blue, three months, x100).

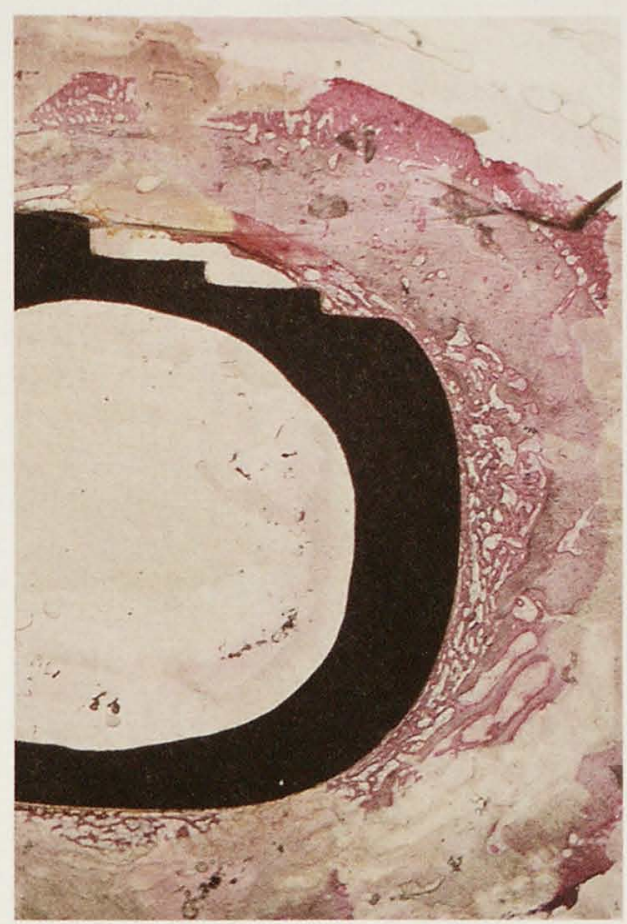

Fig. 75

Partial filling of bony defects around mid-stem of hydroxyl-apatite coated femoral component (basic fuchsine, three weeks, $\times 2$ ).

the bone trabeculae is at right angles to the implant surface and more oblique towards the anterior and posterior corners of the implant. With longer follow-up (three months), the bone trabeculae fuse together into a thick bone mass. Then, the direction and structure of the individual trabeculae can not be traced anymore. Near the one year interval, there is a strong bone plate on the medial implant surface, although there are some stress-shielding phenomena, consisting of spongialization or loss of cortical bone in this area. These were moderate at the one year follow-up interval and more severe at the two year interval in one of the two dogs (Fig. 78).

\section{2 - antero-medial and postero-medial calcar area}

Near the antero-medial and postero-medial parts of the calcar, the bone is dense. Newly formed bone is distinguishable from the older by its different staining properties in the first three months after implantation (compare fig. 76). Orientation of bone trabeculae in this initial period is mainly oblique to the implant surface in a lateral direction.

Frequently, old and new bone are completely intermingled. After three months of implantation this difference in staining properties disappears because of bone maturation and the bone mass further increases.

The stepped surface structures near the postero-medial implant surface show good filling 


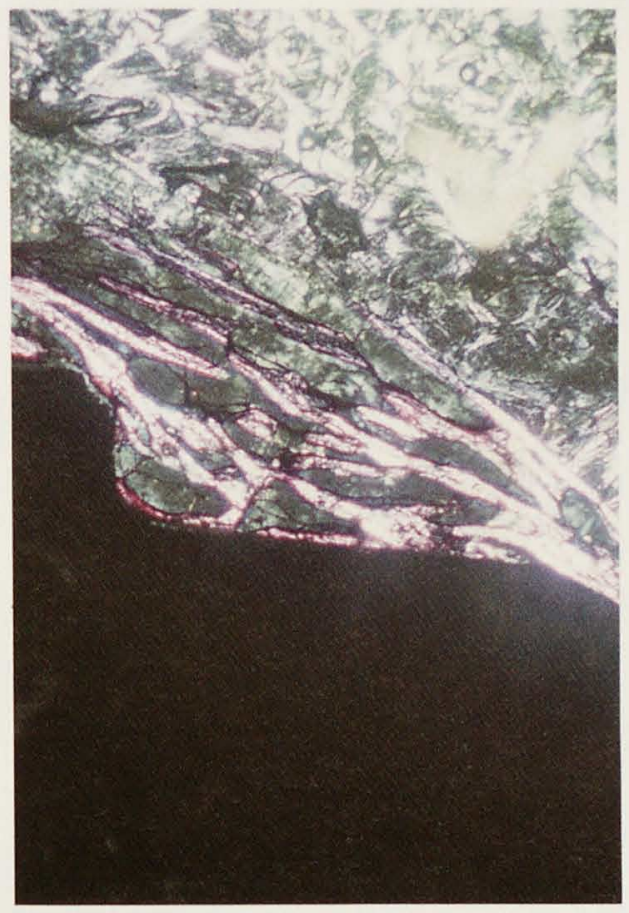

Fig. 76

Newly formed bone trabeculae stand out against the older bone in proximal femur around hydroxyl-

apatite coated stem. (fluorescence with polarization, three weeks, $\mathrm{x1}$ ).

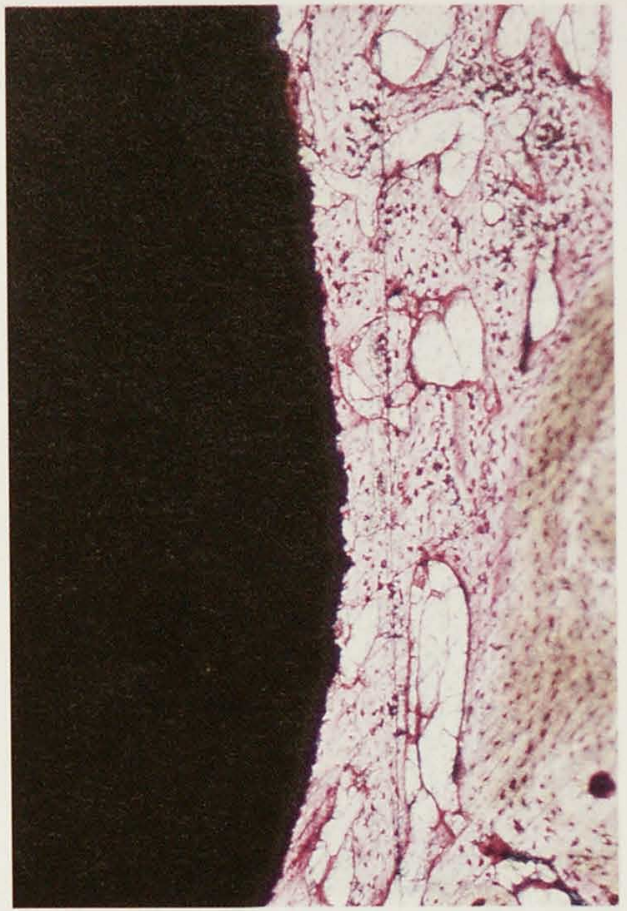

Fig. 77

Structure and orientation of bone-trabeculae at medial side of proximal femur (basic fuchsine, six weeks, $\mathrm{x} 10$ ).

with bone, starting within three weeks. The bone formation starts near the medial steps and proceeds from there towards the more laterally located ones.

At the six week interval also the more lateral steps have completely filled with dense bone.

\section{3 - anterior and posterior femoral area}

At the anterior and posterior sides of the prosthesis bone formation appears less rapid.

Nevertheless, complete bony union with a bone-plate on the implant surface, is obtained within the six week interval. Initially, the bone trabeculae are thinner as compared to the medial parts of the femur but ultimately a good bone density is achieved. There are stressshield phenomena, consisting of loss of cortical'thickness at several areas at the one or two year interval (Fig. 79).

\section{4 - lateral and trochanteric area}

In the lateral trochanteric region strong bone formation occurs within three weeks. The lateral corners especially constitute strong rims of primary bone contact. At the three week interval, there is good bone formation visible. The newly formed bone trabeculae are still thin and their orientation is at right or oblique angles to the bone plate on the implant surface. The endings towards the bone have a characteristic funnel-shape, spreading out as soon as they reach the implant surface (Fig. 80). After two years, bone density is high in 
this area, but the individual bone trabeculae and their orientation are no longer discernible in normal light microscopy. The cortical wall is grossly intact, without stress-shield phenomena in this area.

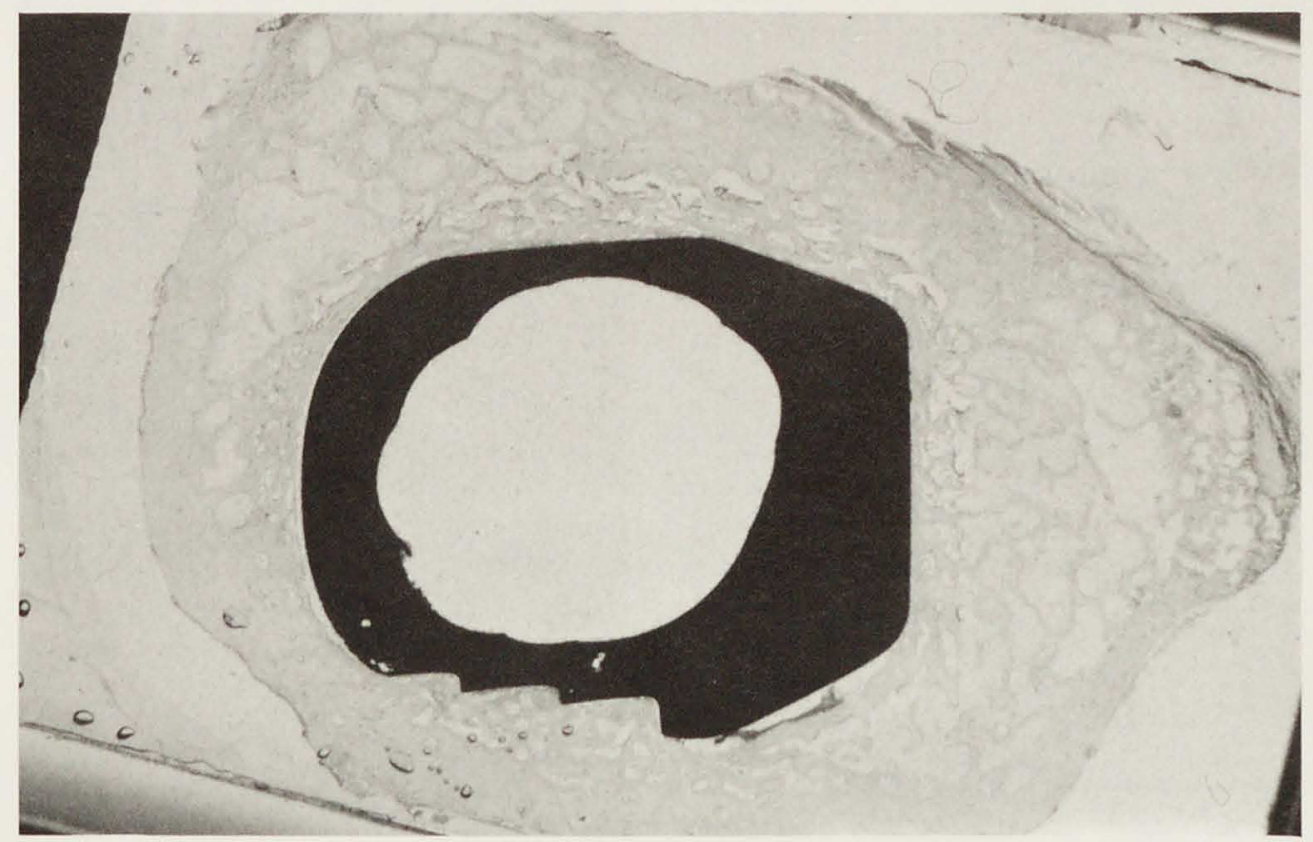

Fig. 78

Structure and orientation of bone-trabeculae of transverse section of proximal femur, preparation artifact at medial side. Femoral cortex preserved around mayor circumference of implant (basic fuchsine, two years, xl).

\section{bony defect filling around hydroxyl-apatite coated implants.}

In almost all areas around hydroxyl-apatite coated prostheses there is significant potential for closing bony defects, slits and spaces. Within three weeks of implantation of a hydroxyl-apatite coated implant, there is a bone plate present on the implant surface. Further filling of bony defects then proceeds from two ossification fronts. The one being from surrounding bone towards implant, the other being from implant-covering bone plate towards surrounding bone. This provides a considerable increase in speed for bony fixation and gap-filling. In the histological sections the acquisition of bone formation and union between implant and bone is advanced within three weeks and almost completed at six weeks.

Initially, the newly formed bone has a woven and lamellar structure, but with time takes on the appearance of dense cortical bone along most of its contact area with the implant. In the proximal and distal femoral stem area, the bone is of good quality and the trabecular structure is very dense when compared to the non-coated prostheses. In the mid-stem area bone density is lower, although even there, the bone density is higher as compared to the control side. In general, bone density close to the implant surface is much higher as compared to some distance away from the implant, for instance, halfway between prosthesis and surrounding cortical bone (compare fig. 78) 


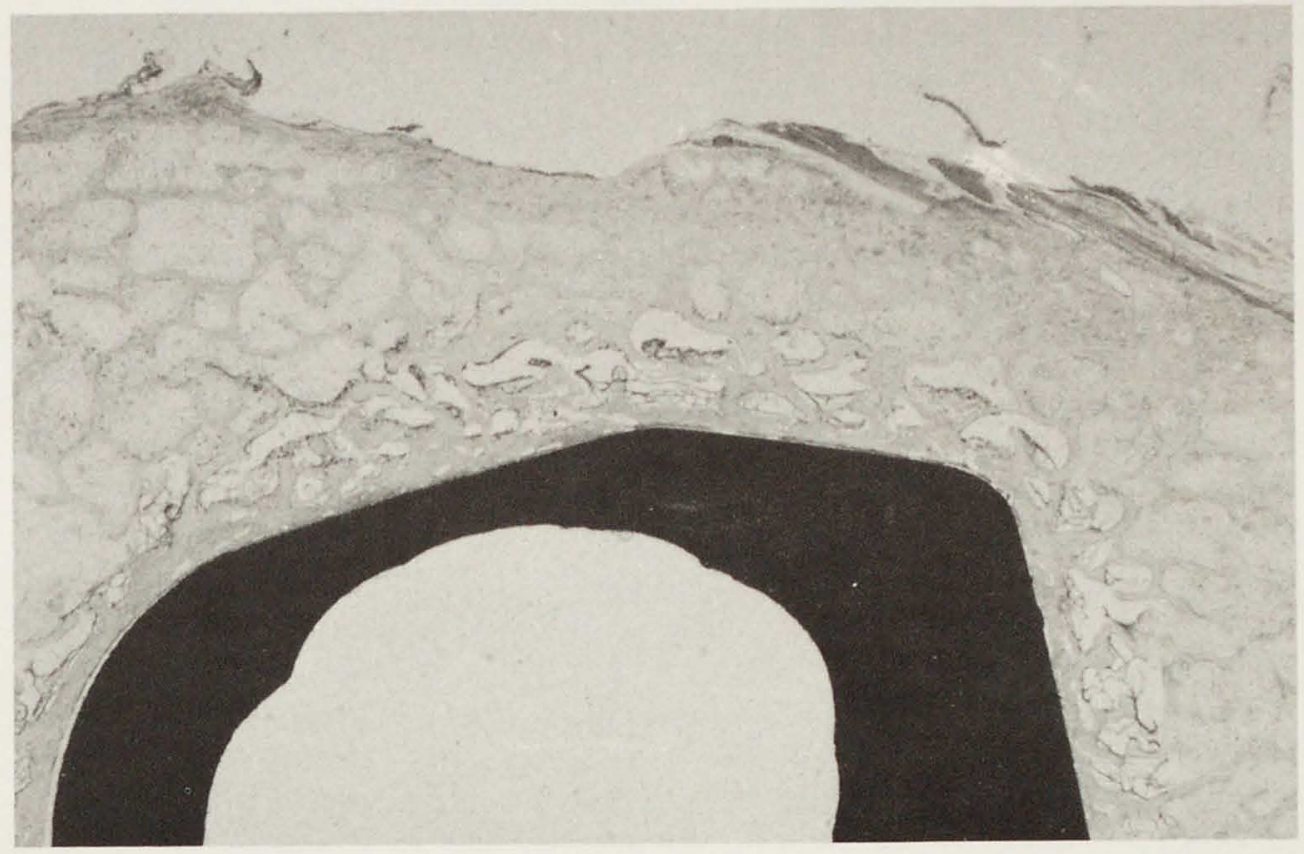

Fig. 79

Structure and orientation of bone-trabeculae at anterior side of proximal femur. Dense bone near implant surface. Observe stress-shielding effects: thinning of femoral cortex in upper left corner and spongialization of cortex in upper right corner. (basic fuchsine, two years, $\mathrm{x} 5$ ).

\section{differences in response with cortical or trabecular bone with hydroxyl-apatite coated} prostheses

As mentioned earlier, bone formation proceeds rather fast around hydroxyl-apatite coated implants. After one and two years, strong dense bone of cortical character is present both in the proximal and distal femoral stem area. The quality of surrounding cortical bone is thus maintained in the proximal femoral canal, while the trabecular bone originally present in the intra-medullary part of the distal femoral canal is replaced by a more dense quality of bone. The end result of these processes, as far as bone density is concerned, is the same for both areas. Consequently, there appears no difference in response of hydroxyl-apatite coatings with cortical or trabecular bone. This means that other factors than these are responsible for these processes.

\section{condition of hydroxyl-apatite coating}

In microscopical sections after one and two years, the hydroxyl-apatite coating is clearly discernible.

It has a smooth, dense appearance with a sharp transition from coating to bone. There is no fragmentation of particles. The thickness of the coating can be determined with the aid of a micrometer. A remaining thickness of 10-30 microns can regularly be observed. The recorded values however, are not very precise, because there is no guarantee of the measurements being made perpendicular the implant surface. Also, initial coating 
thickness of the implants was somewhat variable and not precisely known for each location. Some degradation of the hydroxyl-apatite coating can therefore not be excluded, and has probably indeed occurred. It is known that in the first few months after implantation, during acquisition of bone-bonding, some superficial degradation of the hydroxyl-apatite coating occurs, because of the increased surface porosity of the outer layer of the coating. After two years follow-up, the remaining coating thickness appears more than adequate to insure implant fixation. Remaining coating thickness in itself is not very critical, because bonding to hydroxyl-apatite coatings is a surface phenomenon and not dependant upon any depth of ingrowth into the coating that has a closed surface and dense structure of its deep layer. Longer follow-up than current two years are necessary to establish the long term stability of hydroxyl-apatite coatings. After two years, polarization microscopy reveals the direction of individual bone collagen-fiber-systems to the implant surface (Fig. 81 and 82 ). The ending angles are rather variable, varying between perpendicular and oblique, true parallel endings are seldom seen.

\section{Response of bone-marrow with hydroxyl-apatite coated prostheses}

The bone-marrow in the proximal femur has largely been reamed away. Most of the bonemarrow has to regenerate from remaining cell lines. In the first three to six weeks after implantation, there is a predominant inflammatory response of the regenerated bonemarrow with polymorpho-nuclear leukocytes. After three to six months, this inflammatory response ceases and mainly lymphocytes and plasma-cells are present (Fig. 83). The pictures are similar to those of the non-coated titanium implants.

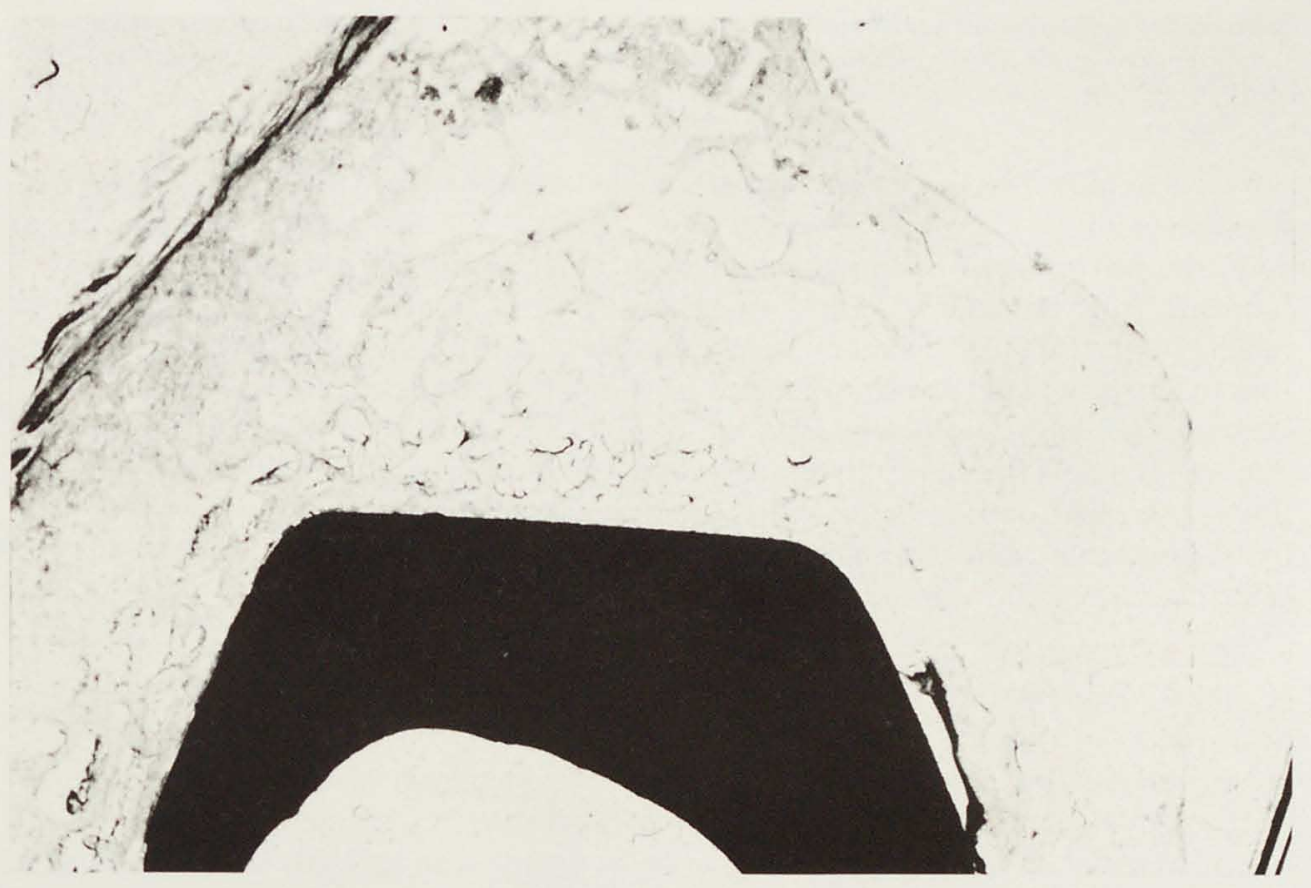

Fig. 80

Structure and orientation of bone-trabeculae at lateral side of proximal femur (basic fuchsine, two years, $\mathrm{x} 5$ ). 


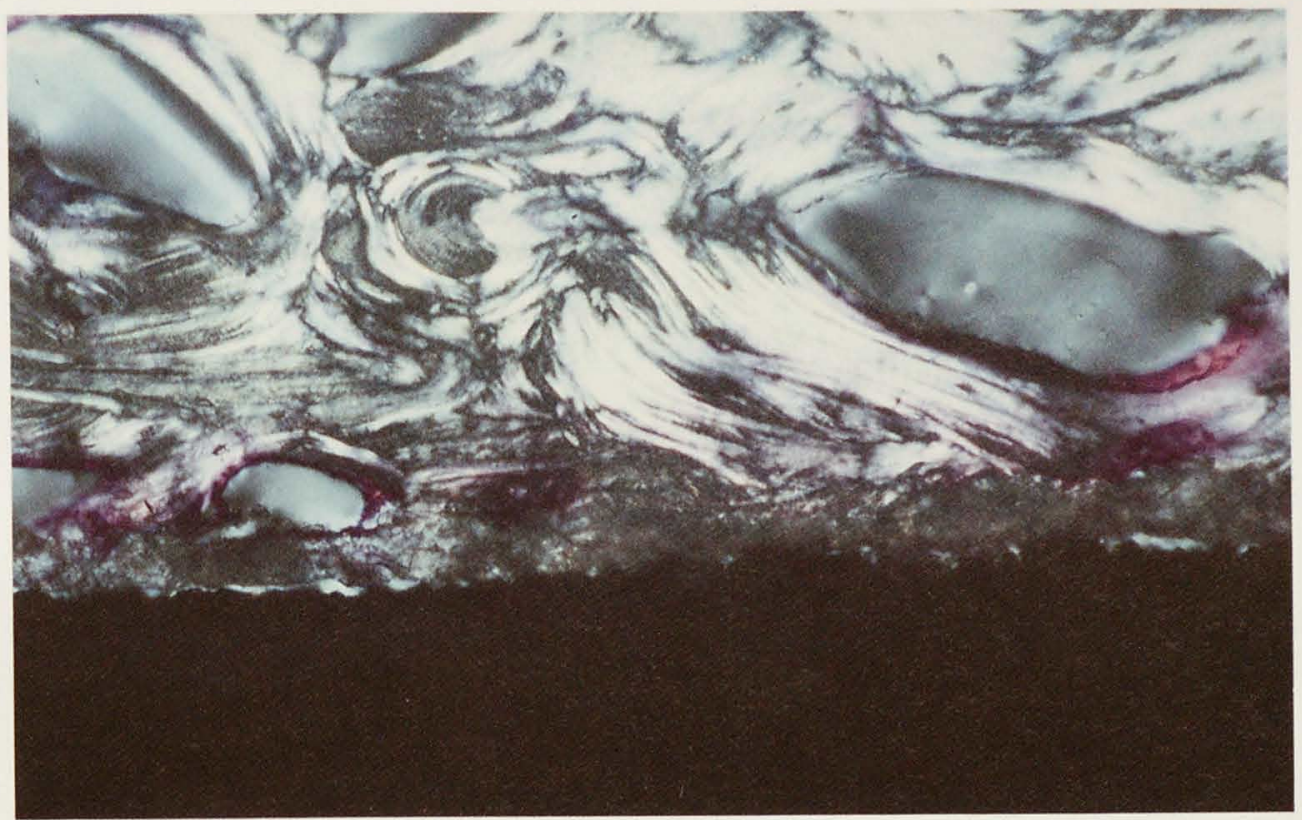

Fig. 81

Density of bone and structure of hydroxyl-apatite coating at two years. (polarization, x50).

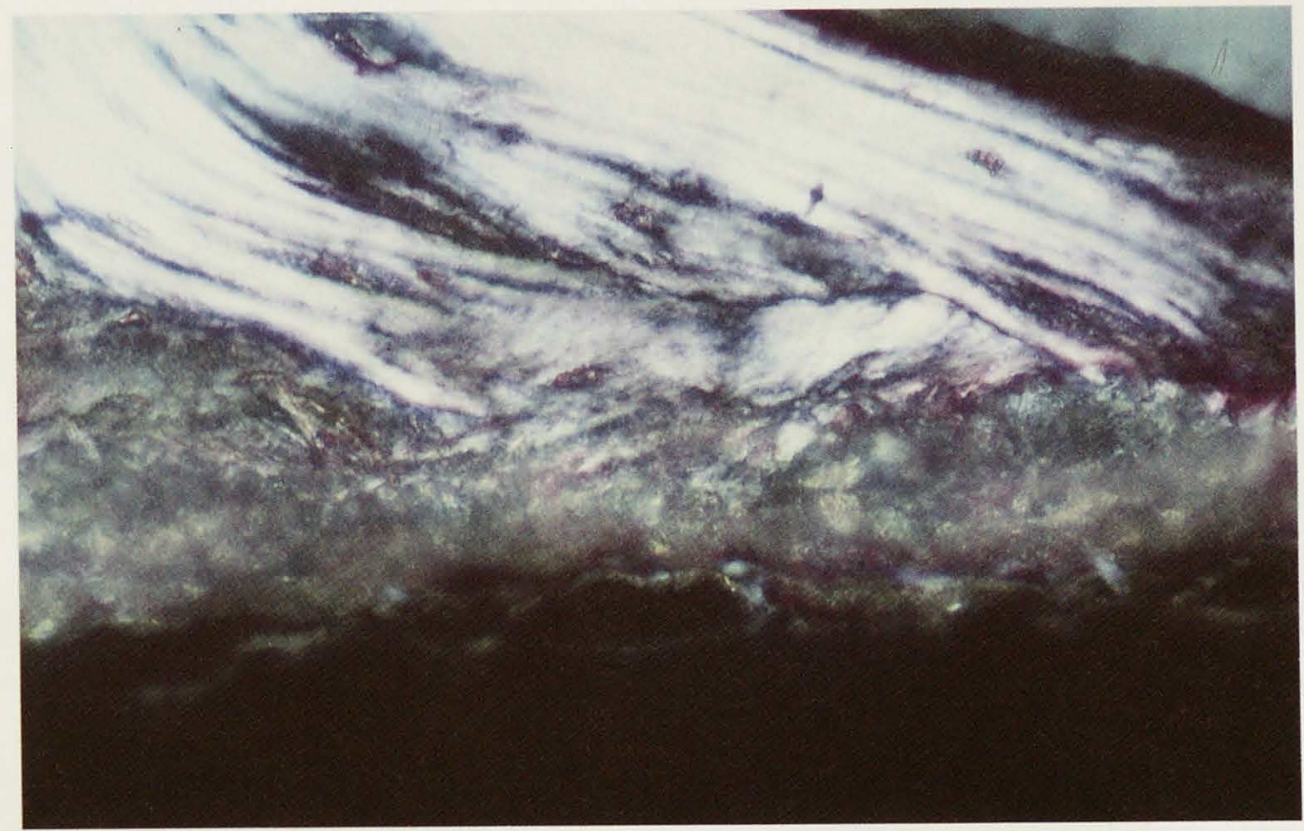

Fig. 82

Structure of coating and orientation of bone fibers against hydroxyl-apatite coating at two years. (polarization, $\mathrm{x} 500)$. 


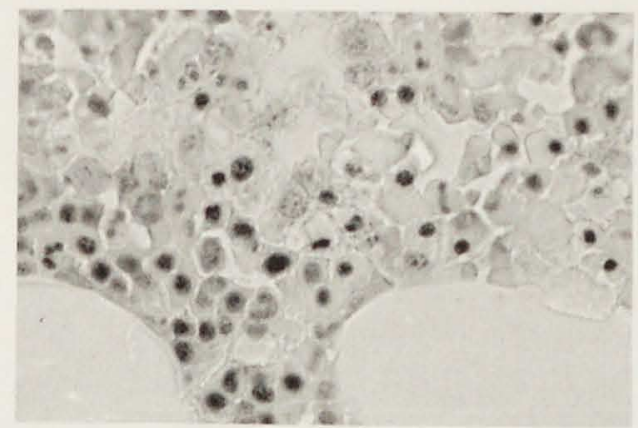

Fig. 83

Plasma-cells and lymphocytes constitute the majority of cell lines in bone-marrow distal to hydroxyl-apatite coated femoral component (HE, six months, $\mathrm{x} 600$ ).

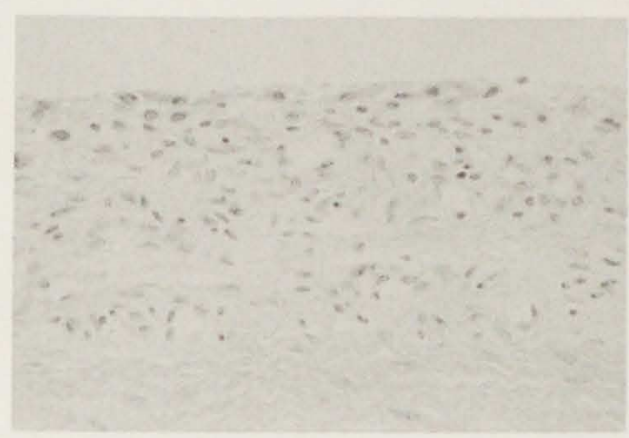

Fig. 84

Fibroblastic response of prosthetic neo-capsule. Multicellular layer of fibroblasts in neo-capsule of hydroxyl-apatite coated canine total hip replacement. (HE, three weeks, $\mathrm{x} 500$ ).

\section{Response of joint neo-capsule with hydroxyl-apatite coated prostheses}

The prosthetic neo-capsule has the normal appearance of fibrous tissue. In the first three to six weeks, the superficial layer of fibrous tissue, in contact with the prosthetic collar, contains multiple cell-layers (Fig. 84).

The fibroblastic response of the superficial layer ceases with time and after six months the lining layer has a thickness of only a few cells (Fig. 85). There are no pathological inclusions, giant-cell or macrophage reactions nor other adverse tissue reactions visible in the prosthetic neo-capsule. Para-articular calcifications were scarcely encountered, but were not significantly different in number or extent from the control group of non-coated implants.

\section{Discussion}

Results indicate that hydroxyl-apatite coated prostheses form very tight bonds with living bone. Despite their conical non-retentive shape and smooth surface, extraction from the femur is not easy. Powerful extraction after $3-6$ weeks of implantation or longer caused fissuring and fracturing. Radiology shows excellent bone quality with condensation of endosteal bone around hydroxyl-apatite coated implants at their implant-bone interface. Bone scintigraphy reveals an even distribution of $99 \mathrm{~m}$-technetium uptake over the entire coating area without hotspots or other signs pointing towards instability at the implantbone interface. The histology confirms the very close bonding of hydroxyl-apatite coating with bone. Not only the mature osteocytes have good bonding with the hydroxyl-apatite coating, but also the young osteoblasts. The regenerated bone-marrow has a quiet appearance with predominant population of lymphocytes and plasma-cells. The prosthetic neo-capsule does not show any abnormal reactions, nor abnormal calcifications. This could theoretically be conceived with a bone-related material like hydroxyl-apatite.

The femoral bones around the coated prostheses did show evidence of bone resorption by stress-shield phenomena. In the first half year of follow-up, some rounding of the resected calcar edge was regularly seen, explainable by the different way of stress transfer in natural hip joints and implanted prostheses. With longer follow-up more extreme stress-shield- 


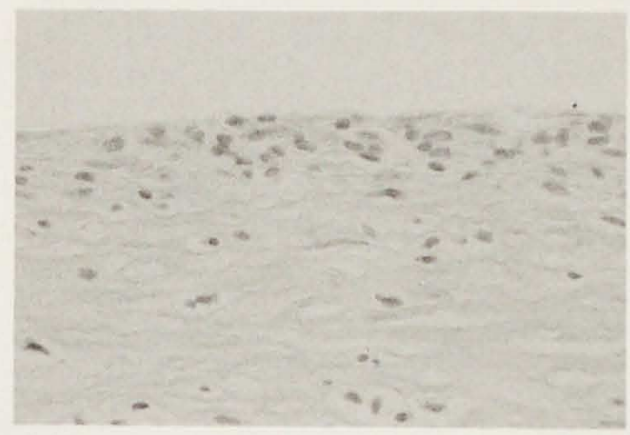

Fig. 85

Thin layer of fibroblasts in neo-capsule of hydroxylapatite coated canine total hip replacement. (HE, one year, x500).

phenomena were sometimes observed in the medial proximal femoral cortex. There seemed to be link with the amount of trabecular bone densification observed around the coated implants. One dog, at the two year follow-up interval, showed extensive loss of cortical thickness in the proximal femur, with transition of cortical bone to almost trabecular bone. The normally observed densification of trabecular bone at the implantbone interface, was not very well developed in the proximal femur, but was indeed the more extensive around the distal tip of the femoral stem. The other implant at the two year interval and both dogs at the one year interval did not show that extensive loss of cortical thickness. This case, there was the more regularly observed increase of trabecular bone density, equally well distributed over proximal and distal stem section of implants. It is thus conceivable, that the loss of cortical thickness in the proximal femur has a relation with the amount of distal fixation of implants. The more rigid the distal fixation of the stem tip, with unloading of the proximal femur, the more the bone in the proximal femur is prone to stress-shielding osteoporosis. Amoung others, one causative factor was probably the full length of hydroxyl-apatite coating on the stem tip. Otherwise, especially the femoral cortex around the hydroxyl-apatite coated implants was of reasonable good quality without excessive signs of spongialization or lysis of bone. However, also the noncoated implants suffered from stress-shield phenomena, although at a lesser rate as compared to the hydroxyl-apatite coated implants.

In this study, the non-coated prostheses showed radiological and scintigraphic signs of instability at the implant-bone interface. Histology shows fibrous tissue at their bone interface. Although this might be in agreement with the now somewhat obsolete philosophy of "pressfit" fixation by fibrous-tissue interposition, it is certainly not in agreement with the concept of osseo-integration of titanium implants. In contrast, the hydroxyl-apatite coated prostheses show a strong bony interface. Directly after implantation the bony architecture around coated and non-coated implants is the same. Both have the same pressfit fixation in the proximal femur, with an initial surface contact area between bone and implant of approximately twenty percent. Gaps between implant and bone can be as wide as $2 \mathrm{~mm}$., as determined in microscopical sections. 
The persistent lack of adherence between the titanium surface of the non-coated implant and bone results in continuing micromotion at the interface. This occurs even at relative minor stress levels and can be ascribed to the difference in mechanical characteristics of bone and metal, mainly their stiffness. Micromotion is evidenced by both the radiographic, scintigraphic and histological findings. There are radio-lucent lines on the radiographs pointing towards bone resorption, which may cause micro-motion of the implant. Bone scintigraphy reveals a three point hot spot with accumulation of radionuclide, characteristic of micro-instability of the implant. The histology shows a fibrous tissue interface, which can not provide sufficient mechanical strength for hard tissue stabilization.

Persistent micro-motion at the bone interface is an established cause of bone resorption (Perren 1983). This bone resorption again increases (micro)-motion and thereby maintains, in a vicious circle, the fibrous tissue nature of the interface. Although the bone shows an osteoblastic response in response to this bone resorption, as evidenced by bone scintigraphy, the net result is zero because this bone formation is just enough to compensate the bone losses by the resorptive processes. Even after two years the equilibrium between these processes of bone formation and bone resorption appears delicate. It could easily shift to one or other side depending probably on the mechanical loading conditions of the bone, and thereby on the amount of micromotion in the implantbone interface. One interesting observation concerns the phenomenon of radio-dense lines peripheral to the radio-lucent lines around non-coated prostheses, as seen on radiographs. In the histological sections of non-coated prostheses, there is no parallel to this phenomenon. What does occur, is a parallel orientation of bone trabeculae peripheral to the radio-lucent line.

Because other bone areas around non-coated prostheses have a more or less random orientation of bone trabeculae, the net result on the radiograph is one of radio-dense line formation, because the whole of calcified mass in this direction of projection is much higher as compared to other directions.

With hydroxyl-apatite coated implants there is initially an equally large surface contact area with the surrounding bone.

In the first few weeks after implantation, there is always a relative unloading of the extremity, caused by postoperative pain and discomfort. With dogs, this period is only a few weeks in duration. Even this relatively short period is long enough for rigid bony fixation to develop in the areas with primary bone contact. This process of early primary bone-bonding effectively eliminates micro-motion at the implant interface once joint loading commences. Afterwards, even empty areas caused by slits and spaces around the implant can ossify by processes of secondary filling with bone. Even this may proceed rather fast, because filling of bony defects between bone and hydroxyl-apatite coatings proceeds from both implant surface and bone contact area. This is a major difference with conventional implant materials, that can at best exhibit an ingrowth from bone towards implant surface, but never in the reverse direction.

In the histological sections, ossification is well advanced within three weeks and almost complete within six weeks, at least in the important weight-bearing areas of the implant. Only with large bony defects more time is needed for full ossification. At three months, an 
equilibrium is present for bone formation. Further progress of the zone of ossification is not likely after this period. Distances of up to $2 \mathrm{~mm}$ can fill this way from neighboring bone, as measured in the microscopical sections. There is no bone-inductive influence from the hydroxyl-apatite material itself. If no bone is present within short reach no new bone will regenerate.

Although it is very difficult to quantify exactly remodelling phenomena in bone, there were some interesting observations concerning the orientation of the bone trabeculae around the hydroxyl-apatite coated implants. Along the medial calcar area the bone trabeculae fuse to a thick bony endplate with gross orientation at right angles to the implant surface. The bone trabeculae are thicker close to the implant surface as compared to some distance away from the hydroxyl-apatite coated implant. According to "Wolf's law" of bone architecture, this can be interpreted as orientation of bone trabeculae following local stress patterns. Additionally, the funnel-shaped endings of bone trabeculae on the implant surface, the increase in calcium content towards the implant surface and the perpendicular or oblique orientation of individual bone collagen-fiber-systems on the hydroxyl-apatite coating accentuate the load-carrying characteristics of hydroxyl-apatite coatings and suggest that the bone in close proximity to the hydroxyl-apatite coating takes part in the process of remodelling. These remodelling phenomena are further examplified by the signs of stress-shielding in the proximal femur. If there is rigid distal fixation around the femoral stem tip, there are signs of loss of cortical thickness in the proximal femur. On the contrary, if there is also good densification of trabecular bone around the proximal stem part at the implant-bone interface, stress-shield phenomena are much less extensive. Both implant geometry and the full area of hydroxyl-apatite coating play a significant role in this process of stress-shielding osteo-porosis. It is probable, that a more restricted proximal area of hydroxyl-apatite coating would have caused less extensive loss of cortical thickness in the proximal stem area.

When a prosthesis is capable of transferring shear and tensile forces this also means that compressive stress on the bone is drastically reduced under the same loading conditions. A conventional prosthesis can only transfer compressive forces by lack of adherence between implant and bone. Because shear and tensile forces will occur anyway in the bone, these forces are converted to additional compressive forces.

Bone scintigraphy, using $99 \mathrm{~m}$-technetium, reveals that bone metabolism around hydroxyl-apatite coated implants is very even, after the initial period of bony ingrowth. Around the non-coated implants there is a persisting three point hot spot accumulation of technetium up to two years after surgery, indicative of persistent local increase in bone metabolism. The primary cause is micro-instability of the implant that induces bone resorption because it overcharges the compressive resistance of the bone. Although this bone resorption is partially balanced by increased bone proliferation, (the hot spots on the bone scan), the net result is not sufficient to provide adequate skeletal stabilization of noncoated prostheses.

In contrast, the hydroxyl-apatite coated implants show a very even distribution of technetium uptake. It has a near linear relationship to the calcified bone mass over the entire area of the femur in contact with the hydroxyl-apatite coating. This is a sign of 
relative stabilization of bone metabolism at the implant-bone interface. It points towards good bony integration of the implant after the initial period of bony ingrowth, without signs of persistent instability at the implant bone interface. The conclusion from the bone scintigraphy is that hydroxyl-apatite coatings add to a more physiological bone metabolism around implants and that such implants are better integrated into the bone than their non-coated counterparts.

The bone bonding properties represent one major advantage of hydroxyl-apatite coatings over conventional bioinert implant materials. It could well be, that a second major advantage of hydroxyl-apatite coatings on implant materials constitute their osteoconductive properties, which means they provide the bone a scaffold for ingrowth in the shortest possible time. As mentioned earlier, surgical implantation of a cementless hip implant provides, even with accurate instrumentation, an initial bone contact area of less than 20 percent. From experimental studies (Carlsson ea. 1988, Harris ea. 1983), it is known that implant-bone gaps as small as $0.35-0.5 \mathrm{~mm}$ are never bridged by cortical bone. Instead, these gaps are filled by fibrous tissue. Other authors (Albrektsson ea. 1987) report more favorable characteristics, especially with titanium, but even then, a prolonged period of unloading of the implant is necessary to accomplish appropriate bone healing or "osseointegration". Only under conditions of complete unloading can bone grow up to bioinert titanium implant surfaces and thereby increase the initially small contact area between implant and bone. At the same time prolonged unloading of bones is contradictory to the maintenance of good bone stock. This will lead to disuse osteoporosis of bones. Under conditions of heavy joint-loading, for example, in the current study the bioinert titanium control implant group, histological failure of the implant-bone interface is the rule. There is a complete fibrous-tissue interface, in place of the desired "osseointegration".

Under high joint-loading conditions, histological failure was induced with the non-coated prostheses. Although clinical performance of the non-coated implants was not extraordinarily poor, the radiological, scintigraphic, and histological data indicate that the limits of tissue tolerance were exceeded. Micro-motion at the implant-bone interface, causing bone resorption, is certainly one factor. Also, the less favorable biological surface characteristics of the bare titanium implant can contribute to same. Results with noncoated prostheses are thus far inferior to hydroxyl-apatite coated prostheses at the tissue level. Although from a clinical point of view "pressfit" fixation through fibrous tissue interposition is feasible, we must realize that the theoretical foundation of this concept is not too well established. Stronger tissues than fibrous tissue are needed to cope with the high implant-bone interface stresses in a more physiological way.

Hydroxyl-apatite coatings permit a fast and reliable filling of bony slits and spaces that occur around cementless implants. This increases bony contact area between implant and bone from the original twenty percent, or lower, to a near one hundred percent. Even in the absence of any bone bonding properties of hydroxyl-apatite coatings, this would decrease the relative interface-stress of the implant with a factor of at least five. When the prosthesis is near one hundred percent covered with dense strong bone, micromotion is largely prevented and the resultant interface stress will be further reduced. Both radiological, scintigraphic, and histological findings with hydroxyl-apatite coated 
prostheses point towards a stable equilibrium at the implant-bone interface.

This phenomenon is, to some extent, comparable to the distribution of stresses through cemented hip joints. Although acrylic bone cement in itself is not exceptionally strong, it is weaker than either bone or implant metal, the overall mechanical performance can still be adequate, because acrylic bone cement allows a very good redistribution of loadbearing forces over its entire interface area.

Hydroxyl-apatite coatings can establish a comparable, complete support of the implant with bone, thereby distributing the loading-forces over a significantly larger surface area as compared to non-coated cementless implants. The reactive densification of bone around hydroxyl-apatite coated implants can probably be explained as an adaptation of the bone modulus of elasticity to that of the more stiff implant material. Both endosteal, cortical and trabecular bone exhibit this phenomenon. The character and amount of implant loading appear to be of more importance in this respect than the original characteristics of the bone. We must realize, however, that some bone must be present near the hydroxyl-apatite material for these bone-proliferative responses to occur. The critical distance appears to be approximately 2 millimeters, as measured in microscopical sections during the first three months after surgery. Hydroxyl-apatite has very good osteoconductive properties, but no osteo-genetic potential. If no bone is present within short distance, no new bone will form.

Especially with regard to osteo-conductivity, there is a marked difference with other bioinert implant materials. Using porous metal coated acetabular components in the dog, some authors (Carlsson ea. 1987, Harris ea. 1982) could demonstrate that gap areas as small as $0.35 \mathrm{~mm}$. are not filled by bone, but instead are bridged by fibrous tissue. In a study (Turner ea. 1986) comparing three different types of porous metal coatings, circumferential or partially coated plasma flame-spray, sintered bead or fiber-metal coatings, the authors report less ingrowth with the beaded surface at six months $(25 \%)$ as compared to the fiber-metal surface (37\%). In all groups, the ingrowth was incomplete, the proximal and distal areas of the implant showing higher rates of ingrowth as compared to the mid-section of the femoral stem. There was in general a proximal to distal gradient of loss of cortical bone at the (longest) six months interval, as sign of bone loss by stressshielding. The magnitude of loss of cortical bone was more dependant on the extent than the type of porous coating.

As compared to these results, hydroxyl-apatite coated implants permit a more complete coverage with bone in a shorter period of time. Even after six weeks, hydroxyl-apatite coated implants have a near one hundred percent coverage of bone around their surfaces. It is conceivable, although not yet proven, that the more complete bony integration of hydroxyl-apatite coated implants as compared to porous-metal coated implants, protects the implants better from stress-shielding phenomena. Stress-shield phenomena were observed with both the non-coated and the hydroxyl-apatite coated implants. The extent of loss of cortical thickness was related to the amount of distal fixation of the implants. Nevertheless, the extreme complete loss of cortical thickness with spontaneous perforation of porous-metal coated implants into the soft tissues of the femur at six months follow-up in their study (Turner ea. 1986), was not even observed in the hydroxyl-apatite coated implants of our study at two years follow-up. From both our study and that of 
Turner, the conclusion can be drawn that it is important to confine, and indeed enforce, load-transfer through implants to the proximal femur by applying coatings only to the proximal stem of the implant.

Because our work (Geesink ea. 1988 \& 1987, de Groot ea. 1988) was the first to be published on biological characteristics of hydroxyl-apatite coated implants, there are no comparable reports in the literature. Since 1986, there have been some congress presentations on the subject of hydroxyl-apatite coatings (Kay ea. 1987, Cook ea. 1986, Kent ea. 1986). Findings in their experimental studies, with plug-, intra-medullary implants and dental implants respectively, conform to our results. They also found good bone bonding properties with hydroxyl-apatite coated implants, although figures and numbers vary because of differences in experimental setup.

The concept of biological implant fixation to bone by using hydroxyl-apatite coatings on titanium substrates has proved successful under the heavy load-bearing conditions of this animal model. The original objectives of the study have been confirmed. The hydroxylapatite coating proves to be durable and strong without the fatigue failure problems of sintered hydroxyl-apatite. At the same time, hydroxyl-apatite coatings provide the same excellent bone bonding properties and biocompatibility characteristics of sintered hydroxyl-apatite with the added mechanical strength of metallic implants. Especially the short time interval in which a hydroxyl-apatite coating can achieve a strong bone bond and the fact that close apposition between bone and coating at surgery is not necessary to achieve good results are striking advantages over more conventional implant materials. Adverse biological effects have not been detected, except for the proximal stress-shield phenomena, that were more related to the extent of the coating on the stem than to its nature.

The general conclusion from this animal study is, that the composite material consisting of a hydroxyl-apatite coating on a titanium substrate is a good material for load-bearing applications in orthopaedic surgery. Because there were no adverse biological effects, the chemical bone-bonding concept using hydroxyl-apatite coatings appears safe for human applications. 


\section{Chapter eight}

\section{Infection in canine hydroxyl-apatite coated hip arthroplasty}

In the experimental study with canine hydroxyl-apatite coated total hip prostheses, discussed in a previous chapter, one of the dogs acquired a deep infection of the hip joint. After a luxation of the femoral component requiring open reduction three days after primary surgery, deep infection became apparent approximately three months later. There was a fistula over the joint and the discharge grew a mixed bacterial population of staphylococcus aureus and proteus. Obvious complaints by change in animal behaviour were absent, so treatment was expectative. At sacrifice of the dog, six months after primary surgery, there was still manifest discharge from the wound. This dog was excluded from the standard series of follow-up, but the findings at sacrifice were of enough interest to be detailed in this chapter. The behaviour of hydroxyl-apatite coatings under infectious conditions is not yet known. Although this is an incidental observation, the results of which can not be generalized until more data are available on same, the findings are still of clinical relevance, because they tend to suggest that the theoretical behaviour of hydroxyl-apatite coatings under infectious conditions also occurs in practice.

Theoretically, the hydroxyl-apatite coating is chemically unstable at a $\mathrm{pH}$-level of five or less. This means that in a slightly acid environment the hydroxyl-apatite coating will dissolve. Under infectious conditions, there is a lowered local tissue $\mathrm{pH}$-level, often five or below. So theoretically, the hydroxyl-apatite coating could start to dissolve under infectious circumstances and the implant could become loose after a while. To observe these phenomena, the dog was observed for some time after manifestation of the infection. The results are discussed below:

\section{Materials and methods}

The methods of investigation include the techniques for mechanical, radiological and histological evaluation, as detailed in the previous chapter.

\section{Results}

Visual inspection and mechanical evaluation

Dissection of the hip joint at time of sacrifice revealed a fistula, continuous to the femoral and acetabular component. The cemented cup was loose and could be removed without difficulty. The femoral component could not be extracted by manual force at time of dissection.

\section{Radiological evaluation}

Explant radiography of the femur showed signs of periosteal new bone formation, mainly in the proximal part of the femur (Fig. 86 and 87). There were small cysts visible in the bone, but no gross radiolucencies or scalloping around the implant. There were no radiolucent lines visible, nor radiodense lines peripheral to the implant. Bone quality was good when compared to the contra-lateral side. 


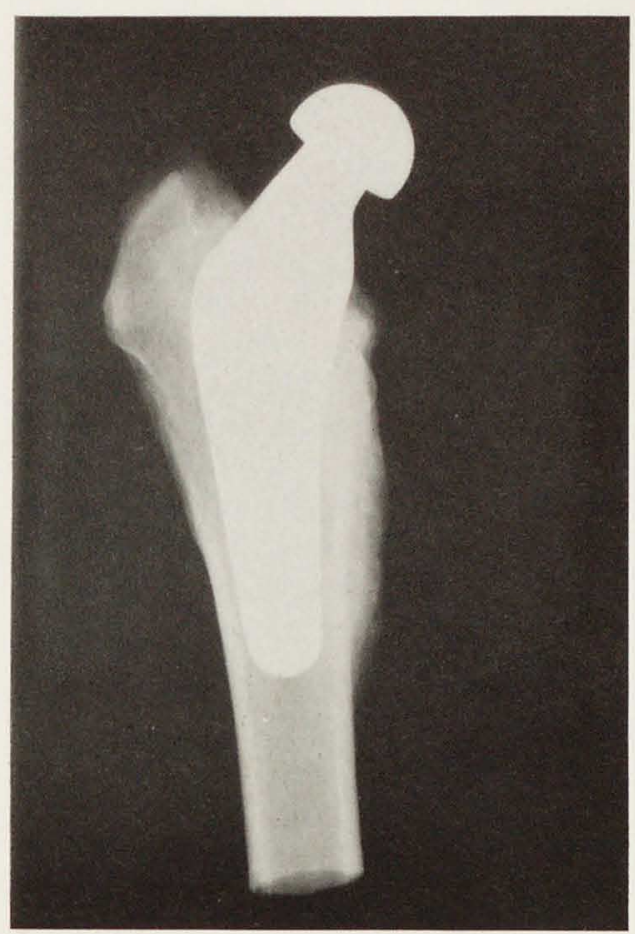

Fig. 86

Periosteal apposition of new bone in response to infection at hydroxyl-apatite coated implant-bone interface at six months. (AP explant radiograph).

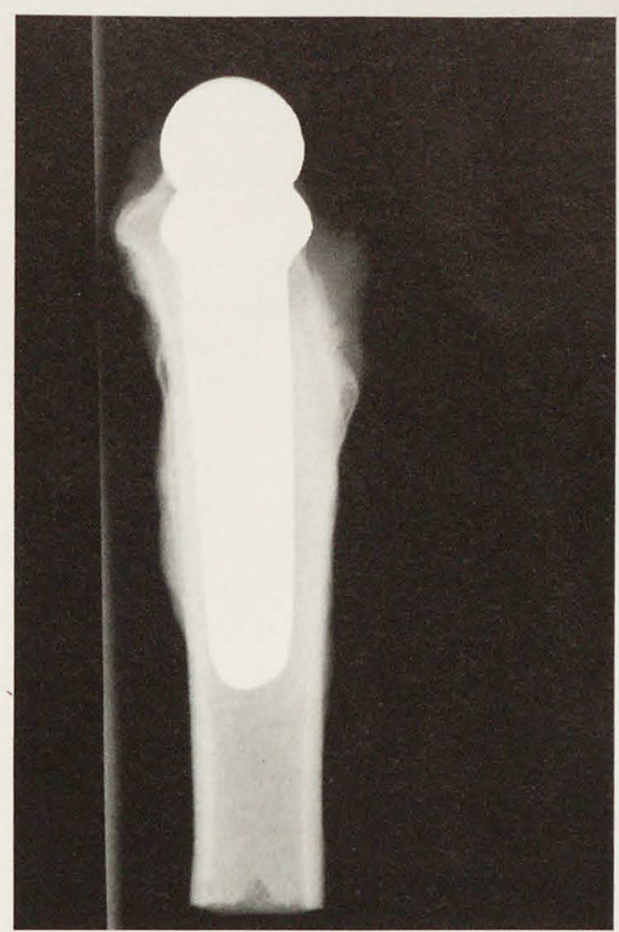

Fig. 87

Same as previous (axial explant radiograph).

\section{Micro-radiographic evaluation}

Micro-radiographic evaluation of the implant-bone interface showed signs of bone resorption in the form of small circular radio-lucencies, comparable to the histological sections.

\section{Histological evaluation}

Microscopic sections reveal that the implant was still fixed to the bone at approximately 25 percent of its surface area.

These areas of bony fixation were scattered over the entire implant surface area. The histological details of these areas with bony fixation are comparable to those detailed in the previous chapter on hydroxyl-apatite coated prostheses. Over the majority of the implant surface area, there were microscopic signs of bone resorption. There were many semicircular shaped areas of focal bone resorption that fused together to a continuous front of bone resorption (Fig. 88). The interface became empty or fibrous on these locations. The thickness of this layer was between $100-200$ microns (Fig. 89) and in this tissue as well in the nearby bone there were many polymorpho-nuclear leucocyte infiltrations. In a few areas local abscess formation was evident in the form of larger localized areas of bone resorption and replacement with fibrous tissue with polymorpho-nuclear leucocyte 


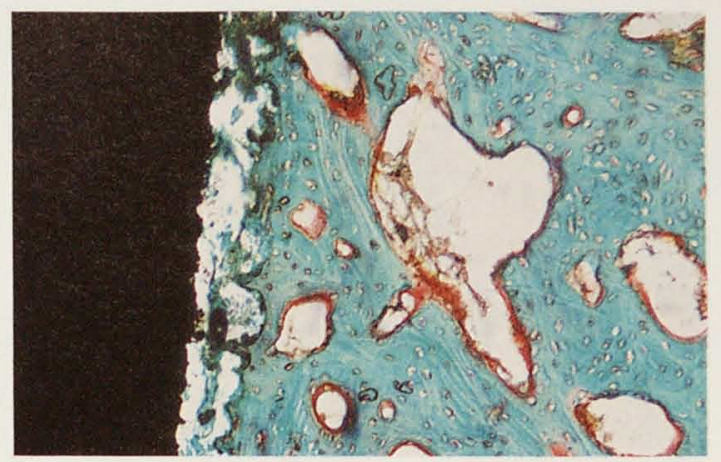

Fig. 88

Bone resorption, micro-scalloping and fragmentation of coating at implant-bone interface of infected hydroxylapatite coated femoral stem (Masson trichrome, six months, $\mathrm{x} 50$ ).

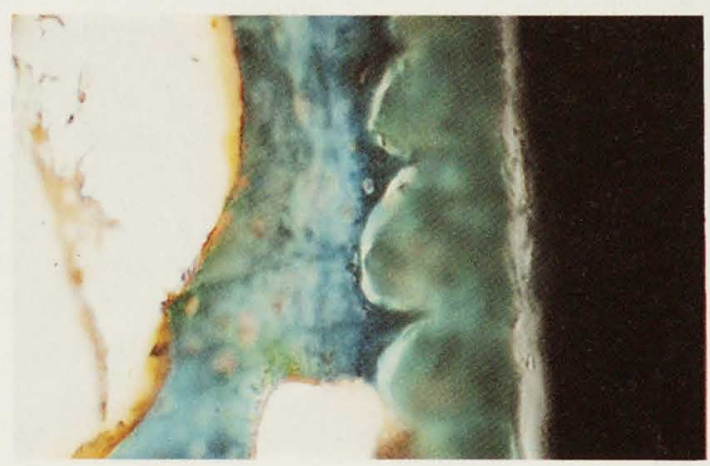

Fig. 89

Bone resorption and fibrous-tissue near infected hydroxylapatite coated femoral stem (Masson-trichrome, six months, x500).

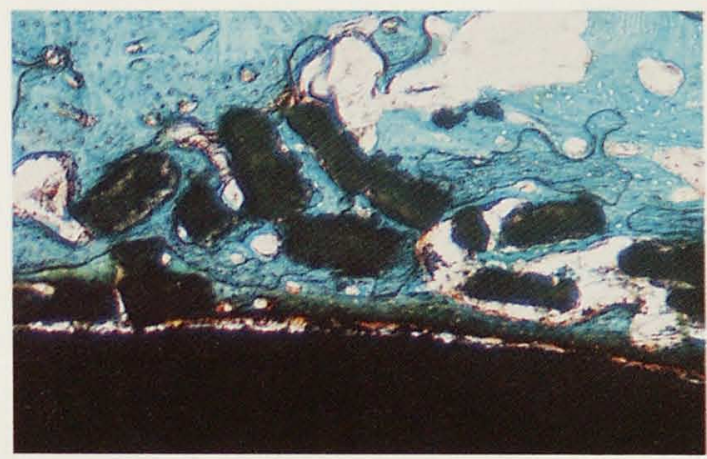

Fig. 90

Severe fragmentation of infected hydroxyl-apatite coating at interface of coated femoral stem (Masson-trichrome, six months, $\mathrm{x} 200$ ). 
concentrations. The quality of the remaining hydroxyl-apatite coating was poor. There was substantial coating fragmentation with loosening and displacement from the substrate metal (Fig. 90). On other places, the hydroxyl-apatite coating was still in place, but discontinuous.

\section{Discussion}

The results with the infected hydroxyl-apatite coated prosthesis indicate, that the implant was initially fixed to the bone in many places. At time of sacrifice, there was still a significant area of implant-bone bonding. Nevertheless, the majority of the implant-bone interface showed signs of active bone resorption with inflammatory response at the implant-bone interface. The thickness of this resorptive layer was minimal, but the result was that the implant became loose from the bone in these locations. Also, the hydroxylapatite coating became fragmented in many places and disappeared in others. At time of sacrifice, this process of bone and coating degradation was still in progress. Thus, it is probable that with some longer follow-up, the whole of the implant-bone interface could have become loose. At the same time, the total amount of bone loss with this infectious complication was not excessive. The thickness of the bone resorption layer was up to 200 microns.

In conclusion, the theoretical consideration that under infectious circumstances, with their lowered tissue $\mathrm{pH}$-levels, the hydroxyl-apatite coating becomes chemically unstable and starts to dissolve has indeed been observed in this animal. These results may not be extra-polated to other causes for infectious conditions with hydroxyl-apatite coatings until more data become available on this topic. Nevertheless, it appears re-assuring from a clinical point of view, that infected hydroxyl-apatite coated implants may eventually loosen. 


\section{Chapter nine}

\section{Human total hip replacement using hydroxyl-apatite coatings}

The concept of chemical bone bonding of hydroxyl-apatite coatings on titanium substrates has proved to be effective in the animal studies performed. The physico-chemical characteristics of hydroxyl-apatite coatings have been determined. The chemical purity and biological stability are acceptable. Static substrate bonding strength is good and there is no susceptibility to fatigue failure of the hydroxyl-apatite coating under repetitive tensile loading up to ten million cycles. The risk of loosening of the coating from the titanium substrate is therefore low. The plug study proves a bone-bonding shear strength of $30 \mathrm{MPa}$. The histological results confirm the close bonding between hydroxyl-apatite coating and bone.

The canine total hip replacement study established the behaviour of hydroxyl-apatite coated implants under conditions of substantial mechanical loading. There proves to be a striking difference in biological response between non-coated and hydroxyl-apatite coated implants, both having the same initial pressfit fixation in bone. Uncoated prostheses are surrounded by a fibrous tissue membrane and are easily extracted from bone.

Hydroxyl-apatite coated prostheses acquire a strong chemical bone-bonding within a time interval of six weeks over their entire surface area. Radiological and bone scintigraphic findings prove a good bony integration of hydroxyl-apatite coated implants. No adverse tissue response has evolved thusfar. Therefore, the transfer of the chemical bone bonding concept to human applications appears safe.

Based on the results of our investigations, there emerge some factors that need special attention in developing human applications with hydroxyl-apatite coated implants. For an optimal mechanical, biological and thereby clinical result, the following details need discussion:

\section{- implant factors}

materials

cross-sectional geometry

implant sizes

area of hydroxyl-apatite coating

- surgical factors

indications for operation

preoperative planning

surgical technique

postoperative regime 


\section{Implant materials}

In our studies, we proved the strong bonding between plasma-sprayed hydroxyl-apatite and titanium. There is no difference in substrate bonding between the frequently used titanium alloys $\mathrm{ASTM}^{3} \mathrm{Ti}-\mathrm{F} 67$ or Ti-F 136. The substrate-bonding strength is high enough to prevent loosening of the hydroxyl-apatite coating from the substrate metal. Although other frequently used metals, like chrome-cobalt alloys, can also be coated, their substrate-bonding strength is unknown. Coating technology has to be established before their use with hydroxyl-apatite coatings is justified. Additionally, titanium is a safe metal for implantation purposes. There are no reports on allergic or carcinogenic effects thusfar.

\section{Prosthesis design}

For optimal bony fixation to occur, the prosthesis must have mechanically stable seating in healthy bone. Absence of gross micro-motion at the implant-bone interface and a well vascularized bony surrounding are important prerequisites for bony fixation.

Both geometric and metallurgic parameters of the implant are responsible for achieving this stable seating without gross micro-motion. Because trabecular bone is not able to withstand high loading forces, the prosthesis must rest on the stronger cortico-spongious or cortical bone. In practice this means that the prosthetic geometry should have a close conformity to the internal anatomy of the proximal femur. With reduced surface contact areas, higher relative stresses at the implant-bone interface are likely to occur, adversely influencing implant-bone bonding by causing more micro-motion.

At the same time, the stiffness of the implant may not be too great. Under normal loading conditions of an implant, the distribution of stresses between implant and bone is mainly determined by the relative stiffness of the implants. The stiffer of the two materials will take the major part of the stress, by preventing deformation of the other component. The stiffness of an implant is mainly determined by its cross-sectional geometry and its modulus of elasticity. Too low a stiffness of a prosthesis (a flexible implant) does not prevent micro-motion under bending forces in a curved structure like a proximal femur. Too high a stiffness of the implant will take the stress away from the bone by preventing bone deformation. This stress-shielding will cause subsequent bone resorption. Although the cross-sectional geometry of the implant is more important than the modulus of elasticity of the metal in preventing bone resorption by stress-shielding, it still is a significant factor. This is a main reason for preference of titanium over chrome-cobalt alloys for use with cementless implants. Its modulus of elasticity $(110.000 \mathrm{MPa})$ is only half that of chrome-cobalt alloys (230.000 MPa), although titanium still is much stiffer than cortical bone. Optimum geometrical design is still a subject of continuing research. Because implant design is heavily affected by the method of implant fixation, further studies must be conducted towards optimalization of implant geometry in relation to hydroxyl-apatite coatings.

3 American Society for Testing and Materials 


\section{Implant sizes}

In practice there is wide variation in size and geometry of the proximal as well as distal femur. To limit the number of available implant sizes to a practical level, it is necessary to apply proper sizing instruments to adapt the femoral bone to the prosthesis. They include intramedullary reamers and calcar rasps, six to seven incremental femoral and acetabular sizes appear the minimum required number. After proper reaming, a slightly oversized prosthesis, in relation to the prepared intra-medullary canal, is "press-fit" into the femoral shaft to provide a tight initial fit. Chemical fixation to bone of the implant by the hydroxylapatite coating will then be obtained within three to six weeks in areas with primary bone contact.

Any remaining slits and spaces will then ossify in the subsequent weeks and months, provided the space is not more than 2 millimeters wide.

\section{Area of hydroxyl-apatite coating}

One question still concerns the area of hydroxyl-apatite coating on the stem of the implant. To promote proximal transfer of stress on the prosthesis, which is the preferred method of stress transfer in an artificial hip joint, a partial-proximal coating of the implant stem could be beneficial. The acquired bone-implant interface-strength is high enough to assure physiological tissue strain levels, even with a reduced surface area of fixation. Too much distal fixation of the implant stem would anyway give rise to proximal stress-shielding effects. Thus, very little is lost in the use of implants with proximal hydroxyl-apatite coatings. Extra benefit is gained in case revisional surgery becomes necessary, for example in cases of malposition or infection. The proximal coating area is still accessible with thin osteotomes and related instruments to separate it from the bone. More distally coated stems would become increasingly difficult to remove. In the animal studies performed, the conclusion was drawn that proximal coatings have preference, because of the lower risk of stress-shield phenomena. Rigid distal fixation of the fully-coated implants was shown to lead to a larger incidence of proximal loss of cortical thickness in the femur. Also from other animal studies (Turner ea. 1986) it is known that complete circumferential coatings (porous-metal coatings this case) induce more bone resorption by stressshielding.

Thus, partial coating of the proximal stem area has certainly preference in primary total hip replacements. A fully coated stem could be reserved for special indications, like revisions with loss of bone-stock. More detailed answers to these questions are contingent on the results of further studies. The technical problems related to the handling of partially coated implants are detailed in the chapter on plasma-spraying technique.

\section{Surgical factors}

\section{Surgical preparation}

Even with good implants, the surgeon in the operating room is responsible for accomplishing a correct fit of the implant in the bone. As discussed earlier, primary mechanical stabilization of the prosthesis in the bone is necessary to prevent early micromotion. Because biological processes are involved in the process of acquiring bony union, vascularization of the implant bed is a second important factor. This dictates, for instance, 
that acetabular reaming should proceed until at least some bleeding is encountered in subchondral bone. On the femoral side, reaming should not go too far. Excessive reaming can cause thermal necrosis of bone, while exposing the prosthesis to low vascularized cortical bone. Reaming should proceed until the transition area of strong corticospongious to cortical bone. A high level of surgical skill is necessary to accomplish "primary close fit" between implant and bone. Good instruments and judicious preoperative planning can prevent many problems. Although the procedure of implanting a hydroxyl-apatite coated prosthesis is certainly more forgiving than an otherwise similar non-coated one, too much motion at the prosthesis-bone interface, through malposition or undersizing of the implant, can adversely influence bony fixation. The exact limits of this tolerance are not yet known.

\section{Postoperative weight-bearing regime}

For optimal bony ingrowth of the implant, some postoperative period of protected weightbearing is recommended. Bony union seems to proceed rather quickly, more quickly than with current cementless implants of bioinert materials. Until more clinical data become available, an interval of four to six weeks of partial weight-bearing seems advisory. From experimental data (Gitelis ea. 1982) the effect of a short postoperative period of protected weight-bearing appears already beneficial for cemented implants to bridge the time for recovery from tissue trauma caused by the surgical trauma of implanting a prosthesis. Similar arguments hold true for cementless implants.

\section{Indications for hydroxyl-apatite coated prostheses}

Indications for hydroxyl-apatite coated implants are active patients with good primary bone-stock, especially in the younger age groups, below 60-65 years of age, where longterm results of cemented total hip replacement might become less satisfactory. Typically, these patients have high bone metabolism, that can respond rapidly to the new situation of a cementless hip with its altered stress pattern in the bone. Reumatoid arthritis or metabolic bone disease like severe osteoporosis, probably represent relative contraindications for hydroxyl-apatite coated implants. Although cemented hip prostheses under the same circumstances also have lower performance, they are probably more sure in realizing short term satisfactory results. Until more clinical experience is gained, this will remain a disputed matter.

This does not mean that biological fixation using hydroxyl-apatite coated prostheses in the higher age groups should in itself be less satisfactory. In a recent literature report (Osborn 1987), the first human autopsy retrieval of an hydroxyl-apatite coated hip prosthesis is discussed. It concerns an 84 year old lady, dying of a pulmonary embolus, seven weeks after operation. The histology reports good bony fixation and bony coverage of the entirely coated femoral stem. Although this is only a single observation, it still proves that biological fixation is not impossible in older patients.

Nevertheless, results of cemented total hip replacement in the older age groups usually are excellent and with our current knowledge and experience, cemented implants represent the most reliable way of mobilizing older patients with a minimum of postoperative discomfort. The lower bone metabolism in elderly patients would probably require a more prolonged postoperative period of restricted weight-bearing. This causes considerably more discomfort with elderly patients as compared to younger ones. There are however 
many patients who fall between these two categories. They may have less than ideal bone quality, but still have a relatively long life expectancy. The ultimate choice then, depends on the individual characteristics of the patient, his life style and general health, but also on the experience of the surgeon with the procedure.

\section{Human implantations of hydroxyl-apatite coated hip prostheses}

First human implantations of hydroxyl-apatite coated hip prostheses were performed by the author in 1986. Although longterm performance of orthopaedic implants constitutes the ultimate test criterion, early clinical performance is still important. It is of crucial importance for any kind of human implant, that adequate functional results be obtained in a reasonably short period of time. The main disadvantage of many current cementless hip implants is their inadequate short term performance with "mid-thigh pain", limping, and related problems. Although this may disappear after one or two years, in the mean time, functional recovery of patients is incomplete and unsatisfactory. From a theoretical point of view, hydroxyl-apatite coated implants should permit a much shortened recovery period, because of their more rapid bony fixation. If the short term clinical results of hydroxyl-apatite coated implants should prove very unsatisfactory and fraught with many problems and unsuspected traps, then the whole concept would become unsettled. For this reason, some early clinical impressions of results are presented in the following paragraphs. The real longterm follow-up results are beyond the scope of this investigation and are the subject of future work.

\section{Patient statistics}

Data pertaining to clinical results can be divided in two categories. The first are data on functional recovery, as reflected in the Harris hip ratings. The most significant changes in clinical performance are to be expected in the first three to six months after operation. Longterm follow-up is necessary in order to evaluate how well these clinical results are preserved in the long run. The second category of data relates to the objective analysis of the implant-bone interface. Radiographic and bone scintigraphic evaluation of the implant-bone interface are important in this respect. They become more important with longer follow-up. The first four to six months after operation reactive changes in the bone, caused by remodelling, are hardly visible on radiographs.

Additionally, bone scintigraphic findings of the bone-bonding process early after operation are difficult to evaluate, because of the high bone uptakes caused by the surgical trauma. These radiographic and scintigraphic data do not always directly correlate with clinical performance. Nevertheless, they give valuable information on the interface characteristics of the implant and the preservation of bone stock. They are a very important prognostic factor to the life expectancy of the individual implant (Tapadiya ea. 1984, Ilstrup ea. 1973). We will focus on the early data given by the Harris hip ratings about the speed of functional recovery after operation. They are the most relevant signs for patient satisfaction with the procedure. Furthermore, the normal evolution of the radiographic appearance of the hydroxyl-apatite coated implant-bone interface will be detailed as well as early findings with isotope bone-scanning (technetium and indiumlabeled leukocytes). 


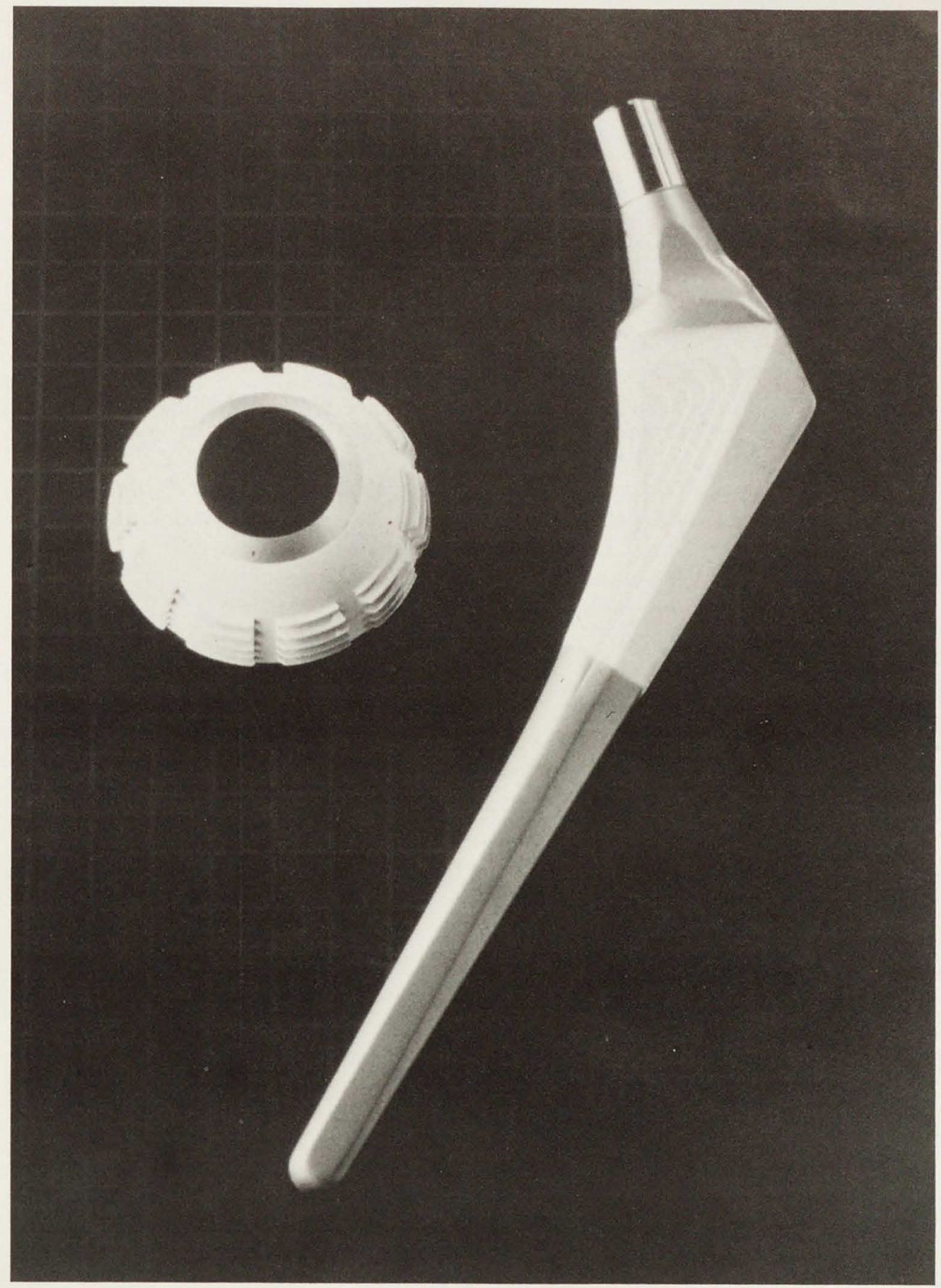

Fig. 91

Omnifit ${ }^{\circledR}$ hydroxyl-apatite coated total hip prosthesis. 


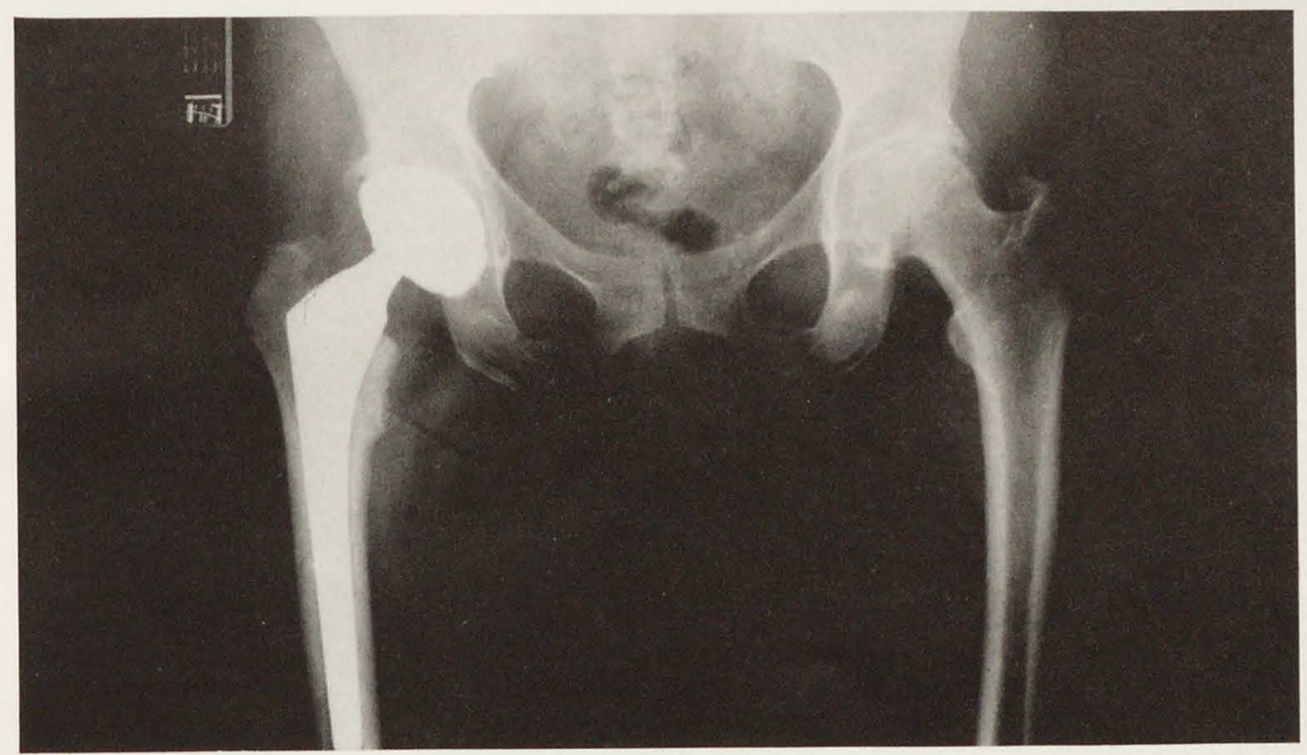

Fig. 92

Human hydroxyl-apatite coated total hip replacement at six weeks (AP radiograph).

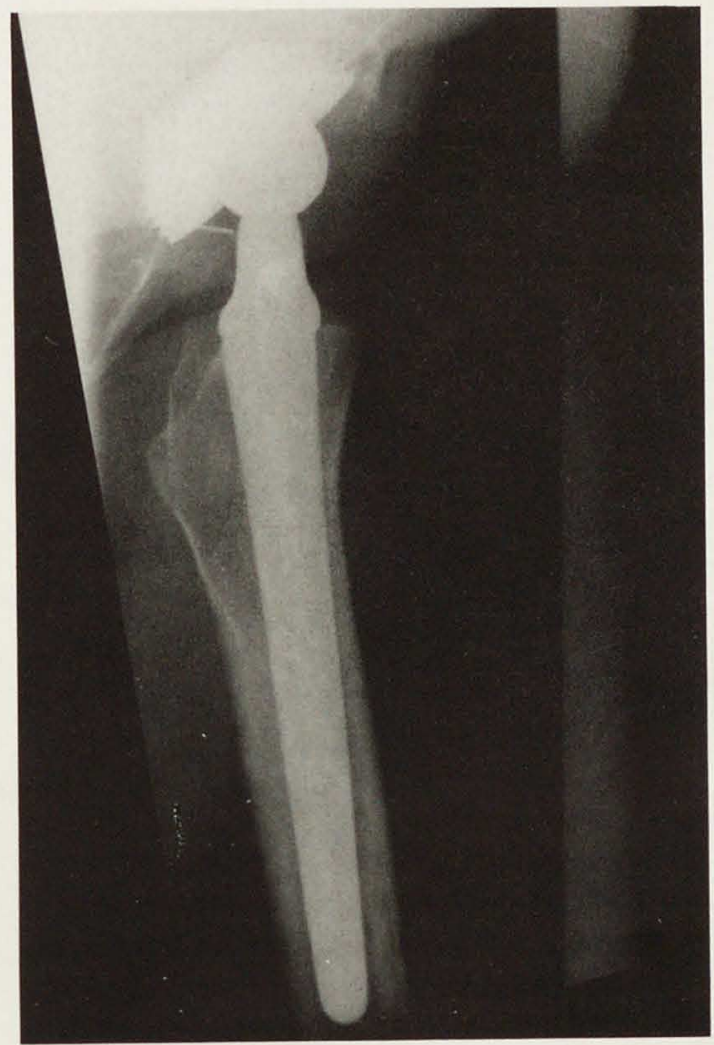

Fig. 93

Hydroxyl-apatite coated total hip replacement at six weeks (axial radiograph). 


\title{
Materials and methods
}

To introduce the results of this study into practice, total hip prostheses of the Omnifit ${ }^{\circledR}$ system (Osteonics $\left.{ }^{(\mathbb{B}}\right)$ were adapted according to the previously described requirements for hydroxyl-apatite coatings. The result is a human total hip replacement system with hydroxyl-apatite coating for chemical fixation to bone, in clinical use since 1986 (Fig. 91, 92 and 93). The following paragraphs will detail the relevant techniques:

\author{
implant characteristics \\ prosthesis \\ instrumentation \\ surgical technique \\ patient selection \\ preoperative planning \\ surgical technique \\ follow-up protocol \\ postoperative regime \\ grading of results \\ patient statistics \\ clinical results \\ radiologic findings \\ bone scintigraphic findings
}

\section{Implant characteristics}

The Omnifit ${ }^{\circledR}$ hip system with hydroxyl-apatite coating has the following characteristics:

- titanium stems in seven sizes

- proximal coating of femoral stem

- acetabular screw-rings in nine sizes

- exchangeable femoral heads

- non-coated implants for cemented applications

- universal instrumentation

\section{Instrumentation}

The specific instruments for preparation of the implant include conical intra-medullary reamers for the axi-symmetric part of the implant and calcar rasps for preparation of the triangular asymmetric calcar component of the implant. Each implant has its own specific reamer and rasp for optimal accuracy.

Patient selection in the study

Patients with a diagnosis of osteo-arthrosis of the hip or an avascular necrosis of the femoral head are included in the series. These are generally patients in good general health, the hip disease being their only significant problem (Charnley's categories A or B). In these categories of patients, the functional results of the operation can be evaluated with the least distortion by otther factors. Charnley's category A patients are healthy people with unilateral hip disease, category B have bilateral hip disease, but are otherwise healthy. Category $\mathrm{C}$ patients have uni- or bilateral hip disease, but compromized with other disease 
affecting their hip performance. By the end of 1987 , there were 50 implants available for evaluation from the author and another 30 from affiliated clinics. The average age was 54 years, with a minimum of 22 and a maximum of 72 years. Male to female ratio was almost $1: 3$. There were 11 operations for avascular necrosis of the femoral head and the remainder mainly for osteo-arthrosis of the hip. Patients with revisions of prosthetic components (six), fractures (two), inflammatory disorders or related collagen diseases (two) represent different groups, that will be detailed separately.

\section{Preoperative planning}

It is generally believed that preoperative planning adds to the accuracy of the operative procedure (Capello 1986). With the aid of specific templates, the probable implant size can be determined and the optimal resection level of the calcar selected for best fit of the implant in the bone and preservation of correct leg length.

\section{Surgical technique}

After adequate surgical exposure of the diseased hip joint, the femoral head is luxated and its neck resected at the predetermined level with the aid of specific instruments. Intramedullary reaming is then performed with incremental sizes of reamers until adequate surgical feel of proper reaming. The corresponding calcar rasp then prepares the calcar area before insertion of a slightly undersized trial implant. Femoral preparation is slightly undersized $(0.7 \mathrm{~mm}$.) as compared to the final implant so as to allow a mechanically "tight fit" between implant and bone. Acetabular preparation consists of the usual spherical reamers in incremental sizes until spherical congruency with the edges of the acetabulum. Then, a conical reamer prepares the edge of acetabulum for better initial grip of the selftapping screw-thread of the acetabular component in the bone. After adequate trial reduction and control of position of the implants, the final implants are selected and inserted in the femoral canal and acetabulum. Because of the high surface-microroughness of hydroxyl-apatite coated implants, there is more frictional resistance with insertion of these implants as compared to smooth metal implants. Further surgical technique is similar to conventional hip replacement surgery.

\section{Postoperative regime}

Postoperatively, patients are to be exercised and mobilized as soon as general condition and wound healing permit. For optimal bony integration of the implant, patients are mobilized on a restricted weight-bearing regime for the first six weeks after operation. They are allowed $20-25 \mathrm{~kg}$ force load-bearing on the affected limb using two crutches. After six weeks, the crutches are discardéd and patients are further mobilized and exercised until full weight-bearing and functional recovery.

\section{Grading of clinical results}

All clinical, radiographic and laboratory data are collected in a multicenter prospective clinical follow-up study. There are many rating systems available for evaluating hip surgery. The Harris hipscore, the modified Harris hipscore, the Mayo, the Iowa, the Hospital for Special Surgery rating system or the Merle d'Aubigné hipscores all aim at the most objective evaluation of post-operative hip performance. Some systems only use clinical parameters, others also include radiographic parameters. When compared (Edit J Bone Joint Surg 1985), most rating systems prove equally well capable of separating good 
Table VI

Determination of modified Harris hip score

\begin{tabular}{lc}
\hline Category & score points \\
\hline Pain & 45 \\
$\quad$ no pain & 40 \\
slight or occasional pain & 20 \\
$\quad$ moderate pain, causes limitations & 10 \\
$\quad$ marked pain, serious limitations & 0 \\
$\quad$ totally disabled & \\
\hline Limp & 11 \\
$\quad$ no limp & 8 \\
$\quad$ slight limp & 3 \\
$\quad$ severe limp & 0 \\
$\quad$ unable to walk &
\end{tabular}

\section{Support}

$\begin{array}{lr}\text { no external support } & 11 \\ \text { cane with long walks } & 8 \\ \text { cane, full-time } & 6 \\ \text { crutch } & 4 \\ \text { two canes } & 2 \\ \text { two crutches or unable to walk } & 0\end{array}$

\section{Distance walked}

one hour or more

half hour

fifteen minutes

around house or indoors only

11

8

6

4

2

0

bed or wheel-chair

\section{Stair climbing}

normally

able with bannister

4

able some method

2

unable

\section{Sitting}

any chair, one hour

high chair, half hour

4

unable to sit half hour on any chair

\section{Putting on socks or shoes}

with ease

with difficulty

unable to put on shoes or socks 


Category score points

Car driving or public transportation

able

unable

0

Absence of deformity

without functional disturbance

2

with functional disturbance

0

Calculation of range of movement scores

$\begin{array}{llr}\text { flexion } & \left(0^{\circ}-45^{\circ} \times 1\right)+\left(45^{\circ}-90^{\circ} \times 0.6\right)+\left(90^{\circ}-100^{\circ} \times 0.3\right) & \max 78 \\ \text { abduction } & \left(0^{\circ}-15^{\circ} \times 0.8\right)+\left(15^{\circ}-30^{\circ} \times 0.2\right) & \max 15 \\ \text { adduction } & \left(0^{\circ}-15^{\circ} \times 0.2\right) & \max 3 \\ \text { ext rotation } & \left(0^{\circ}-15^{\circ} \times 0.2\right) & \max 3 \\ \text { int rotation } & \left(0^{\circ}-10^{\circ} \times 0.1\right) & \max 1 \\ & & \max \operatorname{sum~} 100\end{array}$

Maximum hip score points for function is max sum times 0.08

This provides 8 points maximum for range of movements

Categories of maximum ratings

Absence of pain

Activities of daily living

23

distance walked $\quad 10$

stair climbing $\quad 4$

sitting

putting on shoes

public transport

$\begin{array}{r}4 \\ 4 \\ 1 \\ \hline 23\end{array}$

Muscular force

22

absence of limp

11

method of support

$\frac{11}{22}+$

Range of movement

absence deformity

range of movement

2
$\frac{8}{10}+$

10

These calculations provide the maximum possible hip score of one hundred for a completely painless normally functioning hip. 
or excellent clinical results from moderate or poor clinical results. Because radiographic parameters and findings for this new type of hydroxyl-apatite coated hip implant are not yet defined, it is more appropriate to choose a rating system, based on clinical results. This seems the more important because in the clinical use of non-cemented hip implants, pain is the most obvious complaint. Although pain is a subjective phenomenon, it still should be an important criterion for evaluating hip surgery. For these reasons, clinical performance of patients in our group with hydroxyl-apatite coated prostheses is rated according to the standard modified Harris hip score (Ilstrup ea. 1973), which is the most widely accepted hip rating system. The individual scoring items are listed in table VI. This hip score rating system gives 100 points for a hip joint with normal performance, which means complete absence of pain, unrestricted daily activities, good muscular force with absence of limping and unrestricted range of movement of the hip. The other end of the scale is zero points for a completely crippled individual. Within this Harris modified rating system, four categories can be discerned, namely absence of pain (max 45 points), activities of daily life ( $\max 23$ points) muscular force ( $\max 22$ points) and the objective hip ranges of movement ( $\max 10$ points). The data collection forms (appendix A) also incorporate items from some other rating systems, so as to allow the (future) Hip Society, Mayo, Iowa or Merle d'Aubigné hipscores to be retrieved from the computer, if necessary, for proper comparison with available literature. For future reference, there is a clear need for a standardized system for evaluating results of total hip surgery (Editorial JBJS 1985). Recommendations for such a uniform system are currently in study by the Hip Society.

\section{Clinical and radiological follow-up}

All categories of patients are followed in a prospective multi-center clinical study. The data collection forms are in appendix A. Follow-up results are obtained specifically preoperatively and with three, six and twelve months after surgery. Thereafter the patient will be seen each year.

With each such a visit a calibrated X-ray of the pelvis is obtained with AP and axial views of the hip. X-ray data are collected both in an analog and a digitised fashion for future comparison.

\section{Blood chemistry}

Routine blood chemistry is monitored for BSE, leucocyte count and differentiation as well as for parameters of liver and kidney function, including aminotransferase, alkaline phosphatase and creatinine. Investigations were done twice a week until discharge from the hospital, usually the third week. If still abnormal, blood chemistry was repeated thereafter until normal values were obtained.

\section{Bone scintigraphy}

99m-Technetium bone scans were obtained with almost every patient at least once, but regularly twice during their follow-up in the first year. $99 \mathrm{~m}$-Technetium bone scanning was performed during the three phases of injection, venous pooling and late bone accumulation respectively. $111 \mathrm{~m}$-Indium labeled leucocyte scans were performed approximately two weeks after surgery on some early cases, but its routine use was discarded after persistent negative results. 


\section{Results}

\section{General comments}

Clinical follow-up is available since 1986. Results are shown for the first 50 patients operated until the end of 1987. At the time of printing of this manuscript (april 1988), 30 patients have a follow-up between six and twelve months and 12 between twelve and eighteen months. There were two bilateral hip replacements. Surgical complications were comparable to other hip replacement surgery. There were no infections, one postoperative luxation of a hip and one early postoperative fissure of the hip, that healed spontaneously without further effects on clinical performance. All problem cases will be detailed at the end of the description of the normal postoperative course.

\section{Clinical results}

The mean Harris hip ratings pre- and postoperatively, are summarized in table VII. Graphic representations of clinical results and regain of hip range of movement are pictured in Fig. 94 and 95.

Table VII

Functional results after hydroxyl-apatite coated total hip arthroplasty:

Harris hipscores and categories

Months of follow-up

\begin{tabular}{llllllll}
\hline Category & preop & $-1-3$ & $3-6$ & $6-9$ & $9-12$ & $>12$ \\
\hline Hip score & 29 & - & 76 & 88 & 97 & 96 & 98 \\
Absence pain & 9 & - & 41 & 43 & 45 & 44 & 45 \\
Activities DL & 8 & - & 16 & 19 & 22 & 23 & 22 \\
Muscle force & 7 & - & 11 & 17 & 21 & 19 & 21 \\
Range movement & 5 & - & 8 & 9 & 9 & 10 & 10 \\
\hline
\end{tabular}

The average preoperative Harris hip score was 29 , indicating a high level of pain with serious restrictions in activities of daily life and hip functions. The average Harris hip score after six months was 97 , indicating complete absence of pain with excellent recovery of daily activities and only slight remaining impairment in muscular force or range of movement of the hip. Absence of pain is almost immediate after surgery. Recovery of activities of daily life, muscular force, and restoration of hip range of movement take more time. They show a more linear increase with time after surgery, continuing even after the sixth month.

There were few early pain problems. There were two patients sustaining some ischiadic type of pain in the whole leg for up to three or four months postoperatively. There was no relation of this pain with joint-loading or otherwise using the hip. Both complaints resolved spontaneously within four months after operation. Another patient had lateral- 


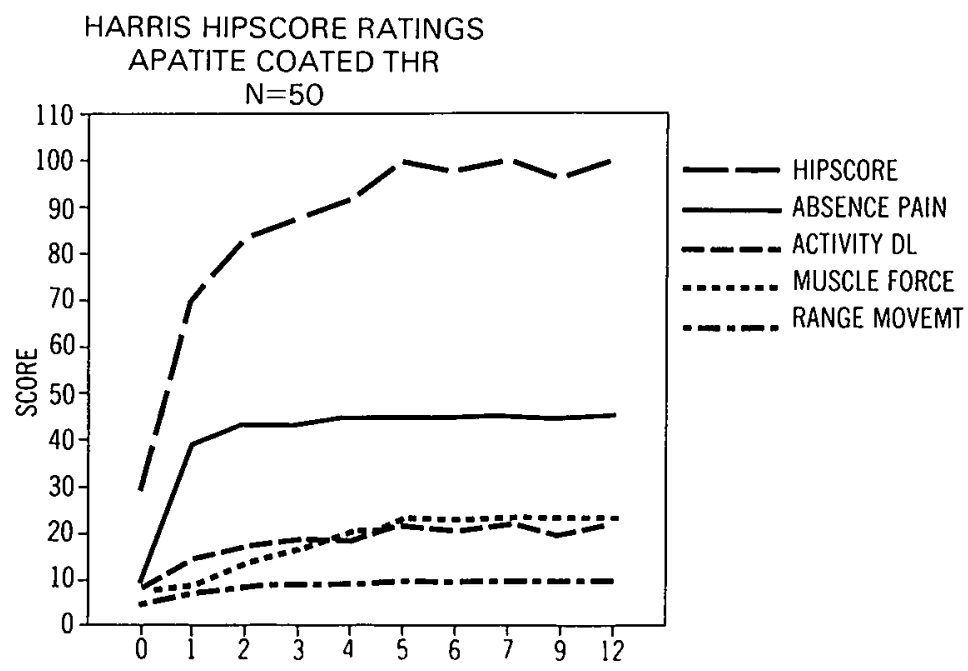

Fig. 94

Graphical representation of Harris hipscore ratings, together with separate pain, ADL, muscular force and ROM ratings of hydroxyl-apatite coated total hip replacements.

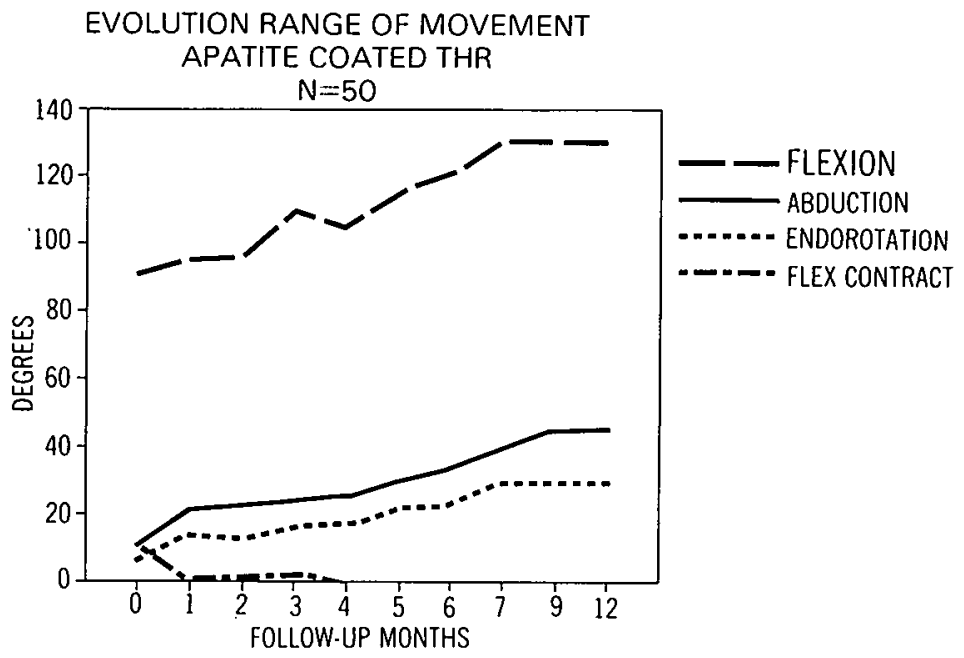

Fig. 95

Graphical representation of restoration of hip range of movements after hydroxylapatite coated total hip replacement. 
thigh pain early after surgery, disappearing in the fourth month after surgery.

There were two patients in category $\mathrm{C}$ with unsatisfactory results, both caused by periarticular calcifications. Although both patients were painfree at follow-up, they exhibited marked impairment of range of motion of the hip. They will be discussed later on. Of the group of fifty patients $35 / 50$ had Harris hip score ratings of 95 or higher, thus representing excellent early clinical results. Furthermore, there appears no difference in clinical results between primary implantations and revisional cases.

\section{Blood chemistry}

Monitoring of blood chemistry after hydroxyl-apatite coated hip surgery did not reveal pathologic changes. The average rise of BSE was up to $70 \mathrm{~mm}$, although five patients had an increase up to $110 \mathrm{~mm}$. BSE. Three weeks after surgery, many BSE ratings were descending and had almost returned to their preoperative levels. There were no persistent elevations of BSE without preexistent disease. Estimations of transaminase, alkaline phosphatase and creatinine were all within the accepted ranges for normal values at all times post-operatively.

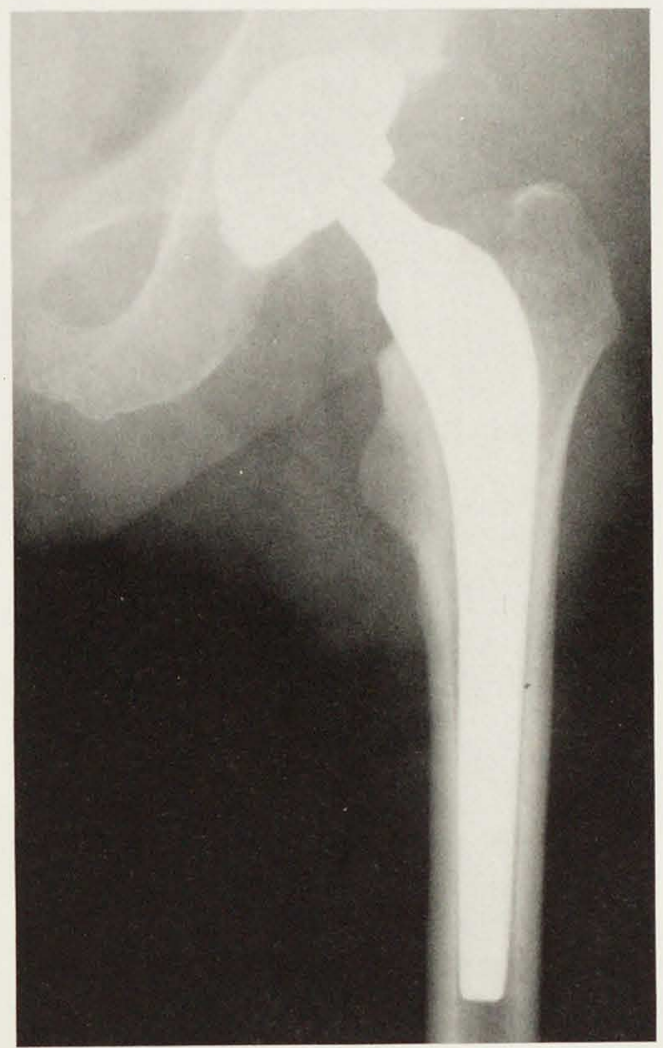

Fig. 96

Densification of bone around hydroxyl-apatite coated part of prosthesis in proximal femur at six months.

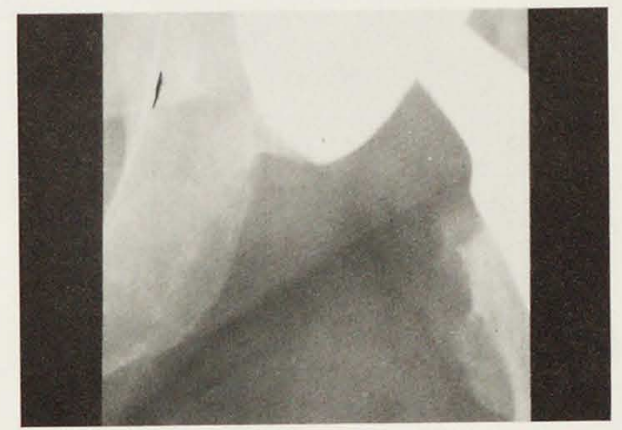

Fig. 97

Signs of new bone formation at lower end of acetabular component and resected calcar edge caused by exposed parts of hydroxyl-apatite coating at three months. 


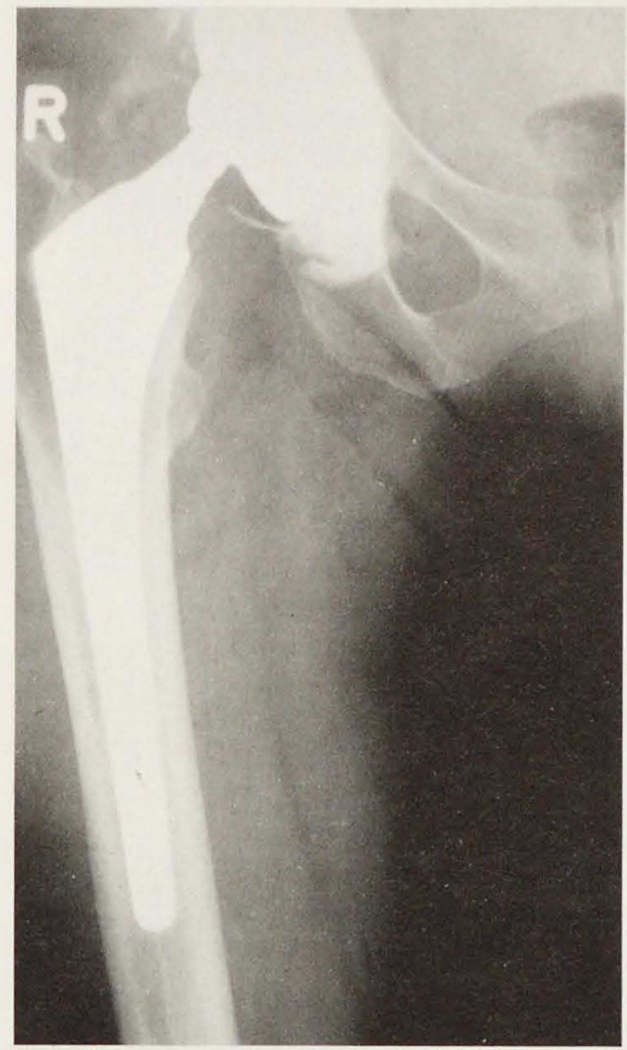

Fig. 98

Densification of bone around hydroxyl-apatite coated part of prosthesis in proximal femur, especially near transition to non-coated part of implant in mid-stem. Line formation around distal non-coated part of component at one year. Revision of double cup prosthesis with use of cemented acetabular component.

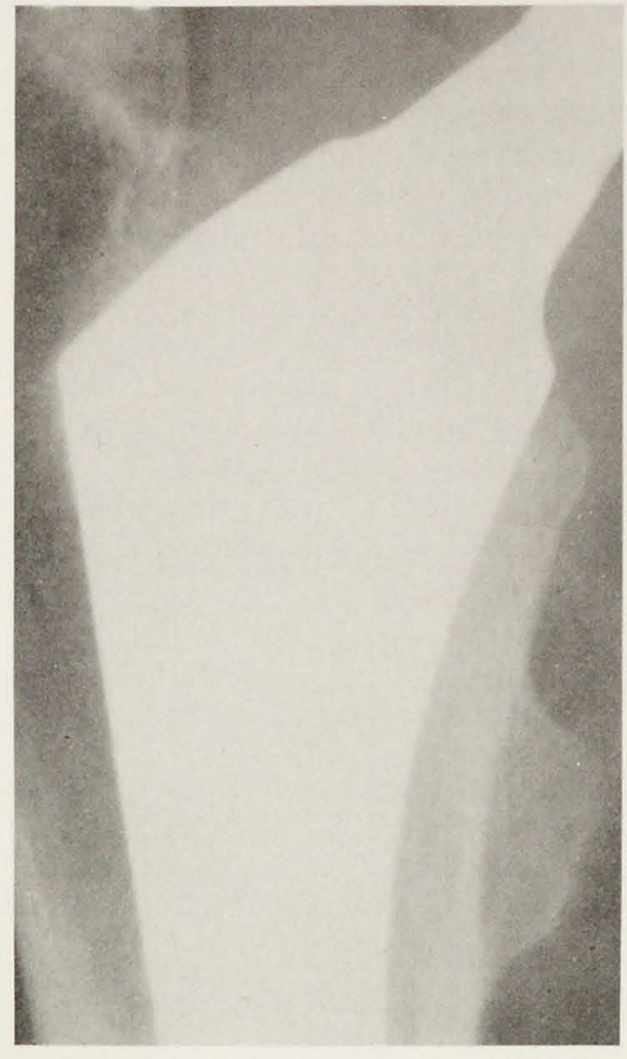

Fig. 99

Same as previous, detail of proximal femoral stem. Absence of radio-lucent line formation and densification of bone around hydroxyl-apatite coated part of prosthesis in proximal femur.

\section{Radiological findings}

In the first three to four months postoperatively, hardly any changes in bony structure are evident. The trabecular structure of the bone is well preserved and there is complete absence of any radio-lucent line formation or any other specific interface line development (Fig. 96). Additionally, there are sometimes subtle signs of new bone formation against bare exposed parts of the coating, for instance, a free area near the resected calcar femoris or the uncovered lower part of the acetabular screw-ring (Fig. 97).

From the fourth to sixth month on, there is a gradual increase in density of the bone in contact with the hydroxyl-apatite coating (Fig. 98, 99, 100 and 101). This increase in bone density occurs over the entire proximal femur, both at its medial and lateral aspects, but is accentuated near the termination of the hydroxyl-apatite coating in the mid-part of the 
femoral prosthesis. Around the distal non-coated part of the femoral component, there regularly (approximately $40 \%$ of cases) develops a very thin radio-lucent line, which is most marked near the stem-tip. There is no accompanying console formation, which is bone formation distal to the stem tip.

Bone density around this distal part of the femoral prosthesis is generally lower as compared to the proximal femur (Fig. 102). With time, especially near the one-year interval, the process of condensation of endosteal bone around the hydroxyl-apatite coated part of the implant becomes more pronounced (Fig. 103, 104 and 105). Radio-lucent lines around coated parts of implants remain completely absent up to longest follow-up with both femoral and acetabular components. At the acetabular side of the prosthesis, changes in bony structure are less apparent. Nevertheless, bone quality remains good and there is complete absence of radio-lucent line formation. Revisional cases showed in general the same radiological processes as those detailed with the primary implantations. Small areas with deficient bone contact filled up and the cortical wall thickness increased to some extent (Fig. 106 and 107).

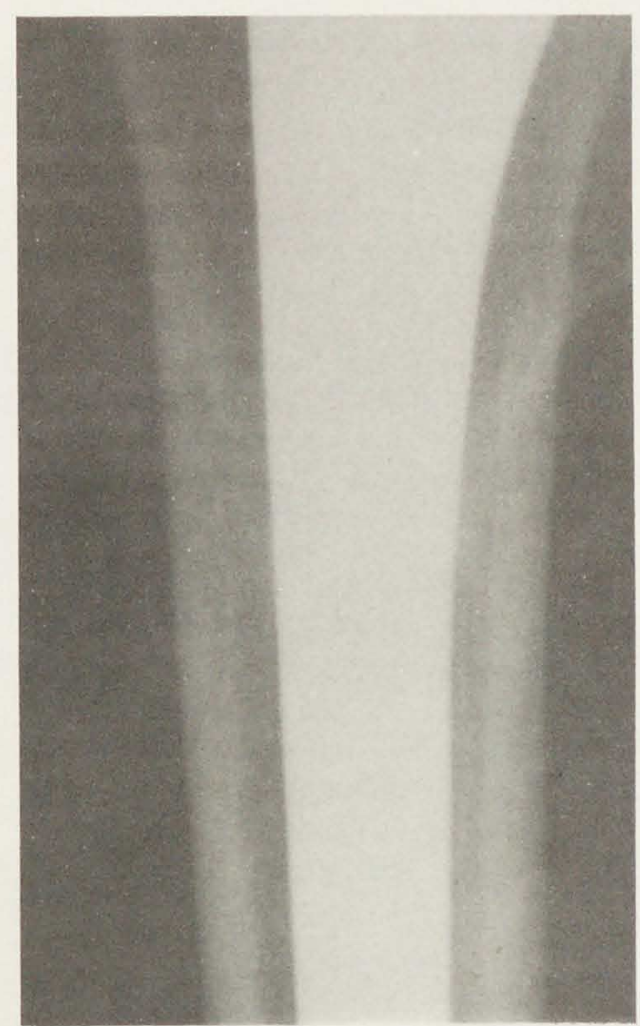

Fig. 100

Same as previous, detail of mid-stem. Densification of bone around hydroxyl-apatite coated part of prosthesis in proximal femur, especially near transition to non-coated part of implant in mid-stem.

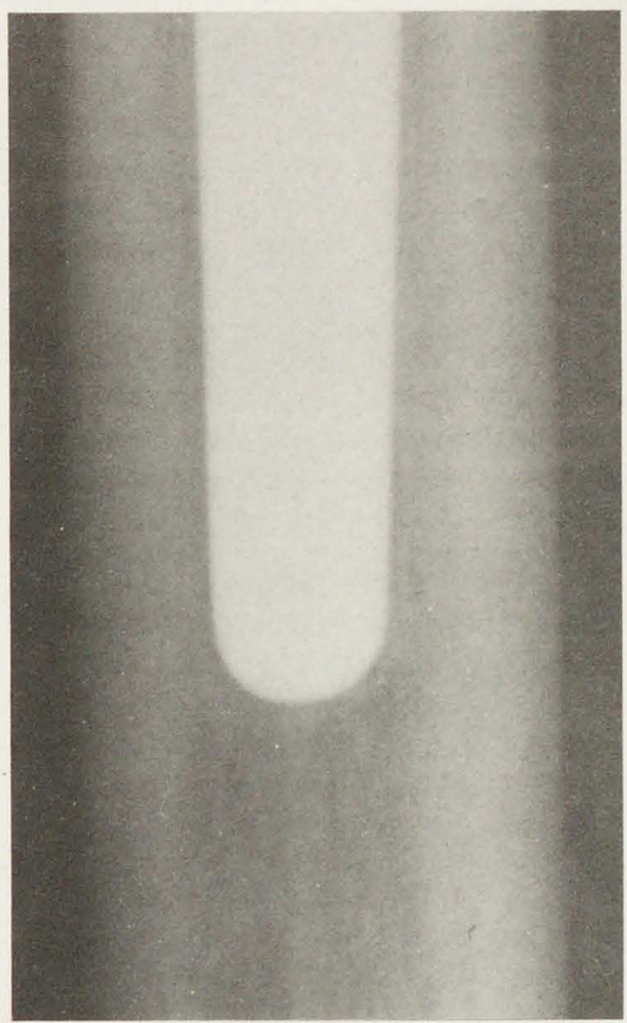

Fig. 101

Same as previous, detail of distal femoral stem. Thin line formation around non-coated stem tip of prosthesis. 


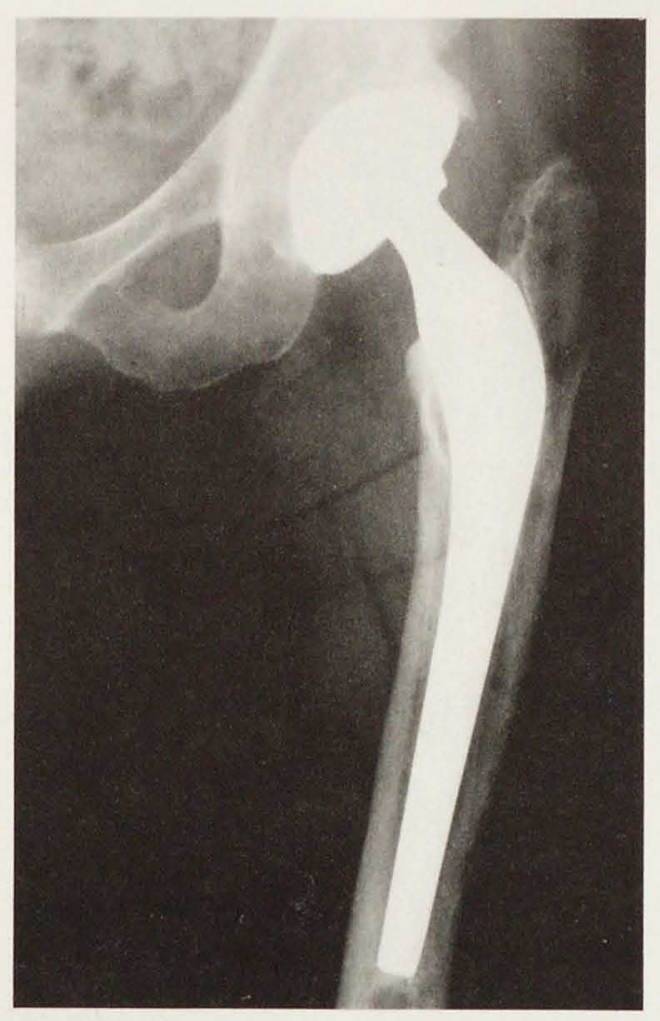

Fig. 102

Hydroxyl-apatite coated total hip replacement at one year follow-up. Difference in bone density around proximal and distal femoral stem.

\section{Technetium and indium scanning}

The specific scintigraphic patterns for hydroxyl-apatite coated implants are only manifest during the late bone accumulation phase of bone scintigraphy. The injection and venous pooling phases shortly after injection of the radio-nuclide do not provide specific information different from other hip surgery. $99 \mathrm{~m}$-Technetium bone scans early after operation (within three weeks) show a high uptake of technetium over the entire proximal femoral stem area. This uptake is generally somewhat higher over the non-coated distal part of the femoral prosthesis as compared to the coated part of the implant in contact with the proximal femur (Fig. 108). Beginning two to three weeks after surgery, technetium uptake gradually diminishes until with six months a low rate of uptake is obtained with an even distribution of radio-nuclide activity over the entire area of hydroxyl-apatite coating. The technetium uptake is somewhat higher over the proximal hydroxyl-apatite coated part of the prosthesis, although uptake remains at a higher level as compared to the nonoperative side, especially near the distal termination of the coating. (Fig. 109, 110 and 111). With six months and longer, uptake is very low and remains localized over the proximal femur, in contact with the hydroxyl-apatite coated part of the implant. Hot spots or other areas of focal accumulation of radio-nuclides were generally absent. Nevertheless, in approximately forty percent of cases there is persistent marginally elevated uptake of technetium around the stem-tip of the femoral component (Fig. 112 and 113). 


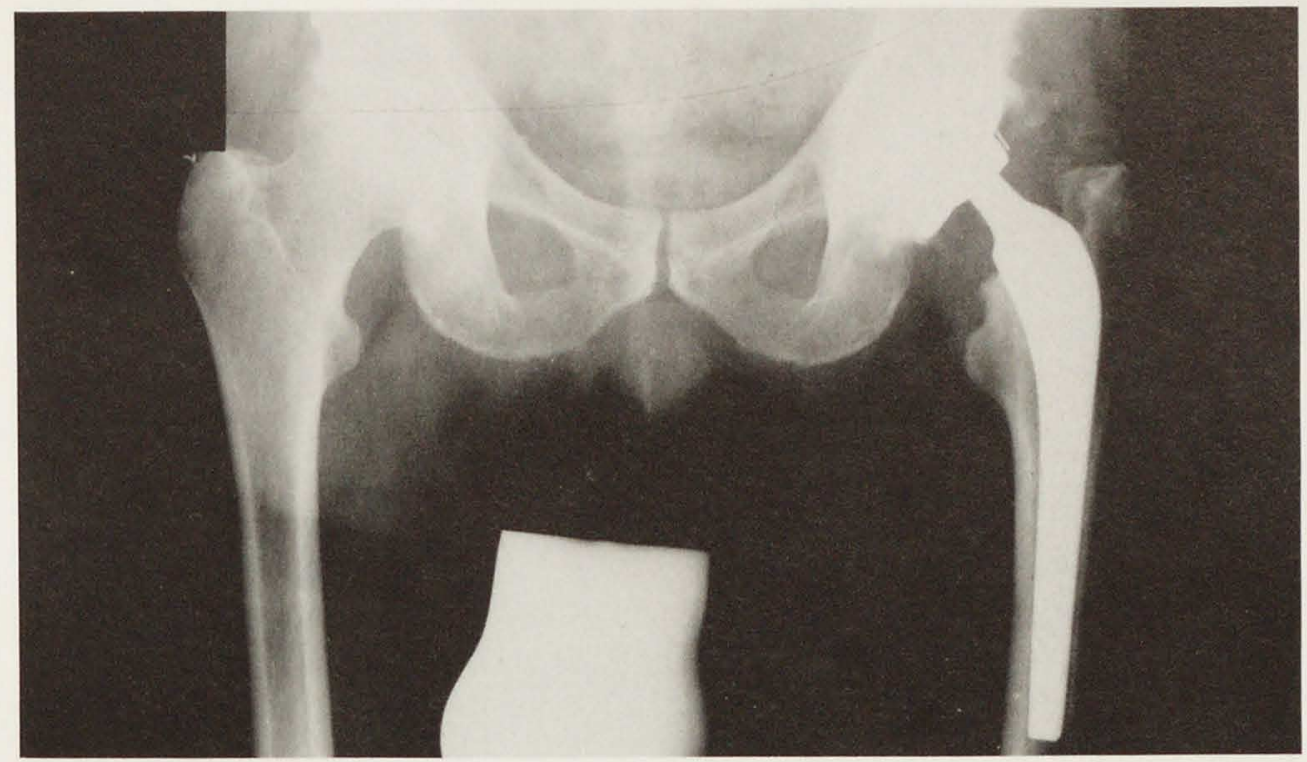

Fig. 103

Hydroxyl-apatite coated total hip replacement, early post-operative radiograph.

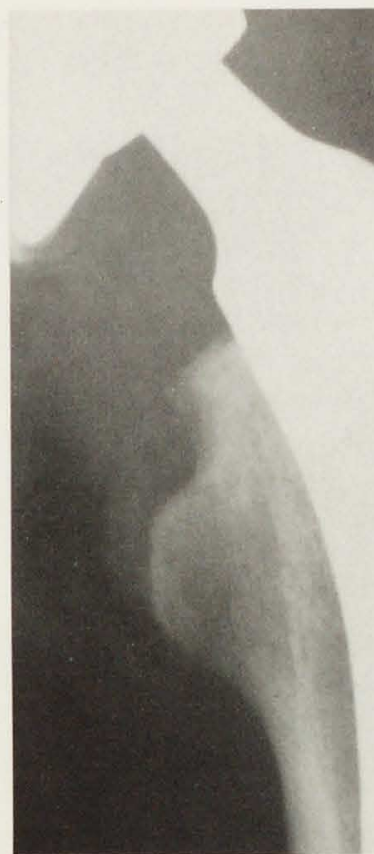

Fig. 104

Same case as previous, detail of femoral stem three months after surgery.

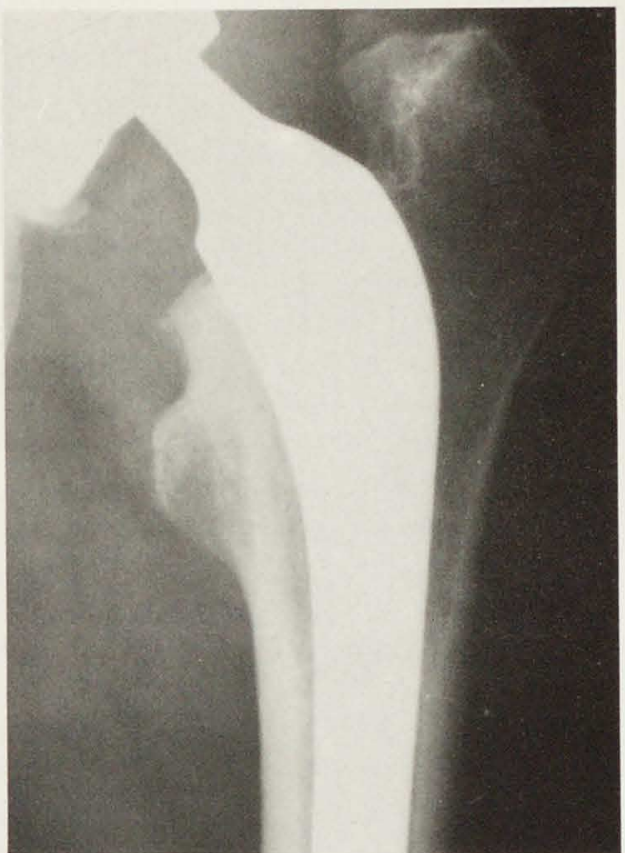

Fig. 105

Same case as previous, detail of femoral stem one year after surgery with increase in bone density in proximal femur. 


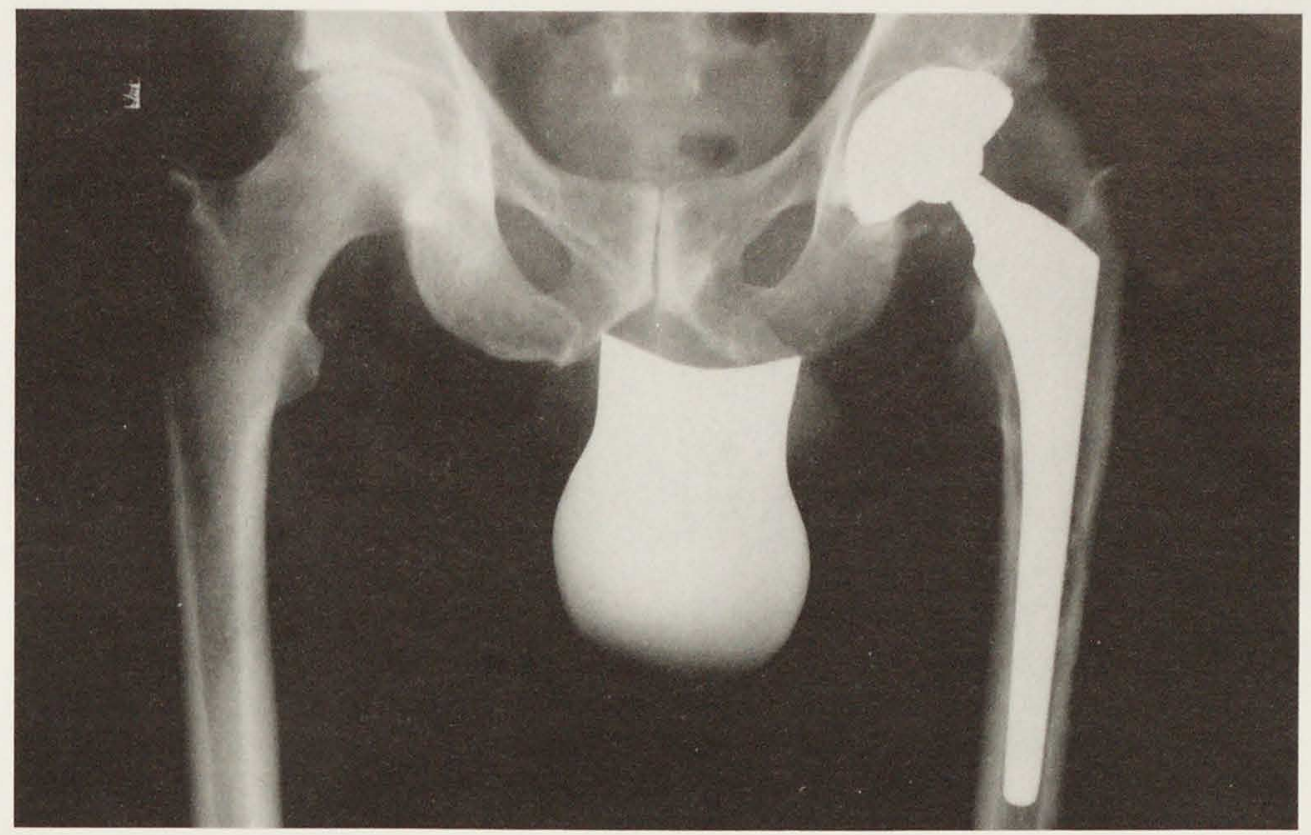

Fig. 106

Revisional case. Replacement of loose cemented total hip replacement by hydroxyl-apatite coated implant, early post-operative radiograph.

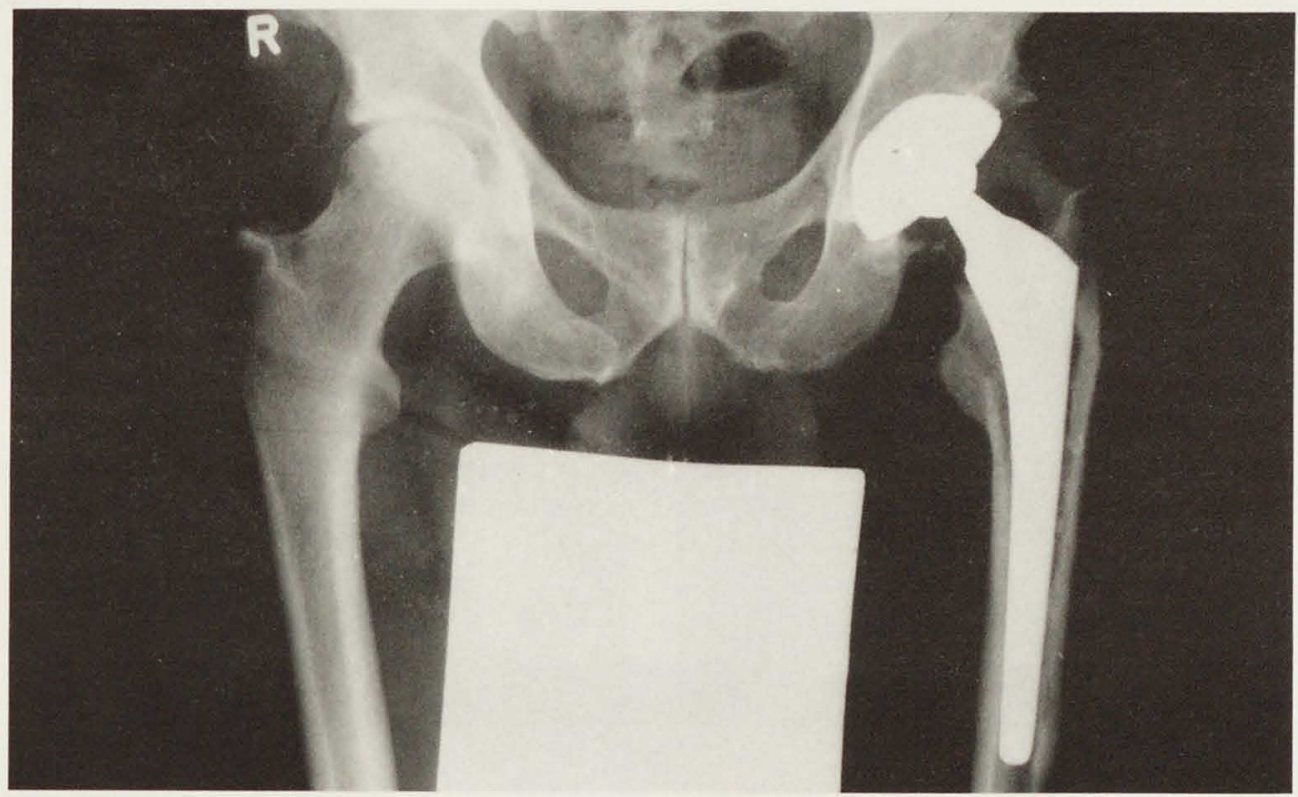

Fig. 107

Same case as previous three months after surgery. 

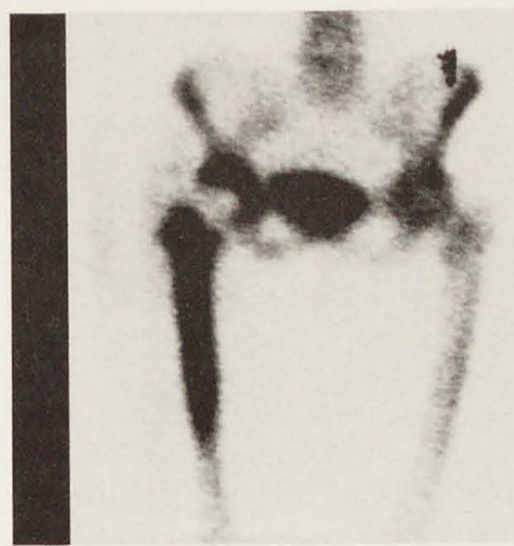

Fig. 108

Technetium bone-scan of human hydroxyl-apatite coated total hip replacement, two weeks after surgery. High uptake over entire femoral stem and acetabular area.
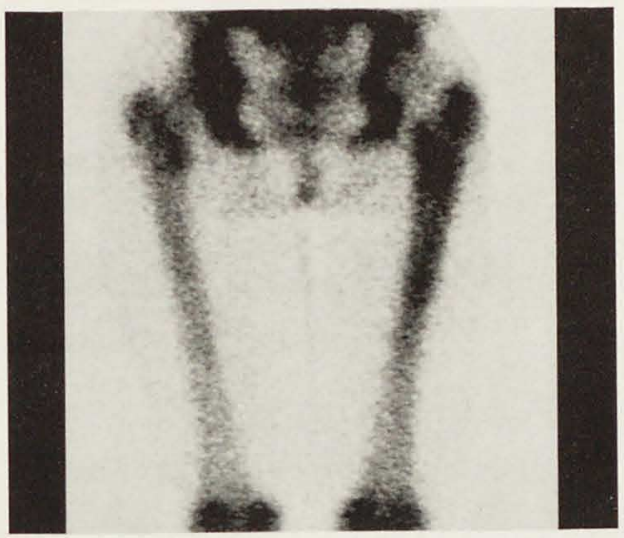

Fig. 110

Technetium bone-scan of human hydroxyl-apatite coated total hip replacement, three months after surgery. Activity still present in entire proximal femoral stem area, although at a lower rate as compared to earlier postoperatively.
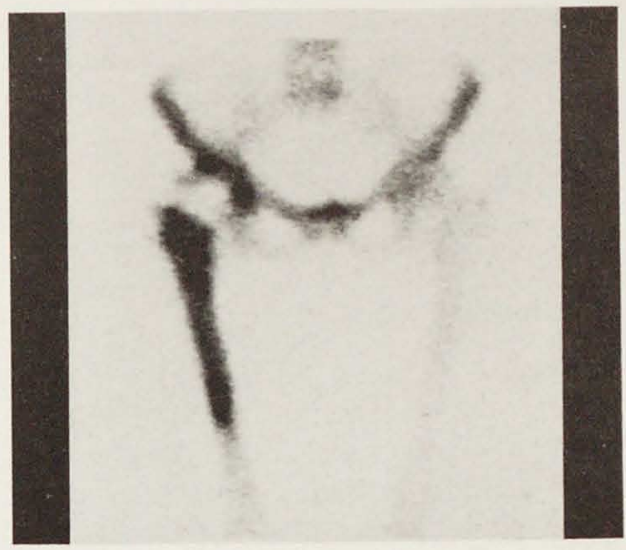

Fig. 109

Technetium bone-scan six weeks after hydroxylapatite coated total hip replacement. Elevated activity over proximal and distal femoral stem section as well as acetabular area.
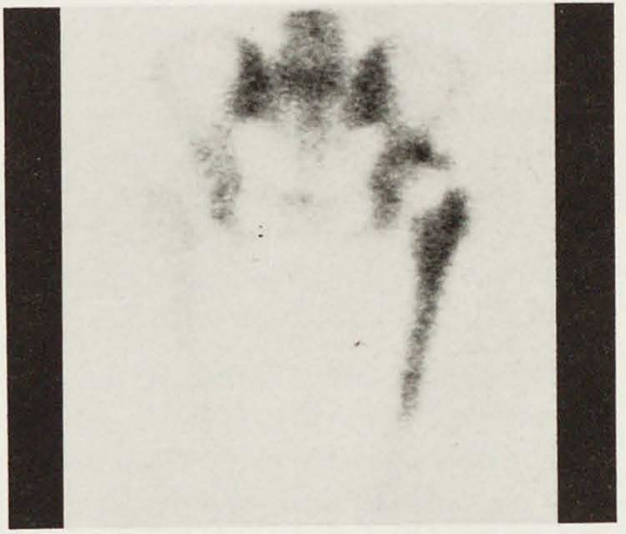

Fig. 111

Technetium bone-scan of human hydroxyl-apatite coated total hip replacement, six months after surgery. Moderate activity discernible over hydroxylapatite coated part of prosthesis in proximal stem area. 


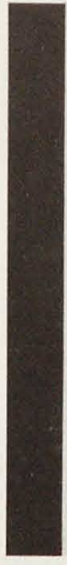

Fig. 112

Technetium bone-scan of human hydroxyl-apatite coated total hip replacement, nine months after surgery. Moderate activity discernible over hydroxylapatite coated part of prosthesis in proximal stem area, with moderate persisting activity over femoral stem tip.
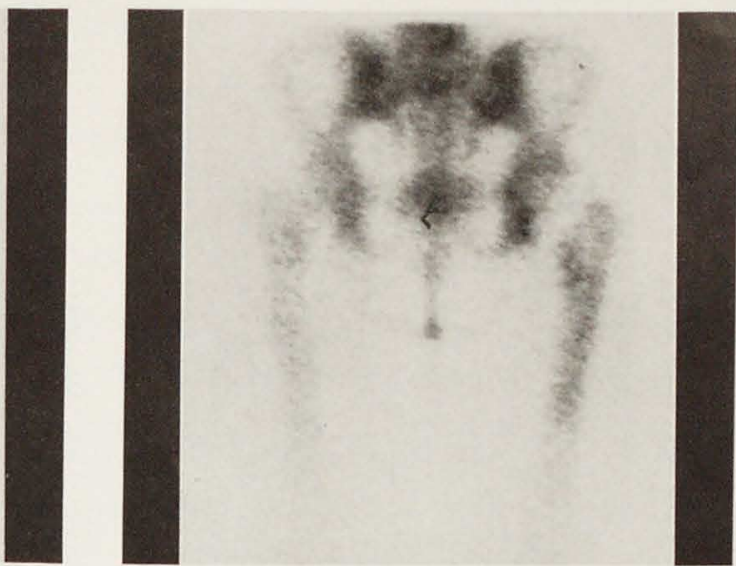

Fig. 113

Technetium bone-scan of human hydroxyl-apatite coated total hip replacement, nine months after implantation. Activity confined to hydroxyl-apatite coated part of prosthesis in proximal stem area, without persisting activity over femoral stem tip.

Uptake in the acetabular region was generally less elevated as compared to the femoral side and followed the classical pattern of early (two weeks postoperatively) high uptake. Six months after surgery uptake around the acetabular component was only slightly elevated as compared to the non-operative side. There were no hot spots or other specific focal accumulations of technetium. After one year, uptake over the prosthesis was only slightly elevated in comparison to the normal side. Some patients had higher localized uptakes of technetium over small areas of non-symptomatic peri-articular calcifications, persisting until one year after surgery.

\section{IIIm-Indium-labeled leucocyte scans}

All performed scans with $111 \mathrm{~m}$-indium-labeled leukocytes were normal within two weeks after operation. There were no focal accumulations of $111 \mathrm{~m}$-indium activity on any part of the hip area. After these negative findings the routine use of $111 \mathrm{~m}$-indium-labeled leucocyte scans was discarded.

\section{Problem cases}

There were two clinical cases with unsatisfactory results, both caused by postoperative peri-articular calcifications. Both patients are in Charnley's category $\mathrm{C}$, indicating coexistent disease impairing hip performance. They will be discussed below.

\section{case I}

One 49 year old female patient, with a spinal fusion and severe backache caused by thoraco-lumbar kypho-scoliosis ( $116^{\circ}$ angle) of neuro-muscular origin, developed spinal convulsions that caused a bilateral fracture of the hip. Both a displaced medial neck fracture of the left hip and a central acetabular fracture of the right hip, were treated with 
a hydroxyl-apatite coated total hip implant (Fig. 114 and 115). The left hip developed extensive peri-articular calcifications and became ankylosed. Ten months after primary surgery, the calcifications were removed and the non-coated screw-cup replaced with a cemented one (Fig. 116). She regained 85 degrees of flexion in the left hip, the right hip having a full range of motion. Although both hips are painfree, she still experiences her original backaches.

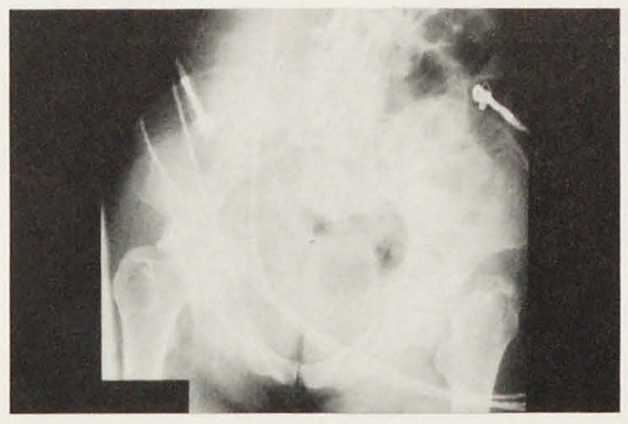

Fig. 114

Bilateral hip fractures, preoperative radiograph.

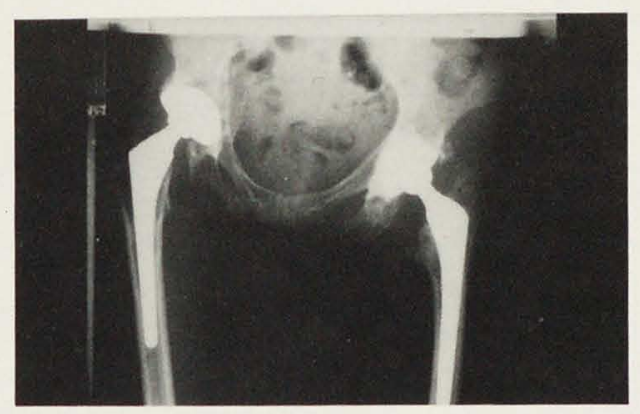

Fig. 116

Same case as previous after removal of ectopic bone and change of acetabular non-coated screw-ring to cemented cup.

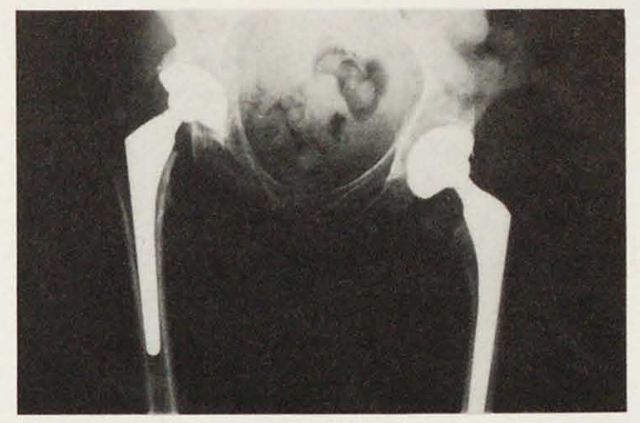

Fig. 115

Same case as previous six weeks after surgery, periarticular calcifications of left hip.

case 2

A 39 year old male patient with Bechterews disease had a long standing history of hip complaints with a completely ankylosed hip. The hip was replaced with a hydroxyl-apatite coated prosthesis. Although the patient was known to be at risk for ectopic bone formation, he was not allowed to use any preventive Indocid ${ }^{\circledR}$ medication because of a simultaneous history of Crohns disease with many gastro-intestinal complications. The alternative diphosphonates proved ineffective in preventing peri-articular calcifications. Although the patient is completely painfree, there is only 45 degrees of flexion in the hip, impairing certain activities of daily living. 
case 3

A 59 year old lady with advanced osteo-arthrosis of the hip was treated with a hydroxylapatite coated total hip implant. The postoperative X-ray showed a correct position of the implant, but at first follow-up, six weeks after surgery, she had some complaints in the upper thigh. The X-ray showed a fissure in the proximal femur with subsidence of the femoral stem of approximately one centimeter (Fig. 117). She was treated for another six weeks on restricted weight-bearing and the fissure/fracture healed uneventfull. Six months after surgery, there is a Harris hip score rating of 96, with a normal range of motion and an asymptomatic one centimeter leg length discrepancy.

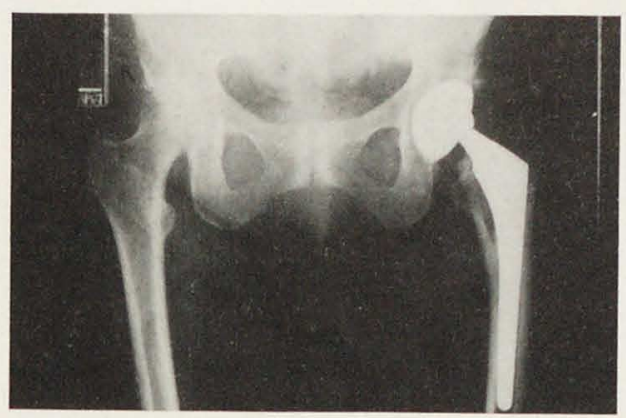

Fig. 117

Post-operative femoral fissure/fracture with stem subsidence.

\section{Discussion}

The early clinical follow-up data indicate that functional recovery after hydroxyl-apatite coated total hip arthroplasty is rapid. Especially striking is the almost complete absence of pain after the operation. The first six weeks after operation, patients use crutches and thereby partially unload the affected extremity. Once full-weight-bearing commences, patients remain free of pain and this continues afterwards up to longest follow-up of eighteen months. There is not a single patient with the characteristics of "mid-thigh pain" persisting after the third month of surgery. There were two patients sustaining some ischiadic type of pain in the whole leg for up to three or four months postoperatively. There was no relation of this pain with joint-loading or otherwise using the hip. They possibly relate to a chronic low-back pain syndrome in one instance. The cause in the other patient is less clear, it could theoretically relate to some partial damage of the ischiadic nerve. Both complaints resolved spontaneously within four months after operation. Another patient had lateral-thigh pain early after surgery, disappearing in the fourth month after surgery.

Recovery of activities of daily life will take more time. As seen from table VII, there is a continuing rise in performance up to the sixth month, after which the ratings level off. Recovery of muscular force and range of motion of the hip joint is even more slowly and will probably still continue after the sixth month. Especially the rotations of the hip start to recover only from the third or fourth month after operation. The average gain in postoperative flexion of the hip is at least 30 degrees. Almost all flexion deformities have disappeared within six weeks after operation. 
Based on the curves, it can be extrapolated that there will be a slight but definitive further gain in results between the sixth and twelfth months after operation. Recovery of activities of daily life, muscular force, and restoration of range of motion in the hip relate to a certain extent on factors other than the characteristics of the implant.

Preoperative life style and range of motion determine to a significant degree the postoperative speed of functional recovery, provided that the implant itself permits painless performance.

Complications in this still small series are comparable to other non-cemented hip surgery. Generally, the frequency of intra-operative fissures and or fractures tends to be somewhat higher as compared to cemented hip surgery. This is caused by the necessary tight initial mechanical fit of the implant in the bone, stretching the bone sometimes to its ultimate limit of tolerance. A high standard of surgical skill is required to accomplish an optimum initial primary fit between implant and bone. Judicious preoperative planning in conjunction with good instruments and trial implants can prevent many problems.

The incidence of clinical significant peri-articular calcifications does not appear to be any different from other hip replacement surgery. Both patients with ectopic bone formation constituted special categories with increased risk for such calcifications. There is no patient with osteo-arthrosis with clinical significant ectopic bone formation, although there are several with minor calcifications around the hip but with normal ranges of motion in the hip.

The most obvious difference between hydroxyl-apatite coated total hip prostheses and other more conventional cementless hip implants appears to be the short recovery period and the absence of "mid-thigh pain" in the hydroxyl-apatite group. Although the numbers are still small at the time of this writing ( 80 implants by the author, with many others in a prospective multicenter clinical follow-up study), the difference appears already significant. In a comparable study in progress (Capello 1987), using the same prosthesis, but then without hydroxyl-apatite coating, in a similar population with the same follow-up criteria, the percentage of mid-thigh pain was between 15-20 percent at one year. For other types of non-cemented hip implants, comparable figures of $20-25$ percent midthigh pain are known (Herbert 1987). Although it is difficult to compare heterogenous groups of patients, it is still tempting to do so. Using the Chi-square test with a significance level of $\mathrm{P}<0.05$, a consecutive group of 50 patients with one case of mid-thigh pain represents already a significant difference. If the percentage of persisting "mid-thigh pain" would be in the order of four percent, as is for instance known for certain types of cemented hip implants, groups of at least one hundred patients are necessary to prove any differences with current non-cemented hip implants and even much larger groups to reveal any differences with cemented hip implants. Carefully designed prospective studies are needed to further clarify differences with our current generation of implants.

The radiological findings at follow-up appear similar to those of the experimental studies. The complete absence of radio-lucent lines around the hydroxyl-apatite coated part of the implant suggests a very close apposition of bone against the prosthesis, while the increase in bone density with condensation of endosteal bone to bone of almost cortical structure indeed suggests stress-transfer through the coating. Because this process of endosteal bone 
densification is limited to the coated part of the prosthesis: it is accentuated near the termination of the coating at the femoral mid-stem and stops were the coating ends, may prove that this process is the predominant effect of the hydroxyl-apatite coating in conjunction with a good prosthetic cross-sectional geometry. It also suggests that loadcarrying is limited to the hydroxyl-apatite coated part of the femoral component. Additionally, there were no signs of stress-shielding in the proximal femur up to current follow-up. Although this can not be excluded for longer follow-up, the presence of proximal densification of trabecular bone appears reassuring in the prevention of stressshielding osteo-porosis. The proximal presence only of the coating is important in this respect.

The distal part of the femoral stem does not appear to take any major load-bearing. Console formation near the stem-tip was not seen, although regularly formation of a very thin radio-lucent line was seen around the distal part of the non-coated femoral stem. This can be explained by the difference in modulus of elasticity between bone and metal of the implant. This phenomenon will be even enhanced because of the strong fixation of the implant in the proximal femoral stem area. Under these circumstances of proximal fixation of the implant, the deflection of the stiffer implant against the bone by loadbearing forces will be the largest near the femoral stem tip. Because the cortical bone of the femur is more elastic as compared to the metal of the implant (even made out of titanium) some micro-motion is inevitable between implant stem-tip and bone. This is not necessarily accompanied by significant load-bearing in this part of the implant, as evidenced by the absence of console formation below the stem tip. The micromotion between implant and bone, through mismatch of modulus of elasticity between metal and bone, will cause a very localized zone of bone resorption, which appears as a thin radiolucent line around the distal non-coated parts of the femoral stem on the radiographs.

The radiological findings are in agreement with the results of the technetium bone-scans. Early postop, technetium uptake is equally distributed over the coated as well as the noncoated part of the femoral component. Usually, technetium uptake is slightly higher over the non-coated distal part of the femoral stem. Therefore, this increase is probably more related to the surgical procedure of intra-medullary reaming during implantation than to the hydroxyl-apatite coating itself. This reaming causes a diffuse osteoblastic response in the bone-marrow to heal the bone and thereby integrate the implant into the bone. Within two or three weeks after surgery, these high technetium uptakes gradually diminish. After two months, technetium uptake becomes more confined to the area of hydroxyl-apatite coating with a very even distribution of radio-nuclide activity over this part of the femur. This is a sign of the bone-bonding process being limited to the area of hydroxyl-apatite coating. Once technetium uptake becomes settled to its near preoperative low level, the process of bony integration of the implant is almost finished, although there may be a more prolonged period of bone remodelling at a much lower rate of bone activity.

Nevertheless, in some cases, there is a second area near the stem-tip with localized marginal elevation in uptake persisting after the sixth month. This could be in agreement with the regularly observed process of radio-lucent line formation around the non-coated distal part of the femoral stem tip, as described before. It can probably be explained by a localized process of bone resorption, which is generally accompanied by an elevated bone 
formation as well. This localized increase in bone metabolism is possibly caused by the micro-motion between implant and bone, which may be caused by the relative differences in stiffness between implant and bone and enhanced by the strong proximal fixation of the implant, as described earlier. More refined analysis of radio-nuclide findings is contingent on continuing research.

Initially, the high postoperative technetium uptakes caused some difficulties in interpretation, because they were also higher as compared to cemented or non-coated cementless implants of similar design. Several additional investigations did not reveal any pathologic cause for these high uptakes.

Indium-labeled leucocyte scans were all normal within two weeks after operation. An inflammatory factor is therefore not probable. Although some patients appeared to have a somewhat higher rate of postoperative increase in blood sedimentation rate (BSE), the overall differences in routine blood chemistry were not significantly different from routine hip surgery (Berglund ea. 1979). More specifically, alkaline phosphatase, transaminase and creatinine estimations were normal at all times after all surgery. Because blood loss with cementless hip surgery usually is somewhat higher than with cemented hip surgery, any difference in rise of BSE could be caused by the higher number of required units of blood transfusion.

Based on the short term results, with all their inherent limitations, the conclusion can already be drawn that hydroxyl-apatite coated total hip replacement adds to a significantly improved postoperative result as compared to other cementless implants, the largest gain being absence of pain. This in itself allows a higher activity level to be reached in a shorter period of time.

Although short term results appear very satisfactory, we have to await longer term followup. Nevertheless, there are some prognostic factors to the life expectancy of hydroxylapatite coated implants. Radiology and bone scintigraphy provide tools for the determination of mechanical and biological stability at the implant-bone interface. Radiography of hydroxyl-apatite coated prostheses, after one year of implantation and longer, show excellent quality of bone around implants without any radio-lucent line formation around coated parts of implants. Often there is an increase in bone mass around the prosthesis. Bone scintigraphy using $99 \mathrm{~m}$-technetium shows a low uptake with very even distribution of radio-nuclide activity in relation to the bone mass over the entire area of the hydroxyl-apatite coating. Both radiological and scintigraphic findings point to a stable equilibrium between implant and bone. Therefore, the life expectancy of hydroxylapatite coated implants should theoretically be satisfactory, clinical practice being the final answer.

This study represents current knowledge on hydroxyl-apatite coated implants and is only a starting point for further developments. Future research must focus on the consequences of the biological bonding concept on implant design. Careful monitoring of clinical, radiological and bone scintigraphic results will certainly provide important clues for further developments. Refinements can be expected with respect to implant geometry as well as the area of hydroxyl-apatite coating on the femoral stem. For instance, the persisting tip activity with bone scintigraphy in conjunction with the radiological findings 
of radio-lucent line formation around the distal non-coated part of the femoral stem probably proves that implant stiffness in the distal stem area is too high. Although there are no relevant accompanying clinical signs, it still proves that current implant geometry can and should be improved.

Additionally, the rather localized increase in bone density near the termination of the hydroxyl-apatite coating in the mid-stem section of the implant, as seen on many radiographs, probably proves that the current circular ending of the hydroxyl-apatite coating at the mid-part of the femoral stem is less than ideal.

At the time of this writing, there is a prototype of an improved femoral stem design with increased flexibility of its distal stem section, with retention of the characteristics of the proximal stem area $\left(\right.$ Omniflex $\left.{ }^{\circledR}\right)$ (Fig. 118). The decrease in stiffness is accomplished through a decrease in stem cross-section in its distal part. Optimal fit of the stem tip with the endosteal part of the femoral bone is still maintained with the aid of separate cylindrical tip components that fit on the stem-tip and adjust the implant to the actual internal diameter of the femoral shaft in that specific area. Because these tip components have a strict cylindrical geometry with polished surface, they are not capable of any load-bearing. The distal termination of the hydroxyl-apatite coating can possibly be higher on the lateral aspect of this stem. The coating should be retained in the proximal lateral stem area, because this is an important area of stress-transfer with hydroxyl-apatite coated implants.

Although short term clinical results of hydroxyl-apatite coated implants are very promising, the most important criterion for success is still their longterm performance. Ten years of clinical follow-up is the minimum required for proper comparison with our current generation of hip prostheses. This is beyond the scope of this work and the subject of future research.

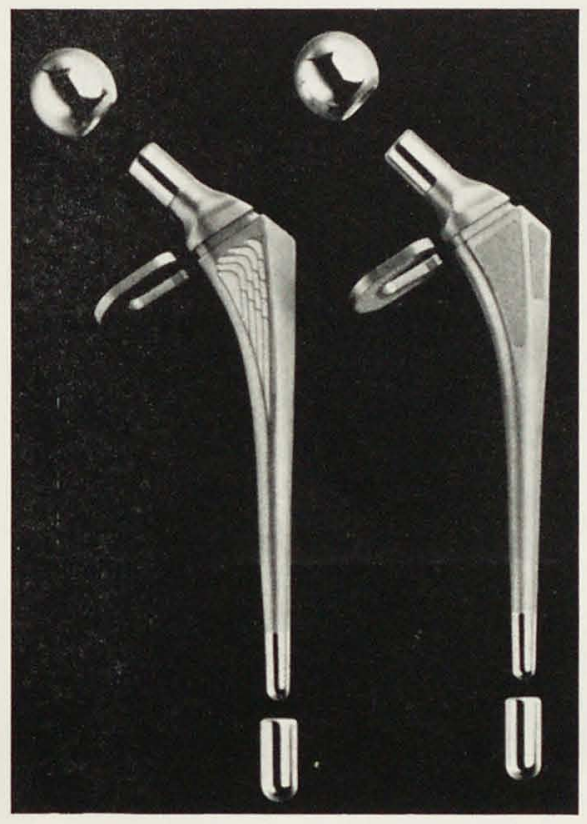

Fig. 118

Improved stem design with more flexible distal stem section and modular stem tips (Omniflex $\left.{ }^{\circledR}\right)$. 


\section{Chapter ten}

\section{General conclusions and summary}

An important cause for inadequate longterm performance of artificial hip implants relates to the limited biocompatibility characteristics of the implant materials in use. Implant materials, according to their biological response in the body, are identified as (1) biotolerant, (2) bioinert or (3) bioactive materials. Biotolerant materials are, for example, bone cement or stainless steel, that invoke a fibrous tissue response when implanted in the body. Titanium is an example of a bioinert material that can effect a close approximation to bone, but still can not effect bonding to bone. Bioactive materials have free calciumand phosphate compounds at their surface, which enables them to effect a chemical bonding to bone (chapter one).

Hydroxyl-apatite is the most important representative of the bioactive calcium-phosphate ceramics. The chemical and physical characteristics of hydroxyl-apatite are discussed. There is abundant evidence in the literature that sintered hydroxyl-apatite is very well incorporated into and acquires very strong bonds with living bone. Furthermore it does not undergo any significant biodegradation once bonded to the bone. Although the static mechanical strength of sintered hydroxyl-apatite is comparable to that of cortical bone, its major drawback for use in load-bearing applications is its high susceptibility to fatigue failure under tensile loading. This has limited thus far the use of hydroxyl-apatite in surgery to non-weight-bearing applications (chapter two).

The use of a composite material, consisting of a hydroxyl-apatite coating on a metal substrate can theoretically overcome fatigue problems. Titanium is especially well suited for this purpose.

Hydroxyl-apatite coated titanium combines the strength of the titanium metal core with the biological surface characteristics of hydroxyl-apatite. Through the use of a hightemperature plasma-spraying procedure, a strong and durable chemical bonding can be obtained between coating and metal (chapter three).

The chemical analysis of the hydroxyl-apatite coating and the determination of its static and fatigue strength indicate that the coating-substrate bonding strength is at an acceptable level to justify further investigations (chapter four).

A human cadaveric implantation and retrieval study has confirmed that the risk of loosening of the hydroxyl-apatite coating from the titanium substrate during implantation is negligible (chapter five).

The elementary biological and mechanical interface characteristics of hydroxyl-apatite coatings to bone have been determined in a transcortical plug implant study in dogs. In this plug study, an ultimate bone-bonding shear strength of $30 \mathrm{MPa}$ after three months was established. After six weeks there is already a strong bonding of the implant to the bone. The recorded values are probably sufficient to cope with the stress levels encountered under clinical implantation conditions. The histological results confirm the close bonding between hydroxyl-apatite coating and bone, similar to that known for sintered hydroxyl-apatite (chapter six). 
A canine total hip replacement model studied the behaviour of hydroxyl-apatite coated implants under conditions of significant mechanical load-bearing. There proves to be a striking difference in biological response between non-coated and hydroxyl-apatite coated implants, both having the same initial pressfit fixation in bone. Non-coated prostheses have persisting micro-motion at the implant-bone interface as seen on radiographs with radio-lucent line formation.

Additionally, bone scintigrams using $99 \mathrm{~m}$-technetium show three point hot spot accumulation of $99 \mathrm{~m}$-technetium as a sign of instability at the implant-bone interface. Histologically, the non-coated prostheses are surrounded by a fibrous tissue membrane and are easily extracted from the femur at any postoperative time interval. Hydroxylapatite coated prostheses acquire a strong chemical bone-bonding within a time interval of three to six weeks over their entire surface area. In the histological sections, there is good bonding between hydroxyl-apatite coating and bone. Not only periosteal tissue and mature bone, but also many osteoblasts and complete Haversian systems have direct endings on the surface of the coating. At two years follow-up, there is excellent bone quality with the hydroxyl-apatite coated prostheses in the direct vicinity of the implantbone interface, although there are sometimes signs of proximal stress-shielding osteoporosis. This appears related to rigid distal stem fixation of implants and it is a sign that full length biological coatings on femoral stems are probably not advisable. The condition of the coating at two years follow-up appears good. Apart from some stress-shielding osteo-porosis, radiographs after two years show excellent quality of bone near the boneimplant interface in comparison to the non-coated side, while bone scans using $99 \mathrm{~m}$ technetium show a very even distribution of activity over the entire area of hydroxylapatite coating. Both the radiological, scintigraphic and histological findings point to a stable equilibrium at the implant-bone interface of hydroxyl-apatite coated prostheses. Especially the short time interval in which a hydroxyl-apatite coating can achieve a strong bone bond and the fact that close apposition between bone and coating at surgery is not necessary to achieve good results are striking advantages. No adverse tissue response has evolved thus far. The transfer of the chemical bone bonding concept to human applications appears safe (chapter seven).

One dog had an infectious complication. Histological investigation revealed fragmentation and dissolution of the hydroxyl-apatite coating, the implant became loose. Theoretically, this is conceivable, because the hydroxyl-apatite coating is chemically unstable at $\mathrm{pH}$-levels of five or less. Under infectious conditions there often is a lowered tissue $\mathrm{pH}$-value (chapter eight).

As a practical outcome of this study, a human total hip replacement prosthesis was developed with a proximal hydroxyl-apatite coating on the femoral stem and a hydroxylapatite coated acetabular screw-ring. General considerations on indications for operation, operative technique when different from current techniques, after-treatment and early case data on clinical performance of first human implantations are detailed (chapter nine).

The early clinical follow-up data indicate that functional recovery after hydroxyl-apatite coated total hip arthroplasty is rapid. Especially striking is the almost complete absence of pain or other discomfort after the operation, when compared to more conventional 
cementless implants. Radiological and radio-nuclide investigations suggest that bony fixation and load-bearing are limited to the hydroxyl-apatite coated part of the implant. The radiological finding of endosteal bone densification, and thereby increase in bone mass, offers good perspectives for longterm implant performance. Based on the short term results, with all their inherent limitations, it is apparent that hydroxyl-apatite coated total hip replacement adds to a significantly improved postoperative result as compared to other cementless implants. The largest gain in performance is complete absence of mid-thigh or other pain, which in itself allows a higher activity level to be reached.

The main emphasis in this study has been the experimental work on the use of hydroxylapatite coated implants in orthopaedic surgery. Although both animal and clinical studies thusfar have shown an excellent performance of hydroxyl-apatite coated prostheses, the most important criterion for success is the longterm clinical performance of these implants. Ten years of clinical follow-up is the minimum required for proper comparison with our current generation of hip prostheses. This is beyond the scope of this work and the subject of continuing research. 



\section{Chapter eleven}

\section{Samenvatting}

Een probleem in de chirurgie van kunstgewrichten is nog altijd het funktioneren van deze implantaten op lange termijn. Blijkens literatuur gegevens bedraagt het revisie percentage van totale heupprothesen na 10 jaar tot $30 \%$. Waar wereldwijd 300.000 totale heup implantaties per jaar worden verricht, waarvan circa 12.000 in Nederland, betekent dit een aanzienlijke morbiditeit. Gangbare methoden van implantaat fixatie berusten op het gebruik van botcement. Botcement of polymethyl-methacrylaat is een twee-komponenten kunsthars, waarmee een mechanische verankering in de trabekelstruktuur van het omliggende bot wordt bereikt. Hechting van botcement aan bot zelf treedt niet op. Belangrijke oorzaken van falen van kunstmatige gewrichts-implantaten zijn de achteruitgang in de tijd van de mechanische eigenschappen van botcement samen met de thermo-chemische neven-effekten van botcement op het omliggende bot tijdens en na implantatie. Hierdoor treedt vaak botresorptie op, waarna bindweefsel de plaats van bot inneemt. Begin van loslating van de prothese is het gevolg. De minder goede biocompatibiliteit van de gebruikte implantatie materialen speelt dus een belangrijke rol in het loslatings-proces van kunst gewrichten. Meer biocompatibele implantatie materialen zouden hierin verbetering kunnen brengen. Een gangbare klassifikatie van biomaterialen gaat uit van hun biologische reaktie in het lichaam. Zo onderscheidt men, met toenemende graad van biocompatibiliteit, biotolerante, bioinerte en bioactieve implantatie materialen. Biotolerante materialen, als bijvoorbeeld botcement, worden door bindweefsel van het lichaam afgeschermd, doordat zij voor het lichaam irritatieve stoffen aan hun oppervlak afgeven. Bioinerte materialen, als bijvoorbeeld titanium, zijn chemisch in hoge mate neutraal ten opzichte van het lichaam en roepen daardoor een zodanig lage biologische afweer reaktie op, dat zij onder optimale mechanische omstandigheden in direkt botkontakt kunnen treden.

Hechting van bioinerte materialen aan bot treedt evenwel niet op, zodat bij gebruik in kunstmatige gewrichtsprothesen nog steeds van principes van mechanische verankering gebruik moet worden gemaakt. Bioaktieve materialen kunnen door de aanwezigheid van vrije calcium- en phosphaat-verbindingen aan hun oppervlak een chemische en biologische verbinding met bot aangaan. De belangrijkste vertegenwoordiger in deze groep bioaktieve materialen is hydroxyl-apatiet (hoofdstuk één).

Toepassing van hydroxyl-apatiet in de implantaat-chirurgie lijkt veelbelovend, aangezien het zowel in chemische struktuur als crystallografische opbouw identiek is aan het apatiet van het lichaamseigen bot. Biokompatibiliteits problemen zijn daardoor niet te verwachten. Uit de literatuur is bekend, dat apatiet implantaten een zeer hechte verbinding van chemische aard met bot kunnen aangaan en voorts, dat de stabiliteit van het materiaal in het lichaam op lange termijn goed is. Hoewel de statisch mechanische sterkte van hydroxyl-apatiet goed is, zijn de vermoeidheids karakteristieken van massieve apatiet implantaten helaas slecht. Hierdoor moet de toepassing vooralsnog beperkt blijven tot implantaten, die niet aan mechanische belasting zijn blootgesteld. Toepassing als heup- of knie-prothese is hiermee uitgesloten (hoofdstuk twee). 
Door het aanbrengen van een dunne hydroxyl-apatiet coating op een metalen onderlaag kan een komposiet materiaal worden verkregen, dat de gunstige biologische eigenschappen van apatiet aan zijn oppervlak kombineert met de sterkte van een metalen kern. Titanium is hiertoe in het bijzonder geschikt. De coating wordt aangebracht door middel van een plasma-spray procédé, waarmee een hechte en duurzame chemische verbinding tussen apatiet en titanium kern wordt verkregen (hoofdstuk drie).

Om de toepasbaarheid voor orthopaedische implantaten te onderzoeken zijn verscheidene onderzoekingen verricht. Bij materiaalkundig onderzoek blijken zowel de statische sterkte als vermoeidheids karakteristieken van hydroxyl-apatiet coatings goed te zijn. Voorts blijken in de praktijk gangbare methoden van sterilisatie van implantaten geen nadelige effekten op de materiaalsterkte te hebben (hoofdstuk vier).

Ook na kadaver implantatie met navolgende extraktie van prothesen, wordt geen signifikante beschadiging van de hydroxyl-apatiet coating gezien. De fysische eigenschappen van de coating bieden dus een voldoende veiligheidsmarge bij klinische toepassing (hoofdstuk vijf).

In een onderzoek met plugimplantaten bij honden zijn de elementaire mechanische en biologische eigenschappen van de botverbinding met hydroxyl-apatiet coatings nader onderzocht zonder gebruikmaking van mechanische retentie technieken.

Röntgenologisch bestaat reeds na zes weken een goede botingroei van de pluggen. Bij onderzoek op de testbank, door middel van "push-out" tests, wordt na zes weken een botverbinding in schuifsterkte van 48 Megapascal gemeten met een eindwaarde van 59 Megapascal, d.w.z $580 \mathrm{~kg} / \mathrm{cm}^{2}$, vanaf de derde maand. Histologisch onderzoek laat een direkte botverbinding zien, niet alleen met periost en osteocyten, maar ook met jonge osteoblasten. Dit onderstreept de goede biokompatibiliteit van het materiaal. De resultaten van het plugonderzoek bewijzen dat apatiet gecoate implantaten een botverbinding kunnen aangaan, die vergelijkbaar is met wat bekend is voor gesinterde apatiet implantaten (hoofdstuk zes).

Hoewel dit onderzoek het grote potentieel van apatiet coatings voor biologische fixatie van implantaten aantoont blijft een beperking, dat de pluggen tijdens de ingroeifase niet aan mechanische krachten waren blootgesteld, wat de resultaten kan flatteren. Daarom is in een derde onderzoek de toepassing als belast implantaat onderzocht. Bij vijftien honden zijn apatiet gecoate totale heupprothesen van een eigen ontwerp ingebracht met overigens identieke ongecoate protheses als controle in de andere heup. Beide implantaties volgens gangbare pressfit-technieken. Reeds na drie weken is er een duidelijk verschil ten gunste van de gecoate prothesen vast te stellen.

Ongecoate kontrole-protheses kunnen op ieder willekeurig ogenblik uit het femur geëxtraheerd worden. Röntgenologisch zijn na één jaar en langer osteolytische reakties rond de prothesesteel zichtbaar, terwijl ook scintigrafisch onderzoek met $99 \mathrm{~m}$-technetium aanwijzing geeft voor tenminste mikro-beweeglijkheid ter plaatse van de bot-implantaat overgang. Histologisch bezien, zijn de ongecoate protheses door een bindweefsel membraan omgeven met daarbij tekenen van botresorptie op het implantaat-bot grensvlak. 
In tegenstelling hiermee zijn de apatiet gecoate implantaten na drie tot zes weken niet gemakkelijk meer te extraheren.

$\mathrm{Bij}$ poging daartoe treden fissuren en fracturen in het omliggende bot op. De röntgenfoto na één jaar toont een gaaf interface zonder enige lijnvorming en voorts een goede botregeneratie tot op ruime afstand van het implantaat. Met name na twee jaar werd bij één hond signifikante stress-shielding phenomenen vastgesteld in de vorm van verlies van kortikale dikte en spongiosering van de corticalis. Dit lijkt vooral in verband te staan met rigide fixatie rond de prothese punt. Biologische coatings over de volle lengte van de prothese lijken daarom minder aanbevelenswaardig. Botscintigrafie met $99 \mathrm{~m}$-technetium toont een diffuse lage aktiviteit over het hele prothese bereik met gelijkmatige verdeling van de radio-aktiviteit over het gebied van de coating. De aktiviteit is min of meer lineair met de hoeveelheid bot rond de prothese. Er zijn geen gebieden met hot spots of andere tekenen, die op instabiliteit ter plaatse van de prothese-bot verbinding kunnen wijzen. In de histologie wordt een direkte botverbinding gezien met de prothese, waarbij ook botdefekten tot op twee à drie millimeter afstand binnen zes weken met bot worden opgevuld. Het karakteristieke bot-implantaat interface bestaat uit een botplaat op het prothese-oppervlak, die reeds na twee à drie weken aanwezig is en in verloop van de tijd steeds dikker wordt en opvult tot massief bot. De aanhechtende bottrabekels vertonen daarbij in de regel een specifieke oriëntatie onder invloed van belastings-krachten. Daarbij neemt de botdichtheid naar het implantaat-oppervlak toe. Voorts bestaat een rijke osteoblasten-aktiviteit tot op het prothese-oppervlak en zolang er botombouw is eveneens osteoclasten-aktiviteit. De apatiet coating van de prothese raakt hierdoor volledig geïntegreerd in het omliggende bot. Na twee jaar is de kwaliteit van de coating in de histologie goed en is de dichtheid van het bot uitstekend.

Conclusie van dit onderzoek is, dat ook onder omstandigheden van zware mechanische belasting op de prothese, met apatiet gecoate implantaten een hechte botverbinding mogelijk is. Doordat de prothese nu niet alleen compressie-belasting kan opnemen, maar ook trek- en scheer-krachten, wordt een veel meer natuurlijke botbelasting rond de prothese bereikt. Voorts treedt door de goede osteoconductiviteit van apatiet een maximale opvulling op van altijd aanwezige vullings-defekten rond een cementloze prothese. Het lijkt aannemelijk, dat de belangrijkste winst van apatiet coatings voor cementloze implantaten niet alleen haar goede biologische fixatie is, maar evenzeer haar goede osteoconductiviteit. Hierdoor wordt de prothese in korte tijd over vrijwel haar volledige oppervlak door een stevige botmantel omgeven. Micro-beweeglijkheid wordt hierdoor voorkomen en de stress op de overigens ook goede prothese-botverbinding blijft binnen aanvaardbare grenzen. Met name de snelheid waarmee de botverbinding wordt opgebouwd en het feit, dat geen volledig exacte pasvorm tussen implantaat en bot nodig is om goede resultaten te verkrijgen bieden duidelijke voordelen ten opzichte van poreuze metaal coatings. Aangezien voorts geen nadelige biologische reakties werden vastgesteld, lijkt toepassing van apatiet gecoate implantaten bij de mens zinvol (hoofdstuk zeven).

Eén hond met een apatiet gecoate prothese kreeg een diepe wondinfektie van de heup. Bij histologisch onderzoek werd fragmentatie en oplossing van de coating gezien, waardoor de prothese grotendeels los ging laten. Ook theoretisch is dit te verwachten, aangezien apatiet chemisch instabiel is bij een $\mathrm{pH}$ waarde van minder dan vijf. Bij infekties bestaat vaak een weefselacidose (hoofdstuk acht). 
Als resultaat van dit onderzoek is een humane titanium totaalprothese voor de heup ontwikkeld met apatiet coating. Daarbij wordt van een anatomisch gevormde femoraal komponent uitgegaan, waarbij de coating om biomechanische redenen alleen proximaal wordt aangebracht. Als acetabulum komponent wordt een sferische schroefcup gebruikt. Indikaties, operatietechniek, voorzover afwijkend van gangbare technieken en nabehandeling worden besproken.

Eerste humane implantaties zijn vanaf 1986 door de auteur verricht. Hoewel klinische follow-up nog kort is en daarmee uiteraard nog geen voorspelling over lange termijn funktioneren toelaat, is zelfs met deze beperkte groep al duidelijk dat met apatiet gecoate prothesen een aanmerkelijk sneller funktioneel herstel kan worden verkregen, dan met meer gebruikelijke cementloze heupprothesen. In het bijzonder valt de hoge mate van pijnvrijheid op, wat een optimale revalidatie bevordert. Röntgen- en isotopen-onderzoek tonen aan, dat botingroei en daarmee krachtoverdracht in de prothese inderdaad beperkt blijven tot dat deel van de prothese, dat met hydroxyl-apatiet is gecoat. De toename van botmassa vanwege het beschreven proces van endostale botverdichting blijkens röntgenfoto's en de lage diffuse verdeling van radio-aktiviteit op bot scintigrammen ter plaatse van de hydroxyl-apatiet coating van de prothese zijn een teken van mechanische en biologische stabiliteit ter plaatse van de prothese-bot verbinding. Hierdoor bestaat een goede prognose voor lange termijn funktioneren van hydroxyl-apatiet gecoate implantaten (hoofdstuk negen).

De nadruk bij het verrichte onderzoek ligt evenwel voornamelijk bij het dierexperimentele werk. Hierbij kan worden vastgesteld, dat met apatiet gecoate implantaten een aanzienlijke verbetering in zowel aard als sterkte van de bot-implantaat verbinding kan worden verkregen. $\mathrm{Na}$ één tot twee jaar implantatie onder zowel experimentele als klinisch-humane omstandigheden blijkt de botkwaliteit beter dan bij andere methoden van implantaat-fixatie. Dit kan de levensduur van de prothese alleen maar ten goede komen.

Het belangrijkste kriterium voor succes van kunstmatige gewrichtsimplantaten blijft evenwel hun lange termijn funktioneren. Tien jaar klinische follow-up is wel de minimaal benodigde tijd om goede vergelijkingen met gangbare methoden van implantaatfixatie te kunnen maken. Dit valt buiten het bestek van dit werk en is onderwerp van voortgaand onderzoek. 


\section{Chapter twelve}

\section{Resume}

Le fonctionnement à long terme des prothèses articulaires reste un problème de la chirurgie de ces implants. Selon les données de la littérature le pourcentage total de réintervention des prothèses totales de hanche après dix ans s'élève à $30 \%$. Puisque dans le monde entier 300.000 implantations de prothèses totales de hanche sont effectuées chaque année, dont environ 12.000 aux Pays-Bas, cela signifie une morbidité considérable. Les méthodes courantes de fixation de l'implant reposent sur l'emploi de ciment osseux, le polyméthyle-méthacrylate, une résine synthétique à deux composants, grâce auquel on obtient un ancrage mécanique dans la structure trabéculaire de l'os environnant. Il ne se produit pas d'adhésion du ciment osseux à l'os lui-même. Les causes principales d'échec des implants de prothèses articulaires sont la dégradation dans le temps des propriétés mécaniques du ciment osseux associée aux effets secondaires thermo-chimiques du ciment osseux sur l'os environnant pendant et après l'implantation. C'est pourquoi il survient souvent une résorption osseuse; du tissu conjonctif prend alors la place de l'os. Un début de descellement de la prothèse en est la conséquence. La moins bonne biocompatibilité des matériaux d'implantation utilisés joue donc un rôle important dans le processus de descellement des prothèses articulaires de hanche. Des matériaux d'implantation à meilleure biocompatibilité pourraient ici apporter une amélioration. Une classification courante des biomatériaux se base sur leur réaction biologique dans le corps. Ainsi l'on distingue, par degré croissant de biocompatibilité des matériaux d'implantation biótolérants, bioinertes et bioactifs. Des matériaux biotolérants, comme par exemple le ciment osseux, sont toujours séparés du corps par du tissu conjonctif, parce qu'ils dégagent en surface des substances irritantes pour le corps. Des matériaux bioinertes, comme par exemple le titane, sont chimiquement en grande partie neutres par rapport au corps et déclenchent ainsi une réaction biologique de rejet tellement basse qu'ils peuvent entrer en contact directement avec l'os dans des circonstances mécaniques optimales. La suture des matériaux bioinertes à l'os ne se produit pas cependant, de sorte qu'il faut toujours se servir de principes d'ancrage mécanique lors de leur utilisation dans les prothèses articulaires artificielles. Les matériaux bioactifs peuvent réaliser une liaison chimique et biologique avec l'os grâce à la présence de radicaux libres de calcium et de phosphate en surface. Le réprésentant le plus important de ce groupe de matériaux bioactifs est l'hydroxyle d'apatite (chapitre un).

L'application de l'hydroxyle d'apatite dans la chirurgie des implants semble prometteuse, puisque cette substance est identique à l'hydroxyle d'apatite de l'os du corps aussi bien par sa struture chimique que par sa constitution crystallographique. On ne doit donc pas s'attendre à des problèmes de biocompatibilité. On sait par la littérature que des implants d'hydroxyle d'apatite peuvent réaliser une liaison de nature chimique très solide avec l'os et de plus, que la stabilité à long terme du matériau dans le corps est bonne. Bien que la solidité mécanique statique de l'hydroxyle d'apatite soit bonne, les caractéristiques de fatigabilité des implants en apatite massives sont hélas mauvaises. Pour cette raison il faut jusqu'à présent en réserver l'application aux implants qui ne sont pas exposés à une charge mécanique. 
L'utilisation dans les prothèses de hanche ou de genou en est donc exclue (chapitre deux).

En applicant un mince revêtement d'hydroxyle d'apatite sur un support métallique on peut obtenir un matériau composite, qui combine les propriétés biologiques favorables de l'hydroxyle d'apatite en surface avec la solidité du noyau métallique. Le titane est particulièrement adapté à cette fin. On applique le revêtement à l'aide d'un procédé de spray au plasma, grâce auquel on obtient une liason chimique solide et durable entre l'apatite et le noyau en titane (chapitre trois).

Diverses expérimentations ont été effectuées pour en étudier l'applicabilité dans les implants orthopédiques. Lors de l'étude des propriétés des matériaux les caractéristiques de résistance statique ainsi que la fatigabilité des revêtements d'hydroxyle d'apatite paraissent offrir une bonne marge de sécurité par rapport aux forces qui apparaissent habituellement à l'endroit de la liaison implant-os. De plus les méthodes courantes de stérilisation des implants paraissent en pratique ne pas avoir d'effets néfastes sur la solidité du matériau (chapitre quatre).

On ne voit pas non plus de dégradation significative du revêtement d'hydroxyle d'apatite lors d'implantations sur le cadavre suivies de l'extraction des prothèses. Les propriétés physiques du revêtement offrent ainsi une marge de sécurité suffisante pour l'application clinique (chapitre cinq).

Dans une étude portant sur des implants en chevilles chez des chiens on a examiné plus précisement les propriétés mécaniques et biologiques élémentaires de la liaison osseuse avec les revêtements d'hydroxyle d'apatite, sans utiliser de techniques de rétention mécanique. A la radiographie il existe dès après six semaines un bon envahissement osseux des chevilles. A l'examen au banc d'essai, à l'aide de tests de "push-out", on mesure après six semaines une liaison osseuse en force de cisaillement de 48 mégapascal avec une valeure finale de 59 mégapascal, soit $580 \mathrm{~kg} / \mathrm{cm}^{2}$, à partir du troisième mois. L'examen histologique montre une liaison osseuse directe, non seulement avec du périoste et des ostéocytes, mais également avec de jeunes ostéoblastes. Ceci souligne la bonne biocompatibilité du matériau. Les résultats de l'étude portant sur les chevilles prouvent que les implants recouverts d'hydroxyle d'apatite peuvent contracter une liaison osseuse comparable à ce qui est connu des implants d'hydroxyle d'apatite frittée (chapitre six).

Bien que cette étude démontre le grand potentiel des revêtements d'hydroxyle d'apatite pour la fixation biologique des implants, il reste une limitation: les chevilles n'ont pas été exposées à des forces mécaniques pendant la phase d'envahissement, ce qui peut flatter les résultats. C'est pourqoi on a examiné l'application comme implant en charge dans une troisième étude.

On a implanté des prothèses totales de hanche recouverte d'hydroxyle d'apatite de propre fabrication chez quinze chiens, des prothèses non recouvertes mais par ailleurs identiques servant de contrôle sur l'autre hanche. Les deux implantations ont été effectuées selon les techniques courantes de "pressfit". Dès après trois semaines on constate une différance évidente en faveur des prothèses recouvertes. Les prothèses de contrôle non recouvertes peuvent être extraites du fémur à n'importe quel moment. A la radiographie on voit apparaître des réactions ostéolytiques autour de la queue de la prothèse trois mois et plus après l'insertion. Avec la scintigraphie osseuse il y a des signes de micromobilité des 
prothèses non recouvertes deux ans après l'insertion. Histologiquement, les prothèses non recouvertes sont entourées d'une membrane de tissu conjonctif et il y a des signes de résorption osseuse à l'interface implant-os.

Par contre on ne peut plus extraire facilement les implants recouvertes d'hydroxyle d'apatite après trois à six semaines. Si l'on essaie il se produit des fissures et des fractures dans l'os environnant. La radiographie après un an montre un interface intact sans formation de trait ainsi qu'une régénération osseuse jusqu'à une ample distance de l'implant. La scintigraphie osseuse avec des prothèse recouvertes en apatite montre une activité de l'os également distribué autour du fémur sans signes de micromobilité ou de descellement après deux ans d'implantation. A l'histologie, on voit une liaison osseuse directe avec la prothèse, tandis que des lacunes osseuses sont également comblées par du tissu osseux jusqu'à deux ou trois millimètres de distance dans les six semaines.

L'interface os-implant caractéristique consiste en une plaque osseuse sur la surface de la prothèse, qui est déjà présente après deux à trois semaines et s'épaissit de plus en plus au cours du temps et se remplit d'os massif. En outre les trabécules osseux qui s'y attachent montrent généralement une orientation spécifique sous l'influence des forces de charge. De plus la densité osseuse augmente vers la surface de l'implant. Il existe également une riche activité ostéoblastique jusque sur la surface de la prothèse et aussi longtemps qu'il y a une activité osseuse il existe aussi une activité ostéoclastiqe. Le revêtement en apatite de la prothèse se trouve ainsi complètement intégré dans l'os environnant. Après deux ans la qualité du revêtement est toujours aussi bonne à l'histologie et la densité de l'os est excellente. La conclusion de cette étude est qu'on peut avoir une liaison osseuse résistante dans les implants recouverts d'hydroxyle d'apatite également dans dans des circonstances de lourde charge mécanique sur la prothèse. Du fait que la prothèse peut non seulement supporter des charges de compression mais également des forces de traction et de cisaillement, on atteint une mise en charge osseuse beaucoup plus naturelle autour de la prothèse. De plus grâce à la bonne ostéoconductivité de l'hydroxyle d'apatite il se produit un remplissage optimal des lacunes toujours présentes autour d'une prothèse non cimentée. On peut envisager que l'apport le plus important des revêtements d'hydroxyle d'apatite dans les implants non cimentées est non seulement sa bonne fixation biologique, mais tout autant sa bonne ostéoconductivité. Grâce à cela la prothèse est entourée en peu de temps par une solide chape osseuse sur presque toute sa surface. La micromobilité est ainsi empêchée et le stress sur la liaison os-prothèse, qui est par ailleur bonne, reste dans des limites acceptables. Puisque la charge articulaire de ce modèle de prothèse totale de hanche chez le chien est au moins trois fois plus élevée que chez l'homme, et que l'on n'a pas constaté de réactions biologiques néfastes, l'application à l'homme des implants recouverts d'apatite semble possible (chapitre sept).

Chez un chien ayant une prothèse recouverte d'apatite, il y eut une infection profonde de la plaie de la hanche. A l'examen histologique il y avait fragmentation et dissolution du revêtement, ce qui explique le détachement de la prothèse. On peut s'attendre théoriquement à cela, puisque l'apatite est chimiquement instable si le pH est inférieur à cinq. Il existe souvent une acidose tissulaire dans les infections (chapitre huit).

A la suite de cette étude on a mis au point une prothèse humaine totale de hanche en titane avec revêtement d'apatite. 
On est parti d'un composant fémoral à forme anatomique, ou le revêtement n'a pu être appliqué que dans la région proximale pour des raisons biomécaniques. On utilise une coupe à vis sphérique comme composant acétabulaire. Les indications, la technique opératoire, pour autant qu'elles different des techniques courantes et le traitement postopératoire sont analysés. Les prémières implantations humaines ont été effectuées à partir de 1986.

Bien que le suivi clinique soit encore bref et ne permette donc aucune prévision sur le fonctionnement à long terme, il est déja évident même avec ce groupe limité que l'on obtient un rétablissement fonctionnel nettement plus rapide avec les prothèses de hanche recouvertes d'apatite qu'avec de nombreuses prothèses de hanche usuelles non cimentées. On remarque en particulier le haut degré d'absence de douleur, ce qui favorise une rééducation optimale (chapitre neuf).

L'accent repose cependant dans l'étude effectuée sur le travail expérimental chez l'animal. On a pu établir ainsi que l'on peut obtenir une amélioration considérable de la nature et de la solidité de la liaison os-implant avec les implants recouvertes d'apatite. Après deux ans d'implantation chez les chiens, la qualité osseuse se révèle être meilleure que dans les autres méthodes de fixation de l'implant. Cela ne peut qu'influencer favorablement la durée de vie de la prothèse. Le critère le plus important de succès des prothèses articulaires artificielles n'en demeure pas moins leur fonctionnement à long terme. Un suivi clinique de dix ans est bien le temps minimum nécessaire pour faire des comparisons valables avec les méthodes courantes de fixation de l'implant. Ceci tombe en dehors du cadre de ce travail et est le sujet d'une étude ultérieure. 


\section{Literature references}

1. Albrektsson T, Albrektsson B: Osseointegration of bone implants. Acta Orthop Scand 58: 567-577, 1987.

2. Albright JA: Bone, physical properties. in Albright JA, Brand RA, The scientific basis of orthopaedics, pp 135-184. Appleton, New York, ISBN 0-8385-8503-5, 1979.

3. Almby $\mathbf{B}$, Hierton $\mathbf{T}$ : Total hip replacement: 10 year follow-up of an early series. Acta Orthop Scand 53: 398-406, 1982.

4. Andersen AC: The beagle as an experimental dog. Hematology pp. 261-280. Iowa State Univ press, ISBN 0-8138-016-9, 1970.

5. Anderson JM: Biomaterials biocompatibility and the macrophage. Biomaterials 5: 5-10, 1984.

6. Anderson RC, Cook SD, Weinstein AM, Haddad RJ: An evaluation of skeletal attachment to LTI pyrolytic carbon, porous titanium and carbon-coated porous titanium implants. Clin Orthop 182: 242-257, 1984.

7. Austin RT: Granulomatosis of bone from high density poly-ethylene. Injury 13:414-418, 1982.

8. Barth E, Ronnigen H, Solheim LF: Comparison of ceramic and titanium implants in cats. Acta Orthop Scand 56: 491-495, 1985.

9. Berglund B, Bergstrom K: Serum enzymes after hip joint surgery. Acta Orthop Scand 50: 671-673, 1979.

10. Black J: Systemic effects of biomaterials. Biomaterials 5: 11-18, 1984.

11. Blitterswijk CA van, Grote JJ, Kuypers W, Daems WTh, Groot K de. Macropore Tissue Ingrowth: A Quantitative and Qualitative Study on Hydroxyl-apatite Ceramic. Biomaterials 7: 137-144, 1986.

12. Bobyn JD, Engh CA: Human histology of the bone-porous metal implant interface. Orthopedics 7: 1410-1421, 1984.

13. Bobyn JD, Pilliar RM, Cameron HU, Weatherley GC: The Optimum Pore Size for the Fixation of Porous Surfaced Metal Implants by the Ingrowth of Bone. Clin Orthop Rel Res 50:263-270, 1980.

14. Boogaard AEJM, Weidema WF: Anti-microbial prophylaxis in canine surgery. J Small Anim Pract 26: 257-266, 1985.

15. Boyd AD: Effects of polymethyl-methacrylate on rabbit articular chondrocytes in monolayer culture. Clin Orthop 189: 279-293, 1984.

16. Brand RA, Pedersen DR, Yoder SA: How definition of loosening affects the incidence of loose total hip reconstructions. Clin Orthop Rel Res 210: 185-191, 1986.

17. Branemark PI ea. Osseointegrated implants. Almqvist and Wiksell International, Stockholm 1977

18. Brill W, Katthagen BD: Die Grenzschicht zwischen Hydroxyl-apatit Keramik und neugebildeten Knochen im Raster-elektronen Mikroskop. Z Orthop 125: 183-187, 1987.

19. Brown IW, Ring PA: Osteolytic Changes in the Upper Femoral Shaft Following Porouscoated Hip Replacement. J Bone Joint Surg 67-B: 218-221, 1985. 
20. Brown TD, Ferguson AB: The development of a computational stress analysis of the femoral head. J Bone Joint Surg 60A: 619-629, 1978.

21. Buchert PK, Vaughn BK, Mallory TH, Engh CA, Bobyn JD: Excessive Metal Release Due to Loosening and Fretting of Sintered Particles on porous-coated Hip Prostheses: Report of two Cases. J Bone Joint Surg 68-A: 606-609, 1986.

22. Bunshah RF, editor. Deposition Technologies for Films and Coatings. Noyes Publications, Park Ridge, NY USA ISBN 0-8155-0906-5, 1981.

23. Cameron HU, Pilliar RM. Macnab I: The effect of movement on the bonding of porous metal to bone. J Biomed Mater Res 7: 301-309, 1973.

24. Cameron HU: The results of early clinical trials with a microporous coated metal hip prosthesis. Clin Orthop 165: 188-191, 1982.

25. Capello WN: Personal communications, 1987.

26. Capello WN: Preoperative planning of total hip arthroplasty. Instr Cours Lect 35: 249-257, 1986.

27. Carlsson L, Röstlund T, Albrektsson B, Albrektsson T: The effect of surgical fit on implant fixation by appositional bone growth. in press, Acta Orthop Scand 1988.

28. Carter DR, Spengler DM: Mechanical Properties and Composition of Cortical Bone. Clin Orthop Rel Res 135: 192-217, 1978.

29. Chandler HP, Reineck FT, Wixson RL, Mccarthy JC. Total Hip Replacement in Patients Younger than 30 Years Old. J Bone Joint Surg 63A:1426-1429, 1981.

30. Charnley J, Cupic Z: The nine and ten year results of the low-friction arthroplasty of the hip. Clin Orthop 95: 9-25, 1973.

31. Charnley J: Low friction arthroplasty of the hip. Springer, Heidelberg, 1983.

32. Chen PQ, Turner PM, Ronnigen $\mathbf{H}$, Galante J: A canine cementless total hip prosthesis model. Clin Orthop 176: 24-33, 1983.

33. Christel P, Meunier A, Leclerq S, Bouquet Ph, Buttazoni B: Development of a carboncarbon hip prosthesis. J Biomed Mater Res 21: 191-218, 1987.

34. Christoffersen J: Dissolution of calcium hydroxyl-apatite. Calcif Tissue Inter 33: 557-565, 1981.

35. Collis DK: Cemented Total Hip Replacement in Patients who are less than Fifty Years Old. J Bone Joint Surg 66-A: 353-359, 1984.

36. Cook SD, Georgette FS, Skinner HB, Haddad RJ: Fatigue Properties of Carbon and Porous-coated Ti-6Al-4V Alloy. J Biomed Mater Res 18: 497-512, 1984.

37. Cook SD, Kay JF, Anderson TRC, Harding AF, Reynolds MC, Jarcho M: Variables Affecting the Interface Strength and Histology of Hydroxyl-apatite Coated Implant Surfaces. 12th Annual meeting of the Society for Biomaterials. Minneapolis, 1986

38. Cook SD, Reynolds MC, Whitecloud TS, Routman AS, Harding AF, Kay JF, Jarcho M: Evaluation of Hydroxylapatite Graft Materials in Canine Cervical Spine Fusions. Spine 11: 305-309, 1986.

39. Cook SD, Walsh KA, Haddad AJ: Interface Mechanics and Bone Growth into Porous CoCr-Mo Alloy Implants. Clin Orthop Rel Res 193: 271-280, 1985. 
40. Cornell CN, Ranawatt CS: Survivorship Analysis of Total Hip Replacements. Results in a Series of Active Patients who were less than Fifty Years Old. J Bone Joint Surg 68A: 14301434, 1986.

41. Crowninshield RD, Brand RA, Johnston RC: The Effects of Walking Velocity and Age on Hip Kinematics and Kinetics. Clin Orthop Rel Res 132: 140-144, 1978.

42. Crowninshield RD, Brand RA, Johnston RC, Milroy JC: An Analysis of Femoral Component Stem Design in Total Hip Arthroplasty. J Bone Joint Surg 62A: 68-78, 1980.

43. Crugnola A, Radin E: Polymeric debris in synovium after total joint replacement: histological identification. J Bone Joint Surg 59A: 68-72, 1977.

44. Currey JD: The mechanical properties of bone. Clin Orthop Rel Res 73: 210-231, 1970.

45. Davidge RW: Structural degradation of ceramics. Biomaterials 5: 37-41, 1984.

46. Denissen $\mathbf{H W}$, Groot $\mathbf{K}$ de, Klopper PJ: Tissue response to dense apatite implants in rats. J Biomed Mater Res 14: 713-721, 1980.

47. Denissen HW, Veldhuis AAH, Rejda BV: Dental root implants as abutments for crown and bridge work and dolder prosthesis. Ned Tijdschr Tandheelk 90: 89-95, 1983.

48. Denissen HW: The Linkage between Apatite Implant Material and Living Bone. Ultramicroscopy 5: 124-128, 1980.

49. Dorr L, Takei GK, Conathy JP: Total Hip Arthroplasties in Patients Less than Forty-five Years Old. J Bone Joint Surg 65A: 474-479, 1983.

50. Draenert K, Ruediger J: Histomorphologie des Knochen-Zement Kontaktes. Chirurg 49: 276-285, 1978.

51. Driessen AA, Klein CPAT, de Groot K: Preparation and some properties of sintered Bwhitlockite. Biomaterials 3: 113-116, 1982.

52. Driessens FCM: Probable phase composition of the mineral in bone. $Z$ Naturforsch $35 c$ : 357-362, 1980.

53. Ducheyne P, Aernoudt E, De Meester P, Martens M, Mulier JC, VanLeeuwen D: Factors Governing the Mechanical Behavior of the Implant- Porous Coating - Trabecular Bone Interface. J Biomech 11: 297-308, 1978.

54. Ducheyne P, Hench LL, Kagan A, Martens $\mathbf{M}$, Mulier JC: Short term Bonding Behaviour of Bioglas Coatings on Metal Substrate. Arch Orthop Traumat Surg 94: 155-160, 1979.

55. Ducheyne $\mathbf{P}$, Martens $\mathbf{M}$, Burssens $\mathbf{A}$ : Materials, Clinical and Morphological Evaluation of Custom-made Bioreactive Glass-coated Canine Hip Prostheses. J Biomed Mater Res 18: 1017-1030, 1984

56. Ducheyne P, Raemdonck W van, Heughebaert JC, Heughebaert $M$ : Structural Analysis of Hydroxyl-apatite Coatings on Titanium. Biomaterials 7: 97-104, 1986.

57. Editorial JAMA: Total hip-joint replacement in the United States. JAMA 248: 1817-1821, 1982.

58. Editorial JBJS: The need for a standardized system for evaluating results of total hip surgery. J Bone Joint Surg 67A: 511-512, 1985.

59. Endler M, Endler TA, Zielinski C: Influence of hip arthroplasty upon chemotactic behaviour of leucocytes. Acta Orthop Scand 53: 795-798, 1982. 
60. Engh CA, Bobyn JD, Glassman AH: Porous-coated Hip Replacement: The Factors Governing Bone Ingrowth, Stress Shielding, and Clinical Results. J Bone Joint Surg 69-B: 45-55, 1987.

61. Eriksson RA: Assessment of bone viability after heat trauma. Scand J Plast Reconstr Surg $261-268,1984$.

62. Evans FG: Mechanical properties of bone. Thomas, Springfield, 1973.

63. Feenstra L, Groot $\mathbf{K}$ de: Medical use of calcium-phosphate ceramics; in Bioceramics of calcium-phosphate. Ed. K de Groot, CRC Press, Boca Raton, 1983.

64. Feith R, Slooff TJJH, Kazem I, van Rens TJG: Strontium 87-m bone scanning for the evaluation of total hip replacement. J Bone Joint Surg 58B: 79-83, 1976.

65. Feith R: Side effects of acrylic cement, implanted into bone. Acta Orthop Scand, suppl 161, 1975.

66. Flatley TJ, Lynch KL, Benson M: Tissue Response to Implants of Calcium Phosphate Ceramic in the Rabbit Spine. Clin Orthop 179: 246-252, 1983.

67. Frost HM: A determinant of bone architecture, the minimum effective strain. Clin Orthop 175: 286-292, 1983.

68. Fuchs GA, Deutscher K: Glass-ceramic Coated Implants. Arch Orthop Trauma Surg 98: $121-126,1981$.

69. Gaechter A, Alroy J, Andersson GBJ, Galante J ea. Metal carcinogenesis. J Bone Joint Surg 59A: 622-624, 1977.

70. Galante JD: Corrosion related failures in metallic implants. Clin Orthop 86: 237-244, 1972.

71. Gates EI, Harris WH: Comparative fatigue behaviour of different bone cements. Clin Orthop 189: 294-299, 1984.

72. Geesink RGT, Groot K de, Klein CPAT: Bone bonding to apatite coated implants. J Bone Joint Surg 70B: 17-22, 1988.

73. Geesink RGT, Groot K de, Klein CPAT: Chemical implant fixation using hydroxyl-apatite coatings. Clin Orthop 225: 147-170, 1987.

74. Geesink RGT, Groot K de, Klein CPAT: Implant fixation using hydroxyl-apatite coatings "1987 Sir John Charnley Award" by "Hip Society", presented at the annual meeting of the Hip Society, January 21 1987, San Francisco.

75. Gitelis S, Chen P, Andersson GBJ, Galante JO, Rostoker W, Andriacchi TP: The Influence of Early Weight-bearing on Experimental Total Hip Arthroplasties in Dogs. Clin Orthop 169: 291-302, 1982.

76. Goldring SR, Schiller AL, Roelke M, Harris WH: The Synovial-like Membrane at the Bone-cement Interface in Loose Total Hip Replacements and its Proposed Role in Bone Lysis. J Bone Joint Surg 65A: 575-584, 1983.

77. Goodship AE: Functional adaptation of bone to increased stress. J Bone Joint Surg 61A: 539-546, 1979.

78. Green SA: The effect of methylmethacrylate on phagocytosis. J Bone Joint Surg 57A: 583, 1975.

79. Griss P, Werner E, Heimke G, Raute-Kreinsen U: Comparative Experimental Investigations with Bioglass (L.L. Hench) and AL2O3 Ceramic Coated with Mod. Bioglass.Ii. Arch Orthop Trauma Surg 92: 199-210, 1978. 
80. Griss P, Werner E, Heimke G, Buchinger R: Vergleichende experimentelle Untersuchungen an Bioglas, A12O3-Keramik und mit Bioglas beschichteter Keramik. Arch Orthop Unfall-Chir 90: 15-27, 1977.

81. Gristina AG, Rovere GD: An in vitro study of bacterial response to inert and reactive metals and methylmethacrylate. J Biomed Mater Res 10:273-281, 1976.

82. Gristina AG: Bacterial adherance to biomaterials and tissue. J Bone Joint Surg 67A: 264-273, 1985.

83. Groot K de, Geesink RGT, Klein CPAT, Serekian P: Plasma-sprayed Coatings of Hydroxylapatite. J Biomed Mat Res, 21: 1375-1381, 1987.

84. Groot K de, Putter C de, Sillevis Smit PAE, Driessen AA: Mechanical Failure of Artificial Teeth Made of Dense Calcium-hydroxyl-apatite. Science of Ceramics 11: 433-437, 1981.

85. Groot K de: Bioceramics Consisting of Calcium-phosphate Salts. Biomaterials 1: 47-49, 1980.

86. Groot K de: Ceramics based on calcium-phosphates, in: Ceramics in Surgery, Ed Vincenzini P. Elsevier, Amsterdam, 1983.

87. Groot K de: Calcium-phosphate ceramics: Their current status. In Contemporary Biomaterials, pp. 477-492. Ed. JW Boretos, Noyes Publ, Park Ridge NJ, ISBN 08-155-09804, 1984.

88. Gross U, Brandes J, Strunz V, Bab I, Sela J: The Ultrastructure of the Interface between a Glass Ceramic and Bone. J Biomed Mater Res 15: 291-306, 1981.

89. Grote JJ, Groot K de: Use of sintered hydroxyl-apatite in middle ear surgery. ORL 43: 248$252,1981$.

90. Gruen TA, Amstutz HC: Effects of laminations and blood entrapment on the strength of acrylic bone cement. Clin Orthop 119: 250-255, 1976.

91. Haddad RJ, Cook SD, Thomas KA: Current concepts review: Biological fixation of porouscoated implants. J Bone Joint Surg 69A: 1459-1466, 1987.

92. Halawa $M$, Lee A, Ling RSM: The Shear Strength of Trabecular Bone from the Femur and some Factors Affecting the Shear strength of the Cement-bone Interface. Arch Orthop Traum Surg 92: 19-30, 1978.

93. Halley DK, Wroblewski BM: Long-term results of low-friction arthroplasty in patients 30 years of age or younger. Clin Orthop 211: 43-50, 1986.

94. Hansen LB, Kromann B, Baekgaard N: Uncemented two-component femoral prosthesis for the hip joint: a 50-month follow-up study. Clin Orthop 208: 182-187, 1986.

95. Harms J, Mausle E: Tissue Reaction to Ceramic Implant Material. J Biomed Mater Res 13: $67-88,1979$.

96. Harris WH, White RE, McCarthy JC, Walker PS, Weinberg EH: Bony ingrowth fixation of the acetabular component in canine hip joint arthroplasty. Clin Orthop 176: 7-11, 1983.

97. Harris WH, McCarthy JC, O'Neill DA: Femoral Component Fixation using Contemporary Techniques of Femoral Cement Fixation. J Bone Joint Surg 64A: 1063-1067, 1982.

98. Harris WH, White RE JR: Socket fixation using a metal-backed acetabular component for total hip replacement: a minimum five-year follow-up. J Bone Joint Surg 64-A: 745-748, 1982. 
99. Harris WH: Advances in total hip arthroplasty, the metal-backed acetabular component. Clin Orthop 183: 4-11, 1984.

100. Heck DA, Nakajima I, Kelly PJ, Chao EY: The effect of load-alteration on the biological and biomechanical performance of a titanium fiber-metal segmental prosthesis. J Bone Joint Surg 68A: 118-126, 1986.

101. Hedley AK, Clarke IC, Kozinn SC, Coster I, Gruen T, Amstutz HC: Porous ingrowth fixation of the femoral component in a canine surface replacement of the hip. Clin Orthop 163: 300-311, 1982.

102. Hench LL, Pantano CG, Buscemi PJ, Greenspan DC: Analysis of bioglass fixation of hip prostheses. J Biomed Mater Res 11: 267-282, 1977.

103. Herbert P: National Swedish study on cementless total hip replacement. Harris hip course, Boston 1987. to be published.

104. Holmes RE, Bucholz RW, Mooney V: Porous Hydroxyapatite as a Bone- Graft Substitute in Metaphyseal Defects: A Histometric Study. J Bone Joint Surg 68-A: 904-911, 1986.

105. Hoogendoorn HA, Renooij W, Akkermans LMA, Visser W, Wittebol P: Longterm Study of Large Ceramic Implants (Porous Hydroxyl-apatite) in Dog Femora. Clin Orthop 187: 281 $288,1984$.

106. Huiskes R: Finite-element analysis of acetabular reconstruction, non-cemented acetabular cups. Acta Orthop Scand 58: 620-625, 1987.

107. Huiskes R, Weinans H, Grootenboer HJ, Dalstra M, Fudala B, Sloof TJ: Adaptive bone remodelling theory applied to prosthetic design analysis. J Biomech 20: 1135-1150, 1987.

108. Huiskes R: Biomechanics of bone-implant interactions. In: Frontiers in biomechanics, Ed. Schmid-Schönbein GW, Woo SLY, Zweifach BW, pp 246-262, Springer, Berlin 1986.

109. Huiskes R, Vroemen W: A standardized finite-element model for routine comparative evaluation of femoral hip prostheses. Acta Orthop Belg 52: 258-261, 1986.

110. Huiskes R, Nunamaker D: Local stresses and bone adaptation around orthopaedic implants. Calcif Tissue Int 36: 110-117, 1984.

111. Huiskes R, Chao EYS: A survey of finite-element analysis in orthopaedic biomechanics: the first decade. J Biomech 16: 385-409, 1983.

112. Huiskes R: Some fundamental aspects of human joint replacement. Acta Orthop Scand, suppl 185, 1979.

113. Ilstrup DM, Coventry MB: Factors influencing the results in 2012 total hip arthroplasties. Clin Orthop 95: 250-262, 1973.

114. Itami Y, Akamatsu N, Tomita Y, Nagai M, Nakajima I: A clinical study of the results of cementless total hip replacement. Arch Orthop Trauma Surg 102: 1-10, 1983.

115. Jarcho M, Kay JF, Gumaer KI, Doremus RH, Drobeck HP: Tissue, Cellular and Subcellular Events at a Bone-ceramic Hydroxyl-apatite Interface. J Bioengineering I: 79-92, 1977.

116. Jarcho M: Calcium Phosphate Ceramics as Hard Tissue Prosthetics. Clin Orthop 157: 259 $278,1981$.

117. Kallenberger A, Mathys R, Mueller W: Untersuchngen der Gewebe-verträglichkeit von Hydroxyl-apatit an kultivierten Fibroblasten. Hefte Unfallheilk 165: 71-74, 1983. 
118. Katz JL: Present and potential biomedical applications of composite materials technology. In: Contemporary Biomaterials, pp. 453-475. Ed. Boretos, Noyes Publ, Park Ridge NJ, ISBN 08-155-0980-4, 1984.

119. Kawamura M, Iwata H, Sato K, Miura T: Chondro-osteogenetic Response to Crude Bone Matrix Proteins Bound to Hydroxyapatite: Clin Orthop 217: 281-292, 1987.

120. Kay JF, Uratsuji N, Stern LS, Stuhlberg DN, Manley M: Hydroxyl-apatite coatings applied to implants subject to functional loading. Transactions of the 13 th annual meeting of the Society for Biomaterials. New York, Waldorf Astoria, 1987.

121 Kay JF, Jarcho M, Logan G, Liu ST: The Structure and Properties of Hydroxyl-apatite Coatings on Metal. 12th Annual Meeting of the Society for Biomaterials. Minneapolis 1986

122. Kay ME, Young RA: Crystalstructure of hydroxyl-apatite. Nature 204: 1050, 1964.

123. Kent JN, Block MS, Kay J, Jarcho M, Finger IM: Hydroxyl-apatite Coated and Noncoated Dental Implants in Dogs. 12th Annual meeting of the Society for Biomaterials. Minneapolis 1986

124. Kent JN, Jarcho M: Correction of alveolar ridge deficiencies with non-resorbable hydroxylapatite. JADA 105: 993-1001, 1982.

125. Klein CPAT, Abe Y, Hosono H, Groot K de: Different Calcium Phosphate Ceramics Implanted in Rabbit Cortical Bone. An Interface Study. Biomaterials 5: 362-364, 1984.

126. Klein CPAT, Driessen AA, Groot K de: Relationship between Degradation Behaviour of Calcium Phosphate Ceramics and their Physical-chemical Characteristics and Ultrastructural Geometry. Biomaterials 5: 157-160, 1984.

127. Klein CPAT, Driessen AA, Groot K de, Hooff A van den: Biodegradation behaviour of various calcium-phosphate materials in bone tissue. J Biomed Mater Res 17: 769-784, 1983.

128. Klein CPAT, Groot $\mathrm{K}$ de, $\mathrm{Kamp} \mathbf{G}$ van: Activation of complement $\mathrm{C}_{3}$ by different calciumphosphate powders. Biomaterials 4: 181-184, 1983.

129. Klein CPAT, Kamp $\mathbf{G}$ van, Groot $\mathbf{K}$ de, Vermeyden JPW: Interaction of some seroproteins with hydroxyl-apatite and other materials. J Biomed Mater Res 14: 705-712, 1980.

130. Klein CPAT, Lubbe HBM van der, Driessen AA, Groot $\mathrm{K}$ de: Biodegradation behaviour of various calcium-phosphate materials in subcutaneous tissue. In: Ceramics in Surgery, Ed. Vincenzini, Elsevier, Amsterdam, 1983.

131. Klein CPAT: Calcium Phosphate Implant Materials and Biodegradation. Thesis, Free University, Amsterdam, 1983.

132. Krajewski A, Monticelli G: The behaviour of apatite-based ceramics in relation to the critical $1150-1250^{\circ} \mathrm{C}$ temperature range. Biomaterials 5: 105-108, 1984.

133. Krause WR, Krug W, Eng B, Miller J: Strength of the Cement-bone Interface. Clin Orthop 163: 290-299, 1982.

134. Kummer FJ, Rose RM: Corrosion of titanium/cobalt-chromium alloy couples. J Bone Joint Surg 65A: 1125-1126, 1983.

135. Lacefield WR: The Coating of Hydroxyl-apatite onto Metallic and Ceramic Implants. 12th Annual Meeting of the Society for Biomaterials. Minneapolis, 1986

136. Lacout JL, Assarane J, Trombe JC: Some reactions between phospho-calcium hydroxyapatite and titanium-oxide. C.R. Acad Sc Paris 298: 173-175, 1984. 
137. Lange DR: The Mechanical Bonding of Methyl-methacrylate to Cancellous Bone. J Bone Joint Surg 61A: 254-256, 1979.

138. Lanyon LE: Mechanically adaptive bone remodelling. J Biomech 15: 141-154, 1982.

139. Lees S, Davidson CL: The role of collagen in the elastic properties of calcified tissues. J Biomech 10: 473-480, 1977.

140. Linder L: Monomer leakage from polymerizing acrylic bone cement. Clin Orthop 119: 242$249,1976$.

141. Linder L: Reaction of bone to the acute chemical trauma of bone cement. J Bone Joint Surg 59A: 82-87, 1977.

142. Lord G, Bancel P: The Madreporic Cementless Total Hip Arthroplasty: New Experimental Data and a Seven-year Clinical Follow-up Study. Clin Orthop 176: 67-76, 1983.

143. Lunceford EM, Moderator JR, Collier JP, Engh C, Gustilo RB, Hedley AK: Symposium: Biologic Fixation of Porous-coated Implants in Total Hipreplacement. Contemp Orthop 9: 53-89, 1984.

144. Magerl F, Schenk W, Muller W: Klinische Erfahrungen mit geformten Hydroxylapatitblöcken. In Biomaterialien und nahtmaterial, Ed. H Rettig, Springer, Heidelberg, 1983.

145. Manley MT, Kotzar G, Stern LS, Wilde A: Effects of Repetitive Loading on the Integrity of Porous Coatings. Clin Orthop 217: 293-302, 1987.

146. Mattingly DA, Hopson CN, Kahn A III, Giannestras NJ: Aseptic loosening in metalbacked acetabular components for total hip replacement: a minimum five-year follow-up. J Bone Joint Surg 67-A: 387-391, 1985.

147. McBeath AA: Circumferential and axial strain in the proximal femur. Clin Orthop 150: 301 $305,1980$.

148. McKellop H, Clarke I, Markolf K, Amstutz H: Friction and Wear Properties of Polymer, Metal and Ceramic Prosthetic Joint Materials Evaluated on a Multichannel Screening Device. J Biomed Mater Res 16: 177-178, 1982.

149. Members Of Consensus Development Panel: Total hip joint replacement: Report of consensus development conference held by the nationalinstitutes of health. Orthop Rev 11: 129-138, 1982.

150. Michel R: Trace element burdening of human tissues due to the corrosion of hip-joint prostheses made of cobalt-chrome alloys. Arch Orthop Traum Surg 103: 85-95, 1984.

151. Michel R: Zum Uebertritt von Korrosions Produkten aus Stahl-Implantaten in das Kontaktgewebe. Z Orthop 118: 793-803, 1980.

152. Mittelmeier H: Selflocking ceramic total hip prosthesis. Orthop Trans 5: 376-382, 1981.

153. Miyanaga Y, Kurosawa H: Contact study of the hip joint. Arch Orthop Traum Surg I03: 13-17, 1984.

154. Mjoberg B, Pettersson H, Rosenqvist R, Rydholm A; Bone Cement, Thermal Injury and the Radiolucent Zone. Acta Orthop Scand 55: 597-600, 1984.

155. Moore HF. Fatigue tests, in: Metals Handbook. Ed. American Society for Metals, 118-124, Philadelphia, 1948. 
156. Morancho R, Ghommidh J, Constant G, Moyen B, Comtet JJ, Santini R, Buttazzoni B: New hydroxyapatite coatings obtained by chemical spray process and their biological behaviour. In: Ceramics in Surgery, pp. 97-104, Ed. Vincenzini, Elsevier, Amsterdam, 1983.

157. Morscher EW, Dick W: Cementless fixation of "isoelastic" hip endoprostheses manufactured from plastic materials. Clin Orthop 176: 77-87, 1983.

158. Ogino M, Ohuchi F, Hench LL: Compositional dependance of the formation of calciumphosphate films on bioglass. J Biomed Mater Res 14:55-64, 1980.

159. Oh I, Merckx DB, Harris WH: Acetabular Cement Compactor: An Experimental Study of Pressurization of Cement in the Acetabulum in Total Hip Arthroplasty. Clin Orthop 177: 289-293, 1983.

160. Oh I: A Comprehensive Analysis of the Factors Affecting Acetabularcup Fixation and Design in Total Hip Replacement Arthroplasty: A Series of Experimental and Clinical Studies. Hip Proc Hip Soc 129-177, 1983.

161. Oh J, Harris WH: Proximal strain distribution in the loaded femur. J bone Joint Surg 60A: $75-85,1978$.

162. Olmstead ML, Hohn RB, Turner TM: A five-year study of 221 total hip replacements in the dog. J Am Veter Med Ass 183: 191-194, 1983.

163. Olmstead ML, Hohn RB, Turner TM: Technique for canine total hip replacement. Veter Surg 10: 44-50, 1981.

164. Osborn JF, Heise U, Böker KD: Neue Konzepte der Osteoplastik durch die Einbeziehung von Hydroxyl-apatit Keramik in Kieferchirurgie, Orthopaedie und Neurochirurgie. In: Die Aesthetik von Form Und Funktion in der Wiederherstellungschirurgie, Ed. G Pfeifer, Springer, Heidelberg, 1985.

165. Osborn JF: Biowerkstoffe und Ihre Anwendung bei Implantaten. Schw Mschr Zahnheilk 89: 1138-1139, 1979.

166. Osborn JF: Personal communications. 1987.

167. Osborn JF: The biological behaviour of the hydroxyl-apatite ceramic coating on a titanium stem of a hip prosthesis - The first histological evaluation of human autopsy material. Biomed Technik 32: 177-183, 1987.

168. Panush RS, Petty RW: Inhibition of human lymphocyte responses by methylmethacrylate. Clin Orthop 134: 356-363, 1978.

169. Patka P, Den Hollander W, Den Otter G, Heidendal GAK, De Groot K: Scintigraphic Studies to Evaluate Stability of Ceramics (hydroxyapatite) in Bone Replacement. J Nucl Med 26: 263-271, 1985.

170. Patka P, Otter G den, Groot K de: Vervangen van botweefsel. Ned Tijdschr Geneeskd 129: 1416-1421, 1985.

171. Patka P: Bone replacement by calcium-phosphate ceramics. Thesis, Free University, Amsterdam, 1984.

172. Paul HA, Bargar WL: Histologic changes in the dog acetabulum following total hip replacement with current cementing techniques. J Arthroplasty 2: 71-76, 1987.

173. Pedersen JG, Lund B, Reimann I: Depressive effects of acrylic cement components on bone metabolism. Isotope release and phosphatase production studied in vitro. Acta Orthop Scand 54: 796-801, 1983. 
174. Peelen JGJ, Rejda BV, Groot K de: Preparation and properties of sintered hydroxyl-apatite. Ceramurgia 4: 71-74, 1978.

175. Perren SM: The induction of bone resorption by prosthetic loosening. In: Morscher E, Cementless fixation of hip prostheses: 39-41, Springer, New York, 1983.

176. Petty W: The effect of methylmethacrylate on chemotaxis of polymorphonuclear leucocytes. J Bone Joint Surg 60A: 492-498, 1978.

177. Petty W: The effect of methylmethacrylate on bacterial phagocytosis and killing by human polymorphonuclear leucocytes. J Bone Joint Surg 60A: 752-757, 1978.

178. Pilliar RM, Cameron HU, Binnington AG, Szivek J, Macnab I: Bone Ingrowth and Stress Shielding with a Porous Surface Coated Fracturefixation Plate. J Biomed Mater Res 13: 799$810,1979$.

179. Pilliar RM: Powder Metal-made Orthopaedic Implants with Porous Surface for Fixation by Tissue Ingrowth. Clin Orthop Rel Res 176: 42-51, 1983.

180. Ponssen P, Groot K de: Medical use of calcium-phosphate ceramics. In Bioceramics of calcium-phosphate, Ed.K de Groot, CRC Press, Boca Raton, 1983.

181. Putter C de, Groot K de, Sillevis Smit PAE: Permucosal implants of dense hydroxyl-apatite in dogs. J Prosth Dent 49: 87-95, 1983.

182. Putter C de: Permucosal Dental Implants of Dense hydroxyl-apatite. Thesis, Free University, Amsterdam, 1984.

183. Rae T: The toxicity of metals in orthopaedic prostheses. J Bone Joint Surg 63B: 435-440, 1981.

184. Ranawat CS, Johanson NA, Rimnac CM, Wright TM, Schwartz RE: Retrieval Analysis of Porous-coated Components for Total Kneearthroplasty: A Report of two Cases. Clin Orthop 209: 244-248, 1986.

185. Reckling FW: The bone cement interface temperature during total joint replacement. J Bone Joint Surg 59A: 80-82, 1977.

186. Reikeras O: Mechanical effects of reaming and implantation of acrylic cement into the medullary cavity of bones. Arch Orthop Traum Surg 103: 120-124, 1984.

187. Rejda BV: Composite materials for hard tissue replacement. Thesis, Free University, Amsterdam, 1977.

188. Renooij W, Hoogendoorn HA, Visser WJ, Lentferink RHF, Schmitz MGJ, Vanieperen H, Oldenburg SJ, Janssen WM, Akkermans LMA, Wittebol P: Bioresorption of Ceramic Strontium-85-labeled Calcium Phosphate Implants in Dog Femora: A Pilot Study to Quantitate Bioresorption of Ceramic Implants of Hydroxyapatite and Tricalcium orthophosphate in vivo. Clin Orthop 197: 272-285, 1985.

189. Rens ThJG van: Totale Heupprothese, Indikaties, Resultaten, Levensduur. Ned Tijdschr v Geneeskd 130: 1782-1787, 1986.

190. Ring PA: Five to fourteen year interim results of uncemented total hip arthroplasty. Clin Orthop 137: 87-95, 1978.

191. Ritter JE: Engineering design and failure of brittle materials. In Fracture Mechanics of Ceramics (Brad RC ed) Plenum Press NY, 667-680, 1974.

192. Ritter MA, Meding JB: A comparison of osteonecrosis and osteoarthritis patients following total hip arthroplasty: a long-term follow-up study. Clin Orthop 206: 139-146, 1986. 
193. Ritter MA: Correlation of prosthetic femoral head size and design with longevity of total hip arthroplasty. Clin Orthop 176: 252-257, 1983.

194. Ronningen H, Solheim LF, Langeland N: Invasion of Bone into Porous Fiber Metal Implants in Cats. Acta Orthop Scand 55: 352-358, 1984.

195. Ronningen H, Solheim LF, Langeland N: Bone Formation Enhanced by Induction, Bone Growth in Titanium Implants in Rats. Acta Orthop Scand 56: 67-71, 1985.

196. Rose RM, Crugnola A: On the origins of high in vivo wear rates in poly-ethylene components of total joint prostheses. Clin Orthop 145: 277-286, 1979.

197. Rose RM, Radin EL: True wear rate of UHMW poly-ethylene in total hip prostheses. J Bone Joint Surg 62A: 537-549, 1980.

198. Rosenquist R, Bylander B, Knutson K, Rydholm U, Rooser B, Egund N, Lidgren L: Loosening Of the porous Coating Of bicompartmentalprostheses in Patients with Rheumatoid Arthritis. J Bone Joint Surg (Am) 68-A: 538-542, 1986.

199. Roth H, Muller W: Zur Behandlung grossvolumiger Knochendefekte im Kieferbereich mit Hydroxyl-apatit Granulat. Schweiz Mschr Zahnmed 94: 222-225, 1984.

200. Rubin CT, Lanyon LE: Regulation of bone formation by applied dynamic loads. J Bone Joint Surg 66A: 397-402, 1984.

201. Ruegsegger P, Seitz P, Gschwend N, Dubs L: Disuse osteoporosis in patients with total hip prostheses. Arch Orthop Trauma Surg 105: 268-273, 1986.

202. Ryu RKN, Bovill EG, Skinner HB, Murray WR: Soft Tissue Sarcomaassociated with Aluminum Oxide Ceramic Total Hip Arthroplasty: A Casereport. Clin Orthop 216: 207-212, 1987.

203. Sela J, Gross U: Primary mineralization and extra-cellular matrix vesicles in rat bone after administration of glass-ceramic implants. Arch Orthop Traum Surg 98: 236-240, 1981.

204. Saha S: Longitudinal shear properties of human compact bone. J Mater Science 12: 1798, 1977.

205. Salvati EA, Wilson PD JR, Jolley MN, Vakili F, Aglietti P, Brown G: A Ten Year Followup of our First One-hundred Consecutive Charnley Total Hip Replacements. J Bone Joint Surg 63A: 753-767, 1981.

206. Scharbach H: A metal-ceramic composite design for cementless fixation to bone. In Morscher: Uncemented fixation of hip prostheses. Springer, Heidelberg, 1983.

207. Seidelmann U, Richter H, Soltesz U: Failure of Ceramic Hip-endoprostheses by Slow Crack Growth Lifetime Prediction. J Biomedmater Res 16: 705-714, 1982.

208. Semlitsch $\mathbf{M}$ : Metallic implant materials for hip joint endoprostheses designed for cemented and cementless fixation. In Ed. Morscher: Cementless fixation of hip implants. Springer, Heidelberg, 1983.

209. Shimazaki K, Mooney V: Comparative Study of Porous Hydroxyapatite and Tricalcium Phosphate as Bone Substitute. J Orthop Res 3: 301-310, 1985.

210. Tapadiya D, Walker RH: Prediction of outcome of total hip arthroplasty based on initial postoperative radiographic analysis. Clin Orthop 186: 5-15, 1984.

211. Taysum DA, Evans FG, Hammer WH, Jee WSS, Rehfeld CE, Blake LW: Radionuclide and bone strength. In: Aspects of Internal radiation, p. 145, Pergamon Press, Oxford, 1962. 
212. Tracy BM, Doremus RH: Direct Electron Microscopy Studies of the Bone-Hydroxylapatite Interface. J Biomed Mater Res 18: 719-726, 1984.

213. Turner TM, Sumner DR, Urban RM, Rivero DP, Galante JO: A Comparative Study of Porous Coatings in a Weight-bearing Total Hip Arthroplasty Model. J Bone Joint Surg 68-A: 1396-1409, 1986.

214. Uchida A: The use of ceramics for bone replacement, a comparative study of three different porous ceramics. J Bone Joint Surg 66B: 269-275, 1984.

215. Volz RG, Greenwald AS: Biological ingrowth, fact and fiction. Report committee on biomedical engineering, Am Acad Orthop Surg, 1986.

216. Wainwright SA, Biggs WD, Curry JD, Gosline JM: Mechanical design in organisms. Wiley, New York, 1976.

217. Walker PS, Onchi K, Kurosawa H, Rodger RF: Approaches to the interface problem in total joint arthroplasty. Clin Orthop 182: 99-108, 1984.

218. Wilde AH, Greenwald AS: Shear strength of self-curing acrylic cement. Clin Orthop 106: 126-130, 1975.

219. Winter M, Griss P, Groot K de: Comparative Histo-compatibility Testing of Seven Calcium Phosphate Ceramics. Biomaterials 2: 159-163, 1981.

220. With G de, Dijk HJA van, Hattu N, Prijs K: Preparation, microstructure and mechanical properties of dense polycrystalline hydroxyl-apatite. J Biomed Mater Res 15: 527-535, 1981.

221. Wolff J: Das Gesetz der Transformation der Knochen, Berlin, 1892.

222. Wroblewski BM: 15-21-Year results of the Charnley low-friction arthroplasty. Clin Orthop 211: 30-35, 1986.

223. X-ray powder data file. American Society for Materials and Testing: cardnrs. 9-432, 9-348, 9-169; 1973.

224. Young RA: Biological Apatite vs Hydroxyapatite at the Atomic Level. Clin Orthop 113: 2249-2262, 1975.

225. Yue S, Pilliar RM, Weatherly GC: The Fatigue Strength of Porous-Coated Ti-6\%Al-4\%V Implant Alloy. J Biomed Mater Res 18: 1043-1058, 1984.

226. Zel JM van der, Groot K de: Patent "prestressed implants" Dutch number 81.01674, 1981.

227. Zweymuller K: A cementless titanium hip endoprosthesis system based on press-fit fixation: basic research and clinical results. Instr Course Lect 35: 203-225, 1986. 
Appendix

Data collection forms of european clinical multicenter hip study 
Clinical Data Coordinator

Dept. of Orthopaedic Surgery Maastricht University Hospital PO 1918

$6201 \mathrm{BX}$ Maastricht.

The Netherlands
Pre-OP patient

Medical History
Page 1 of 1

$\begin{array}{lr} & \text { Country } \\ \square I & \square F \\ \square E & \square B \\ \square G B & \square \mathrm{NL} \\ \square D & \square\end{array}$

No $01 / \ldots 1 \ldots \ldots 1$
Please all information in ink.

PATIENTS NAME: (Last. First)

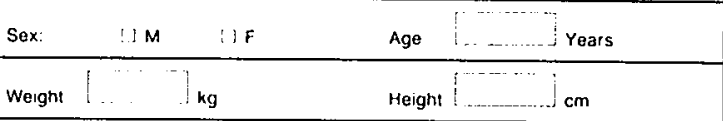

Hip indicated tor surgery (one form per hip)

[] right [i] lelt

\section{GENERAL HEALTH}

A. Normal. unilateral hip affected

B. Normal, bilateral hips affecied

C. Other, specily

$$
\text { Uni or bilateral }
$$

\section{PRIOR TREATMENT}

CHECK ALL THAT PERTAIN AND SPECIFY DATE

\section{Ii None}

i. A. Osteomy

i 1 B. Fracture treatment

(1) Intramedullary

(]) Blade/Plate

(1) Conservative

[i] Other

L.] C. Resection Arthroplasty

(1.) D. Surface Replacement

(.) E. Herni-Arthroplasty specily type

1.) Cemented

i.) Cementless

i] F. Total Hip specily type

i) Cemented

[] Cementless

G. Arthrodesis

I H. Treatment for infection of Hip. Prior organisme

:1t. Other. specity

COMMENTS

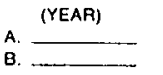

\section{INFLAMMATORY COLLAGEN}

TRAUMA PRIMARY HIP

\section{DEGENERATIVE}

ARTHRITIS

NECROSIS

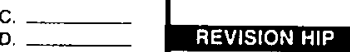

D.

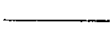

G.

H.

1
All items must be completed

RY DIAGNOSIS (And Secondary " Applicable)

Primary

A. [.] Osteoarthritis

B. 1] Congenitally Dysplastic Hip

C. Dost-Traumatic Arthritis

D. [. Legg-Perthes Disease

E. C) Slipped Capital Femoral Epiphysis

F. T) Post-Septic Arthritis

1. I] Other (specify)

G. ?] Idiopathic Avascular Necrosis

$H$, I] Steroid-Related Avascular Necrosis

I. Alcohol-Related Avascular Necrosis

J. Post-Traumatic Avascular Necrosis

2. [i] Other (specify)

K. [] Rheumatoid Armritis

L. J Juvenile Rheumatoid Arthritis

3. T.) Other (specify)

M. I.) Fresh-Femoral Neck Fractures

N. [: Acetabular Fracture

O. I Pseudo-Arthrosis Femoral Neck

P. I) Failure Osteosynthesis

4. I.] Other (specily)

O. I. Surface Replacement

R. Unipolar Heni Prosthesis

S. Bipolar Hemi Prosthesis

T. D Total Hip Replacement

5. [) Other (specify)

Secondary

[.] $\mathrm{A}$

[.) $\mathrm{B}$

i.: $\mathbf{C}$

[D.

[.] $\mathbf{E}$.

[i.) $F$.

[.] 1 .

[.) $\mathrm{G}$.

[] $\mathrm{H}$

[.] $\mathrm{I}$.

[] $\mathrm{J}$.

[.. 2.

[] $\mathrm{K}$.

it

L

3

D. $\mathrm{M}$.

I.: N.

..

P.

i. 4.

Lo.

R.

D. $\mathrm{s}$.

S.

i] 5 .

\section{AEASONS FOR REVISION (Check all that pertain)}

A. Femoral Component Loosening

B. A Acetabular Component Loosening

C. Component Breakage

C) Femoral Acelabular

D. Component Misalignment

C. Femorat $\square$ Acetabular

E. Inadequate Range of Motion

F. C] Heterotopic Calcification

G. $C$ Luxation

H. Pain

I. Intection

J. $\because$ Bone Fracture (locatron specify)

K. :-: Other (specify)

DATA COORDINATOR

DATE: (Year/Month/Oay)

INVESTIGATORS SIGNATURE

HOSPITAL

CITY

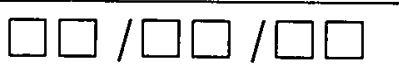


Patient Evaluation Record

$\begin{array}{lr}\square I & \text { Country } \\ \square E & \square B \\ \square G B & \square \mathrm{NL} \\ \square D & \square \ldots .\end{array}$

No $01 / \ldots \ldots 1 \ldots$

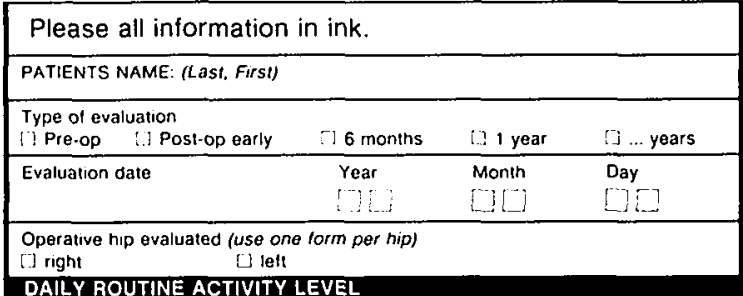

Activity level

[.] A. High

[i] B. Intermediate

[.] C. Low

\section{CURRENT MEDICATION}

(.) A. None

[.] B. Analgesic

(]) C. Other (specily)

\section{OTHER PROBLEM JOINTS}

(1) No

Ii Yes

Operative side

(.) A. Knee

i.] B. Ankle

[?] C. Foot

Non-operative side

'] A. Knee

[]] B. Ankle

C.) Coot

[.] D. Hip

\section{COMPLICATIONS}

Any post-operative complications develop?

[.] No

[]] Yes

If yes, report on Complications Form no. 4

\section{HIP PAIN}

(Check One For Operative Hip)

A. [.] No Pain

B. [.] Slight: Occasional Ache. No Compromise of Activities

C. Mild: No Eflect on Average Activities. May Take Aspirin

D. Moderate: Pain Tolerable. May Limit Activities, Occasıonal Medication

E. [? Marked: Severe Pain But Ambulatory. Activities Limited. Frequent Medication

F. I Disabled: Crippled. Pain in Bed. Bedridden

\section{ETHIOLOGY OF HIP PAIN}

INVESTIGATORS SUSPECTED ETIOLOGY

(Check All That Pertain And Give Explanation)

Primary Hip Disease
Acetabulum
$\vdots$ Stem Fixation
Bursitis
Synovitis
Wound Intection

[] Intermediate
[] Intermediate
[.] Intermediate
[.] Intermediate
$\square$ Intermediate
$\square$ Intermediate
$\square$ Intermediate

All items must be completed ACTIVE RANGE OF MOTION

(Specify Degrees Or Motions For Each Hip)

MOTION:

A. Flexion

B. Extension

C. Abduction

D. Adduction

E. Internal Rotation (in extension)

F. External Rotation (in extension)

OPERATIVE HIP: NON-OPERATIVE HIP:

\section{DEFORMITY}

(Check And Specity All That Pertain To Operative Hip) $\square$ None
A. $\square$ Fixed Adduction
B. Fixed Internal Rotation
C. $\square$ Fiex External Rotation
D. $\square$ Flexion Contracture
E. $\square$ Leg Length Discrepancy
Specify:
Specify:
Specity:
Specify:
Specify:
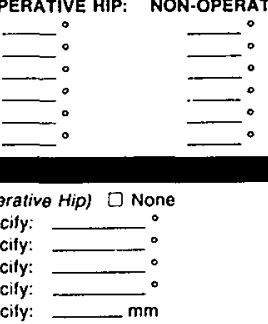

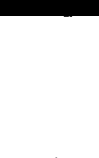

\section{STAIRS: (Check One)}

A. D Foot Over Foot Without Use of Banister

B. Fool Over Foot Using Banister

C. One Foot at a Time Without Use of Banister

D. C) One Foot at a Time Using Banister

E. U Using Stairs With Exter, tal Support and Banıster

F. $]$ Unable to Climb Stairs

SUPPORT: (Check One)

A. D No External Support Needed to Walk Comfortably

B. S Single Cane Needed For Long Walks

C. $\square$ Single Cane Needed Most of The Time

D. One Crutch Needed Most of The Time

E. Two Crutches or Walker Needed Most of The Time

F. $\square$ Unable to Walk

WALKING DISTANCE

A. $\square$ Unlimited Range

B. $\square(800-1500$ meters $)$

C. $\square$ (150- 800 meters)

D. Indoors, Around House Only

E. Bed to Chair

F. U Unable to Walk

\section{LIMP (Check One)}

F. $\square$ No Limp

A. $\square$ Mild Limp

B. $\square$ Moderate Limp

C. I Severe Limp

D. Cannot Walk Without Support

E. Cannot Walk

\section{SHOES AND SOCKS}

A. $\bigcirc$ Able

8. $\rightarrow$ Difficult

c. DUnable

\section{COMMENTS:}

Explanation:

\begin{tabular}{|l|l|}
\hline INVESTIGATOR & HOSPITAL CITY \\
\hline INVESTIGATORS SIGNATURE
\end{tabular}

INVESTIGATORS SIGNATURE
DATA COORDINATOR

Next scheduled vis!

$\square \square$ Year $\square$ Month $\square$

Day

DATE: (Year/Month/Day) 
Clinical Data Coordinator

Dept. of Orthopaedic Surgery Maastricht University Hospital PO 1918

6201 BX Maastricht,

The Netherlands

\section{Surgical details}

$\begin{array}{lr} & \text { Country } \\ \square I & \square F \\ \square E & \square B \\ \square G B & \square \mathrm{NL} \\ \square D & \square \ldots\end{array}$

No $01 / \ldots \ldots /$...

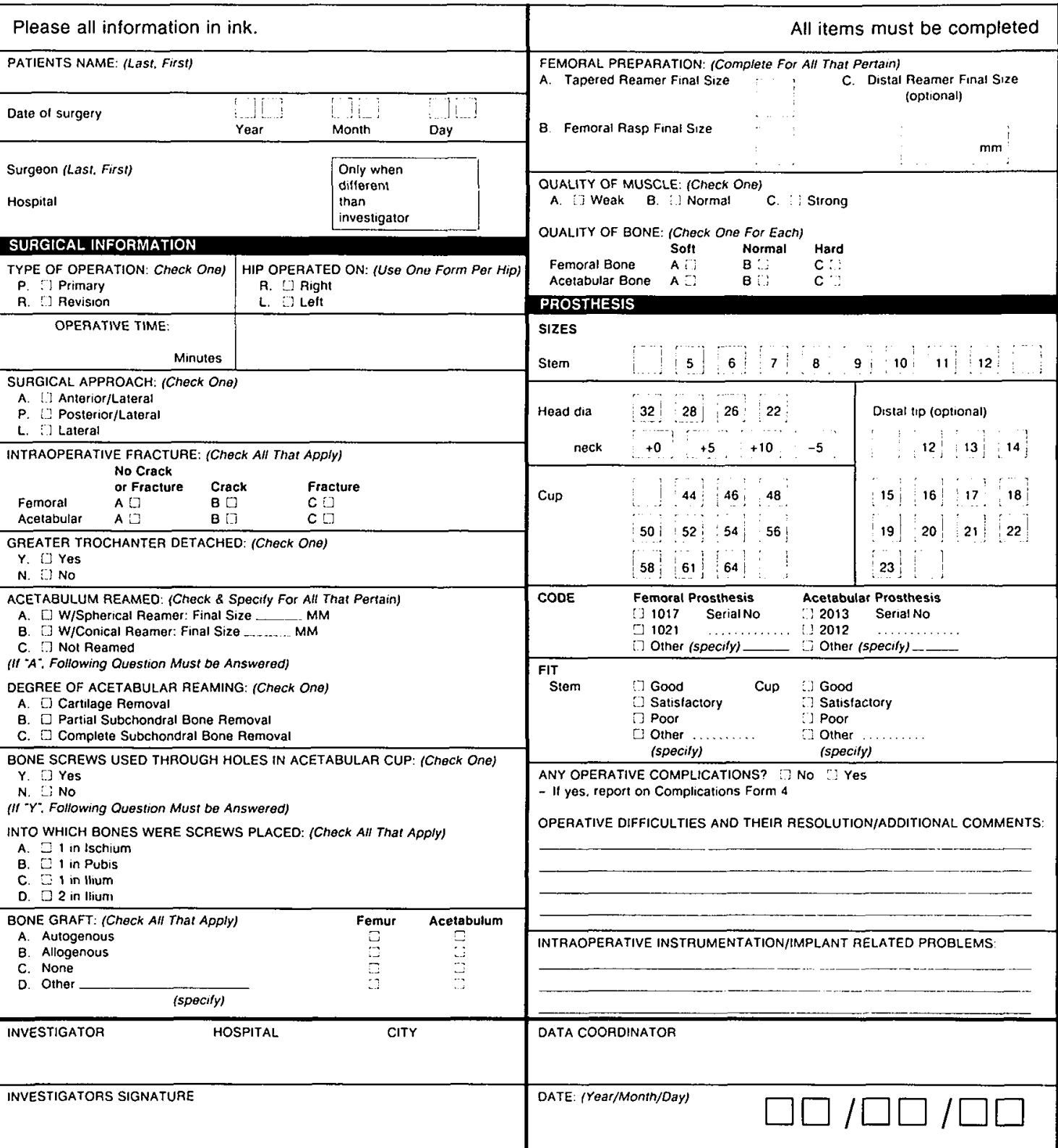


Clinical Data Coordinator Dept. of Orthopaedic Surgery Maastricht Unjversity Hospital PO 1918

6201 BX Maastricht.

The Netherlands
Complications ADVERSE EFFECTS

$\begin{array}{lr} & \text { Country } \\ \square I & \square F \\ \square E & \square B \\ \square G B & \square \mathrm{NL} \\ \square D & \square \ldots . .\end{array}$

No $01 / \ldots 1 \ldots \ldots$

Please all information in ink.

All items must be completed

PATIENTS NAME; (Last, First)

DATE COMPLICATION OBSERVED OR REPORTED:

OPERATIVE HIP; (One Form Per Hip) (Month, Oay, Year) $\square \square / \square \square / \square \square$

[i) Right : Lett

\section{COMPLICATIONS}

CHECK ALL THAT PERTAIN:

A. I Superficial Wound Infection

B. Deep Joint Intection

C. $\square$ Trochanteric Fracture

D. [] Femoral Fracture: 1. $\square$ intra-Operative 2. Tost-Operative

E. [] Dislocation

F $\square$ Loosening Femoral Component

G. D Loosening Acetabular Component

H. I) Subluxation

I. D Urinary Tract infection

J. D Pulmonary Embolism

K. IThrombophlebitis - Thrombus

L. $[$ Congestive Heart Failure

M. L Nerve Palsy

N. $\square$ Respiratory Infection

o. $\square$ Hip Pain

O. C) Perforation of Cortex

R. Ectopic/Heterotopic Bone

5. $\square$ Trochanteric Non-Union

P. $\square$ Other (Specity)
A. Revision - Acetabular Component

B. $\square$ Revision - Femoral Component

C. $\square$ Incision and Drainge

D. $D$ Closed Reduction

E. 1. D Open Reduction

F 2. Medication

H. Removal of Implant(s). But Not Replaced

G. D Other (Specify)

CIRCUMSTANCES SURROUNDING ONSET OF COMPLICATIONS

\section{DATE OF ONSET:}

(Month, Day, Year)

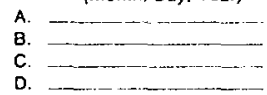

D.

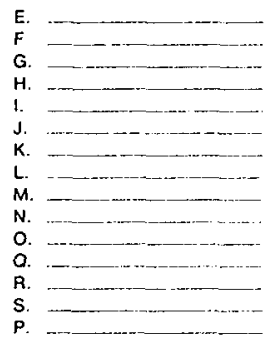

POSSIBLY RELATED TO PROSTHESIS?

$\begin{array}{lll}\text { A. } & \text { Nes } \\ \text { B. } & \text { YYes } & \text { No } \\ \text { C. } & \text { Y Y Yes } & \text { No }\end{array}$

E. :-: Y Y No

F I'Y Yes Y No

G. $\quad$ Yes $\quad i N \mathrm{No}$

H. IYes in No

I. IYes îno

J. $\mathrm{H}$ Yes

K. $\because$ Yes

M. Yes

N. YYes IINO

o. iyes : No

o. IY Yes ino

i) Yes

S. I] Yes lij

P. il Yes if No

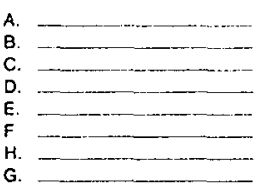

i) Satisfactory

Catisfactory

[1] Satisiactory

[] Satisfactory

4 Satisfactory

[.] Satisfactory

i.) Satislactory

G Satisfactory

1) Unsatisfactory

I Unsatisfactory

i) Unsatistaclory

i) Unsatisfactory

(i) Unsatislactory

[] Unsatisfactory

[] Unsatisfactory

() Unsatisfactory

COMMENTS:

\begin{tabular}{|l|l|l}
\hline INVESTIGATOR HOSPITAL & CITY & DATA COORDINATOR
\end{tabular}

INVESTIGATORS SIGNATURE

DATE: (Year/Month/Day) 


\section{OSTEONICS}

Clinical Data Coordinator

Dept. of Orthopaedic Surgery

Maasiricht University Hospital

PO 1918

6201 BX Maastricht.

The Netherlands
HYDROXYLAPATITE

HIP STUDY

Form 5

Page 1 of 1

\section{Visual X-ray}

Analysis

\section{Country}

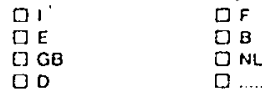

No $01 / \ldots-1$

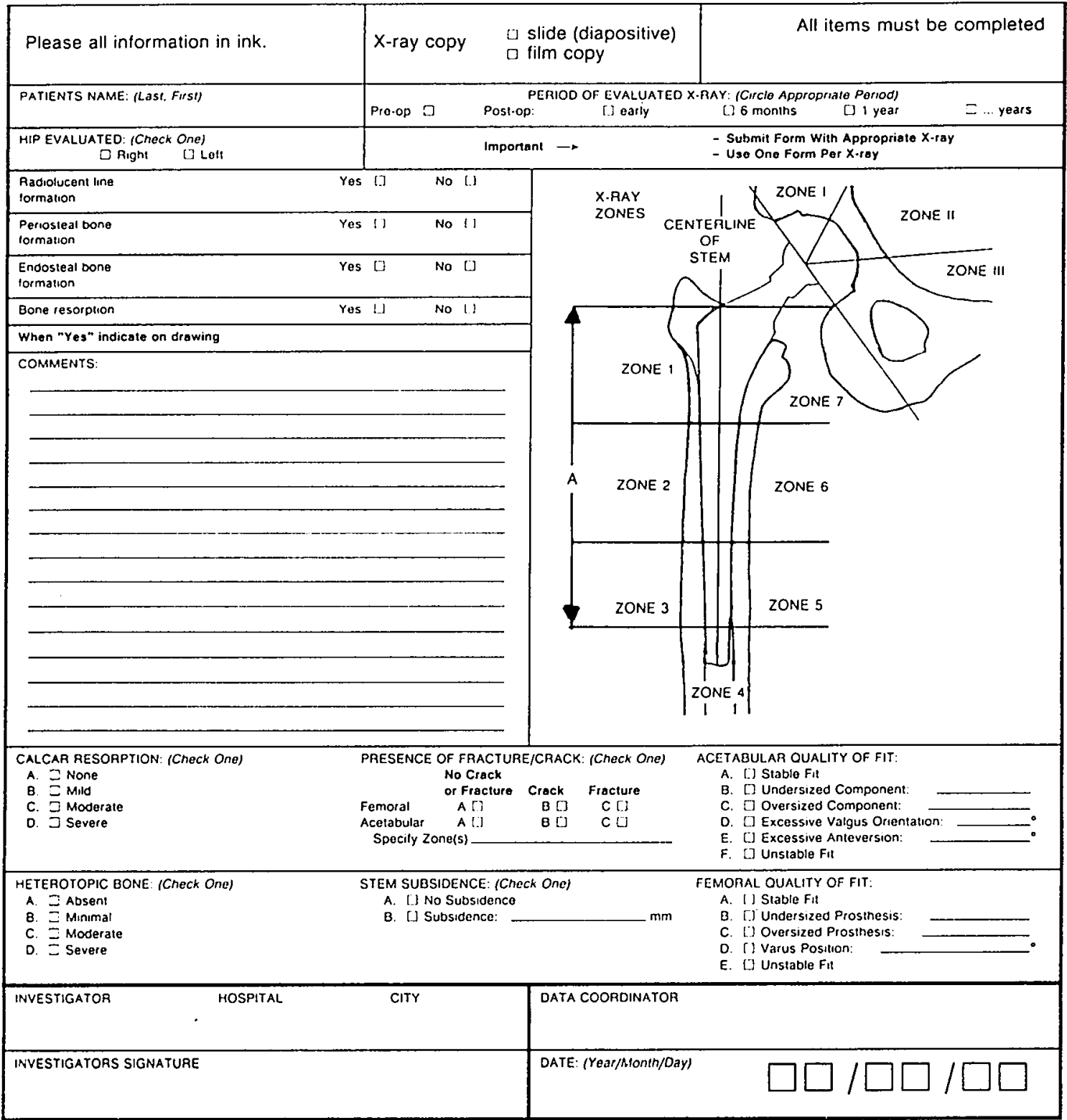


Clinical Data Coordinator

Dept. of Orthopdedic Surgery

Maastricht University Hospila

1 Revision/Removal

2 Patient Withdrawal

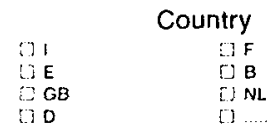

No $01 / \ldots . .1$

All items must be completed

Please all information in ink.

PATIENTS NAME: (Last. FirSt)

\section{REVISION / REMOVAL}

Date of revision

Year/Month/Day

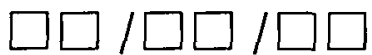

ij revision

inght hip

I removal

? lett hip

A. Radiological findings prior to removal or revision procedure

[.] Intormation on form 5 . sent with $X$.ray

i : No $X$-ray available. comment:

B. Brief sommary of cirsumstances leading to removal or revision procedure

\section{RETRIEVED HYOROXYLAPATITE COMPONENT INFOAMATION}

A. Note any difficulties encountered in removing Hydroxylapatite Component(s)

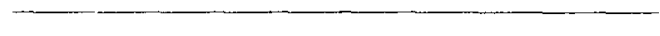

Femoral Shatt Split:

[.] No

Yes 1. Nondisplaced 2.:. Displaced

C. Pelvic Fractures

i. No

[1. Yes 1. 1.] Nondisplaced 2. Displaced

D. Ingrowth observed on retrieved Hydroxylapatite component(s)

(indicate location of ingrowth on component illustration)

Histological intormation available

[] Yes

No

II yes. please describe or/and enclose microscopies
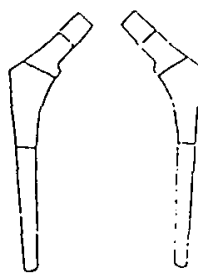

Femoral Component

\begin{tabular}{|c|c|}
\hline Amount. & F Type of Ingrown \\
\hline [.] Minuma! & 1 :... Bony \\
\hline : Moderate & 2 fibrous \\
\hline 3.. Substantıl & $3:$ Bom \\
\hline
\end{tabular}

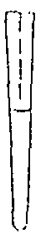

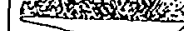

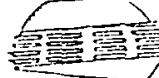

Acetabular Component

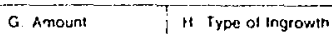

Mintmai

2: Moderate

3. Substantia

\section{WITHDRAWAL}

[1] Withdraw Irom investigation

i.) Moved to elsewhere

D. Died as a result ol causes unrelated to device

17 Other

Commen
- Reason:

- When known. please indicate

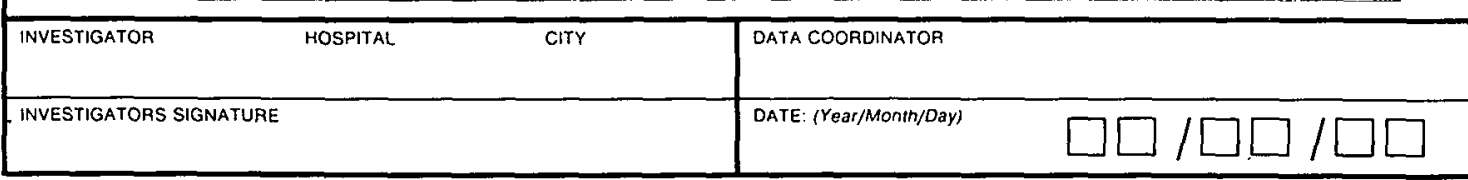




\section{Dankwoord}

Bij de voltooïng van dit proefschrift wil ik iedereen danken, die heeft bijgedragen aan de totstandkoming van dit werk.

Beste Klaas de Groot, de eerste ideeën omtrent mogelijke toepassingen van hydroxylapatiet in de orthopaedie zijn het resultaat van jouw gunstige ervaringen met dit materiaal in de tandheelkunde. Door veelvuldige kennis uitwisseling is het mogelijk gebleken het concept van biologische implantaatfixatie met apatiet-coatings over te dragen naar het vakgebied van de orthopaedie. Zonder jouw enthousiaste steun en kennis was dit proefschrift niet mogelijk geweest.

Beste Ton van der Linden, onder jouw hoede heb ik mij mogen bekwamen in de orthopaedie. Hartelijk dank voor de unieke wijze waarop jij altijd het goede voorbeeld hebt gegeven. Jouw langdurige ervaring en jouw gevoel voor relativeren hebben er veel toe bijgedragen om dit proefschrift in het juiste kader te plaatsen.

De referenten van dit proefschrift, prof dr G Kootstra, prof dr ir R Huiskes, prof dr HKL Nielsen, prof dr F Bosman, prof dr $\mathrm{S}$ van der Linden dank ik voor hun commentaar. In het byzonder dank ik Rik Huiskes voor zijn waardevolle kritische opmerkingen ten aanzien van de biomechanica van het heupgewricht.

Beste Christel Klein, jouw kennis van de biologie van calcium-phosphaat-keramische materialen heeft veel bijgedragen aan mijn ontwikkeling op dit gebied. In het byzonder dank ik jou voor jouw enthousiaste hulp bij het verwerken en mede beoordelen van de mikroskopische preparaten.

Joop Wolke en Hans van der Lubbe van het materiaalkundig laboratorium van de Vrije Universiteit te Amsterdam, jullie hebben veel werk verzet bij het vervaardigen van de apatietcoatings, de uitvoering van de materiaalproeven en het verwerken van de histologische preparaten, hartelijk bedankt.

Beste Ton van de Boogaard, onder jouw hoede heb ik de dierproeven kunnen uitvoeren. Jouw kennis en ervaring hebben veel bijgedragen om dit op een zo verantwoord mogelijke wijze te kunnen doen.

Guido Heidendal, Jaap Teule, nucleair-geneeskundigen, evenals de laboranten van de afdeling nucleaire geneeskunde, dank ik voor de hulp bij de vervaardiging en beoordeling van de botscans bij dit onderzoek.

Ruud Kruger, hoofd van het laboratorium voor fysiologie, May Bosten evenals de andere dierverzorgers van het biomedisch centrum dank ik van harte voor de tijd en energie, die zij aan dit projekt besteed hebben.

Els Meers, hartelijk bedankt voor je inzet bij het opstarten van de eerste experimenten en je hulp als klinisch data coordinator bij de verzorging van patiënten gegevens.

Odette Panis, evenals de andere röntgenlaboranten, dank ik voor de hulp bij de vervaardiging van de röntgenfoto's.

Jo Houben, Annie Giebels, evenals alle andere medewerkers van de audio-visuele dienst dank ik voor de vlotte en accurate wijze waarop zij fotografisch vakwerk afleveren.

Chris Voskamp, medisch illustrator, dank ik voor de vervaardiging van de illustraties en de verzorging van de layout van dit proefschrift.

Martine Heidendal dank ik voor de franse vertaling van de samenvatting.

Paul Serekian, firma Osteonics, dank ik voor de taalkundige beoordeling van mijn 
engelstalige manuscript, evenals voor de vele kritische vaktechnische discussies, die de kwaliteit van het onderzoek wezenlijk hebben bevorderd.

Voorts dank ik direktie en medewerkers van de firma Osteonics, in het byzonder Alex Kowaylo, Bob Averill, Joe d'Errico, Alex Banjé en Gied Hermse voor de genereuze wijze waarop zij dit projekt zowel financieel als logistiek hebben ondersteund.

Mijn collega stafleden André van Ooy, Geert Walenkamp, Henk Arens, Jan Verhaar evenals alle assistenten, dank ik voor de goede samenwerking en opvang in de kliniek, waardoor de mogelijkheid gegeven werd dit proefschrift te voltooien.

Vader en moeder, jullie hebben je veel moeite gegeven mij deze opleiding te kunnen geven. Dit werk is ook aan jullie opgedragen.

Hettie, Ingrid, Margriet en Carolien, jullie hebben je echtgenoot of vader vaak moeten missen. Ik besef, dat de offers groot zijn geweest, maar ik hoop toch ook, dat de resultaten van dit werk ook op jullie zullen afstralen.

Ruud Geesink 


\section{Curriculum vitae}

1-8-47 Geboren te Arnhem

19-6-65 Eindexamen gymnasium- $\beta$, Christelijk Lyceum te Arnhem.

1-12-72 Artsexamen, Vrije Universiteit Amsterdam.

1973 Dienstplichtig militair arts bij de Koninklijke Luchtmacht.

1974-1976 Opleiding algemene heelkunde, Gemeente Ziekenhuis Dordrecht, opleider dr. J. Kweekel.

1976-1980 Opleiding orthopaedie, Ziekenhuis Annadal Maastricht, opleider prof.dr. A.J. van der Linden.

1980-heden Staflid afdeling orthopaedie, Academisch Ziekenhuis Maastricht in samenwerking met prof.dr. A.J. van der Linden, dr. A. van Ooy, dr. G.H.I.M. Walenkamp, H. Arens en J. Verhaar 